\title{
Cellular automata in operational probabilistic theories
}

\author{
Paolo Perinotti
}

QUIT Group, Dipartimento di Fisica, Università degli studi di Pavia, and INFN sezione di Pavia, via Bassi 6, 27100 Pavia, Italy

The theory of cellular automata in operational probabilistic theories is developed. We start introducing the composition of infinitely many elementary systems, and then use this notion to define update rules for such infinite composite systems. The notion of causal influence is introduced, and its relation with the usual property of signalling is discussed. We then introduce homogeneity, namely the property of an update rule to evolve every system in the same way, and prove that systems evolving by a homogeneous rule always correspond to vertices of a Cayley graph. Next, we define the notion of locality for update rules. Cellular automata are then defined as homogeneous and local update rules. Finally, we prove a general version of the wrapping lemma, that connects CA on different Cayley graphs sharing some small-scale structure of neighbourhoods.

\section{Contents}

1 Introduction

2 Detailed outlook

3 Operational Probabilistic Theories 4

3.1 Formal framework . . . . . . . 5

3.2 Causality and the no-restriction hypothesis ........... 8

3.3 Norms . . . . . . . . . . . 10

4 The quasi-local algebra in OPTs 13

4.1 Quasi-local effects . . . . . . . . . 14

4.2 Extended states . . . . . . . . . . . 19

4.3 Quasi-local transformations ... . 22

5 Global update rules $\quad 31$

5.1 Update rule . . . . . . . . . . . . 32

5.2 Admissibility and local action . . . 35

Paolo Perinotti: paolo.perinotti@unipv.it, http://www.qubit.it
5.3 Causal influence. . . . . . . . . 37

5.3.1 Relation with signalling . . 38

5.4 Block decomposition . . . . . . . 39

6 Homogeneity 41

$\begin{array}{lll}7 & \text { Locality } & 47\end{array}$

8 Cellular Automata $\quad 51$

8.1 Results............ 51

9 Examples $\quad 55$

9.1 Classical case . . . . . . . . . 55

9.2 Quantum case . . . . . . . . . 55

9.3 Fermionic case . . . . . . . . . 56

10 Conclusion $\quad 56$

$\begin{array}{ll}\text { Acknowledgments } & 57\end{array}$

A Identification of the sup- and operational norm for effects

B Quasi-local states $\quad 57$

C Proof of identity $94 \quad 64$

D Homogeneity and causal influence $\quad 64$

E Proof of right and left invertibility of a locally defined GUR

F Proof of theorem 10 65

G Proof of lemmas 66 and $67 \quad 66$

References

\section{Introduction}

In the last two decades a new approach to quantum foundations arose, grounded on the quantum information experience $[1,2,3,4]$. This line of research on the fundamental aspects of quantum theory benefits from new ideas, concepts and 
methods $[5,6,7]$ that lead to a wealth of remarkable results $[8,9,10,11]$. In particular, quantum theory can be now understood as a theory of information processing, that is selected among a universe of alternate theories [12, 13, 1, 14] by operational principles about the possibility or impossibility to perform specific information processing tasks $[9,15]$.

The scenario of alternate theories among which the principles select quantum theory is called the framework of Operational Probabilistic Theories (OPTs) [8, 15], and has connections with the less structured concept of Generalized Probabilistic Theory (GPT) [5, 16, 17], as well as the diagrammatic category theoretical approach often referred to as quantum picturialism $[18,19]$. Inspiration for the framework came also from quantum logic [20, 21].

Quantum theory, namely the theory of Hilbert spaces, density matrices, completely positive maps and POVMs (in particular we refer to the elegant exposition of Ref. [22]), can thus be reformulated as a special theory of information processing. Besides the many advantages of this result, one has to face a main issue: the theory as such is devoid of its physical content. Elementary systems are thought of as elementary information carriers - brutally speaking, memory cells - rather than elementary particles or fields in space-time. While this framework is satisfactory for an effective, empirical description of physical experiments, when it comes to provide a theoretical foundation for the physics of elementary systems, the informational approach at this stage calls for a way to re-embrace mechanical notions such as mass, energy, position, space-time, and complete the picture encompassing the dynamics of quantum systems.

A recent proposal for this endeavour is based on the idea that physical laws have to be ultimately understood as algorithms, that make systems evolve, changing their state, exactly as the memory cells of a computer are updated by the run of an algorithm [23, 24, 25]. Such a program already achieved successful results in the reconstruction of Weyl's, Dirac's and Maxwell's equations (for a comprehensive review see Ref. [26]). The most natural candidate algorithm for describing a physical law in this context is a cellular automaton. The theory of cellular automata is a wide and established branch of computer sci- ence. The notion of a cellular automaton for quantum systems was first devised as the quantum version of its classical counterpart, e.g. in Refs. [27, 28, 29, 30], but turned out to give rise to a rather independent theory, developed starting from Ref. [31]: the theory of Quantum Cellular Automata (QCAs). The latter counts presently various important results - see e.g. Refs. [32, 33], just to mention a few. We stress that, most commonly, QCAs are defined to be reversible algorithms, and most results in the literature are proved with this hypothesis. It is known, however, that many desirable preoperties fail to hold in the irreversible case (see e.g. Ref. [34]). In the present work we will not consider irreversible cellular automata, and leave this subject for further studies.

In order to use cellular automata as candidate physical laws in the foundational perspective based on OPTs, one has two choices at hand. The first one is to start treating automata within a definite theory, and this is the approach adopted so far, in particular within Fermionic theory [35, 36, 37]. In the linear case, Fermionic cellular automata reduce to Quantum Walks, and this brings in the picture all the tools from such widely studied topic $[38,39,40,41,42,43]$. The non-linear case is far less studied [44, 45], and does not offer as many results for the analysis. Needless to say, this approach faces difficulties that are specific of the theory at hand, and prevents a comparison of different theories on a ground that is genuinely physical.

The second approach is initiated in the present paper, and consists in defining cellular automata in the general context of OPTs. This perspective offers the possibility of extracting the essential features of the theory of quantum or Fermionic cellular automata, those that are not specific of the theory but are well suited in any theory of information processing. As a consequence, one can generalise some results, and figure out why and how others fail to extend to the broader scenario. Moving a much less structured mathematical context, this approach can use only few tools, but provides results that have the widest applicability range. 


\section{Detailed outlook}

In this section we provide a short, non technical discussion of the main results. The purpose of this work is to define cellular automata in OPTs, and prove some general results that will help applying the theory to special cases of interest.

A cellular automaton is an algorithm that updates the information stored in an array of memory cells in discrete steps, in such a way that one needs to read the content of a few neighbouring cells to determine the state of a given cell at the next step. Cellular automata in the literature are often, but not always, defined to be homogeneous: in this case the local rule for the update of the cell is the same for every cell. Here we will adopt homogeneity, but the subject is presented so that the generalisation of definitions to inhomogeneous CA is straightforward.

Typically, the interesting case of a cellular automaton is the one involving an infinite memory array. The first challenge we have to face is then to extend the theory of OPTs from finite, arbitrarily large composite systems to actually infinite ones. This piece of theory has an interest per se, for many reasons ranging from the possibility to introduce thermodynamic limits to the extension of the theory of $\mathrm{C}^{*}$ and von Neumann algebras.

We then start with a review of the framework of OPTs, and build the necessary tools to define infinite composite systems. The starting point is the construction of mathematical objects that describe measurements on finitely many systems within an infinite array - the OPT counterpart of the space of local effects of quantum theory. Effects for the infinite system are then defined as limits of Cauchy sequences of local effects. Since the introduction of a suitable topology for the definition of limits is needed, we open the paper with section 3, where a review of OPTs is provided, along with a few new results that will be useful, and a rather consistent part of the section will be dedicated to the introduction and discussion of norms that will provide the necessary topological framework for a consistent definition of limits.

The subject of the subsequent Section 4 is then the construction of the Banach space of effects for the infinite composite system, and consequently the construction of the space of states as suitable linear functionals on effects. An important subsection will be dedicated to the construction of the algebra of quasi-local transformations, that allows for the description of transformations on an infinite system that can be arbitrarily well approximated by local operations on finitely many subsystems. Indeed, an important part of the theory of CAs in OPTs is built by ruling the way in which quasi-local transformations are transformed by the CA. In particular, the way in which the CA propagates the effects of a local transformation on surrounding subsystems will be the key to the definition of the neighbourhood of the subsystem, a concept that is central to the theory of CAs.

Once this is done, the next step consists in defining update rules, and their admissibility conditions, which are the subject of Section 5, along with causal influence and a block-decomposition theorem. Update rules are defined in the first place as automorphisms $\mathscr{V}$ of the space of quasilocal effects, but an important request they must abide is that when they act on a quasi-local transformation $\mathscr{A}$ by conjugation as $\mathscr{V} \mathscr{A} \mathscr{V}^{-1}$, the obtained transformation is again quasi-local.

The next step is taken in section 6, and consists in defining the property of homogeneity. The latter presents with some difficulties, stemming from the fact that, as we mentioned above, we are defining update rules prior to any mechanical notion. This implies that even space-time is not available at this fundamental stage, and without geometry we cannot define homogeneity as translational invariance under the group corresponding to a given space-time. On the contrary, we will define homogeneity by formalising the idea that every single cell has to be treated equally by the update rule, and this notion will be defined operationally, requiring that no experiment made of local operations will allow establishing any difference between cells. As a consequence of homogeneity, one can prove that every homogeneous update rule is underpinned by the Cayley graph of some group, generalising a result of Refs. [25, 46]. The mathematical theory known as geometric group theory then tells us that the Cayley graph representing the causal connections of cells in the memory array, being a metric space, uniquely identifies an equivalence class of metric spaces, that captures both the algebraic and geometric essential features of the group. Astonishingly, for Cayley graphs of finitely presented groups - which is the case for cellular automata 
as we define them here - the equivalence class always contains a smooth manifold of dimension at most four (see Ref. [47], pag. 90). This result means that we can always think of a cellular automaton as if it was embedded in a Riemannian manifold, in such a way that the Riemannian distance between nodes is almost the same as the distance between nodes given in terms of steps along the graph edges.

Also the notion of locality, presented in section 7 , comes with its own difficulties, that can be overcome by proving a generalisation of the result known as "unitarity plus causality implies localizability" [32]. This is where the theory, which is inspired by that of QCAs, deeply differs from the theory of classical cellular automata. In particular, considering the collection of transformations $\mathscr{V} \mathscr{A} \mathscr{V}^{-1}$ for all $\mathscr{A}$ acting on a given system $g$, we will define the neighbourhood of $g$ as the set of systems on which transformations $\mathscr{V} \mathscr{A}^{-1}$ act non trivially.

Once the theory of cellular automata is fully developed, some results are proved in Section 8. In particular, a very useful theorem is the wrapping lemma, which under very wide hypothesesthough not universal - allows for the classification of automata on a given infinite graph by classifying automata on any suitably "wrapped" finite version of the same graph.

In Section 9, a few examples are reviewed. In particular, using the general notion of locality, we apply the definition of causal influence and the neighbourhood scheme to the case of classical cellular automata. With the above definitions at hand, we show that allegedly local automata are actually non-local. This solves a long standing puzzle about the connection between classical and quantum automata - the so-called quantisation of classical automata [31, 48].

The paper is concluded by Section 10 with a summary of the results and some closing remarks.

\section{Operational Probabilistic Theories}

In this section we will provide a thorough introduction to OPTs, which is partly a review of the literature, and partly presentation of new results that will be used in the remainder. We provide here a sketchy introduction before going through formal definitions, and use Quantum Theory as an illustrative example for the main notions in the framework.

Quantum theory is about system types A 1 -namely complex Hilbert spaces $\mathrm{H}_{\mathrm{A}}$ that are classified by their dimension $d_{\mathrm{A}}=\operatorname{dim}\left(\mathrm{H}_{\mathrm{A}}\right)$-and transformations that can occur on systems as a consequence of undergoing a test. Tests are represented by quantum instruments $\mathrm{E}_{\mathrm{X}}=\left\{\mathscr{E}_{i}\right\}_{i \in \mathrm{X}}$, i.e. a collection of Completely Positive (CP) maps $\mathscr{E}_{i}$ that sum to a trace-preserving one (a channel): $\mathscr{E}=\sum_{i \in \mathrm{X}} \mathscr{E}_{i}$. The quantum operation $\mathscr{E}_{i}-\mathrm{a} \mathrm{CP}$ trace non-increasing map - represents the change in the system occurring upon the event of a specific outcome $i \in \mathrm{X}$ in the test. In the diagrammatic language of OPTs systems are represented as labelled wires, and instruments or quantum operations as boxes with an input and output wire, e.g.

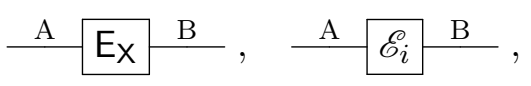

respecitvely. States can be considered as special transformations where the input system is I, having $\mathrm{H}_{\mathrm{I}}=\mathbb{C}$, i.e. $d_{\mathrm{I}}=1$. Notice that the a preparation test, i.e. the most general test from $\mathrm{I}$ to $\mathrm{A}$, represents a probabilistic preparation procedure where some state in the collection $\mathrm{P} \mathbf{X}=\left\{\rho_{i}\right\}_{i \in \mathrm{X}}$ is prepared, with the sub-normalised density matrix $\rho_{i}$ occurring upon reading the outcome $i \in \mathrm{X}$. The probability of occurrence of $\rho_{i}$ is $\operatorname{Tr}\left[\rho_{i}\right]$, and $\rho=\sum_{i \in \mathrm{X}} \rho_{i}$ has unit trace. Preparation tests or states for system A are represented by special diagrams

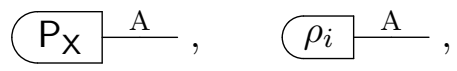

respectively. The space $\mathcal{H}_{\mathrm{A}}$ of Hermitian operators on $\mathrm{H}_{\mathrm{A}}$, whose dimension is $D_{\mathrm{A}}=d_{\mathrm{A}}^{2}$, is the real span of states of system $\mathrm{A}$.

A POVM $Q_{Y}=\left\{Q_{j}\right\}_{j \in \mathrm{Y}}$ is a collection of effects $0 \leq Q_{j} \leq I_{\mathrm{A}}$ that sum to the identity operator: $\sum_{j \in \mathrm{Y}} Q_{j}=I_{\mathrm{A}}$. This kind of test can be seen as a quantum instrument from A to I, namely a collection of linear functionals on the real space spanned by density matrices, where the functionals $a_{j}(\rho)$ are defined as $a_{j}(\rho):=\operatorname{Tr}\left[\rho Q_{j}\right]$. The

\footnotetext{
${ }^{1}$ We remark that for the purpose of information processing the type of a system is captured by the dimension of the corresponding Hilbert space, e.g. the electron spin is of the same type as the photon polarisation. This is slightly different than the usual notion of system type in physics, where the two types in the above example are different.
} 
diagrammatic representation of POVMs and effects is the following

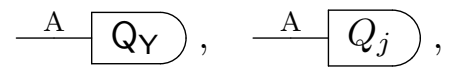

respectively.

Composite systems, such as $\mathrm{AB}$, correspond to to the Hilbert space $\mathrm{H}_{\mathrm{A}} \otimes \mathrm{H}_{\mathrm{B}}$ of dimension $d_{\mathrm{AB}}=$ $d_{\mathrm{A}} d_{\mathrm{B}}$. Tests $\left\{\mathscr{E}_{i}\right\}_{i \in \mathrm{X}}$ from A to B and $\left\{\mathscr{F}_{j}\right\}_{j \in \mathrm{Y}}$ from B to $\mathrm{C}$ can be run in sequence, obtaining a new test: $\left\{\mathscr{D}_{i j}\right\}_{(i, j) \in \mathrm{X} \times \mathrm{Y}}$ where $\mathscr{D}_{i j}:=\mathscr{F}_{j} \mathscr{E}_{i}$. On the other hand, tests $\left\{\mathscr{E}_{i}\right\}_{i \in \mathrm{X}}$ from A to B and $\left\{\mathscr{E}_{j}\right\}_{j \in \mathrm{Y}}$ from B to $\mathrm{B}^{\prime}$ on subsystems of a composite system $\mathrm{AA}^{\prime}$ can be run in parallel, obtaining the test $\left\{\mathscr{D}_{i j}\right\}_{(i, j) \in \mathrm{X} \times \mathrm{Y}}$ with $\mathscr{D}_{i j}:=\mathscr{E}_{i} \otimes \mathscr{F}_{j}$. In diagrams, we draw for quantum operations

$$
\begin{aligned}
& \mathrm{A} \mathscr{F}_{j} \mathscr{E}_{i} \mathrm{C}=\mathrm{A} \mathscr{E}_{i} \mathrm{~B} \mathscr{F}_{j} \mathrm{C}, \\
& \mathrm{AA}^{\prime} \mathscr{E}_{i} \otimes \mathscr{F}_{j} \mathrm{BB}^{\prime}=\frac{\mathrm{A} \mathscr{E}_{i} \mathrm{~B}}{\mathrm{~A}^{\prime} \mathscr{F}_{j} \mathrm{~B}^{\prime},}
\end{aligned}
$$

and analogously for instruments.

Very relevant structures in the theory are the real space $\mathcal{H}_{\mathrm{A}}$ of Hermitian operators on $\mathrm{H}_{\mathrm{A}}$, spanned by quantum states, the cone of positive operators in $\mathcal{P}_{\mathrm{A}} \subseteq \mathcal{H}_{\mathrm{A}}$, the convex set of states $\llbracket \mathrm{A} \rrbracket$, obtained by intersecting the positive cone with the half-space $\operatorname{Tr}[X] \leq 1$, and the convex set of deterministic states $\llbracket A \rrbracket_{1}$, obtained by intersecting the positive cone with the affine hyperplane $\operatorname{Tr}[X]=1$. Similar structures can be generalised to the space spanned by quantum operations. In the general framework of OPTs, we will systematically refer to the generalisation of the above concepts.

\subsection{Formal framework}

The framework of OPTs is meant to capture the main traits of Quantum Theory (shared e.g. by Classical Theory, or Fermionic Theory, etc.) summarised above, and use them as defining properties of a family of abstract theories that might be candidates for an alternative representation of elementary physical systems and their transformations. In the remainder of this section we provide a brief review of the framework of OPTs (for reference see e.g. $[15,8,9])$. Some of the most relevant differences with respect to the quantum case stem from the fact that systems might not compose with the tensor product rule.
We warn the reader that this presentation has some elements that are slightly different from other reviews in the literature, and is tailored to ease the presentation of subsequent material. Most of the results in the present sections are new, and their proof will be given. Those theorems that are not original are not proved, and due reference is provided.

An operational theory $\Theta$ consists in i) a collection Test $(\Theta)$ of tests $\mathrm{T}_{\mathrm{X}}^{\mathrm{A} \rightarrow \mathrm{B}}$, each labelled by input and output letters from a collection $\operatorname{Sys}(\Theta)$ denoting system types, e.g. $\mathrm{A} \rightarrow \mathrm{B}$-that will be systematically omitted - and by a finite set of outcomes $\mathrm{X}$; for every pair of types $\mathrm{A}, \mathrm{B} \in \operatorname{Sys}(\Theta)$ the set of tests of type $\mathrm{A} \rightarrow \mathrm{B}$ is denoted $\langle\langle\mathrm{A} \rightarrow$ $\mathrm{B}\rangle\rangle$; ii) a very basic associative rule for sequential composition: the test $\mathrm{T}_{\mathrm{X}} \in\langle\langle\mathrm{A} \rightarrow \mathrm{B}\rangle\rangle$ can be followed by the test $\mathrm{S}_{\mathrm{Y}} \in\left\langle\left\langle\mathrm{A}^{\prime} \rightarrow \mathrm{B}^{\prime}\right\rangle\right\rangle$ if $\mathrm{A}^{\prime} \equiv \mathrm{B}$, thus obtaining the sequential composition $\mathrm{ST}_{\mathrm{X} \times \mathrm{Y}} \in$ $\left\langle\left\langle\mathrm{A} \rightarrow \mathrm{B}^{\prime}\right\rangle\right\rangle$; iii) a rule $\otimes:(\mathrm{A}, \mathrm{B}) \mapsto \mathrm{AB}$ for composing labels in parallel, and a corresponding rule for tests $\otimes:\left(\mathrm{S}_{\mathrm{X}}, \mathrm{T}_{\mathrm{Y}}\right) \mapsto(\mathrm{S} \otimes \mathrm{T})_{\mathrm{X} \times \mathrm{Y}}$, with the following properties

1. Associativity: $(\mathrm{AB}) \mathrm{C}=\mathrm{A}(\mathrm{BC})$.

2. For every $\mathrm{S}_{\mathrm{X}} \in\langle\langle\mathrm{A} \rightarrow \mathrm{B}\rangle\rangle$ and $\mathrm{T}_{\mathrm{Y}} \in\langle\langle\mathrm{C} \rightarrow$ $\mathrm{D}\rangle\rangle$, one has $\mathrm{S}_{X} \otimes \mathrm{T}_{Y} \in\langle\langle\mathrm{AC} \rightarrow \mathrm{BD}\rangle\rangle$. Associativity of $\otimes$ holds:

$$
\left(S_{X} \otimes T_{Y}\right) \otimes W_{Z}=S_{X} \otimes\left(T_{Y} \otimes W_{Z}\right)
$$

3. Unit: there is a label $\mathrm{I}$ such that $\mathrm{IA}=\mathrm{AI}=$ A for every $\mathrm{A} \in \operatorname{Sys}(\Theta)$.

4. Identity: for every $\mathrm{A} \in \operatorname{Sys}(\Theta)$, a test $\mathrm{I}_{\mathrm{A}} \in$ $\left\langle\langle A \rightarrow A\rangle\right.$ such that $\mathrm{I}_{\mathrm{B}} \mathrm{S}_{\mathrm{X}}=\mathrm{S}_{\mathrm{X}} \mathrm{I}_{\mathrm{A}}=\mathrm{S}_{\mathrm{X}}$, for every $\mathrm{S}_{\mathrm{X}} \in\langle\langle\mathrm{A} \rightarrow \mathrm{B}\rangle\rangle$.

5. For every $\mathrm{A}_{X} \in\langle\langle\mathrm{A} \rightarrow \mathrm{B}\rangle\rangle, \mathrm{B}_{Y} \in\langle\langle\mathrm{B} \rightarrow \mathrm{C}\rangle\rangle$, $\mathrm{D}_{\mathrm{Z}} \in\langle\langle\mathrm{D} \rightarrow \mathrm{E}\rangle\rangle, \mathrm{E}_{\mathrm{W}} \in\langle\langle\mathrm{E} \rightarrow \mathrm{F}\rangle\rangle$, one has

$$
\left(B_{Y} \otimes E_{W}\right)\left(A_{X} \otimes D_{Z}\right)=\left(B_{Y} A_{X}\right) \otimes\left(E_{W} D_{Z}\right) .
$$

6. Braiding: for every pair of system types A, B, there exist tests $\mathrm{S}_{\mathrm{AB}}, \mathrm{S}_{\mathrm{AB}}^{*} \in\langle\langle\mathrm{AB} \rightarrow \mathrm{BA}\rangle\rangle$ such that $\mathrm{S}_{\mathrm{BA}}^{*} \mathrm{~S}_{\mathrm{AB}}=\mathrm{S}_{\mathrm{BA}} \mathrm{S}_{\mathrm{AB}}^{*}=\mathrm{I}_{\mathrm{AB}}$, and $S_{A B}\left(A_{X} \otimes B_{Y}\right)=\left(B_{Y} \otimes A_{X}\right) S_{A B}$. Moreover,

$$
\begin{aligned}
& \mathrm{S}_{(\mathrm{AB}) \mathrm{C}}=\left(\mathrm{I}_{\mathrm{A}} \otimes \mathrm{S}_{\mathrm{BC}}\right)\left(\mathrm{S}_{\mathrm{AC}} \otimes \mathrm{I}_{\mathrm{B}}\right), \\
& \mathrm{S}_{\mathrm{A}(\mathrm{BC})}=\left(\mathrm{S}_{\mathrm{AB}} \otimes \mathrm{I}_{\mathrm{C}}\right)\left(\mathrm{I}_{\mathrm{B}} \otimes \mathrm{S}_{\mathrm{AC}}\right) .
\end{aligned}
$$

When $\mathrm{S}_{\mathrm{AB}}^{*} \equiv \mathrm{S}_{\mathrm{AB}}$, the theory is symmetric. 
All the theories developed so far are symmetric.

All tests of an operational theory are (finite) collections of events: $\langle\langle\mathrm{A} \rightarrow \mathrm{B}\rangle\rangle \ni \mathrm{R}_{\mathrm{X}}=\left\{\mathscr{R}_{i}\right\}_{i \in \mathrm{X}}$. If $\langle\langle\mathrm{A} \rightarrow \mathrm{B}\rangle\rangle \ni \mathrm{R}_{\mathrm{X}}=\left\{\mathscr{R}_{i}\right\}_{i \in \mathrm{X}}$ and $\langle\langle\mathrm{B} \rightarrow \mathrm{C}\rangle\rangle \ni$ $\mathrm{T}_{\mathrm{Y}}=\left\{\mathscr{T}_{j}\right\}_{j \in \mathrm{Y}}$, then

$$
\left\langle\langle\mathrm{A} \rightarrow \mathrm{C}\rangle \ni(\mathrm{TR})_{\mathrm{X} \times \mathrm{Y}}:=\left\{\mathscr{T}_{j} \mathscr{R}_{i}\right\}_{(i, j) \in \mathrm{X} \times \mathrm{Y}} .\right.
$$

Similarly, for $\mathrm{R}_{\mathrm{X}} \in\langle\langle\mathrm{A} \rightarrow \mathrm{B}\rangle\rangle$ and $\mathrm{T}_{\mathrm{Y}} \in\langle\langle\mathrm{C} \rightarrow$ $\mathrm{D}\rangle\rangle$,

$$
(\mathrm{R} \otimes \mathrm{T})_{\mathrm{X} \times \mathrm{Y}}:=\left\{\mathscr{R}_{i} \otimes \mathscr{T}_{j}\right\}_{(i, j) \in \mathrm{X} \times \mathrm{Y}}
$$

The set of events of tests in $\langle\langle\mathrm{A} \rightarrow \mathrm{B}\rangle\rangle$ is denoted by $\llbracket \mathrm{A} \rightarrow \mathrm{B} \rrbracket$. By the properties of sequential and parallel composition of tests, one can easily derive associativity of sequential and parallel composition of events, as well as the analogue of Eq. (1). For every test $\mathrm{T}_{\mathrm{X}} \in\langle\langle\mathrm{A} \rightarrow \mathrm{B}\rangle\rangle$ with $\mathrm{T}_{\mathbf{X}}=\left\{\mathscr{T}_{i}\right\}_{i \in \mathrm{X}}$, and every disjoint partition $\left\{\mathrm{X}_{j}\right\}_{j \in \mathrm{Y}}$ of $\mathrm{X}=\bigcup_{j \in \mathrm{Y}} \mathrm{X}_{j}$, one has a coarse graining operation that maps $\mathrm{T}_{\mathrm{X}}$ to $\mathrm{T}_{\mathrm{Y}}^{\prime} \in\langle\langle\mathrm{A} \rightarrow \mathrm{B}\rangle$, with $\mathrm{T}_{\mathrm{Y}}^{\prime}=\left\{\mathscr{T}_{j}^{\prime}\right\}_{j \in \mathrm{Y}}$. We define $\mathscr{T}_{\mathrm{X}_{j}}:=\mathscr{T}_{j}^{\prime}$. The parallel and sequential compositions distribute over coarse graining:

$$
\begin{aligned}
& \mathscr{T}_{\mathrm{X}_{j}} \otimes \mathscr{R}_{k}=(\mathscr{T} \otimes \mathscr{R})_{\mathrm{X}_{j} \times\{k\}}, \\
& \mathscr{A}_{l} \mathscr{T}_{\mathrm{X}_{j}} \mathscr{B}_{k}=(\mathscr{A} \mathscr{T} \mathscr{B})_{\{l\} \times \mathrm{X}_{j} \times\{k\}} .
\end{aligned}
$$

Notice that for every test $\mathrm{T}_{\mathrm{X}} \in\langle\langle\mathrm{A} \rightarrow \mathrm{B}\rangle\rangle$ there exists the singleton test $T_{*}^{\prime}:=\left\{\mathscr{T}_{X}\right\}$. One can easily prove that the identity test $\mathrm{I}_{\mathrm{A}}$ is a singleton: $\mathrm{I}_{\mathrm{A}}=\left\{\mathscr{I}_{\mathrm{A}}\right\}$, and $\mathscr{I}_{\mathrm{B}} \mathscr{T}=\mathscr{T} \mathscr{I}_{\mathrm{A}}$ for every event $\mathscr{T} \in \llbracket \mathrm{A} \rightarrow \mathrm{B} \rrbracket$. Similarly, for $\mathrm{S}_{\mathrm{AB}}=\left\{\mathscr{S}_{\mathrm{AB}}\right\}$ and $\mathrm{S}_{\mathrm{AB}}^{*}=\left\{\mathscr{S}_{\mathrm{AB}}^{*}\right\}$ we have $\mathscr{S}_{\mathrm{BA}}^{*} \mathscr{S}_{\mathrm{AB}}=\mathscr{S}_{\mathrm{BA}} \mathscr{S}_{\mathrm{AB}}^{*}=\mathscr{I}_{\mathrm{AB}}$. The collection of events of an operational theory $\Theta$ will be denoted by $\operatorname{Ev}(\Theta)$. The above requirements make the collections Test $(\Theta)$ and $\operatorname{Ev}(\Theta)$ the families of morphisms of two braided monoidal categories with the same objects - system types $\operatorname{Sys}(\Theta)$.

An operational theory is an OPT if the tests $\langle\langle\mathrm{I} \rightarrow \mathrm{I}\rangle\rangle$ are probability distributions: $1 \geq \mathscr{T}_{i}=$ $p_{i} \geq 0$, so that $\sum_{i \in \mathrm{X}} p_{i}=1$, and given two tests $\mathrm{S}_{\mathrm{X}}, \mathrm{T}_{\mathrm{Y}} \in\langle\langle\mathrm{I} \rightarrow \mathrm{I}\rangle\rangle$ with $\mathscr{S}_{i}=p_{i}$ and $\mathscr{T}_{i}=q_{i}$, the following identities hold

$$
\begin{aligned}
& \mathscr{S}_{i} \otimes \mathscr{T}_{j}=\mathscr{S}_{i} \mathscr{T}_{j}:=p_{i} q_{j}, \\
& \mathscr{T}_{\mathrm{X}_{j}}:=\sum_{i \in \mathrm{X}_{j}} p_{i},
\end{aligned}
$$

meaning that events in the same test are mutually exclusive and events in different tests of system I are independent. While it is immediate that $1 \in$ $\llbracket \mathrm{I} \rightarrow \mathrm{I} \rrbracket$ - since $\{1\}_{*}=\left\{\mathscr{I}_{\mathrm{I}}\right\}$ is the only singleton test-we will assume that $0 \in \llbracket I \rightarrow I \rrbracket$. This means that we can consider e.g. tests of the form $\{1,0,0\}$.

Events in $\llbracket \mathrm{A} \rightarrow \mathrm{B} \rrbracket$ are called transformations. As a consequence of the above definitions, every set $\llbracket \mathrm{A} \rrbracket:=\llbracket \mathrm{I} \rightarrow \mathrm{A} \rrbracket$ can be viewed as a set of functionals on $\llbracket \bar{A} \rrbracket$. As such, it can be viewed as a spanning subset of the real vector space $\llbracket A \rrbracket_{\mathbb{R}}$ of linear funcitonals on $\llbracket \overline{\mathrm{A}} \rrbracket$. On the other hand $\llbracket \overline{\mathrm{A}} \rrbracket:=\llbracket \mathrm{A} \rightarrow \mathrm{I} \rrbracket$ is a separating set of positive linear functionals on $\llbracket \mathrm{A} \rrbracket$, which then spans the dual space $\llbracket \mathrm{A} \rrbracket_{\mathbb{R}}^{*}=: \llbracket \overline{\mathrm{A}} \rrbracket_{\mathbb{R}}$. The dimension $D_{\mathrm{A}}$ of $\llbracket A \rrbracket_{\mathbb{R}}$ (which is the same as that of $\llbracket \bar{A} \rrbracket_{\mathbb{R}}$ ) is called size of system A. One can easily prove that in any OPT $\Theta, \mathrm{I}$ is the unique system with unit size $D_{\mathrm{I}}=1$. Using the properties of parallel composition, one can also prove that $D_{\mathrm{AB}} \geq D_{\mathrm{A}} D_{\mathrm{B}}$. Events in $\llbracket \mathrm{A} \rrbracket$ are called states, and denoted by lower-case greek letters, e.g. $\rho$, while events in $\llbracket \overline{\mathrm{A}} \rrbracket$ are called effects, and denoted by lower-case latin letters, e.g. $a$. When it is appropriate, we will use the symbol $\mid \rho)$ to denote a state, and $(a \mid$ to denote an effect. We will also use the circuit notation, where we denote states, transformations and effects by the symbols

$$
\rho \mathrm{A}, \mathrm{A} \mathscr{A} \mathrm{B}, \mathrm{A} a,
$$

respectively. Sequential composition of $\mathscr{A} \in$ $\llbracket \mathrm{A} \rightarrow \mathrm{B} \rrbracket$ and $\mathscr{B} \in \llbracket \mathrm{B} \rightarrow \mathrm{C} \rrbracket$ is denoted by the diagram

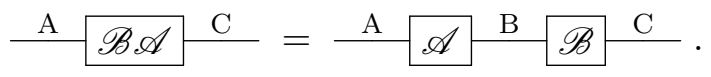

For composite systems we use diagrams with multiple wires, e.g.

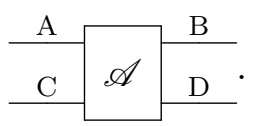

The identity will be omitted: A $\mathscr{I}$ A $=$ A . The swap $\mathscr{S}_{\mathrm{AB}}$ and its inverse will be denoted as follows
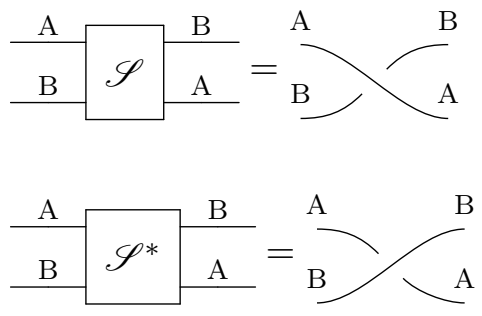
In the present paper we will always assume that the theory under consideration is symmetric, however all the results will be straightforwardly generalisable. We will consequently draw the swap $\mathscr{S}_{\mathrm{AB}}$ as

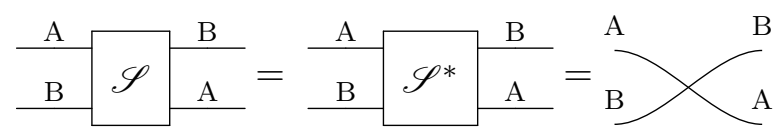

An OPT $\Theta$ is specified by the collections of systems and tests, along with the parallel composition rule $\otimes$

$$
\Theta \equiv(\operatorname{Test}(\Theta), \operatorname{Sys}(\Theta), \otimes) .
$$

Definition 1 (Equal transformations). Let $\mathscr{A}, \mathscr{B} \in \llbracket \mathrm{A} \rightarrow \mathrm{B} \rrbracket$. Then we define $\mathscr{A}=\mathscr{B}$ if for every system $\mathrm{C}$ and every $\rho \in \llbracket \mathrm{AC} \rrbracket$ and $a \in \llbracket \overline{\mathrm{B}} \overline{\mathrm{C}} \rrbracket$, one has

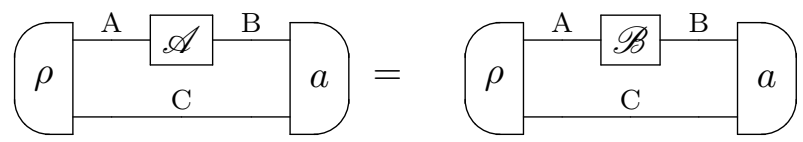

Notice that, since states separate effects and viceversa effects separate states, the above definition is equivalent to the two following equality criteria.

Lemma 1. Let $\mathscr{A}, \mathscr{B} \in \llbracket \mathrm{A} \rightarrow \mathrm{B} \rrbracket$. Then the following conditions are equivalent.

1. $\mathscr{A}=\mathscr{B}$.

2. For every $\mathrm{C}$ and every $\rho \in \llbracket \mathrm{AC} \rrbracket$, one has

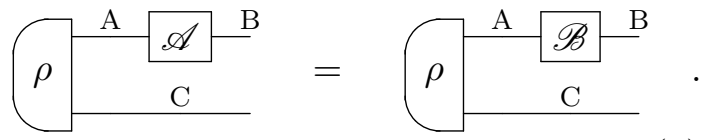

3. For every $\mathrm{C}$ and every $a \in \llbracket \overline{\mathrm{B}} \overline{\mathrm{C}} \rrbracket$, one has

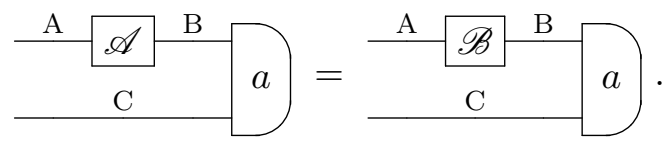

One can show [8] that events $\mathscr{T} \in \llbracket \mathrm{A} \rightarrow \mathrm{B} \rrbracket$ can be identified with a family of linear maps, one for every $\mathrm{C}$, that characterize the action of $\mathscr{T} \otimes \mathscr{I}_{\mathrm{C}}$ on $\llbracket \mathrm{AC} \rrbracket_{\mathbb{R}}$ as a linear map to $\llbracket \mathrm{BC} \rrbracket_{\mathbb{R}}$. As anticipated in the introductory paragraph, we remind the reader that some of the difficulties that we will be faced with in the remainder originate from the fact that in a general theory it is not true that $\llbracket \mathrm{AB} \rrbracket_{\mathbb{R}}=\llbracket \mathrm{A} \rrbracket_{\mathbb{R}} \otimes \llbracket \mathrm{B} \rrbracket_{\mathbb{R}}$, but only $\llbracket \mathrm{A} \rrbracket_{\mathbb{R}} \otimes \llbracket \mathrm{B} \rrbracket_{\mathbb{R}} \subseteq \llbracket \mathrm{AB} \rrbracket_{\mathbb{R}}$. As a consequence, the linear map representing $\mathscr{T}$ on $\llbracket \mathrm{A} \rrbracket_{\mathbb{R}}$ is not sufficient to determine the linear map representing $\mathscr{T} \otimes \mathscr{I}_{\mathrm{C}}$ on $\llbracket A C \rrbracket_{\mathbb{R}}$.

One can easily prove that $\llbracket A \rightarrow B \rrbracket$ spans a real vector space $\llbracket \mathrm{A} \rightarrow \mathrm{B} \rrbracket_{\mathbb{R}}$. Being $\llbracket \mathrm{A} \rightarrow \mathrm{B} \rrbracket$ spanning for $\llbracket \mathrm{A} \rightarrow \mathrm{B} \rrbracket_{\mathbb{R}}$, the criteria of definition 1 and lemma 1 hold for $\mathscr{A}, \mathscr{B} \in \llbracket \mathrm{A} \rightarrow \mathrm{B} \rrbracket_{\mathbb{R}}$. Elements of $\llbracket \mathrm{A} \rightarrow \mathrm{B} \rrbracket_{\mathbb{R}}$ are called generalized events. Every space $\llbracket \mathrm{A} \rightarrow \mathrm{B} \rrbracket_{\mathbb{R}}$ has a zero event $0_{\mathrm{A} \rightarrow \mathrm{B}}:=0_{\mathrm{I} \rightarrow \mathrm{I}} \otimes$ $\mathscr{T} \in \llbracket \mathrm{A} \rightarrow \mathrm{B} \rrbracket$, where $\mathscr{T}$ is an arbitrary event in $\llbracket \mathrm{A} \rightarrow \mathrm{B} \rrbracket$. As a consequence of the coarse graining rule for tests on $\llbracket I \rightarrow I \rrbracket$, one can easily show that coarse graining of two or more transformations is represented by their sum. Precisely, given a test $\left\{\mathscr{T}_{i}\right\}_{i \in \mathrm{X}} \subseteq \llbracket \mathrm{A} \rightarrow \mathrm{B} \rrbracket$, for $\mathrm{X}_{0}=\{0,1\} \subseteq \mathrm{X}$ one has $\mathscr{T}_{0}^{\prime}=\mathscr{T}_{0}+\mathscr{T}_{1}$, namely for every system $\mathrm{C}$ it is $\mathscr{T}_{0}^{\prime} \otimes \mathscr{I}_{\mathrm{C}}=\mathscr{T}_{0} \otimes \mathscr{I}_{\mathrm{C}}+\mathscr{T}_{1} \otimes \mathscr{I}_{\mathrm{C}}$. Finally, every set $\llbracket \mathrm{A} \rightarrow \mathrm{B} \rrbracket$ has a subset consisting in singleton events, that we denote by $\llbracket \mathrm{A} \rightarrow \mathrm{B} \rrbracket_{1}$, and call deterministic. For $\mathrm{A}, \mathrm{B} \neq \mathrm{I}$, a deterministic event is called channel. A channel $\mathscr{U} \in \llbracket \mathrm{A} \rightarrow \mathrm{B} \rrbracket_{1}$ is reversible if there exists a channel $\mathscr{V} \in \llbracket \mathrm{B} \rightarrow \mathrm{A} \rrbracket_{1}$ such that $\mathscr{V} \mathscr{U}=\mathscr{I}_{\mathrm{A}}, \mathscr{U} \mathscr{V}=\mathscr{I}_{\mathrm{B}}$.

Let us now define the cones

$$
\llbracket \mathrm{A} \rightarrow \mathrm{B} \rrbracket_{+}:=\{\lambda \mathscr{T} \mid \lambda \geq 0, \mathscr{T} \in \llbracket \mathrm{A} \rightarrow \mathrm{B} \rrbracket\} .
$$

We will often write $\mathscr{A} \triangleright 0$ as a shorthand for $\mathscr{A} \in \llbracket \mathrm{A} \rightarrow \mathrm{B} \rrbracket_{+}$. The cone $\llbracket \mathrm{A} \rightarrow \mathrm{B} \rrbracket_{+}$introduces a partial ordering in $\llbracket \mathrm{A} \rightarrow \mathrm{B} \rrbracket_{\mathbb{R}}$, defined by

$$
\mathscr{A} \triangleright \mathscr{B} \quad \Leftrightarrow \quad(\mathscr{A}-\mathscr{B}) \triangleright 0 .
$$

OPTs are assumed to have all sets $\llbracket \mathrm{A} \rightarrow \mathrm{B} \rrbracket$ (and thus also cones $\left.\llbracket \mathrm{A} \rightarrow \mathrm{B} \rrbracket_{+}\right)$closed in the operational norm.

Two systems may be operationally equivalent if they can be mapped one onto the other via a reversible transformation. Clearly, in this case every processing of the first system is perfectly simulated by a processing of the other, and viceversa.We define operationally equivalent systems as follows.

Definition 2. Let A, B be two systems. We say that $\mathrm{A}$ and $\mathrm{B}$ are operationally equivalent, in formula $\mathrm{A} \cong \mathrm{B}$, if there exists a reversible transformation $\mathscr{U} \in \llbracket \mathrm{A} \rightarrow \mathrm{B} \rrbracket_{1}$.

Definition 3. If $\mathrm{A}_{1}$ and $\mathrm{A}_{2}$ are operationally equivalent through $\mathscr{U}$ and $\mathrm{B}_{1}$ and $\mathrm{B}_{2}$ through $\mathscr{V}$, 
then, for every system $\mathrm{C}, \mathscr{A}_{1} \in \llbracket \mathrm{A}_{1} \mathrm{C} \rightarrow \mathrm{B}_{1} \mathrm{C} \rrbracket_{\mathbb{R}}$ and $\mathscr{A}_{2} \in \llbracket \mathrm{A}_{2} \mathrm{C} \rightarrow \mathrm{B}_{2} \mathrm{C} \rrbracket_{\mathbb{R}}$ are operationally equivalent if $\mathscr{A}_{2}=\left(\mathscr{V} \otimes \mathscr{I}_{\mathrm{C}}\right) \mathscr{A}_{1}\left(\mathscr{U}^{-1} \otimes \mathscr{I}_{\mathrm{C}}\right)$. In particular, $\rho_{1} \in \llbracket \mathrm{A}_{1} \mathrm{C} \rrbracket_{\mathbb{R}}$ and $\rho_{2} \in \llbracket \mathrm{A}_{2} \mathrm{C} \rrbracket_{\mathbb{R}}$ are operationally equivalent if $\rho_{2}=\left(\mathscr{U} \otimes \mathscr{I}_{\mathrm{C}}\right) \rho_{1}$, and $a_{1} \in \llbracket \overline{\mathrm{A}}_{1} \overline{\mathrm{C}} \rrbracket_{\mathbb{R}}$ and $a_{2} \in \llbracket \overline{\mathrm{A}}_{2} \overline{\mathrm{C}} \rrbracket_{\mathbb{R}}$ are operationally equivalent if $a_{2}=a_{1}\left(\mathscr{U}^{-1} \otimes \mathscr{I}_{\mathrm{C}}\right)$.

Lemma 2. Let $\mathrm{AB}$ be operationally equivalent to A. Then $\mathrm{B}$ must be operationally equivalent to the trivial system $\mathrm{I}$.

Proof. First of all, since $D_{\mathrm{AB}} \geq D_{\mathrm{A}} D_{\mathrm{B}} \geq D_{\mathrm{A}}$, if $\mathrm{AB} \cong \mathrm{A}$ the chain of inequalities is saturated, and thus $D_{\mathrm{B}}=1$. This implies that every state of system $\mathrm{AB}$ is of the form $\tau \otimes 1_{\mathrm{B}}$, where $\tau \in \llbracket \mathrm{A} \rrbracket$ and $1_{\mathrm{B}}$ is the unique deterministic state of system B. The same result holds for states of $\mathrm{ABC}$ for arbitrary C. Now, let $\mathscr{U} \in \llbracket \mathrm{AB} \rightarrow \mathrm{A} \rrbracket_{1}$ be the reversible channel implementing operational equivalence. Let $\eta \in \llbracket \mathrm{AC} \rrbracket$, and $\left(\mathscr{U} \otimes \mathscr{I}_{\mathrm{C}}\right)\left(1_{\mathrm{B}} \otimes \eta\right)=\tilde{\eta} \in \llbracket \mathrm{AC} \rrbracket$. One can choose $\mathscr{U}$ as to identify $\tilde{\eta}=\eta$. Indeed, there is a reversible transformation $\mathscr{V} \in \llbracket \mathrm{A} \rightarrow \mathrm{A} \rrbracket$ such that $\left(\mathscr{V} \otimes \mathscr{I}_{\mathrm{C}}\right) \tilde{\eta}=\eta$. In particular, one can choose $\mathscr{V}:=1_{\mathrm{B}} \mathscr{U}^{-1}$, where now 1 represents the unique deterministic effect of B. Rightreversibility of $\mathscr{V}$ is trivial. On the other hand, let $\sigma \in \llbracket \mathrm{AC} \rrbracket$, and let $1_{\mathrm{B}} \otimes \tau:=\left(\mathscr{U}^{-1} \otimes \mathscr{I}_{\mathrm{C}}\right) \sigma \in$ $\llbracket A B C \rrbracket$. Now, after applying the deterministic effect $1_{\mathrm{B}}$ one has $\left(\mathscr{V} \otimes \mathscr{I}_{\mathrm{C}}\right) \sigma=\tau$. One can reverse $\mathscr{V}$ by composing $\tau$ with $1_{\mathrm{B}}$, obtaining $1_{\mathrm{B}} \otimes \tau$, and then applying $\mathscr{U} \otimes \mathscr{I}_{\mathrm{C}}$, finally obtaining $\left(\mathscr{U} \otimes \mathscr{I}_{\mathrm{C}}\right)\left(1_{\mathrm{B}} \otimes \tau\right)=\sigma$. With a suitable choice of $\mathscr{U}$, one then has $\left(\mathscr{U} \otimes \mathscr{I}_{\mathrm{C}}\right)\left(1_{\mathrm{B}} \otimes \eta\right)=\eta$. Let now $1_{\mathrm{B}} \otimes \eta=\tau_{\mathrm{BC}} \otimes \nu_{\mathrm{A}}$, with $\nu \in \llbracket \mathrm{A} \rrbracket_{1}$. Discarding system $\mathrm{A}$ on both sides via some deterministic effect $e_{\mathrm{A}} \in \llbracket \overline{\mathrm{A}} \rrbracket_{1}$, one obtains $\tau_{\mathrm{BC}}=1_{\mathrm{B}} \otimes \theta$, with $\theta=\left(e_{\mathrm{A}} \otimes \mathscr{I}_{\mathrm{C}}\right) \eta$. Finally, this implies that every state of system $\mathrm{BC}$ is of the form $\tau_{\mathrm{BC}}=1_{\mathrm{B}} \otimes \theta$, with $\theta \in \llbracket \mathrm{C} \rrbracket$. Once we fix $\eta \in \llbracket \mathrm{A} \rrbracket_{1}$, we can then define the reversible channel $\mathscr{W} \in \llbracket \mathrm{BC} \rightarrow \mathrm{C} \rrbracket$ given by $\mathscr{W}:=e_{\mathrm{A}}\left(\mathscr{U} \otimes \mathscr{I}_{\mathrm{C}}\right)\left(\eta \otimes \mathscr{I}_{\mathrm{BC}}\right)$. Clearly, $\mathscr{W}\left(1_{\mathrm{B}} \otimes \theta\right)=\theta$, and the inverse map $\mathscr{W}^{-1} \in \llbracket \mathrm{C} \rightarrow$ $\mathrm{BC} \rrbracket$ is obtained as $\mathscr{W}^{-1}:=e_{\mathrm{A}}\left(\mathscr{U}^{-1} \eta \otimes \mathscr{I}_{\mathrm{C}}\right)$. We then have $\mathrm{BC} \simeq \mathrm{C}$ for every $\mathrm{C}$, i.e. $\mathrm{B} \simeq \mathrm{I}$.

\subsection{Causality and the no-restriction hypothesis}

In the remainder of the paper we will focus on causal theories. The causality property, that we define right away, characterizes those theories where signals can propagate only in the direction defined by input and output of processes, within a cone of causal influence determined by interactions between systems. These are the only theories where one can consistently use information acquired in a set of tests to condition the choice of subsequent tests, where the partial ordering we are referring to is that determined by the input/output direction.

Definition 4 (Causal theories). A theory $(\mathscr{T}, \mathrm{A})$ is causal if for every test $\left\{\mathscr{T}_{i}\right\}_{i \in \mathrm{X}}$ and every collection of tests $\left\{\mathscr{S}_{j}^{i}\right\}_{j \in \mathrm{Y}}$ labelled by $i \in \mathrm{Y}$, the generalized test $\left\{\mathscr{C}_{i, j}\right\}_{(i, j) \in \mathrm{X} \times \mathrm{Y}}$ with

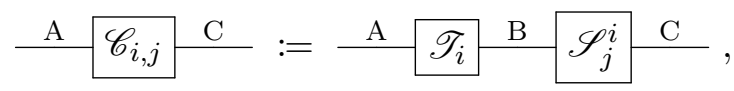

is a test of the theory.

Notice that this notion of causality is strictly stronger than the one usually adopted in the literature about OPTs, that can be summarised as uniqueness of the deterministic effect (see e.g. Refs. $[8,9,15])$. Indeed, a first result of crucial importance derives uniqueness of the deterministic effect for every system type in causal theories.

Theorem 1 ([8, 15]). In a causal theory, for every system type $\mathrm{A}$ the set of deterministic effects $\llbracket \overline{\mathrm{A}} \rrbracket_{1}$ is the singleton $\left\{e_{\mathrm{A}}\right\}$.

A proof of the above theorem, that proceeds by contradiction, can be found in the mentioned references. We remark that, being the notion of causality in these references different form the one defined here, the theorem has a slightly different statement. In the same references, one can find the equivalence of uniqueness of the deterministic effect with non-signalling from output to input.

Theorem $2([8,15])$. In an OPT, every system type $\mathrm{A}$ has a unique deterministic effect if and only if the marginal probabilities of preparation tests cannot depend on the choice of subsequent observation tests.

As a consequence of our notion of causality, in a non-deterministic theory (i.e. a theory where $\llbracket \mathrm{I} \rrbracket \neq\{0,1\})$, every convex combination of tests is a test (see e.g. [15]). This implies that the sets $\llbracket \mathrm{A} \rrbracket_{*}, \llbracket \overline{\mathrm{A}} \rrbracket_{*}, \llbracket \mathrm{A} \rightarrow \mathrm{B} \rrbracket_{*}$ are convex, where $*$ can be replaced by nothing, 1 or + . Moreover, the sets with $*=+$ are convex cones. 
Another important consequence of causality is that a transformation $\mathscr{C} \in \llbracket \mathrm{A} \rightarrow \mathrm{B} \rrbracket$ is deterministic if and only if it maps the deterministic effect $e_{\mathrm{B}}$ to the deterministic effect $e_{\mathrm{A}}$.

Theorem $3([8,15])$. In a causal theory, a transformation $\mathscr{C} \in \llbracket \mathrm{A} \rightarrow \mathrm{B} \rrbracket$ is a channel iff

$$
\mathrm{A} \mathscr{C} \mathrm{B}=\mathrm{A} e \mathrm{e} .
$$

In causal theories, one can always assume that every state is proportional to a deterministic one. Indeed, including in the theory every state $\rho \in$ $\llbracket \mathrm{A} \rrbracket+$ such that $\left(e_{\mathrm{A}} \mid \rho\right)=1$ does not introduce any inconsistency with the set of transformations, as we now prove. Let $\Theta$ be a causal OPT, and define the theory $\Theta^{\prime}$ through the bijection $\kappa$ : Sys $(\Theta) \rightarrow$ Sys $\left(\Theta^{\prime}\right):: \mathrm{A} \mapsto \mathrm{A}^{\prime}$, with

$$
\begin{aligned}
& \llbracket \mathrm{A}^{\prime} \rightarrow \mathrm{B}^{\prime} \rrbracket_{\mathbb{R}} \equiv \llbracket \mathrm{A} \rightarrow \mathrm{B} \rrbracket_{\mathbb{R}}, \\
& \llbracket \mathrm{A}^{\prime} \rightarrow \mathrm{B}^{\prime} \rrbracket_{1}:=\left\{\mathscr{T} \triangleright 0 \mid\left(e_{\mathrm{B}} \mid \mathscr{T}=\left(e_{\mathrm{A}} \mid\right\},\right.\right. \\
& \llbracket \mathrm{A}^{\prime} \rightarrow \mathrm{B}^{\prime} \rrbracket:=\left\{\mathscr{T} \triangleright 0 \mid \exists \mathscr{C} \in \llbracket \mathrm{A}^{\prime} \rightarrow \mathrm{B}^{\prime} \rrbracket_{1}, \mathscr{C} \triangleright \mathscr{T}\right\}, \\
& \left\langle\left\langle\mathrm{A}^{\prime} \rightarrow \mathrm{B}^{\prime}\right\rangle\right\rangle:= \\
& \quad\left\{\mathrm{T} \subseteq \llbracket \mathrm{A}^{\prime} \rightarrow \mathrm{B}^{\prime} \rrbracket \mid \sum_{\mathscr{T} \in \mathrm{T}} \mathscr{T} \in \llbracket \mathrm{A}^{\prime} \rightarrow \mathrm{B}^{\prime} \rrbracket_{1}\right\} .
\end{aligned}
$$

where $\triangleright$ denotes the ordering induced by the cones of the theory $\Theta$, and the cardinality of sets T in the last definition is implicitly assumed to be finite.

Theorem 4. Let $\Theta$ be a causal OPT, and consider the theory $\Theta^{\prime}$ defined above. Then $\Theta^{\prime}$ is an OPT with a unique deterministic effect $e_{\mathrm{A}^{\prime}}$ for every $\mathrm{A}^{\prime}$.

Proof. All the compositional structures of $\Theta$ are inherited by $\Theta^{\prime}$. The defining conditions in the special case of $\llbracket \mathrm{A}^{\prime} \rrbracket=\llbracket \mathrm{I}^{\prime} \rightarrow \mathrm{A}^{\prime} \rrbracket$ give

$$
\llbracket \mathrm{A}^{\prime} \rrbracket=\left\{\rho \in \llbracket \mathrm{A} \rrbracket+\mid\left(e_{\mathrm{A}} \mid \rho\right) \leq 1\right\},
$$

while for $\llbracket \bar{A}^{\prime} \rrbracket=\llbracket \mathrm{A}^{\prime} \rightarrow \mathrm{I}^{\prime} \rrbracket$ they give

$$
\llbracket \overline{\mathrm{A}}^{\prime} \rrbracket_{1}=\left\{e_{\mathrm{A}}\right\} .
$$

What remains to be proved is that the set of transformations is closed under sequential and parallel composition. First of all, we observe that by theorem $3 \llbracket \mathrm{A} \rightarrow \mathrm{B} \rrbracket_{1} \subseteq \llbracket \mathrm{A}^{\prime} \rightarrow \mathrm{B}^{\prime} \rrbracket_{1}$, thus $\llbracket \mathrm{A} \rightarrow \mathrm{B} \rrbracket \subseteq \llbracket \mathrm{A}^{\prime} \rightarrow \mathrm{B}^{\prime} \rrbracket$. This makes $\llbracket \mathrm{A} \rightarrow \mathrm{B} \rrbracket_{+} \subseteq$ $\llbracket \mathrm{A}^{\prime} \rightarrow \mathrm{B}^{\prime} \rrbracket_{+}$. On the other hand, since by definition it is also $\llbracket \mathrm{A}^{\prime} \rightarrow \mathrm{B}^{\prime} \rrbracket \subseteq \llbracket \mathrm{A} \rightarrow \mathrm{B} \rrbracket_{+}$, we have $\llbracket \mathrm{A}^{\prime} \rightarrow \mathrm{B}^{\prime} \rrbracket_{+} \subseteq \llbracket \mathrm{A} \rightarrow \mathrm{B} \rrbracket_{+}$, which makes $\llbracket \mathrm{A}^{\prime} \rightarrow \mathrm{B}^{\prime} \rrbracket_{+} \equiv \llbracket \mathrm{A} \rightarrow \mathrm{B} \rrbracket_{+}$. Thus the ordering $\triangleright$ is the same for the two theories. As a consequence, the preservation of cones under the two compositions is inherited by $\Theta^{\prime}$ from the same property in $\Theta$. Now, if $\mathscr{A} \in \llbracket \mathrm{A}^{\prime} \rightarrow \mathrm{B}^{\prime} \rrbracket_{1}$ and $\mathscr{B} \in \llbracket \mathrm{B}^{\prime} \rightarrow \mathrm{C}^{\prime} \rrbracket_{1}$, then

$$
\left(e_{\mathrm{C}} \mid \mathscr{B} \mathscr{A}=\left(e_{\mathrm{B}} \mid \mathscr{A}=\left(e_{\mathrm{A}} \mid,\right.\right.\right.
$$

thus $\mathscr{B} \mathscr{A} \in \llbracket \mathrm{A}^{\prime} \rightarrow \mathrm{C}^{\prime} \rrbracket_{1}$. Moreover, thanks to causality of the theory $\Theta$ one has $e_{\mathrm{AB}}=e_{\mathrm{A}} \otimes e_{\mathrm{B}}$, thus for $\mathscr{A} \in \llbracket \mathrm{A}^{\prime} \rightarrow \mathrm{B}^{\prime} \rrbracket_{1}$ and $\mathscr{B} \in \llbracket \mathrm{C}^{\prime} \rightarrow \mathrm{D}^{\prime} \rrbracket_{1}$,

$$
\begin{aligned}
\left(e_{\mathrm{BD}} \mid \mathscr{A} \otimes \mathscr{B}\right. & =\left(e_{\mathrm{B}} \mid \mathscr{A} \otimes\left(e_{\mathrm{D}} \mid \mathscr{B}\right.\right. \\
& =\left(e_{\mathrm{A}} \mid \otimes\left(e_{\mathrm{C}} \mid=\left(e_{\mathrm{AC}} \mid,\right.\right.\right.
\end{aligned}
$$

and consequently $\mathscr{A} \otimes \mathscr{B} \in \llbracket \mathrm{A}^{\prime} \mathrm{C}^{\prime} \rightarrow \mathrm{B}^{\prime} \mathrm{D}^{\prime} \rrbracket_{1}$. Now, given events $\mathscr{A}, \mathscr{B}$ in the theory $\Theta^{\prime}$, by definition we have $\mathscr{C}, \mathscr{D}$ deterministic in $\Theta^{\prime}$ such that $\mathscr{C} \triangleright \mathscr{A}$ and $\mathscr{D} \triangleright \mathscr{B}$, thus

$$
\begin{aligned}
\mathscr{F}:= & (\mathscr{D}-\mathscr{B})(\mathscr{C}-\mathscr{A})+(\mathscr{D}-\mathscr{B}) \mathscr{A} \\
& +\mathscr{B}(\mathscr{C}-\mathscr{A}) \triangleright 0, \\
\mathscr{G}:= & (\mathscr{C}-\mathscr{A}) \otimes(\mathscr{D}-\mathscr{B}) \\
& +(\mathscr{C}-\mathscr{A}) \otimes \mathscr{B}+\mathscr{A} \otimes(\mathscr{D}-\mathscr{B}) \triangleright 0 .
\end{aligned}
$$

Finally, $\mathscr{F}+\mathscr{B} \mathscr{A} \triangleright \mathscr{B} \mathscr{A}$ and $\mathscr{G}+\mathscr{A} \otimes \mathscr{B} \triangleright \mathscr{A} \otimes$ $\mathscr{B}$ are channels, and thus $\mathscr{B} \mathscr{A}$ and $\mathscr{A} \otimes \mathscr{B}$ are events.

One can now show that the new theory $\Theta^{\prime}$ is causal.

Corollary 1. Let $\Theta$ be a causal OPT, and $\Theta^{\prime}$ as in theorem 4. Then $\Theta^{\prime}$ is a causal OPT.

Proof. Let $\left\{\mathscr{T}_{i}\right\}_{i \in \mathrm{X}}$ be a test in $\llbracket \mathrm{A}^{\prime} \rightarrow \mathrm{B}^{\prime} \rrbracket$, and $\left\{\mathscr{S}_{j}^{i}\right\}_{j \in \mathrm{Y}}$ be tests in $\llbracket \mathrm{B}^{\prime} \rightarrow \mathrm{C}^{\prime} \rrbracket$ for every $i \in \mathrm{X}$. Then

$\forall i \in \mathrm{X} \sum_{j \in \mathrm{Y}}\left(e_{\mathrm{C}^{\prime}} \mid \mathscr{S}_{j}^{i}=\left(e_{\mathrm{B}^{\prime}} \mid, \quad \sum_{i \in \mathrm{X}}\left(e_{\mathrm{B}^{\prime}} \mid \mathscr{T}_{i}=\left(e_{\mathrm{A}^{\prime}} \mid\right.\right.\right.\right.$.

This implies that

$$
\begin{aligned}
& \sum_{i \in \mathrm{X}} \sum_{j \in \mathrm{Y}}\left(e_{\mathrm{C}^{\prime}} \mid \mathscr{W}_{i, j}=\left(e_{\mathrm{A}^{\prime}} \mid,\right.\right. \\
& \mathscr{W}_{i, j}:=\mathscr{S}_{j}^{i} \mathscr{T}_{i} \in \llbracket \mathrm{A}^{\prime} \rightarrow \mathrm{C}^{\prime} \rrbracket \forall i \in \mathrm{X}, j \in \mathrm{Y} .
\end{aligned}
$$

As a consequence, every conditional test $\mathscr{W}_{i, j}:=$ $\mathscr{S}_{j}^{i} \mathscr{T}_{i} \in \llbracket \mathrm{A}^{\prime} \rightarrow \mathrm{C}^{\prime} \rrbracket$ is admitted in the theory $\Theta^{\prime}$. 
Remark 1. In the remainder, we will focus on theories satisfying causality and the further requirements

$\llbracket \mathrm{A} \rightarrow \mathrm{B} \rrbracket_{1}=\left\{\mathscr{T} \triangleright 0 \mid\left(e_{\mathrm{B}} \mid \mathscr{T}=\left(e_{\mathrm{A}} \mid\right\}\right.\right.$, $\llbracket \mathrm{A} \rightarrow \mathrm{B} \rrbracket=\left\{\mathscr{T} \triangleright 0 \mid \exists \mathscr{C} \in \llbracket \mathrm{A} \rightarrow \mathrm{B} \rrbracket_{1}, \mathscr{C} \triangleright \mathscr{T}\right\}$, $\langle\langle\mathrm{A} \rightarrow \mathrm{B}\rangle\rangle=\left\{\mathrm{T} \subseteq \llbracket \mathrm{A} \rightarrow \mathrm{B} \rrbracket \mid \sum_{\mathscr{T} \in \mathrm{T}} \mathscr{T} \in \llbracket \mathrm{A} \rightarrow \mathrm{B} \rrbracket_{1}\right\}$.

In particular, this implies that $\llbracket \mathrm{A} \rrbracket=\left\{\rho \in \llbracket \mathrm{A} \rrbracket_{+} \mid\right.$ $\left.\left(e_{\mathrm{A}} \mid \rho\right) \leq 1\right\}$. Thanks to corollary 1 , this is not a significant restriction.

We now narrow down focus on theories that satisfy the no-restriction hypothesis. Let us consider the set of preparation-tests for every system of the theory $\Theta$. Then we will complete the set of tests allowing for all those tests that transform preparation tests to preparation tests, even when applied locally. This requirement is the generalisation of the assumption made in quantum theory that a map is a transformation if and only if it is completely positive and trace non increasing, and a collection of transformations is a test if and only if the sum of its elements is a channel, i.e. completely positive and trace-preserving.

Assumption 1. Let $\mathscr{A} \in \llbracket \mathrm{A} \rightarrow \mathrm{B} \rrbracket_{\mathbb{R}}$. The norestriction hypothesis consists in the requirement that $\mathrm{T} \in\langle\langle\mathrm{A} \rightarrow \mathrm{B}\rangle$ if and only if for every $\mathrm{C}$ and every $\mathrm{P} \in\left\langle\langle\mathrm{I} \rightarrow \mathrm{AC}\rangle\right.$, one has $\left(\mathrm{T} \otimes \mathrm{I}_{\mathrm{C}}\right) \mathrm{P} \in\langle\langle\mathrm{I} \rightarrow$ $\mathrm{BC}\rangle$.

We will write $\mathscr{A} \succeq 0$ if for every $\mathrm{C}$ and every $P \in \llbracket \mathrm{AC} \rrbracket_{+}$, one has $\left(\mathscr{A} \otimes \mathscr{I}_{\mathrm{C}}\right) P \in \llbracket \mathrm{BC} \rrbracket_{+}$. The set of transformations $\mathscr{A} \succeq 0$ is a cone, that we denote by

$$
\mathrm{K}(\mathrm{A} \rightarrow \mathrm{B}):=\left\{\mathscr{A} \in \llbracket \mathrm{A} \rightarrow \mathrm{B} \rrbracket_{\mathbb{R}} \mid \mathscr{A} \succeq 0\right\} .
$$

The above defined cone introduces a (partial) ordering in $\llbracket \mathrm{A} \rightarrow \mathrm{B} \rrbracket_{\mathbb{R}}$, defined by

$$
\mathscr{A} \succeq \mathscr{B} \quad \Leftrightarrow \mathscr{A}-\mathscr{B} \succeq 0 .
$$

Notice that, under the no-restriction hypothesis, the following identity holds

$$
\mathrm{K}(\mathrm{A} \rightarrow \mathrm{B})=\llbracket \mathrm{A} \rightarrow \mathrm{B} \rrbracket_{+},
$$

and thus for every $\mathscr{A}, \mathscr{B} \in \llbracket \mathrm{A} \rightarrow \mathrm{B} \rrbracket_{\mathbb{R}}$,

$$
\mathscr{A} \succeq \mathscr{B} \Leftrightarrow \mathscr{A} \triangleright \mathscr{B} \text {. }
$$

We remark that, under assumption 1 , the hypothesis of theorem 3 can be relaxed to $\mathscr{C} \in$ $\llbracket \mathrm{A} \rightarrow \mathrm{B} \rrbracket_{+}$, since the latter implies that $\mathscr{C}=\lambda \mathscr{C}_{0}$ for some $\mathscr{C}_{0} \in \llbracket \mathrm{A} \rightarrow \mathrm{B} \rrbracket$ and $\lambda \geq 0$. Then, $\mathscr{C} \succeq 0$. Moreover, since $\mathscr{C}$ sends $e_{\mathrm{B}}$ to $e_{\mathrm{A}}$, one has $\left(\mathscr{C} \otimes \mathscr{I}_{\mathrm{C}}\right) \llbracket \mathrm{AC} \rrbracket_{1} \subseteq \llbracket \mathrm{BC} \rrbracket_{1}$, and thus $\left(\mathscr{C} \otimes \mathscr{I}_{\mathrm{C}}\right) \llbracket \mathrm{AC} \rrbracket \subseteq \llbracket \mathrm{BC} \rrbracket$. By the no-restriction hypothesis, this is tantamount to $\mathscr{C} \in \llbracket \mathrm{A} \rightarrow \mathrm{B} \rrbracket$.

We can now prove a theorem that is a very important consequence of the no-restriction hypothesis, along with causality.

Theorem 5. In a theory satisfying the norestriction hypothesis, let $\mathscr{T} \in \llbracket \mathrm{A} \rightarrow \mathrm{B} \rrbracket_{\mathbb{R}}$. Then $\mathscr{T}, \mathscr{C}-\mathscr{T} \succeq 0$ for some channel $\mathscr{C} \in \llbracket \mathrm{A} \rightarrow \mathrm{B} \rrbracket_{1}$ iff $\mathscr{T} \in \llbracket \mathrm{A} \rightarrow \mathrm{B} \rrbracket$.

Proof. The hypothesis $\mathscr{T}, \mathscr{C}-\mathscr{T} \succeq 0$ is equivalent to $\mathscr{T}, \mathscr{C}-\mathscr{T} \in \llbracket \mathrm{A} \rightarrow \mathrm{B} \rrbracket_{+}$. Equivalently, for every $\mathrm{C}$ and every $P \in \llbracket \mathrm{AC} \rrbracket_{+}$, one has $\left(\mathscr{X} \otimes \mathscr{I}_{\mathrm{C}}\right) P \in \llbracket \mathrm{BC} \rrbracket+$ for $\mathscr{X}=\mathscr{T}, \mathscr{C}-\mathscr{T}$. Let now $P \in \llbracket \mathrm{AC} \rrbracket$. Then one has

$$
\left(e_{\mathrm{BC}}\left|\left[(\mathscr{C}-\mathscr{T}) \otimes \mathscr{I}_{\mathrm{C}}\right]\right| P\right) \geq 0,
$$

and thus

$$
0 \leq\left(e_{\mathrm{BC}}\left|\left(\mathscr{T} \otimes \mathscr{I}_{\mathrm{C}}\right)\right| P\right) \leq\left(e_{\mathrm{AC}} \mid P\right) \leq 1 .
$$

This implies that $\left(\mathscr{T} \otimes \mathscr{I}_{\mathrm{C}}\right) P \in \llbracket \mathrm{AC} \rrbracket$. Finally, by the no-restriction hypothesis, $\mathscr{T} \in \llbracket \mathrm{A} \rightarrow \mathrm{B} \rrbracket$. The converse statement is trivial.

In the literature $[10,11]$ one can often find a different requirement under the name "no-restriction hypothesis", namely that for every system A the set $\llbracket \overline{\mathrm{A}} \rrbracket$ coincides with the set of functionals $a$ on $\llbracket \mathrm{A} \rrbracket_{\mathbb{R}}$ that satisfy $0 \leq(a \mid \rho) \leq 1$ for every $\rho \in \llbracket \mathrm{A} \rrbracket$. The last condition may be neither necessary nor sufficient for the no-restriction hypothesis as we state it, despite counterexamples are still unknown for both cases. Indeed, the no-restriction hypothesis for effects imposes the requirement that for every $\mathrm{C}$ and every state $P \in \llbracket \mathrm{AC} \rrbracket$, one has

$$
P \stackrel{\mathrm{C}}{\mathrm{C}} \in \llbracket \mathrm{C} \rrbracket .
$$

\subsection{Norms}

As the first step in the present work is to construct infinite composite systems, we will need a thorough notion of sequences and limits. From a topological point of view the vector spaces that we constructed so far have no special structure, 
however the operational procedures for discrimination of processes provide a natural definition of distance between events. Such a distance is related to the success probability in discriminating events. Making the space of events into a metric space, the operational distance immediately provides a topological structure through the induced operational norm. However, in order to make the space of events of infinite composite systems into a Banach algebra, a stronger norm is needed, that is introduced here: the sup-norm. In the quantum and classical case the two norms coincide.

The operational norm $\|\cdot\|_{\text {op }}$ for states is defined as follows [15].

Definition 5. The operational norm on $\llbracket \mathrm{A} \rrbracket_{\mathbb{R}}$ is

$$
\|\rho\|_{\mathrm{op}}:=\sup _{a \in \llbracket \overline{\mathrm{A}} \rrbracket}(2 a-e \mid \rho)=\sup _{\substack{a_{0}, a_{1} \in \llbracket \overline{\mathrm{A}} \rrbracket \\ a_{0}+a_{1}=e_{\mathrm{A}}}}\left(a_{0}-a_{1} \mid \rho\right) .
$$

The operational norm $\|\cdot\|_{\text {op }}$ for transformations $\mathscr{A} \in \llbracket \mathrm{A} \rightarrow \mathrm{B} \rrbracket_{\mathbb{R}}$ on finite systems is then defined as

$$
\begin{aligned}
\|\mathscr{A}\|_{\mathrm{op}} & :=\sup _{\mathrm{C}, \Psi \in \llbracket \mathrm{AC} \rrbracket_{1}}\left\|\left(\mathscr{A} \otimes \mathscr{I}_{\mathrm{C}}\right) \Psi\right\|_{\mathrm{op}} \\
& =\sup _{\mathrm{C}, \Psi \in \llbracket \mathrm{AC} \rrbracket} \sup _{a \in \llbracket \overline{\mathrm{B}} \overline{\mathrm{C}} \rrbracket}\left(2 a-e\left|\tilde{\mathscr{A}} \otimes \mathscr{I}_{\mathrm{C}}\right| \Psi\right) .
\end{aligned}
$$

In the special case of effects $a \in \llbracket \overline{\mathrm{A}} \rrbracket_{\mathbb{R}}$ we have

$$
\begin{aligned}
\|a\|_{\mathrm{op}} & :=\sup _{\mathrm{C}, \Psi \in \llbracket \mathrm{AC} \rrbracket}\left\|\left(a \otimes \mathscr{I}_{\mathrm{C}}\right) \Psi\right\|_{\mathrm{op}} \\
& =\sup _{\mathrm{C}, \Psi \in \llbracket \mathrm{AC} \rrbracket} \sup _{b \in \llbracket \overline{\mathrm{C}} \rrbracket}\left(a \otimes\left[2 b-e_{\mathrm{C}}\right] \mid \Psi\right) .
\end{aligned}
$$

As a consequence,

$$
\begin{aligned}
\|a\|_{\text {op }} & =\sup _{\substack{\rho_{0}, \rho_{1} \in \llbracket \mathrm{A} \rrbracket \\
\rho_{0}+\rho_{1} \in \llbracket \mathrm{A} \rrbracket}}\left(a \mid \rho_{0}-\rho_{1}\right) \\
& =\sup _{p \in[0,1]}\left\{p \sup _{\rho \in \llbracket \mathbb{A} \rrbracket}(a \mid \rho)-(1-p) \inf _{\sigma \in \llbracket \mathrm{A} \rrbracket}(a \mid \sigma)\right\} \\
& =\max \left\{\sup _{\rho \in \llbracket \mathrm{A} \rrbracket}(a \mid \rho), \inf _{\sigma \in \llbracket \mathrm{A} \rrbracket}(a \mid \sigma)\right\} \\
& =\sup _{\rho \in \llbracket \mathrm{A} \rrbracket}|(a \mid \rho)| .
\end{aligned}
$$

Here we only prove one new result about the operational norm, that we will use in the following.

Lemma 3. Let $\rho \in \llbracket \mathrm{A} \rrbracket_{\mathbb{R}}$ and $\sigma \in \llbracket \mathrm{B} \rrbracket_{1}$. Then

$$
\|\rho \otimes \sigma\|_{\mathrm{op}}=\|\rho\|_{\mathrm{op}} .
$$

Proof. By definition it is

$$
\begin{aligned}
\|\rho \otimes \sigma\|_{\text {op }} & =\sup _{\left(a_{0}, a_{1}\right)}\left(a_{0}-a_{1} \mid \rho \otimes \sigma\right) \\
& =\sup _{\left(b_{0}, b_{1}\right) \in M}\left(b_{0}-b_{1} \mid \rho\right),
\end{aligned}
$$

where $M:=\left\{\left(b_{0}, b_{1}\right) \mid b_{i}=a_{i}\left(\mathscr{I}_{\mathrm{A}} \otimes \sigma\right)\right\}$. Thus

$$
\|\rho \otimes \sigma\|_{\mathrm{op}} \leq\|\rho\|_{\mathrm{op}} .
$$

On the other hand, for every binary observationtest $\left(a_{0}, a_{1}\right)$ in $\llbracket \overline{\mathrm{A}} \rrbracket$ one has

$$
a_{i}=\left(a_{i} \otimes e_{\mathrm{B}}\right)\left(\mathscr{I}_{\mathrm{A}} \otimes \sigma\right),
$$

and thus $M$ actually contains every possible binary observation-test on A. Finally, this implies that

$$
\|\rho \otimes \sigma\|_{\mathrm{op}}=\|\rho\|_{\mathrm{op}} .
$$

For more details on $\|\cdot\|_{\text {op }}$ see Refs. $[8,15]$.

We now proceed to define the sup-norm.

Definition 6. The sup-norm $\|\mathscr{A}\|_{\text {sup }}$ of $\mathscr{A} \in$ $\llbracket \mathrm{A} \rightarrow \mathrm{B} \rrbracket_{\mathbb{R}}$ is defined as

$\|\mathscr{A}\|_{\text {sup }}:=\inf J(\mathscr{A})$,

$J(\mathscr{B}):=\left\{\lambda \mid \exists \mathscr{C} \in \llbracket \mathrm{A} \rightarrow \mathrm{B} \rrbracket_{1}, \lambda \mathscr{C} \succeq \mathscr{B} \succeq-\lambda \mathscr{C}\right\}$.

We now show that $\|\cdot\|_{\text {sup }}$ actually defines a norm.

Proposition 1. The sup-norm on $\llbracket \mathrm{A} \rightarrow \mathrm{B} \rrbracket_{\mathbb{R}}$ is well defined:

1. $\|\mathscr{A}\|_{\text {sup }}$ is non-negative, and it is null iff $\mathscr{A}=0$,

2. for $\mu \in \mathbb{R}$ one has $\|\mu \mathscr{A}\|_{\text {sup }}=|\mu|\|\mathscr{A}\|_{\text {sup }}$,

3. $\|\mathscr{A}+\mathscr{B}\|_{\text {sup }} \leq\|\mathscr{A}\|_{\text {sup }}+\|\mathscr{B}\|_{\text {sup }}$.

Proof. 1. Let $\mathscr{A} \in \llbracket \mathrm{A} \rightarrow \mathrm{B} \rrbracket_{\mathbb{R}}$. Suppose that $j:=\inf J(\mathscr{A})<0$. Then there exists $0<\varepsilon<|j|$ such that $j+\varepsilon \in J(\mathscr{A})$, namely

$$
\begin{aligned}
& -(|j|-\varepsilon) \mathscr{C} \succeq \mathscr{A} \succeq(|j|-\varepsilon) \mathscr{C} \\
& \Rightarrow-2(|j|-\varepsilon) \mathscr{C} \succeq 0,
\end{aligned}
$$

for some $\mathscr{C} \in \llbracket \mathrm{A} \rightarrow \mathrm{B} \rrbracket_{1}$, which is absurd. Then $\inf J(\mathscr{A}) \geq 0$. Suppose now that $J(\mathscr{A})=0$. This implies that for every $n \in \mathbb{N}$ there exists $\mathscr{C}_{n} \in \llbracket \mathrm{A} \rightarrow \mathrm{B} \rrbracket_{1}$ such that $\frac{1}{n} \mathscr{C}_{n} \succeq \mathscr{A} \succeq-\frac{1}{n} \mathscr{C}_{n}$. Now, by the closure of $\llbracket \mathrm{A} \rrbracket_{+}$in the operational norm, and considering that the sequences $\left(\frac{1}{n} \mathscr{C}_{n} \pm\right.$ 
$\mathscr{A})$ converge to $\pm \mathscr{A}$, it must be $\pm \mathscr{A} \in \llbracket \mathrm{A} \rrbracket_{+}$. This is possible if and only if $\mathscr{A}=0$. 2. The proof is trivial for $\mu=0$. Let then $\mu \neq 0$. If $x \in J(\mathscr{A})$, then $x \mathscr{C} \pm \mathscr{A} \succeq 0$ for some $\mathscr{C} \in$ $\llbracket \mathrm{A} \rightarrow \mathrm{B} \rrbracket_{1}$, and thus $|\mu|(x \mathscr{C} \pm \mathscr{A}) \succeq 0$, namely $|\mu| x \in J(\mu \mathscr{A})$. Thus $\|\mu \mathscr{A}\|_{\text {sup }} \leq|\mu|\|\mathscr{A}\|_{\text {sup }}$. For the same reason, $\|\mathscr{A}\|_{\text {sup }} \leq(1 /|\mu|)\|\mu \mathscr{A}\|_{\text {sup }}$, and finally $\|\mu \mathscr{A}\|_{\text {sup }}=|\mu|\|\mathscr{A}\|_{\text {sup }}$. 3. Let now $x \in$ $J(\mathscr{A})$ and $y \in J(\mathscr{B})$. Then $x \mathscr{C} \pm \mathscr{A} \succeq 0$, and $y \mathscr{D} \pm \mathscr{B} \succeq 0$, for $\mathscr{C}, \mathscr{D} \in \llbracket \mathrm{A} \rightarrow \mathrm{B} \rrbracket_{1}$. Thus $(x+$ y) $\mathscr{F} \pm(\mathscr{A}+\mathscr{B}) \succeq 0$, where $\mathscr{F}:=x /(x+y) \mathscr{C}+$ $y /(x+y) \mathscr{D} \in \llbracket \mathrm{A} \rightarrow \mathrm{B} \rrbracket_{1}$. Thus, $x+y \in J(\mathscr{A}+\mathscr{B})$, and then $\|\mathscr{A}+\mathscr{B}\|_{\text {sup }} \leq\|\mathscr{A}\|_{\text {sup }}+\|\mathscr{B}\|_{\text {sup }}$.

The following property makes $(\llbracket \mathrm{A} \rightarrow$ $\left.\mathrm{A} \rrbracket_{\mathbb{R}},\|\cdot\|_{\text {sup }}\right)$ a Banach algebra.

Proposition 2. For $\mathscr{A} \in \llbracket \mathrm{B} \rightarrow \mathrm{C} \rrbracket_{\mathbb{R}}$ and $\mathscr{B} \in$ $\llbracket \mathrm{A} \rightarrow \mathrm{B} \rrbracket_{\mathbb{R}},\|\mathscr{A} \mathscr{B}\|_{\text {sup }} \leq\|\mathscr{A}\|_{\text {sup }}\|\mathscr{B}\|_{\text {sup }}$.

Proof. Let $x \in J(\mathscr{A})$, and $y \in J(\mathscr{B})$. Then there are $\mathscr{C}, \mathscr{D}$ such that $x \mathscr{C} \pm \mathscr{A} \succeq 0, y \mathscr{D} \pm \mathscr{B} \succeq 0$. Now, we have

$$
\begin{aligned}
& \frac{1}{2}[(x \mathscr{C}+\mathscr{A})(y \mathscr{D}-\mathscr{B})+(x \mathscr{C}-\mathscr{A})(y \mathscr{D}+\mathscr{B}) \\
& =x y \mathscr{C} \mathscr{D}-\mathscr{A} \mathscr{B} \succeq 0 \\
& \frac{1}{2}[(x \mathscr{C}+\mathscr{A})(y \mathscr{D}+\mathscr{B})+(x \mathscr{C}-\mathscr{A})(y \mathscr{D}-\mathscr{B}) \\
= & x y \mathscr{C} \mathscr{D}+\mathscr{A} \mathscr{B} \succeq 0,
\end{aligned}
$$

and then $x y \in J(\mathscr{A} \mathscr{B})$. Thus, $\|\mathscr{A} \mathscr{B}\|_{\text {sup }} \leq$ $\|\mathscr{A}\|_{\text {sup }}\|\mathscr{B}\|_{\text {sup }}$.

Lemma 4. Let $\mathscr{A} \in \llbracket \mathrm{A} \rightarrow \mathrm{B} \rrbracket_{\mathbb{R}}$, and for an arbitrary $\mathrm{C}$, let $\mathscr{D} \in \llbracket \mathrm{C} \rightarrow \mathrm{D} \rrbracket_{1}$. Then $\|\mathscr{A} \otimes \mathscr{D}\|_{\text {sup }}=$ $\|\mathscr{A}\|_{\text {sup }}$.

Proof. Let $x \in J(\mathscr{A})$. Then by definition there exists $\mathscr{C} \in \llbracket \mathrm{A} \rightarrow \mathrm{B} \rrbracket_{1}$ such that $x \mathscr{C} \pm \mathscr{A} \succeq 0$. This implies that

$$
x \mathscr{C} \otimes \mathscr{D} \pm \mathscr{A} \otimes \mathscr{D} \succeq 0,
$$

namely $x \in J(\mathscr{A} \otimes \mathscr{D})$, and then $J(\mathscr{A}) \subseteq J(\mathscr{A} \otimes$ $\mathscr{D})$. Now, let $y \in J(\mathscr{A} \otimes \mathscr{D})$. Then there exists $\mathscr{C}^{\prime} \in \llbracket \mathrm{AC} \rightarrow \mathrm{BD} \rrbracket_{1}$ such that $y \mathscr{C}^{\prime} \pm \mathscr{A} \otimes \mathscr{D} \succeq 0$. Composing the 1.h.s. of the latter relation with $\psi \in \llbracket \mathrm{C} \rrbracket_{1}$ and $e_{\mathrm{D}}$, we obtain $y \mathscr{C}^{\prime \prime} \pm \mathscr{A} \succeq 0$, where $\mathscr{C}^{\prime \prime}:=\left(e_{\mathrm{D}}\left|\mathscr{C}^{\prime}\right| \psi\right)$ is a channel, and then $y \in J(\mathscr{A})$. Thus, $J(\mathscr{A} \otimes \mathscr{D}) \subseteq J(\mathscr{A})$. Finally, since $J(\mathscr{A} \otimes \mathscr{D})=J(\mathscr{A})$, we have $\|\mathscr{A} \otimes \mathscr{D}\|_{\text {sup }}=$ $\|\mathscr{A}\|_{\text {sup }}$.

Corollary 2. For $\mathscr{A} \in \llbracket \mathrm{A} \rightarrow \mathrm{B} \rrbracket_{\mathbb{R}}$, and for arbitrary $\mathrm{C}$, it is $\left\|\mathscr{A} \otimes \mathscr{I}_{\mathrm{C}}\right\|_{\text {sup }}=\|\mathscr{A}\|_{\text {sup }}$.
Corollary 3. For $\mathscr{A} \in \llbracket \mathrm{A} \rightarrow \mathrm{B} \rrbracket_{\mathbb{R}}$ and $\mathscr{B} \in$ $\llbracket \mathrm{C} \rightarrow \mathrm{D} \rrbracket_{\mathbb{R}},\|\mathscr{A} \otimes \mathscr{B}\|_{\text {sup }} \leq\|\mathscr{A}\|_{\text {sup }}\|\mathscr{B}\|_{\text {sup }}$

Proof. The result follows straightforwardly from proposition 2 and corollary 2 .

An important result regarding the sup-norm is provided by the following proposition.

Proposition 3. For $\mathscr{A} \in \llbracket \mathrm{B} \rightarrow \mathrm{C} \rrbracket_{\mathbb{R}}$ and $\mathscr{B} \in$ $\llbracket \mathrm{A} \rightarrow \mathrm{B} \rrbracket_{\mathbb{R}},\|\mathscr{A} \mathscr{B}\|_{\text {op }} \leq\|\mathscr{A}\|_{\text {sup }}\|\mathscr{B}\|_{\text {op }}$.

Proof. By definition we have

$$
\begin{aligned}
& \|\mathscr{A} \mathscr{B}\|_{\text {op }} \\
& =\sup _{\mathrm{D}, \Psi \in \llbracket \mathrm{AD} \rrbracket_{1}} \sup _{a \in \llbracket \overline{\mathrm{C}} \overline{\mathrm{D}} \rrbracket}\left(2 a-e_{\mathrm{CD}}\left|\mathscr{A} \mathscr{B} \otimes \mathscr{I}_{\mathrm{D}}\right| \Psi\right) .
\end{aligned}
$$

Now, for every $a \in \llbracket \overline{\mathrm{C}} \overline{\mathrm{D}} \rrbracket$, and $\lambda \in J(\mathscr{A})$, upon defining $a_{0}:=a, a_{1}:=e_{\mathrm{CD}}-a$, we have

$$
\begin{aligned}
& \left(a_{0}-a_{1} \mid \mathscr{A} \otimes \mathscr{I}_{\mathrm{C}}\right. \\
& =\left(a_{0} \mid\left[\mathscr{A} \otimes \mathscr{I}_{\mathrm{D}}\right]-\left(a_{1} \mid\left[\mathscr{A} \otimes \mathscr{I}_{\mathrm{D}}\right]\right.\right. \\
& =\lambda\left[\left(\tilde{a}_{0} \mid-\left(\tilde{a}_{1} \mid\right],\right.\right.
\end{aligned}
$$

where $\left(\tilde{a}_{i} \mid:=\lambda^{-1}\left\{\left(a_{i} \mid\left[\mathscr{A} \otimes \mathscr{I}_{\mathrm{D}}\right]+\frac{1}{2}(e \mid[(\lambda \mathscr{C}-\mathscr{A}) \otimes\right.\right.\right.$ $\left.\left.\mathscr{I}_{\mathrm{D}}\right]\right\}$, with $\mathscr{C}: \llbracket \mathrm{B} \rightarrow \mathrm{C} \rrbracket_{1}$ such that $\lambda \mathscr{C} \pm \mathscr{A} \succeq 0$. Clearly,

$$
\tilde{a}_{0}, \tilde{a}_{1} \in \llbracket \overline{\mathrm{B}} \overline{\mathrm{D}} \rrbracket, \quad \tilde{a}_{0}+\tilde{a}_{1}=e_{\mathrm{BD}},
$$

thus

$$
\begin{aligned}
& \left(a_{0}-a_{1}\left|\mathscr{A} \mathscr{B} \otimes \mathscr{I}_{\mathrm{D}}\right| \Psi\right) \\
& =\lambda\left[\left(\tilde{a}_{0}\left|-\left(\tilde{a}_{1} \mid\right] \mathscr{B} \otimes \mathscr{I}_{\mathrm{D}}\right| \Psi\right)\right. \\
& \leq \lambda \sup _{\bar{b} \overline{\mathrm{B}} \overline{\mathrm{D}}]}\left(2 b-e_{\mathrm{BD}}\left|\mathscr{B} \otimes \mathscr{I}_{\mathrm{D}}\right| \Psi\right)
\end{aligned}
$$

This implies that $\|\mathscr{A} \mathscr{B}\|_{\text {op }} \leq \lambda \sup _{b \in \llbracket \overline{\mathrm{B}} \overline{\mathrm{D}} \rrbracket}(2 b-$ $\left.e_{\mathrm{BD}}\left|\mathscr{B} \otimes \mathscr{I}_{\mathrm{D}}\right| \Psi\right)$, and then for every $\lambda \in J(\mathscr{A})$

$$
\begin{aligned}
& \|\mathscr{A} \mathscr{B}\|_{\text {op }} \\
& \leq \lambda \sup _{\mathrm{D}, \Psi \in \llbracket A D \rrbracket_{1}} \sup _{b \in \llbracket \overline{\mathrm{B}} \overline{\mathrm{D}} \rrbracket}\left(2 b-e_{\mathrm{BD}}\left|\mathscr{B} \otimes \mathscr{I}_{\mathrm{D}}\right| \Psi\right) \\
& =\lambda\|\mathscr{B}\|_{\text {op }} .
\end{aligned}
$$

Finally, taking the infimum over $\lambda \in J(\mathscr{A})$ we get

$$
\|\mathscr{A} \mathscr{B}\|_{\text {op }} \leq\|\mathscr{A}\|_{\text {sup }}\|\mathscr{B}\|_{\text {op }}
$$

Corollary 4. The sup-norm is stronger than the operational norm.

Proof. It is sufficient to observe that $\|\mathscr{A}\|_{\text {op }}=$ $\|\mathscr{A} \mathscr{I}\|_{\text {op }} \leq\|\mathscr{A}\|_{\text {sup }}\|\mathscr{I}\|_{\text {op }}=\|\mathscr{A}\|_{\text {sup }}$. 
Lemma 5. Let $\mathscr{A} \in \llbracket \mathrm{A} \rightarrow \mathrm{B} \rrbracket_{1}$ be a channel. Then $\|\mathscr{A}\|_{\text {sup }}=1$.

Proof. Clearly, $\mathscr{A} \succeq \mathscr{A} \succeq-\mathscr{A}$, thus $1 \in J(\mathscr{A})$, and $\inf J(\mathscr{A}) \leq 1$. On the other hand, suppose that there exists $1>\lambda \in J(\mathscr{A})$. This implies that there exists a channel $\mathscr{C} \in \llbracket \mathrm{A} \rightarrow \mathrm{B} \rrbracket_{1}$ such that $\mathscr{D}:=\lambda \mathscr{C}-\mathscr{A} \succeq 0$. However, this implies that $\left(\left.e\right|_{\mathrm{B}} \mathscr{D}=-(1-\lambda)\left(\left.e\right|_{\mathrm{A}} \succeq 0\right.\right.$. This is absurd, and then $\lambda \in J(\mathscr{A})$ must be $\lambda \geq 1$. Thus, $\inf J(\mathscr{A}) \geq 1$. Finally, this implies $\|\mathscr{A}\|_{\text {sup }}=$ 1.

Corollary 5. The sup-norm of the identity channel $\mathscr{I} \in \llbracket \mathrm{A} \rightarrow \mathrm{A} \rrbracket_{1}$ is 1 .

The sup-norm for effects is just the special case of the sup-norm of transformations with the output system equal to I.

Definition 7. Let $a \in \llbracket \overline{\mathrm{A}} \rrbracket_{\mathbb{R}}$, and let us define the half-line

$$
J(a):=\left\{\lambda \in \mathbb{R}_{+} \mid \lambda e_{\mathrm{A}} \succeq a \succeq-\lambda e_{\mathrm{A}}\right\} .
$$

The sup-norm $\|a\|_{\text {sup }}$ is defined as

$$
\|a\|_{\text {sup }}:=\inf J(a) .
$$

Proposition 4. The sup-norm on $\llbracket \overline{\mathrm{A}} \rrbracket_{\mathbb{R}}$ is well defined:

1. $\|a\|_{\text {sup }}$ is non-negative, and it is null iff $a=$ 0 ,

2. for $\mu \in \mathbb{R}$ one has $\|\mu a\|_{\text {sup }}=|\mu|\|a\|_{\text {sup }}$,

3. $\|a+b\|_{\text {sup }} \leq\|a\|_{\text {sup }}+\|b\|_{\text {sup }}$.

Proof. The case of effects is just a special case of the result of Proposition 1.

As an immediate consequence of proposition 3, we have the following result.

Corollary 6. The sup-norm is stronger than the operational norm on $\llbracket \bar{A} \rrbracket_{\mathbb{R}}$.

In the special case of $\llbracket \mathrm{I} \rightarrow \mathrm{I} \rrbracket_{\mathbb{R}}=\mathbb{R}$, one has $\llbracket \mathrm{I} \rightarrow \mathrm{I} \rrbracket_{1}=\{1\}$, and $\llbracket \mathrm{I} \rightarrow \mathrm{I} \rrbracket_{+}=\mathbb{R}_{+}$, thus $J(x)=$ $\{\lambda \in \mathbb{R} \mid \lambda \pm x \geq 0\}$. Clearly, $\|x\|_{\text {sup }}=\inf J(x)=$ $|x|$. We now need the following lemma.

Lemma 6. Let $a \in \llbracket \overline{\mathrm{A}} \rrbracket_{\mathbb{R}}$. Then $\left\|a \otimes e_{\mathrm{B}}\right\|_{\mathrm{op}}=$ $\|a\|_{\text {op }}$ and $\left\|a \otimes e_{\mathrm{B}}\right\|_{\text {sup }}=\|a\|_{\text {sup }}$.
Proof. For $\|\cdot\|_{\mathrm{op}}$, the equality trivially follows from the definition. For $\|\cdot\|_{\text {sup }}$, it is a special case of lemma 4 .

From lemma 5, the following consequence follows.

Corollary 7. For the deterministic effect $e_{\mathrm{A}}$ one has $\left\|e_{\mathrm{A}}\right\|_{\mathrm{sup}}=\left\|e_{\mathrm{A}}\right\|_{\mathrm{op}}=1$.

We now prove a result that will be very useful later.

Lemma 7. Let $\mathscr{A} \in \llbracket \mathrm{A} \rightarrow \mathrm{B} \rrbracket_{+}$, and $(a \mid:=$ $\left(e_{\mathrm{B}} \mid \mathscr{A} \in \llbracket \overline{\mathrm{A}} \rrbracket_{+}\right.$. Then $\|\mathscr{A}\|_{\text {sup }}=\|a\|_{\text {sup }}$.

Proof. First of all, by proposition 2, one has $\|a\|_{\text {sup }} \leq\left\|e_{\mathrm{A}}\right\|_{\text {sup }}\|\mathscr{A}\|_{\text {sup }}=\|\mathscr{A}\|_{\text {sup }}$. On the other hand, let $\lambda \in J(a)$. This implies that

$$
b:=\lambda e_{\mathrm{A}}-a \succeq 0 .
$$

Let us then define $\mathscr{B}:=\mid \rho)\left(b \mid \in \llbracket \mathrm{A} \rightarrow \mathrm{B} \rrbracket_{+}\right.$, for some $\rho \in \llbracket \mathrm{B} \rrbracket_{1}$. By theorem 3, it is easy to check that $\mathscr{A}+\mathscr{B}=\lambda \mathscr{C}$ for $\mathscr{C} \in \llbracket \mathrm{A} \rightarrow \mathrm{B} \rrbracket_{1}$. Then,

$$
\lambda \mathscr{C}-\mathscr{A}=\mathscr{B} \succeq 0,
$$

which means that $\lambda \in J(\mathscr{A})$. Then, $J(a) \subseteq$ $J(\mathscr{A})$, and finally $\|\mathscr{A}\|_{\text {sup }} \leq\|a\|_{\text {sup }}$.

In the case of Classical and Quantum Theory, phrasing the definitions of sup- and operational norm in terms of semidefinite programming problems, one can conclude that they are strongly dual. As a consequence, in these special cases they define the same norm.

\section{The quasi-local algebra in OPTs}

Following the definition of Ref. [31], we define a cellular automaton in a general OPT as a triple $(G, \mathrm{~A}, \mathscr{V})$, where $G$ is a denumerable set of labels for the systems that compose the automaton - addresses of the memory cells-, $\mathrm{A}_{G}$ is a (possibly infinite) composite system corresponding to the collection of systems $\mathrm{A}_{g}$ labelled by elements $g \in G$-i.e. the memory array-, and $\mathscr{V}$ is a reversible transformation on $\llbracket \overline{\mathrm{A}}_{G} \rrbracket$, such that $\mathscr{V} \cdot \mathscr{V}^{-1}$ is an automorphism of $\llbracket \mathrm{A}_{G} \rightarrow \mathrm{A}_{G} \rrbracket$. However, this is definition is incomplete, for many reasons. In the first place, most of the objects mentioned above are not thoroughly defined. The purpose of the present section is to set the ground for the rigorous definition of a cellular automaton. 
We start fixing some notation. For every $R \subseteq$ $G$ let

$$
\mathrm{A}_{R}:=\bigotimes_{g \in R} \mathrm{~A}_{g}
$$

with the convention that $\mathrm{A}_{\emptyset}:=\mathrm{I}$. The full system is then $\mathrm{A}_{G}=\bigotimes_{g \in G} \mathrm{~A}_{g}$. This purely formal notion will now be thoroughly substantiated.

With a slight abuse of notation, we will often use $R=g$ instead of $R=\{g\}$, dropping the braces. The set of finite regions of $G$ will be denoted as

$$
\mathrm{R}^{(G)}:=\{R \subseteq G|| R \mid<\infty\},
$$

while the set of arbitrary regions of $G$ will be denoted as

$$
\overline{\mathrm{R}}^{(G)}:=\{R \subseteq G\} .
$$

Clearly $\mathrm{R}^{(G)} \subseteq \overline{\mathrm{R}}^{(G)}$. In the remainder, when we write e.g. $e_{R}$ or $\mathscr{I}_{R}$ for $R \in \mathrm{R}^{(G)}$, we mean the deterministic effect or the identity transformation for the system $\mathrm{A}_{R}$, respectively.

The following construction of $\otimes_{g \in G} \mathrm{~A}_{g}$ is inspired by that of Refs. [49, 50], however with significant differences.

\subsection{Quasi-local effects}

In this subsection we start the mathematical construction of the system $\mathrm{A}_{G}$, the parallel composition of infinitely many finite systems. The first object that we will define is the space of generalised effects, along with its convex cone of positive effects, and the convex set of effects. The construction is based on the notion of a local effect, that is an effect which acts non trivially only on finitely many systems labelled by $g \in R$, with $R \in \mathrm{R}^{(G)}$. We will say that such an effect acts on the finite region $R$. We will then introduce a real vector space structure over the set of local effects, and a norm that is induced by the sup norm for local effects. The last step is then the topological closure of the space of local effects in the sup-norm. The Banach space thus obtained is the space of generalised quasi-local effects, and the positive cone along with the convex set of effects contain the limits of sequences of elements of local cones or convex sets of effects, respectively.

As we will see in the next section, the definition of the space of quasi-local states is much more involved than that of quasi-local effects. The latter is particularly simple, thanks to causality, that provides us with a preferential local effect, the unique deterministic one, without the need of introducing any arbitrary choice of a reference local effect. Moreover, while the construction of the space of quasi-local states is not logically necessary, as we will find them as a subspace of bounded functionals on quasi-local effects, the same is not true of effects.

If we defined effects as the dual space of quasilocal states, we would end up with far more effects than needed, while lacking sectors of the state space, that are usually reached by the evolution of a quasi-local state through a cellular automaton. These are the main reasons why our construction begins with effects.

The first notion we introduce is that of a local effect. Let $\mathrm{A}_{G}$ denote the formal composition of countably many systems from a general OPT: $\mathrm{A}_{G}:=\bigotimes_{g \in G} \mathrm{~A}_{g}$. Intuitively speaking, a local effect of $\mathrm{A}_{G}$ is an event within a test that discards all the systems in $G$ but the finite region $R$, where a non-trivial measurement is performed. We then define the local effect $(a, R)$ as a pair made of an effect $a \in \llbracket \overline{\mathrm{A}}_{R} \rrbracket$ and the region $R$. To lighten the notation, in the remainder of the paper we will use the symbol $a_{R}$ instead of $(a, R)$. The set of local effects is denoted by

$$
\begin{aligned}
& \text { Pre } \llbracket \overline{\mathrm{A}}_{G} \rrbracket_{L} \\
& :=\bigsqcup_{R \in \mathrm{R}^{(G)}} \llbracket \overline{\mathrm{A}}_{R} \rrbracket=\left\{a_{R} \mid R \in \mathrm{R}^{(G)}, a \in \llbracket \overline{\mathrm{A}}_{R} \rrbracket\right\} .
\end{aligned}
$$

The above definition can be widened encompassing generalised effects:

$$
\operatorname{Pre} \llbracket \overline{\mathrm{A}}_{G} \rrbracket_{L \mathbb{R}}:=\bigsqcup_{R \in \mathrm{R}^{(G)}} \llbracket \overline{\mathrm{A}}_{R} \rrbracket \mathbb{R} .
$$

Let us now consider a partition of the region $R$ into two disjoint regions $S_{0} \cup S_{1}=R$. Since in the definition of a local effect $a_{R}$ we did not set constraints on the effect $a \in \llbracket \overline{\mathrm{A}}_{R} \rrbracket_{\mathbb{R}}$, it might be that $a=e_{S_{0}} \otimes a^{\prime}$, with $a^{\prime} \in \llbracket \overline{\mathrm{A}}_{S_{1}} \rrbracket_{\mathbb{R}}$. It is clear from our intuitive notion of a local effect that $a_{R}$ and $a_{S_{1}}^{\prime}$ should represent the same effect. This observation leads us to the equivalence relation defined as follows.

Definition 8 (Equivalent local effects). We say that the effects $a_{R}$ and $a_{S}^{\prime}$ in Pre $\llbracket \overline{\mathrm{A}}_{G} \rrbracket_{L \mathbb{R}}$ are equivalent, and denote this as $a_{R} \sim a_{S}^{\prime}$, if there 
exists $a_{0} \in \llbracket \overline{\mathrm{A}}_{R \cap S} \rrbracket_{\mathbb{R}}$ such that the following identities hold

$$
\left\{\begin{array}{l}
a=a_{0} \otimes e_{S \backslash R}, \\
a^{\prime}=a_{0} \otimes e_{R \backslash S} .
\end{array}\right.
$$

It is clear that the real notion of a local effect is captured by the equivalence classes modulo the above equivalence relation. We thus quotient $\operatorname{Pre} \llbracket \overline{\mathrm{A}}_{G} \rrbracket_{\mathbb{R}}$ and define the obtained set as the set of local effects of $\mathrm{A}_{G}$.

Definition 9. A generalised local effect is an equivalence class $\left[a_{R}\right]_{\sim}$. The set of generalised local effects of $\mathrm{A}_{G}$ is

$$
\llbracket \overline{\mathrm{A}}_{G} \rrbracket_{L \mathbb{R}}:=\operatorname{Pre} \llbracket \overline{\mathrm{A}}_{G} \rrbracket_{L \mathbb{R}} / \sim .
$$

With a slight abuse of notation, in the following we will write $a_{R}$ instead of $\left[a_{R}\right]_{\sim}$, unless the context requires explicit distinction of the two symbols. One can easily prove the following result

Lemma 8. Let $a_{R} \in \operatorname{Pre} \llbracket \overline{\mathrm{A}}_{G} \rrbracket_{L \mathbb{R}}$. Then for every finite region $H \in \mathrm{R}^{(G)}$ such that $H \cap R=\emptyset$, one has $\left(a \otimes e_{H}\right)_{R \cup H} \in \operatorname{Pre} \llbracket \overline{\mathrm{A}}_{G} \rrbracket_{L \mathbb{R}}$, and $(a \otimes$ $\left.e_{H}\right)_{R \cup H} \sim a_{R}$.

The proof of the above lemma is straightforward, and we do not report it here.

Let us now come back to our initial goal, that is to define a local effect as an event that acts non-trivially only on a finite region $R$. Intuition leads again to figure out what is the preferred representative of the class of a local effect: within the equivalence class, it is the element defined on the smallest region. We now provide a formal definition of such a minimal representative, and show that it is well posed.

Definition 10. The minimal representative of the equivalence class $a_{R}$, denoted as $\tilde{a}_{R_{a}}$, is defined through

$$
R_{a}:=\bigcap_{S \in \mathrm{R}_{(a, R)}} S, \quad \tilde{a}_{R_{a}} \sim a_{R}
$$

where $\mathrm{R}_{(a, R)}$ is the set of all those finite regions $S \in \mathrm{R}^{(G)}$ for which there exists $b \in \llbracket \overline{\mathrm{A}}_{S} \rrbracket_{\mathbb{R}}$ such that $b_{S} \sim a_{R}$.

Lemma 9. The minimal representative exists and is unique.
Proof. As to existence, we remark that, by Eq. (11), $R_{a}$ is the set of all $g \in G$ such that for all regions $S \in \mathrm{R}_{(a, R)}$ one has $g \in S$. Thus, if $h \notin R_{a}$, there must exist $S \in \mathrm{R}_{(a, R)}$ such that $h \notin S$. This implies that i) by definition there exists $f_{S} \sim a_{R}$, and ii) by lemma $8 c_{S \cup h} \sim a_{R}$, with

$$
c=f \otimes e_{h} .
$$

As a consequence, if $c_{T} \sim a_{R}$ and $h \in T$, but $h \notin R_{a}$, by definition (10) $c_{T}$ must be of the form of Eq. (12), for some $f \in \llbracket \overline{\mathrm{A}}_{T \backslash h} \rrbracket_{\mathbb{R}}$. Clearly, $R_{a} \in \mathrm{R}^{(G)}$, since for any $S \in R_{(a, R)}$ one has $R_{a} \subseteq$ $S$. Now, let $S \in \mathrm{R}_{(a, R)}$, and $c_{S} \sim a_{R}$. One has $S=R_{a} \cup S^{\prime}$, where $S^{\prime}:=\left(S \backslash R_{a}\right) \in \mathrm{R}^{(G)}$, thus $R_{a} \cap S^{\prime}=\emptyset$. By Eq. (12), we then have

$$
c=b \otimes e_{S^{\prime}}, \quad b \in \llbracket \overline{\mathrm{A}}_{R_{a}} \rrbracket_{\mathbb{R}} .
$$

We now define $\tilde{a}_{R_{a}}:=b_{R_{a}}$, and finally, since Eq. 13 holds for any $c_{S} \sim a_{R}$, one can easily verify that $\tilde{a}_{R_{a}} \sim a_{R}$.

As to uniqueness, we remark that the region $R_{a}$ is uniquely defined. Now, suppose that there were two different $b, c \in \llbracket \overline{\mathrm{A}}_{R_{a}} \rrbracket_{\mathbb{R}}$ such that $b_{R_{a}}, c_{R_{a}} \sim a_{R}$. Then by Eq. (10) it must be $b=c$.

We now make local effects into a vector space.

Definition 11. Let $a_{R}, b_{S} \in \llbracket \overline{\mathrm{A}}_{G} \rrbracket_{L \mathbb{R}}$, and $h \in \mathbb{R}$. Then we define

$$
\begin{aligned}
& h a_{R}:= \begin{cases}(h a)_{R} & h \neq 0, \\
0_{\emptyset} & h=0,\end{cases} \\
& a_{R}+b_{S}:=c_{R \cup S} \\
& c:=a \otimes e_{S \backslash R}+b \otimes e_{R \backslash S} .
\end{aligned}
$$

Notice that it is not always true that $R_{c}=R_{a} \cup$ $R_{b}$. As an example, consider $a=f_{g_{1}} \otimes f_{g_{2}}$ and $b=$ $f_{g_{1}} \otimes\left(e-f_{g_{2}}\right)$, with $f_{g_{1}} \neq e_{g_{1}}, 0 \neq f_{g_{2}} \neq e_{g_{2}}$, and $R_{a}=R_{b}=\left\{g_{1}, g_{2}\right\}$. Then $c=a+b=f_{g_{1}} \otimes e_{g_{2}}$, and clearly $R_{c}=\left\{g_{1}\right\}$, which is strictly included in $R_{a}=R_{b}=R_{a} \cup R_{b}$.

It is easy to check that $\llbracket \overline{\mathrm{A}}_{G} \rrbracket_{L \mathbb{R}}$ is a real vector space with null element given by the equivalence class of $0_{\emptyset}$.

We now equip the real vector space of local effects with a norm, and we then close it to obtain the Banach space of quasi-local effects. The definition is based on a norm for effects of finite 
systems, as every local effect $a_{R} \in \llbracket \overline{\mathrm{A}}_{G} \rrbracket_{L \mathbb{R}}$ reduces to the effect $\tilde{a} \in \llbracket \overline{\mathrm{A}}_{R_{a}} \rrbracket_{\mathbb{R}}$. A natural norm one might think of is then the operational norm, that induces the following definition.

Definition 12. The operational norm $\left\|a_{R}\right\|_{\text {op }}$ of $a_{R} \in \llbracket \overline{\mathrm{A}}_{G} \rrbracket_{L \mathbb{R}}$ is defined by the following expression

$$
\left\|a_{R}\right\|_{\mathrm{op}}:=\|\tilde{a}\|_{\mathrm{op}},
$$

where $\tilde{a} \in \llbracket \overline{\mathrm{A}}_{R_{a}} \rrbracket_{\mathbb{R}}$.

Unfortunately, if one completes the space of local effects in the operational norm, in general the dual norm on the space of bounded linear functionals - which will be our state space - does not coincide with the operational norm on the state space, for those states that can be interpreted as quasi-local preparations. We will then choose a different norm on our space of effects. The new norm will be referred to as sup-norm, as it is the extension of the sup-norm to the infinite case.

As far as finite-dimensional systems are concerned, this choice does not represent a problem, as all norms are equivalent in finite-dimensional vector spaces. However, the sup-norm is stronger than the operational one - see corollary 4-, and thus the space that we construct, completing our normed vector space of local effects with supnorm Cauchy sequences, might contain distinct limits that are operationally equivalent. This point is a delicate one, and to avoid an unreasonable construction where there exist different effects that are operationally equivalent, we impose a sufficient constraint for the operational norm and the sup-norm to be equivalent also for infinite systems: we restrict attention to those theories where the following property holds: there exists a finite constant $k$ such that for every system A and every $a \in \llbracket \overline{\mathrm{A}} \rrbracket_{\mathbb{R}}$

$$
\|a\|_{\text {sup }} \leq k\|a\|_{\text {op }} .
$$

This implies that not only the bound (15) holds for a fixed system A, but it holds with a fixed constant independent of the system A. In turn, a sufficient condition for (15) is that for every system A

$$
\llbracket \mathrm{A} \rrbracket_{+}^{*} \equiv \llbracket \overline{\mathrm{A}} \rrbracket_{+},
$$

namely every positive functional on the cone of states is proportional to an effect by a positive constant, and viceversa. In all the presently known theories the latter condition is satisfied. The two above conditions in Eqs. (15) and (16) are discussed in detail in Appendix A, where we prove that condition (16) implies condition (15).

Our new norm is an order-unit norm. As we will discuss in subsection 4.2, its dual coincides with the operational norm on quasi-local states.

Let us now see the definition of the sup-norm in detail.

Definition 13. The sup-norm $\left\|a_{R}\right\|_{\text {sup }}$ of $a_{R} \in$ $\llbracket \overline{\mathrm{A}}_{G} \rrbracket_{L \mathbb{R}}$ is defined by the following expression

$$
\left\|a_{R}\right\|_{\text {sup }}:=\|\tilde{a}\|_{\text {sup }}
$$

where $\tilde{a} \in \llbracket \overline{\mathrm{A}}_{R_{a}} \rrbracket \mathbb{R}$.

We now want to prove that the operational norm and the sup-norm are well-defined norms on $\llbracket \overline{\mathrm{A}}_{G} \rrbracket_{L \mathbb{R}}$.

Proposition 5. Let $a_{R} \sim \tilde{a}_{R_{a}}$. Then $\left\|a_{R}\right\|_{\text {op }}=$ $\|a\|_{\mathrm{op}}$, and $\left\|a_{R}\right\|_{\mathrm{sup}}=\|a\|_{\mathrm{sup}}$.

Proof. Let $a_{R} \sim \tilde{a}_{R_{a}}$, and $R=R_{a} \cup R^{\prime}$, with $R_{a} \cap R^{\prime}=\emptyset$. Then by Eq. (10) we have

$$
a=\tilde{a} \otimes e_{R^{\prime}},
$$

and thus by definitions 12 and 13 and lemma 6 , it is

$$
\begin{aligned}
& \left\|a_{R}\right\|_{\mathrm{op}}=\|\tilde{a}\|_{\mathrm{op}}=\left\|\tilde{a} \otimes e_{R^{\prime}}\right\|_{\mathrm{op}}=\|a\|_{\mathrm{op}}, \\
& \left\|a_{R}\right\|_{\mathrm{sup}}=\|\tilde{a}\|_{\mathrm{sup}}=\left\|\tilde{a} \otimes e_{R^{\prime}}\right\|_{\mathrm{sup}}=\|a\|_{\mathrm{sup}} .
\end{aligned}
$$

We can then prove the desired result.

Proposition 6. The functionals $\|\cdot\|_{\mathrm{op}}$ and $\|\cdot\|_{\mathrm{sup}}$ are norms on $\llbracket \overline{\mathrm{A}}_{G} \rrbracket_{L \mathbb{R}}$.

Proof. 1. For every $a_{R} \in \llbracket \overline{\mathrm{A}}_{G} \rrbracket_{L \mathbb{R}}$ it is clear that $\left\|a_{R}\right\|_{\text {op }} \geq 0$ and $\left\|a_{R}\right\|_{\text {sup }} \geq 0$. Now, $\|\tilde{a}\|_{\text {op }}=$ $\|\tilde{a}\|_{\text {sup }}=0$ if and only if $\tilde{a}=0$, namely, reminding definition 11, $a_{R}=0_{\emptyset}$. 2. For every $a_{R} \in \llbracket \overline{\mathrm{A}}_{G} \rrbracket_{L \mathbb{R}}$, by definitions 7,11 , and 12 , one straightforwardly has that, for every $\mu \in \mathbb{R}$,

$$
\begin{aligned}
& \left\|\mu a_{R}\right\|_{\mathrm{op}}=\|\mu \tilde{a}\|_{\mathrm{op}}=|\mu|\|\tilde{a}\|_{\mathrm{op}}=|\mu|\left\|a_{R}\right\|_{\mathrm{op}} \\
& \left\|\mu a_{R}\right\|_{\mathrm{sup}}=\|\mu \tilde{a}\|_{\mathrm{sup}}=|\mu|\|\tilde{a}\|_{\mathrm{sup}}=|\mu|\left\|a_{R}\right\|_{\mathrm{sup}} .
\end{aligned}
$$

3. Let now $c_{R \cup S}=a \otimes e_{S \backslash R}+e_{R \backslash S} \otimes b$ for $a \in \llbracket \overline{\mathrm{A}}_{R} \rrbracket_{\mathbb{R}}$ and $b \in \llbracket \overline{\mathrm{A}}_{S} \rrbracket_{\mathbb{R}}$, as from definition 11 . 
Then, by lemma 6 , proposition 5 and by the triangle inequality, we have

$$
\begin{aligned}
& \left\|a_{R}+b_{S}\right\|_{\mathrm{op}}=\left\|c_{R \cup S}\right\|_{\mathrm{op}}=\|c\|_{\mathrm{op}} \\
& \quad \leq\|a\|_{\mathrm{op}}+\|b\|_{\mathrm{op}}=\left\|a_{R}\right\|_{\mathrm{op}}+\left\|b_{S}\right\|_{\mathrm{op}} \\
& \left\|a_{R}+b_{S}\right\|_{\mathrm{sup}}=\left\|c_{R \cup S}\right\|_{\mathrm{sup}}=\|c\|_{\mathrm{sup}} \\
& \leq\|a\|_{\text {sup }}+\|b\|_{\text {sup }}=\left\|a_{R}\right\|_{\mathrm{sup}}+\left\|b_{S}\right\|_{\mathrm{sup}} .
\end{aligned}
$$

We remark that, in a theory that satisfies assumption (16), one has $\|\cdot\|_{\text {op }} \equiv\|\cdot\|_{\text {sup }}$ on $\llbracket \bar{A} \rrbracket_{\mathbb{R}}$, and thus $\|\cdot\|_{\text {op }}=\|\cdot\|_{\text {sup }}$ on $\llbracket \overline{\mathrm{A}}_{G} \rrbracket_{\mathbb{R}}$ (the proof can be found in appendix A). In general, however, the sup-norm is stronger than the operational norm, as we now prove.

Lemma 10. Let $a_{R} \in \llbracket \overline{\mathrm{A}}_{G} \rrbracket_{L \mathbb{R}}$. Then $\left\|a_{R}\right\|_{\text {op }} \leq$ $\left\|a_{R}\right\|_{\text {sup }}$.

Proof. Let us consider $\mu \in J(a)$. Then for every $\rho \in \llbracket \mathrm{A}_{R} \rrbracket_{1}$ we have

$$
\left(\mu e_{R} \pm a \mid \rho\right) \geq 0,
$$

i.e. $|(a \mid \rho)| \leq \mu$. Then, taking the supremum over states on l.h.s. we obtain $\left\|a_{R}\right\|_{\text {op }} \leq \mu$, and finally, taking the infimum over $J(a)$ we obtain the thesis.

The space of local effects that we constructed so far is a normed real vector space. We now make it into a Banach space, the space of quasilocal effects, by the usual completion procedure for normed spaces. We first introduce the space $\llbracket \overline{\mathrm{A}}_{G} \rrbracket_{C \mathbb{R}}$ of Cauchy sequences

$$
a: \mathbb{N} \rightarrow \llbracket \overline{\mathrm{A}}_{G} \rrbracket_{L \mathbb{R}}:: n \mapsto a_{n R_{n}} .
$$

We then define the equivalence relation between Cauchy sequences in $\llbracket \overline{\mathrm{A}}_{G} \rrbracket_{C \mathbb{R}}$ defined by $a \cong b$ iff $\lim _{n \rightarrow \infty}\left\|a_{n R_{n}}-b_{n S_{n}}\right\|_{\text {sup }}=0$. Finally, we define the space $\llbracket \overline{\mathrm{A}}_{G} \rrbracket_{Q \mathbb{R}}$ of generalised quasi-local effects, by taking the quotient

$$
\llbracket \overline{\mathrm{A}}_{G} \rrbracket_{Q \mathbb{R}}:=\llbracket \overline{\mathrm{A}}_{G} \rrbracket_{C \mathbb{R}} / \cong .
$$

The elements of this space will be denoted by $a=\left[a_{n R_{n}}\right]$. In this context, the class of a constant Cauchy sequence $\left(R_{n}=R\right.$ and $\left.a_{n R_{n}}=a_{R}\right)$ will be denoted by $a_{R}:=\left[a_{n R_{n}}\right]$. Clearly, since $\left|\left\|a_{n R_{n}}\right\|_{\text {sup }}-\left\|a_{m R_{m}}\right\|_{\text {sup }}\right| \leq\left\|a_{n R_{n}}-a_{m R_{m}}\right\|_{\text {sup }}$, for a Cauchy sequence $a=\left[a_{n R_{n}}\right]$ also $\left\|a_{n R_{n}}\right\|_{\text {sup }}$ is a Cauchy sequence, and we define $\|a\|_{\text {sup }}:=$ $\lim _{n \rightarrow \infty}\left\|a_{n R_{n}}\right\|_{\text {sup }}$. One can easily verify that the sup-norm on $\llbracket \overline{\mathrm{A}}_{G} \rrbracket_{Q \mathbb{R}}$ thus defined is independent of the specific sequence within an equivalence class. The space $\llbracket \overline{\mathrm{A}}_{G} \rrbracket_{Q \mathbb{R}}$ is by construction a real Banach space, and $\llbracket \overline{\mathrm{A}}_{G} \rrbracket_{L \mathbb{R}}$ can be identified with the dense submanifold containing constant sequences $a_{R}=\left[a_{R}\right]$. Clearly, in this case $\|a\|_{\text {sup }}=\left\|a_{R}\right\|_{\text {sup }}$.

Definition 14. Let $a \in \llbracket \overline{\mathrm{A}}_{G} \rrbracket_{Q \mathbb{R}}$. If there is a Cauchy sequence $a_{n R_{n}}$ in the class defining $a$, such that, for some $n_{0} \in \mathbb{N}$, for every $n \geq n_{0}$ one has $\tilde{a}_{n} \in \llbracket \overline{\mathrm{A}}_{R_{n}} \rrbracket$, then we call a a quasi-local effect. We denote the set of quasi-local effects by $\llbracket \overline{\mathrm{A}}_{G} \rrbracket_{Q}$.

The cone $\llbracket \overline{\mathrm{A}}_{G} \rrbracket_{Q+}$ contains all elements that are proportional to an element in $\llbracket \overline{\mathrm{A}}_{G} \rrbracket_{Q}$ by a positive constant.

The deterministic quasi-local effect $e_{G}$ is the class $e_{G}:=1_{\emptyset}$.

The cone $\llbracket \overline{\mathrm{A}}_{G} \rrbracket_{Q+}$ introduces a partial ordering in $\llbracket \overline{\mathrm{A}}_{G} \rrbracket_{Q \mathbb{R}}$, that we denote by

$$
a \succeq b \Leftrightarrow a-b \in \llbracket \overline{\mathrm{A}}_{G} \rrbracket_{Q+} .
$$

Quasi-local effects can be interpreted as effects that can be arbitrarily well approximated by local observation procedures. The effect $e_{G}$ plays an important role, as it is the unique deterministic effect in $\llbracket \overline{\mathrm{A}}_{G} \rrbracket_{Q}$. This will be proved shortly. Let us start providing the first important property of $e_{G}$.

Lemma 11. Let $a \in \llbracket \overline{\mathrm{A}}_{G} \rrbracket_{Q}$. Then also $e_{G}-a \in$ $\llbracket \overline{\mathrm{A}}_{G} \rrbracket_{Q}$.

Proof. Let $a=\left[a_{n R_{n}}\right]$. Let $a_{n R_{n}}^{\prime}:=\left(e-a_{n}\right)_{R_{n}} \in$ $\llbracket \overline{\mathrm{A}}_{R_{n}} \rrbracket$. Then one has $\left(a_{n}^{\prime}-a_{m}^{\prime}\right)_{R_{n} \cup R_{m}}=\left(a_{m}-\right.$ $\left.a_{n}\right)_{R_{m} \cup R_{n}}$, and thus $a_{n R_{n}}^{\prime}$ is a Cauchy sequence whose class we call $a^{\prime} \in \llbracket \overline{\mathrm{A}}_{G} \rrbracket_{Q}$. Finally, since $a+a^{\prime}$ is the class $\left[a_{n R_{n}}\right]+\left[a_{n R_{n}}^{\prime}\right]=\left[\left(a_{n}+a_{n}^{\prime}\right)_{R_{n}}\right]=$ $\left[1_{\emptyset}\right]=e_{G}$, we have that $a+a^{\prime}=e_{G}$, namely $e_{G}-a=a^{\prime} \in \llbracket \overline{\mathrm{A}}_{G} \rrbracket_{Q}$.

By the above lemma we know that not only $e_{G} \succeq a$ for every quasi-local effect $a$, but also that every quasi-local effect $a$ can be complemented by a quasi-local effect $a^{\prime}$ such that $a+a^{\prime}=e_{G}$. In other words $\left(a, e_{G}-a\right)$ is a binary quasi-local observation test.

We can also prove the converse result.

Lemma 12. Let $a \in \llbracket \overline{\mathrm{A}}_{G} \rrbracket_{Q+}$, and $e_{G}-a \in$ $\llbracket \overline{\mathrm{A}}_{G} \rrbracket_{Q+}$. Then $a, e_{G}-a \in \llbracket \overline{\mathrm{A}}_{G} \rrbracket_{Q}$. 
Proof. Indeed, the statement is true for finite systems thanks to the no-restriction hypothesis (see theorem 5), and thus it holds for a given Cauchy sequence in the class of $a$, namely if $a_{n R_{n}}^{\prime}:=\left(e-a_{n}\right)_{R_{n}} \in \llbracket \overline{\mathrm{A}}_{R_{n}} \rrbracket_{+}$for all $n$, then $a_{n}, a_{n}^{\prime} \in \llbracket \overline{\mathrm{A}}_{R_{n}} \rrbracket$. Thus, $a=\lim _{n \rightarrow \infty} a_{n} \in \llbracket \overline{\mathrm{A}}_{G} \rrbracket_{Q}$, and $a^{\prime}=\lim _{n \rightarrow \infty} a_{n}^{\prime}=e_{G}-a \in \llbracket \overline{\mathrm{A}}_{G} \rrbracket_{Q}$.

We now prove that $e_{G}$ lies in the interior of $\llbracket \overline{\mathrm{A}}_{G} \rrbracket_{Q+}$, namely there is a ball of radius $r$ around $e_{G}$ in sup-norm that is contained in $\llbracket \overline{\mathrm{A}}_{G} \rrbracket_{Q+}$.

Lemma 13. There exists an open ball $B_{r}\left(e_{G}\right)$ of radius $r$ in $\llbracket \overline{\mathrm{A}}_{G} \rrbracket_{Q \mathbb{R}}$ that is fully contained in $\llbracket \overline{\mathrm{A}}_{G} \rrbracket_{Q+}$.

Proof. Let $\left\|a-e_{G}\right\|_{\text {sup }}<r<1 / 2$. Then, by definition, there exist a sequence $a_{n R_{n}}$ such that $\lim _{n \rightarrow \infty} a_{n}=a$, and $n_{0} \in \mathbb{N}$ such that for $n \geq n_{0}$

$\left\|a_{n}-e_{G}\right\|_{\text {sup }} \leq\left\|a_{n}-a\right\|_{\text {sup }}+\left\|a-e_{G}\right\|_{\text {sup }}<2 r$.

This implies that

$$
\left[2 r e+\left(a_{n}-e\right)\right]_{R_{n}}=\left[a_{n}-(1-2 r) e\right]_{R_{n}} \succeq 0 .
$$

Now, this implies that for $n \geq n_{0}$ it is $a_{n} \succeq$ $(1-2 r) e_{R_{n}} \succeq 0$, and thus $a_{n} \in \llbracket \overline{\mathrm{A}}_{G} \rrbracket_{L+}$. Finally, by definition, one obtains $a \in \llbracket \overline{\mathrm{A}}_{G} \rrbracket_{Q+}$. Thus, the ball $B_{r}\left(e_{G}\right)$ is contained in $\llbracket \overline{\mathrm{A}}_{G} \rrbracket_{Q+}$.

Corollary 8. Let $a \in \llbracket \overline{\mathrm{A}}_{G} \rrbracket_{Q+}$. Then for $\varepsilon>0$, $B_{\varepsilon r}\left(\varepsilon e_{G}+a\right) \subseteq \llbracket \overline{\mathrm{A}}_{G} \rrbracket_{Q+}$ for some $r>0$.

Proof. Let $b \in B_{\varepsilon r}\left(\varepsilon e_{G}+a\right)$. Then

$$
\begin{aligned}
& \left\|b-a-\varepsilon e_{G}\right\|_{\text {sup }}<\varepsilon r \\
& \Leftrightarrow\left\|(b-a) / \varepsilon-e_{G}\right\|_{\text {sup }}<r .
\end{aligned}
$$

Then $(b-a) / \varepsilon=c \in \llbracket \overline{\mathrm{A}}_{G} \rrbracket_{Q+}$, ad thus

$$
b=\varepsilon c+a \in \llbracket \overline{\mathrm{A}}_{G} \rrbracket_{Q+} .
$$

We now prove that the deterministic effect $e_{G}$ allows for an extension of the defining property of the sup-norm.

Lemma 14. The sup-norm of $a \in \llbracket \overline{\mathrm{A}}_{G} \rrbracket_{Q \mathbb{R}}$ can be expressed as

$$
\begin{aligned}
& \|a\|_{\text {sup }}=\inf J(a), \\
& J(a):=\left\{\mu \geq 0 \mid \mu e_{G} \succeq a \succeq-\mu e_{G}\right\} .
\end{aligned}
$$

Proof. In the case of local effects $a=a_{R}$ the statement is a trivial recasting of the definition of sup-norm. Let us then consider the class $a \in$ $\llbracket \overline{\mathrm{A}}_{G} \rrbracket_{Q \mathbb{R}}$ with $a=\left[a_{n R_{n}}\right]$. One has, by definition, $\|a\|_{\text {sup }}=\lim _{n \rightarrow \infty}\left\|a_{n}\right\|_{\text {sup }}$. Let us consider the sequence $\mu_{n}:=\left\|a_{n}\right\|_{\text {sup }}+\varepsilon \in J\left(a_{n}\right)$. Clearly, $\lim _{n \rightarrow \infty} \mu_{n}=\|a\|_{\text {sup }}+\varepsilon$. Moreover, since

$$
\begin{aligned}
& \left\|\left\{\left(\mu_{n}-\mu_{m}\right) e \pm\left(a_{n}-a_{m}\right)\right\}_{R_{n} \cup R_{m}}\right\|_{\text {sup }} \\
& \quad \leq\left|\left\|a_{m}\right\|_{\text {sup }}-\left\|a_{n}\right\|_{\text {sup }}\right|+\left\|a_{m}-a_{n}\right\|_{\text {sup }} \leq 2 \varepsilon,
\end{aligned}
$$

the sequence $\left\{\left(\mu_{n}+\varepsilon\right) e \pm a_{n}\right\}_{R_{n}}$ converges to $\left(\|a\|_{\text {sup }}+\varepsilon\right) e_{G} \pm a \in \llbracket \overline{\mathrm{A}}_{G} \rrbracket_{Q+}$, thus for every $\varepsilon \geq 0$ one has $\|a\|_{\text {sup }}+\varepsilon \in J(a)$. This implies that

$$
\inf J(a) \leq\|a\|_{\text {sup }} .
$$

On the other hand, let $\mu \in J(a)$, then

$$
\mu e_{G} \pm a \succeq 0 .
$$

By corollary 8 , we have that, for $\varepsilon>0$,

$$
(\varepsilon+\mu) e_{G} \pm a \in B_{\varepsilon r}\left(\varepsilon e_{G}+\mu e_{G} \pm a\right) \subseteq \llbracket \overline{\mathrm{A}}_{G} \rrbracket_{Q+} .
$$

Now, this implies that there are two open balls, centred at $(\mu+\varepsilon) e_{G} \pm a$, that are fully contained in $\llbracket \overline{\mathrm{A}}_{G} \rrbracket_{Q+}$. There exists then $n_{0} \in \mathbb{N}$ such that for $n \geq n_{0}$ one has

$$
(\mu+\varepsilon) e_{G} \pm a_{n} \succeq 0
$$

namely $(\mu+\varepsilon) \in J\left(a_{n}\right)$. Thus, $J(a) \subseteq J\left(a_{n}\right)-\varepsilon$, and

$$
\inf J(a) \geq\left\|a_{n}\right\|_{\text {sup }}-\varepsilon
$$

Finally, this implies that inf $J(a) \geq\|a\|_{\text {sup }}$.

As the cone $\llbracket \overline{\mathrm{A}}_{G} \rrbracket_{Q+}$ is closed, we can easily prove that the infimum in the expression of the sup norm is actually a minimum.

Lemma 15. Let $a \in \llbracket \overline{\mathrm{A}}_{G} \rrbracket_{Q \mathbb{R}}$. Then

$$
\|a\|_{\text {sup }} e_{G} \pm a \succeq 0 \text {. }
$$

Proof. By lemma 14, for every $\varepsilon>0$ one has

$$
\left(\|a\|_{\text {sup }}+\varepsilon\right) e_{G} \pm a \succeq 0 .
$$

The sequences $b_{n}^{ \pm}:=\left(\|a\|_{\text {sup }}+1 / n\right) e_{G} \pm a$ are both Cauchy, and their limits are both in $\llbracket \overline{\mathrm{A}}_{G} \rrbracket_{Q+}$, then

$$
\|a\|_{\text {sup }} e_{G} \pm a \succeq 0
$$


Before concluding, we remark that the operational norm can be extended to $\llbracket \overline{\mathrm{A}}_{G} \rrbracket_{Q \mathbb{R}}$, by simply defining for $a=\left[a_{n R_{n}}\right]$

$$
\|a\|_{\mathrm{op}}:=\lim _{n \rightarrow \infty}\left\|a_{n R_{n}}\right\|_{\mathrm{op}} .
$$

Indeed, since

$$
\begin{aligned}
\left|\left\|a_{n R_{n}}\right\|_{\mathrm{op}}-\left\|a_{m R_{m}}\right\|_{\mathrm{op}}\right| & \leq\left\|a_{n R_{n}}-a_{m R_{m}}\right\|_{\mathrm{op}} \\
& \leq\left\|a_{n R_{n}}-a_{m R_{m}}\right\|_{\mathrm{sup}},
\end{aligned}
$$

the sequence $\left\|a_{n R_{n}}\right\|_{\text {op }}$ is Cauchy, and the limit is well defined. Moreover, also on $\llbracket \overline{\mathrm{A}}_{G} \rrbracket_{Q \mathbb{R}}$ the sup-norm is stronger than the operational norm, as can be straightforwardly concluded from lemma 10. However, in theories where there exists $k>0$ such that $\|\cdot\|_{\text {sup }} \leq k\|\cdot\|_{\text {op }}$ in $\llbracket \overline{\mathrm{A}} \rrbracket_{\mathbb{R}}$ for every finite system $\mathrm{A}$, the same inequality holds in the limit, and the operational and sup-norm are equivalent. In particular, this is the case under assumption (16).

We now introduce a diagrammatic notation for effects in $\llbracket \overline{\mathrm{A}} \rrbracket_{Q \mathbb{R}}$ that will make part of the subsequent proofs and arguments more intuitive. First of all, we denote a quasi-local effect $a \in \llbracket \overline{\mathrm{A}}_{G} \rrbracket_{Q \mathbb{R}}$ by the symbol

$$
\stackrel{G}{a} .
$$

In the case of a local effect $a_{R} \in \llbracket \overline{\mathrm{A}}_{G} \rrbracket_{L \mathbb{R}}$, we will draw

$$
G a_{R}=\frac{R}{G \backslash R}, e .
$$

Notice that, since for $\left(a \otimes e_{R_{1}}\right)_{R}$ with $a \in \llbracket \overline{\mathrm{A}}_{R_{0}} \rrbracket$ and $R_{1}:=R \backslash R_{0}$, it is $\left(a \otimes e_{R_{1}}\right)_{R} \sim a_{R_{0}}$, we have

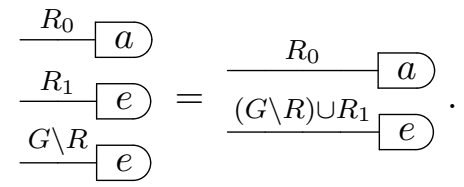

From the above identity, we can intuitively conclude that

$$
\stackrel{(G \backslash R) \cup R_{1}}{e}=\frac{R_{1} e}{G \backslash R} .
$$

Indeed, let $G=R \cup H$, with $|R|<\infty$. Then $\left[e_{R}\right]=\left[1_{\emptyset}\right]=e_{G}$, which is the equation represented by the diagram in Eq. 24. Similarly, let $a_{R} \otimes b_{H}:=\lim _{n \rightarrow \infty}\left(a \otimes b_{n}\right)$, where $\left[b_{n S_{n}}\right]=b \in$ $\llbracket \overline{\mathrm{A}}_{H} \rrbracket_{Q \mathbb{R}}$. By corollary 3 , one has $\left[\left(a \otimes b_{n}\right)_{R \cup S_{n}}\right]=$ $\left(a_{R} \otimes b_{H}\right)$. Thus, in $G=R \cup H$, one has
$a_{R}=\left[a_{R}\right]=\left[\left(a_{R} \otimes e_{H}\right)\right]=a_{R} \otimes e_{H}$, which is the meaning of Eq. (22).

As a final remark, we observe that for every denumerable set $G$, and every subset $R \subseteq G$, including infinite ones, one can define $\llbracket \overline{\mathrm{A}}_{R}^{(\bar{G})} \rrbracket_{Q \mathbb{R}}$ as the closed subspace spanned by those quasi-local effects $a=a_{n R_{n}}$ such that, for every $n \in \mathbb{N}, R_{n} \subseteq$ $R$. If one constructs the system $\mathrm{A}_{R}:=\bigotimes_{g \in R} \mathrm{~A}_{g}$, it is straightforward to construct an ordered Banach space isomorphism

$$
\mathscr{J}_{R}^{\dagger}: \llbracket \overline{\mathrm{A}}_{R}^{(G)} \rrbracket_{Q \mathbb{R}} \rightarrow \llbracket \overline{\mathrm{A}}_{R} \rrbracket_{Q \mathbb{R}}::\left[a_{n R_{n}}\right]_{G} \mapsto\left[a_{n R_{n}}\right]_{R},
$$

with the norm coinciding with the sup-norm in both spaces. The left-inverse of $\mathscr{J}_{R}^{\dagger}$ is

$$
\mathscr{J}_{R}^{-1 \dagger}: \llbracket \overline{\mathrm{A}}_{R} \rrbracket_{Q \mathbb{R}} \rightarrow \llbracket \overline{\mathrm{A}}_{R}^{(G)} \rrbracket_{Q \mathbb{R}}
$$

$\mathscr{J}_{R}^{\dagger}$ and $\mathscr{J}_{R}^{-1 \dagger}$ can be diagrammatically denoted as

$$
\begin{aligned}
& \stackrel{R}{\mathscr{J}_{R}^{\dagger}} \underset{G \backslash R}{R}=\frac{R}{a}=(a, \\
& \mathscr{J}_{R}^{-1 \dagger} R \quad a=\frac{R}{G \backslash R}, a
\end{aligned}
$$

\subsection{Extended states}

Now that we defined the space of quasi-local effects, we can define the space of states as the space of bounded linear functionals on $\llbracket \bar{A}_{G} \rrbracket_{Q \mathbb{R}}$. The set of states is then defined considering those linear functionals that, acting on local effects of an arbitrary finite region $R$, behave as a state in $\llbracket \mathrm{A}_{R} \rrbracket$.

Definition 15 (Generalised extended states). The space $\llbracket \mathrm{A}_{G} \rrbracket_{\mathbb{R}}$ of generalised extended states of $\mathrm{A}_{G}$ is the topological dual of $\llbracket \overline{\mathrm{A}}_{G} \rrbracket_{Q \mathbb{R}}$, i.e. the Banach space $\llbracket \overline{\mathrm{A}}_{G} \rrbracket_{Q \mathbb{R}}^{*}$ of bounded linear functionals on $\llbracket \overline{\mathrm{A}}_{G} \rrbracket_{Q \mathbb{R}}$, equipped with the norm

$$
\|\rho\|_{*}:=\sup _{\|a\|_{\text {sup }}=1}|(a \mid \rho)| .
$$

The operational norm on $\llbracket \mathrm{A}_{G} \rrbracket_{\mathbb{R}}$, defined as

$$
\|\rho\|_{\text {op }}:=\sup _{a \in \llbracket \overline{\mathrm{A}}_{G} \rrbracket_{Q}}\left(2 a-e_{G} \mid \rho\right),
$$

coincides with the norm $\|\cdot\|_{*}$, as we now prove. 
Lemma 16. Let $\rho \in \llbracket \mathrm{A}_{G} \rrbracket \mathbb{R}$. Then

$$
\|\rho\|_{*}=\sup _{a \in \llbracket \overline{\mathrm{A}}_{G} \rrbracket_{Q}}\left(2 a-e_{G} \mid \rho\right) .
$$

Proof. Let $0 \leq \varepsilon \leq\|\rho\|_{\text {op }}$. We then have

$$
0 \leq\|\rho\|_{\text {op }}-\varepsilon \leq\left(2 a-e_{G} \mid \rho\right),
$$

for some $a \in \llbracket \overline{\mathrm{A}}_{G} \rrbracket_{Q}$. Invoking lemma 11, one has

$$
\begin{aligned}
& e_{G}+\left(2 a-e_{G}\right)=2 a \succeq 0, \\
& e_{G}-\left(2 a-e_{G}\right)=2\left(e_{G}-a\right) \succeq 0,
\end{aligned}
$$

and, by lemma $14,\left\|2 a-e_{G}\right\|_{\text {sup }} \leq 1$. Then it is

$$
\|\rho\|_{\text {op }}-\varepsilon \leq \frac{\left(2 a-e_{G} \mid \rho\right)}{\left\|2 a-e_{G}\right\|_{\text {sup }}} \leq\|\rho\|_{*} .
$$

On the other hand, let us now pick $a \in \llbracket \overline{\mathrm{A}}_{G} \rrbracket_{\mathbb{R}}$ with $\|a\|_{\text {sup }}=1$. Then, by lemma $15, e_{G} \pm a \succeq 0$. If we define $a_{ \pm}:=\frac{1}{2}\left(e_{G} \pm a\right)$, we have $a_{ \pm} \succeq 0$, and

$$
a_{+}+a_{-}=e_{G}, \quad\left(a_{+}-a_{-}\right)=a,
$$

which by lemma 12 implies that $a_{ \pm} \in \llbracket \overline{\mathrm{A}}_{G} \rrbracket_{Q}$. Then

$$
\pm(a \mid \rho)=\left(2 a_{ \pm}-e_{G} \mid \rho\right) .
$$

Thus, $|(a \mid \rho)| \leq\|\rho\|_{\text {op }}$, for every $\varepsilon>0$, and taking the supremum on the l.h.s. we obtain $\|\rho\|_{*} \leq$ $\|\rho\|_{\text {op }}$.

Technically speaking, the two norms $\|\cdot\|_{\text {sup }}$ and $\|\cdot\|_{*}$ are a base and order-unit norm pair [51].

What is missing now is the notion of a convex set of proper states, the extended preparations of our infinite system $\mathrm{A}_{G}$. Let us then give the following definition.

Definition 16 (Local restriction). Given a state $\rho \in \llbracket \mathrm{A}_{G} \rrbracket_{\mathbb{R}}$, the local restriction of $\rho$ to $S \in \mathrm{R}^{(G)}$ is the functional $\rho_{\mid S} \in \llbracket \mathrm{A}_{S} \rrbracket_{\mathbb{R}}$ defined through

$$
\left(a \mid \rho_{\mid S}\right):=\left(a_{S} \mid \rho\right), \quad \forall a \in \llbracket \overline{\mathrm{A}}_{S} \rrbracket .
$$

The notion of a restriction can be brought further, considering infinite regions, by invoking the isomorphism $\mathscr{J}_{R}$ defined in Eq. (25), as follows.

Definition 17 (Restriction). Given a state $\rho \in$ $\llbracket \mathrm{A}_{G} \rrbracket_{\mathbb{R}}$, the restriction of $\rho$ to $S \in \overline{\mathrm{R}}^{(G)}$ is the functional $\rho_{\mid S} \in \llbracket \mathrm{A}_{S} \rrbracket_{\mathbb{R}}$ defined through

$$
\left(a \mid \rho_{\mid S}\right):=\left(\mathscr{J}_{S}^{-1 \dagger} a \mid \rho\right), \quad \forall a \in \llbracket \overline{\mathrm{A}}_{S} \rrbracket .
$$

Defining $\hat{\mathscr{J}}_{S}^{-1}$ by duality as

$$
\left(a \mid \hat{\mathscr{J}}_{S}^{-1} \rho\right):=\left(\mathscr{J}_{S}^{-1 \dagger} a \mid \rho\right),
$$

we can then equivalently express Eq. (34) as

$$
\rho_{\mid S}=\hat{\mathscr{J}}_{S}^{-1} \rho .
$$

Representing generalised extended states through diagrams, and reminding eq. (28), the above equations can be recast as

$$
\begin{aligned}
\rho_{\mid S} S & =\rho, \mathscr{J}_{S}^{-1} S a \\
& =\rho \frac{S \backslash S}{a},
\end{aligned}
$$

which can be taken as the definition of the equation

$$
\rho_{\mid S} S^{S}:=\rho_{G \backslash S}^{S} \in
$$

We can now introduce the set of states $\llbracket \mathrm{A}_{G} \rrbracket$ as the special set of generalised extended states whose restrictions are all states. In other words, an element $\rho$ of the space $\llbracket \mathrm{A}_{G} \rrbracket \mathbb{R}$ is a state if, restricted to any finite region $R \in \mathrm{R}^{(G)}$, it defines a state for that region.

Definition 18 (Extended states). The set $\llbracket \mathrm{A}_{G} \rrbracket$ of states in the space of generalised extended states $\llbracket \mathrm{A}_{G} \rrbracket_{\mathbb{R}}$ is the set of those elements $\rho \in$ $\llbracket \mathrm{A}_{G} \rrbracket_{\mathbb{R}}$ such that for every $R \in \mathrm{R}^{(G)}$ the local restriction of $\rho$ to $R$ is a state $\rho_{\mid R} \in \llbracket \mathrm{A}_{R} \rrbracket$. Deterministic states, whose set is denoted by $\llbracket \mathrm{A}_{G} \rrbracket_{1}$, are those states $\rho \in \llbracket \mathrm{A}_{G} \rrbracket$ with $\left(e_{G} \mid \rho\right)=1$.

One can easily verify that the set $\llbracket \mathrm{A}_{G} \rrbracket$ is convex, as a consequence of convexity of $\llbracket \mathrm{A} \rrbracket$ for every finite system A. As we did for finite systems, we also define a positive cone generated by $\llbracket \mathrm{A}_{G} \rrbracket$. Moreover, it is easy to check that the restriciton of a state to an infinite region $S \in \overline{\mathrm{R}}^{(G)}$ is s state in $\llbracket \mathrm{A}_{S} \rrbracket$.

Definition 19 (Extended positive cone). The set $\llbracket \mathrm{A}_{G} \rrbracket+$ in the space of generalised extended states $\llbracket \mathrm{A}_{G} \rrbracket_{\mathbb{R}}$ is the cone of those elements $\rho \in \llbracket \mathrm{A}_{G} \rrbracket_{\mathbb{R}}$ such that there exist $\bar{\rho} \in \llbracket \mathrm{A}_{G} \rrbracket$ and $\mu \geq 0$ such that $\rho=\mu \bar{\rho}$.

We can now show an important result about the restriction of states. 
Lemma 17. The restriction $\hat{\mathscr{J}}_{R}^{-1} \llbracket \mathrm{A}_{G} \rrbracket$ of the set of states of $\mathrm{A}_{G}$ coincides with $\llbracket \mathrm{A}_{R} \rrbracket$.

Proof. It is a straightforward exercise to verify that $\hat{\mathscr{J}}_{R}^{-1} \llbracket \mathrm{A}_{G} \rrbracket \subseteq \llbracket \mathrm{A}_{R} \rrbracket$. For the converse, let $\rho \in \llbracket \mathrm{A}_{R} \rrbracket$, and let us now construct a state $\sigma \in$ $\llbracket \mathrm{A}_{G} \rrbracket$ such that $\sigma_{\mid R}=\rho$. We define $\bar{R}:=G \backslash R$. There are two possible situations: $\bar{R} \in \mathrm{R}^{(G)}$, or $\bar{R} \in \overline{\mathrm{R}}^{(G)}$. If $\bar{R} \in \mathrm{R}^{(G)}$, let $a_{T} \in \llbracket \overline{\mathrm{A}}_{G} \rrbracket L \mathbb{R}$, with $T \in \mathrm{R}^{(G)}$. We define

$$
(a \mid \sigma):=\left(a \mid \rho_{\mid T \cap R} \otimes \nu_{\mid T \cap \bar{R}}\right),
$$

for an arbitrary $\nu \in \llbracket \mathrm{A}_{\bar{R}} \rrbracket$, where we introduced the notation $\sigma_{\mid S^{\prime}}$ for $S^{\prime} \subseteq S \in \mathrm{R}^{(G)}$, and $\sigma$ a state of the composite system $\sigma \in \llbracket \mathrm{A}_{S} \rrbracket=\llbracket \mathrm{A}_{S^{\prime}} \mathrm{A}_{S \backslash S^{\prime}} \rrbracket$, with

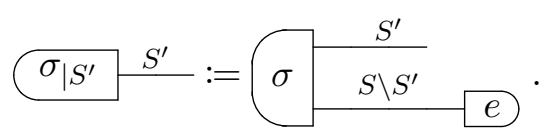

Clearly, the functional $\sigma$ is bounded, and thus it can be extended to a functional on the full space $\llbracket \overline{\mathrm{A}}_{G} \rrbracket_{Q \mathbb{R}}$, i.e. $\sigma \in \llbracket \mathrm{A}_{G} \rrbracket_{\mathbb{R}}$. Moreover one can easily verify that $\sigma_{\mid T} \in \llbracket \mathrm{A}_{T} \rrbracket$ for every $T \in \mathrm{R}^{(G)}$, thus $\sigma$ is the desired state in $\llbracket \mathrm{A}_{G} \rrbracket$. A similar construction can be carried out for the case $\bar{R} \in$ $\overline{\mathrm{R}}^{(G)}$, taking $\nu \in \llbracket \mathrm{A}_{\bar{R}} \rrbracket$. By construction, also in this case $\sigma \in \llbracket \mathrm{A}_{G} \rrbracket$, as can be straightforwardly checked.

The next result that we prove is that quasilocal effects $a \in \llbracket \overline{\mathrm{A}}_{G} \rrbracket_{Q}$ are positive on the set $\llbracket \mathrm{A}_{G} \rrbracket$.

Lemma 18. Let $a \in \llbracket \overline{\mathrm{A}}_{G} \rrbracket_{Q}$, and $\rho \in \llbracket \mathrm{A}_{G} \rrbracket$. Then $(a \mid \rho) \geq 0$

Proof. Let $a \in \llbracket \overline{\mathrm{A}}_{G} \rrbracket_{Q}$ be a local effect $a_{R}=$ $\left[a_{R}\right]$. Then, by definition, $\left(a_{R} \mid \rho\right)=\left(a \mid \rho_{\mid R}\right) \geq$ 0 . Now, let $a=\left[a_{n R_{n}}\right]$. We have $(a \mid \rho)=$ $\lim _{n \rightarrow \infty}\left(a_{n R_{n}} \mid \rho\right) \geq 0$.

The following result draws a first analogy between the properties of the sup-norm for finite systems and that for infinite parallel compositions.

Lemma 19. Let $\rho \in \llbracket \mathrm{A}_{G} \rrbracket_{+}$. Then $\|\rho\|_{*}=$ $\left(e_{G} \mid \rho\right)$.

Proof. Let $\rho \in \llbracket \mathrm{A}_{G} \rrbracket_{+}$. Then, by virtue of lemma $18,(a \mid \rho) \geq 0$ for all $a \in \llbracket \overline{\mathrm{A}}_{G} \rrbracket$. Let now $\|a\|_{\text {sup }}=1$. By lemma 15 , one has $e_{G} \pm a \succeq 0$, and thus $\left(e_{G} \mid \rho\right) \geq|(a \mid \rho)|$. Then $\left(e_{G} \mid \rho\right) \geq\|\rho\|_{*}$.
On the other hand, being $e_{G}$ a legitimate element of $\llbracket \overline{\mathrm{A}}_{G} \rrbracket_{Q}$ with $\left\|e_{G}\right\|_{\text {sup }}=1$, one has $\|\rho\|_{*}=\sup _{\|a\|_{\text {sup }}=1}|(a \mid \rho)| \geq\left(e_{G} \mid \rho\right)$.

Thanks to lemma 12, we have the following corollary.

Corollary 9. Let $\rho \in \llbracket \mathrm{A}_{G} \rrbracket$. Then $0 \leq(a \mid \rho) \leq$ $\left(e_{G} \mid \rho\right)$ for every $a \in \llbracket \overline{\mathrm{A}}_{G} \rrbracket_{Q}$.

We can now show that also in the infinite case every state is proportional to a deterministic one. Let us first prove a preliminary lemma.

Lemma 20. Let $\rho \in \llbracket \mathrm{A}_{G} \rrbracket \mathbb{R}$. If $(a \mid \rho)=0$ for every $a \in \llbracket \overline{\mathrm{A}}_{G} \rrbracket_{Q}$, then $\rho=0$.

Proof. If $(a \mid \rho)=0$ for every $a \in \llbracket \overline{\mathrm{A}}_{G} \rrbracket_{Q}$ then, using lemma 11, we have

$\left(2 a-e_{G} \mid \rho\right)=(a \mid \rho)-\left(e_{G}-a \mid \rho\right)=0, \quad \forall a \in \llbracket \overline{\mathrm{A}}_{G} \rrbracket_{Q}$.

This implies that $\|\rho\|_{*}=0$, and thus $\rho=0$.

Proposition 7. Every state $\rho \in \llbracket \mathrm{A}_{G} \rrbracket$ is proportional to a deterministic state $\bar{\rho} \in \llbracket \mathrm{A}_{G} \rrbracket_{1}$.

Proof. Let $\rho \in \llbracket \mathrm{A}_{G} \rrbracket$. If $\rho=0$, then for every $\bar{\rho} \in \llbracket \mathrm{A}_{G} \rrbracket_{1}$ it is $\rho=0 \bar{\rho}$. Let then $\rho \neq 0$. By lemmas 18 and 20, there must exist $a \in \llbracket \overline{\mathrm{A}}_{G} \rrbracket$ such that $(a \mid \rho)>0$. By corollary 9, one then has $\left(e_{G} \mid \rho\right) \geq(a \mid \rho)>0$. By definition, for every $S \in \mathrm{R}^{(G)}$, we have

$$
\left(e_{\mathrm{A}_{S}} \mid \rho_{\mid S}\right)=\left(e_{S} \mid \rho\right)=\left(e_{G} \mid \rho\right)>0 .
$$

Thus, if we set $\bar{\rho}:=\rho /\left(e_{G} \mid \rho\right)$, for every $S \in \mathrm{R}^{(G)}$ we obtain

$$
\left(e_{\mathrm{A}_{S}} \mid \bar{\rho}_{\mid S}\right)=\left(e_{G} \mid \bar{\rho}\right)=1,
$$

implying that $\bar{\rho}_{\mid S} \in \llbracket \mathrm{A}_{S} \rrbracket_{1}$ for every $S \in \mathrm{R}^{(G)}$. Then, $\bar{\rho} \in \llbracket \mathrm{A}_{G} \rrbracket_{1}$, and $\bar{\rho}$ is such that $\rho=\left(e_{G} \mid \rho\right) \bar{\rho}$.

We now show that the operational norm is equivalently defined on $\llbracket \overline{\mathrm{A}}_{G} \rrbracket_{Q \mathbb{R}}$ by

$$
\|a\|_{\text {op }}:=\sup _{\rho \in \llbracket \mathrm{A}_{G} \rrbracket}|(a \mid \rho)| .
$$

Indeed, let $a=\left[a_{n R_{n}}\right]$. Then

$$
|(a \mid \rho)|=\lim _{n \rightarrow \infty}\left|\left(a_{n R_{n}} \mid \rho\right)\right|=\lim _{n \rightarrow \infty}\left|\left(a_{n} \mid \rho_{\mid R_{n}}\right)\right| .
$$

This implies that

$$
|(a \mid \rho)| \leq\left|\left(a_{n} \mid \rho_{\mid R_{n}}\right)\right|+\varepsilon \leq\left\|a_{n R_{n}}\right\|_{\text {op }}+\varepsilon,
$$


thus $\sup _{\rho \in \llbracket \mathrm{A}_{G} \rrbracket}|(a \mid \rho)| \leq\|a\|_{\text {op }}$. On the other hand, for every $a_{n R_{n}}$ and every $\varepsilon$, there exists $\rho_{\varepsilon} \in \llbracket \mathrm{A}_{R_{n}} \rrbracket$ such that $\left\|a_{n R_{n}}\right\|_{\text {op }} \leq\left|\left(a_{n} \mid \rho_{\varepsilon}\right)\right|+\varepsilon$. Now, by lemma 17, this implies that for every $\varepsilon$ there exists $\tilde{\rho}_{\varepsilon} \in \llbracket \mathrm{A}_{G} \rrbracket$ such that

$$
\left\|a_{n R_{n}}\right\|_{\mathrm{op}}-\varepsilon \leq\left|\left(a_{n R_{n}} \mid \tilde{\rho}_{\varepsilon}\right)\right| .
$$

Finally, this implies that for every $\varepsilon$ there exists $\rho_{\varepsilon} \in \llbracket \mathrm{A}_{G} \rrbracket$ such that

$$
\begin{aligned}
\|a\|_{\mathrm{op}}-3 \varepsilon & \leq\left\|a_{n R_{n}}\right\|_{\mathrm{op}}-2 \varepsilon \\
& \leq\left|\left(a_{n R_{n}} \mid \tilde{\rho}_{\varepsilon}\right)\right|-\varepsilon \\
& \leq\left|\left(a \mid \tilde{\rho}_{\varepsilon}\right)\right| .
\end{aligned}
$$

Thus, in conclusion, $\|a\|_{\text {op }} \leq \sup _{\rho \in \llbracket \mathrm{A}_{G} \rrbracket}|(a \mid \rho)|$.

A crucial property of $\llbracket \mathrm{A}_{G} \rrbracket$ is that extended states are separating for $\llbracket \overline{\mathrm{A}}_{G} \rrbracket_{Q}$, namely if for every state two generalised effects give the same value, then they coincide.

Theorem 6 (States separate effects). Let $a \in$ $\llbracket \overline{\mathrm{A}}_{G} \rrbracket_{Q \mathbb{R}}$. If $(a \mid \rho)=0$ for all $\rho \in \llbracket \mathrm{A}_{G} \rrbracket_{1}$, then $a=0$.

Proof. Let $a=\left[a_{n R_{n}}\right] \in \llbracket \overline{\mathrm{A}}_{G} \rrbracket_{Q \mathbb{R}}$, and $(a \mid \rho)=0$ for every $\rho \in \llbracket \mathrm{A}_{G} \rrbracket_{1}$. Then for every $\rho \in \llbracket \mathrm{A}_{G} \rrbracket_{1}$ one has

$$
\left|\left(a_{n} \mid \rho\right)\right|=\left|\left(a_{n}-a \mid \rho\right)\right| \leq\left\|a_{n}-a\right\|_{\text {sup }},
$$

and thus for every $\varepsilon>0$ there exists $n_{0}$ such that, for $n \geq n_{0},\left\|a_{n}\right\|_{\text {op }} \leq \varepsilon$. Thus, we have $\|a\|_{\text {op }}=0$. As a consequence, for every $\varepsilon>0$ there exists $n_{0}$ such that for $n \geq n_{0}$ one has

$$
\left\|a_{n}\right\|_{\text {sup }} \leq k\left\|a_{n}\right\|_{\text {op }} \leq \varepsilon,
$$

and thus $\|a\|_{\text {sup }}=0$. Now, this implies that $a=$ 0 .

Remark 2. The above result is made possible by requirement (15) that the sup-norm and the operational norm for effects of finite systems A are bounded as

$$
\forall a \in \llbracket \overline{\mathrm{A}} \rrbracket_{\mathbb{R}}\|a\|_{\text {sup }} \leq k\|a\|_{\mathrm{op}},
$$

with $k$ independent of the system $\mathrm{A}$. In particular, this is true under the assumption (16), i.e. that $\llbracket \overline{\mathrm{A}} \rrbracket_{+}^{*} \equiv \llbracket \mathrm{A} \rrbracket_{+}$.

We can now prove the main consequence of uniqueness of the deterministic effect $e_{G}$ for $\mathrm{A}_{G}$, i.e. that $e_{G}$ is the unique effect that amounts to 1 on $\llbracket \mathrm{A}_{G} \rrbracket_{1}$.
Proposition 8. Let $a \in \llbracket \overline{\mathrm{A}}_{G} \rrbracket_{Q}$. Then $(a \mid \rho)=1$ for all $\rho \in \llbracket \mathrm{A}_{G} \rrbracket_{1}$ if and only if $a=e_{G}$.

Proof. Notice that, since $e_{G} \succeq a$ for every $a \in$ $\llbracket \overline{\mathrm{A}}_{G} \rrbracket_{Q}$, and $(b \mid \rho) \geq 0$ for every $b \in \llbracket \overline{\mathrm{A}}_{G} \rrbracket_{Q}$ and $\rho \in \llbracket \mathrm{A}_{G} \rrbracket_{1}$, we have

$$
0 \leq\left(e_{G}-a \mid \rho\right)=1-(a \mid \rho),
$$

which implies $(a \mid \rho) \leq 1$ for every $\rho \in \llbracket \mathrm{A}_{G} \rrbracket_{1}$. Now, if $(a \mid \rho)=1$ for every $\rho \in \llbracket \mathrm{A}_{G} \rrbracket_{1}$, we have

$$
\left(e_{G}-a \mid \rho\right)=0, \quad \forall \rho \in \llbracket \mathrm{A}_{G} \rrbracket_{1},
$$

which by theorem 6 implies $a=e_{G}$.

A class of states of particular interest is that of quasi-local states, which are intuitively understood as states whose preparation can be arbitrarily approximated by local procedures. These live in small subspaces of the space $\llbracket \mathrm{A}_{G} \rrbracket_{\mathbb{R}}$. Their construction is similar to that of quasi-local effects, but differs form it in a relevant respect, that is the necessity of defining arbitrary reference states. The construction is inspired by pioneering works on infinite tensor products by von Neumann and Murray [49], and can be found in appendix B.

\subsection{Quasi-local transformations}

Now we are going to define the quasi-local algebra $\llbracket \mathrm{A}_{G} \rightarrow \mathrm{A}_{G} \rrbracket_{Q \mathbb{R}}$ of transformations. In usual approaches to quantum infinite systems, one usually introduces the effect algebra, that in the OPT case would correspond to the space of quasi-local effects. However, one can easily understand that, unlike effects in a general OPT, the quantum effect algebra contains far more information than the mere structure of the space of effects. Indeed, in the quantum effect algebra one can find every operator on the Hilbert space, and in turn, the operational role of a linear operator is to provide a Kraus representation of a transformation. In this perspective, one can also understand why multiplication of effects has a meaning at all: there is no point in multiplying effects, but multiplication of Kraus operators is the mathematical representation of sequential composition. Thus, the algebraic structure of effects conveniently summarises information about every kind of event in the theory: effects, with their coarse-graining represented by the sum, and transformations, with 
sequential composition represented by multiplication.

Cellular automata are defined in quantum theory by specifying their action on the effect algebra. Such an action thus provides information about how both effects and transformations are transformed by the cellular automaton. For a general OPT, however, such a compact algebraic structure embodying all the relevant information is absent, and the definition of a $\mathrm{CA}$ on the space of effects is not sufficient: we need to specify how the CA transforms transformations.

For these reasons we construct now the Banach algebra of quasi-local transformations, in a way that is very closely reminiscent of the construction of quasi-local effects. Here, in order to define a local transformation, we recur to the notion of a transformation that acts non-trivially on finitely many systems, while it acts as the identity on the remaining ones. In other words, the role that is played by the deterministic effect in the case of local effects is played here by the identity transformation. Also in this case, the topological closure will be taken in the sup-norm, and this choice is of great relevance to ensure a Banach algebra structure for the closure.

Let us then introduce the Banach algebra of quasi-local transformations.

Definition 20. Let $R \subseteq G$ be an arbitrary finite region of $G$. We define local transformation $\mathscr{A}_{R}$ of $\mathrm{A}_{G}$ any pair $(\mathscr{A}, R)$, where $\mathscr{A} \in \llbracket \mathrm{A}_{R} \rightarrow \mathrm{A}_{R} \rrbracket \mathbb{R}$. The action $\mathscr{A}_{R}^{\dagger}$ of $\mathscr{A}_{R}$ on Pre $\llbracket \overline{\mathrm{A}}_{G} \rrbracket L \mathbb{R}$ is defined as follows

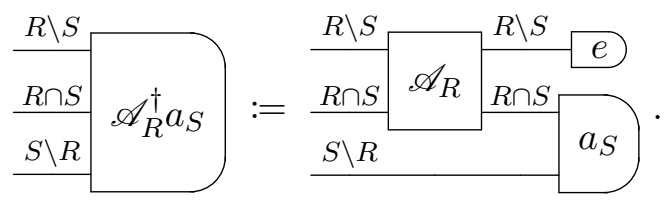

We also define Pre $\llbracket \mathrm{A}_{G} \rightarrow \mathrm{A}_{G} \rrbracket_{L \mathbb{R}}$ as the set of local transformations $\mathscr{A}_{R}$.

Lemma 21. Let $a_{S} \sim b_{T}$. Then $\mathscr{A}_{R}^{\dagger} a_{S} \sim \mathscr{A}_{R}^{\dagger} b_{T}$.

Proof. By hypothesis, there exists $c \in \llbracket \overline{\mathrm{A}}_{S \cap T} \rrbracket \mathbb{R}$ such that

$$
a=c \otimes e_{S \backslash T}, \quad b=c \otimes e_{T \backslash S} .
$$

Then we have

$$
\begin{aligned}
& \left(\mathscr{A}_{R}^{\dagger} a_{S} \mid=\left(c \otimes e_{S \backslash T} \otimes e_{R \backslash S} \mid\left(\mathscr{A} \otimes \mathscr{I}_{S \backslash R}\right),\right.\right. \\
& \left(\mathscr{A}_{R}^{\dagger} b_{T} \mid=\left(c \otimes e_{T \backslash S} \otimes e_{R \backslash T} \mid\left(\mathscr{A} \otimes \mathscr{I}_{T \backslash R}\right) .\right.\right.
\end{aligned}
$$

By Eq. (10) the thesis follows.
We can now define an equivalence relation between local transformations as follows

$$
\mathscr{A}_{S} \sim \mathscr{B}_{T} \Leftrightarrow\left\{\begin{array}{l}
\mathscr{A}=\mathscr{C} \otimes \mathscr{I}_{S \backslash T}, \\
\mathscr{B}=\mathscr{C} \otimes \mathscr{I}_{T \backslash S},
\end{array}\right.
$$

for some $\mathscr{C} \in \llbracket \mathrm{A}_{S \cap T} \rrbracket \mathbb{R}$. The set of local transformations is then defined as

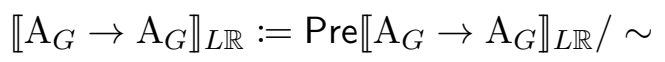

Lemma 22. Let $\mathscr{A}_{R} \in \operatorname{Pre} \llbracket \mathrm{A}_{G} \rightarrow \mathrm{A}_{G} \rrbracket L \mathbb{R}$. Then for every finite region $H \in \mathrm{R}^{(G)}$ such that $H \cap$ $R=\emptyset$, one has $\left(\mathscr{A} \otimes \mathscr{I}_{\mathrm{A}_{H}}\right)_{R \cup H} \in$ Pre $\llbracket \mathrm{A}_{G} \rightarrow$ $\mathrm{A}_{G} \rrbracket_{L \mathbb{R}}$, and $\left(\mathscr{A} \otimes \mathscr{I}_{\mathrm{A}_{H}}\right)_{R \cup H} \sim \mathscr{A}_{R}$.

Proof. It is straightforward to verify that $(\mathscr{A} \otimes$ $\left.\mathscr{I}_{\mathrm{A}_{H}}\right)_{R \cup H} \sim \mathscr{A}_{R}$ by direct inspection of the defining equation (41).

We can now provide a way to identify a canonical representative of the equivalence class of $\mathscr{A}_{R}$, which is defined in analogy to the case of effects as follows.

Definition 21. The minimal representative of the equivalence class $\mathscr{A}_{R}$, denoted as $\tilde{\mathscr{A}}_{R_{\mathscr{A}}}$, is defined through

$$
R_{\mathscr{A}}:=\bigcap_{S \in \mathrm{R}_{(\mathscr{A}, R)}} S, \quad \tilde{\mathscr{A}}_{R_{\mathscr{A}}} \sim \mathscr{A}_{R}
$$

where $\mathrm{R}_{(\mathscr{A}, R)}$ is the set of all those finite regions $S \subseteq G$ for which there exists $\mathscr{B} \in \llbracket \mathrm{A}_{S} \rightarrow \mathrm{A}_{S} \rrbracket_{\mathbb{R}}$ such that $\mathscr{B}_{S} \sim \mathscr{A}_{R}$.

Lemma 23. The minimal representative exists and is unique.

The proof follows step by step that of lemma 73.

The first result that we need to prove is the following.

Lemma 24. Let $\mathscr{A}_{R} \sim \mathscr{B}_{S}$. Then for every effect $a_{T} \in$ Pre $\llbracket \overline{\mathrm{A}}_{G} \rrbracket L \mathbb{R}$, one has

$$
\mathscr{A}_{R}^{\dagger} a_{T} \sim \mathscr{B}_{S}^{\dagger} a_{T}
$$

Proof. If $(\tilde{\mathscr{C}})_{R_{\mathscr{C}}}$ is the minimal representative of $\mathscr{A}_{R} \sim \mathscr{B}_{S}$, by Eq. (41), one has

$$
\begin{aligned}
& \mathscr{A}=\tilde{\mathscr{C}} \otimes \mathscr{I}_{R \backslash R_{\mathscr{C}}}, \\
& \mathscr{B}=\tilde{\mathscr{C}} \otimes \mathscr{I}_{S \backslash R_{\mathscr{C}}} .
\end{aligned}
$$


Now, by the defining equation 40 , we have that

$$
\begin{aligned}
& \left(\mathscr{A}_{R}^{\dagger} a_{T} \mid=\left(\tilde{a} \otimes e_{(T \cup R) \backslash R_{a}} \mid\left(\tilde{\mathscr{C}} \otimes \mathscr{I}_{(T \cup R) \backslash R_{\mathscr{C}}}\right)\right.\right. \\
= & \left(\tilde{a} \otimes e_{[T \cup(R \cap S)] \backslash R_{a}} \mid\left(\tilde{\mathscr{C}} \otimes \mathscr{I}_{[T \cup(R \cap S)] \backslash R_{\mathscr{C}}}\right)\right. \\
& \otimes\left(e_{R \backslash S} \mid,\right. \\
& \left(\mathscr{B}_{S}^{\dagger} a_{T} \mid=\left(\tilde{a} \otimes e_{(T \cup S) \backslash R_{a}} \mid\left(\tilde{\mathscr{C}} \otimes \mathscr{I}_{(T \cup S) \backslash R_{\mathscr{C}}}\right)\right.\right. \\
= & \left(\tilde{a} \otimes e_{[T \cup(R \cap S)] \backslash R_{a}} \mid\left(\tilde{\mathscr{C}} \otimes \mathscr{I}_{[T \cup(R \cap S)] \backslash R_{\mathscr{C}}}\right)\right. \\
& \otimes\left(e_{S \backslash R} \mid,\right.
\end{aligned}
$$

and finally by Eq. (123) this implies the thesis.

By virtue of lemmas 21 and 24, we can define the action of $\mathscr{A}_{R} \in \llbracket \mathrm{A}_{G} \rightarrow \mathrm{A}_{G} \rrbracket_{L \mathbb{R}}$ on local effects $\llbracket \overline{\mathrm{A}}_{G} \rrbracket_{\mathbb{R}}$ as

$$
\mathscr{A}_{R}^{\dagger}\left[a_{T}\right]:=\left[\mathscr{A}_{R}^{\dagger} a_{T}\right],
$$

where we leave the square braces to denote equivalence classes for $\sim$ in $\operatorname{Pre} \llbracket \overline{\mathrm{A}}_{G} \rrbracket_{L \mathbb{R}}$, for the sake of clarity. We can now make local transformations into a vector space as we did for local effects, as follows.

Definition 22. Let $\mathscr{A}_{R}, \mathscr{B}_{S} \in \llbracket \mathrm{A}_{G} \rightarrow \mathrm{A}_{G} \rrbracket_{L \mathbb{R}}$, and $h \in \mathbb{R}$. Then we define

$$
\begin{aligned}
& h \mathscr{A}_{R}:= \begin{cases}(h \mathscr{A})_{R} & h \neq 0, \\
0_{\emptyset} & h=0,\end{cases} \\
& \mathscr{A}_{R}+\mathscr{B}_{S}:=\mathscr{C}_{R \cup S} \\
& \mathscr{C}:=\mathscr{A} \otimes \mathscr{I}_{S \backslash R}+\mathscr{B} \otimes \mathscr{I}_{R \backslash S} .
\end{aligned}
$$

Moreover, the following operation makes the set of local transformations into an algebra.

Definition 23. Let $\mathscr{A}_{R}, \mathscr{B}_{S} \in \llbracket \mathrm{A}_{G} \rightarrow \mathrm{A}_{G} \rrbracket_{L \mathbb{R}}$. Then we define

$$
\mathscr{A}_{R} \mathscr{B}_{S}:=\left(\left\{\mathscr{A} \otimes \mathscr{I}_{S \backslash R}\right\}\left\{\mathscr{B} \otimes \mathscr{I}_{R \backslash S}\right\}\right)_{R \cup S} .
$$

We omit the straightforward proof that the above definition is well defined, i.e. independent of the choice of representatives in the classes of $\mathscr{A}_{R}$ and $\mathscr{B}_{S}$.

Definition 24. The algebra of generalized local transformations is the unital algebra $\llbracket \mathrm{A}_{G} \rightarrow$ $\mathrm{A}_{G} \rrbracket_{L \mathbb{R}}$ of finite real combinations of local transformations, with unit $1_{\emptyset}$ and null element $0_{\emptyset}$.
In order to close the algebra of local operations we introduce the topology given by the sup-norm, given in the following, in analogy to the case of effects. We also discuss the interplay of the supnorm topology with that given by the operational norm, that we define right away.

Definition 25. The operational norm $\left\|\mathscr{A}_{R}\right\|_{\text {op }}$ of $\tilde{\mathscr{A}}_{R_{\mathscr{A}}}=\mathscr{A}_{R} \in \llbracket \mathrm{A} \rightarrow \mathrm{A} \rrbracket_{L \mathbb{R}}$ is defined by the following expression

$$
\left\|\mathscr{A}_{R}\right\|_{\mathrm{op}}:=\|\tilde{\mathscr{A}}\|_{\mathrm{op}} .
$$

Proposition 9. Let $\mathscr{A}_{R} \in \llbracket \mathrm{A} \rightarrow \mathrm{A} \rrbracket_{L \mathbb{R}}$. Then $\left\|\mathscr{A}_{R}\right\|_{\mathrm{op}}=\|\mathscr{A}\|_{\mathrm{op}}$.

Proof. Let $\mathscr{A}_{R} \sim \tilde{\mathscr{A}}_{R_{\mathscr{A}}}$, and let $R=R_{\mathscr{A}} \cup R^{\prime}$, with $R_{\mathscr{A}} \cap R^{\prime}=\emptyset$. Then by Eq. (41) we have

$$
\mathscr{A}=\tilde{\mathscr{A}} \otimes \mathscr{I}_{\mathrm{A}_{R^{\prime}}},
$$

Now, by definition of $\|\cdot\|_{\text {op }}$ (see [8]) it straightforwardly follows that $\left\|\mathscr{B} \otimes \mathscr{I}_{\mathrm{C}}\right\|_{\text {op }}=\|\mathscr{B}\|_{\text {op }}$. Thus, $\left\|\mathscr{A}_{R}\right\|_{\text {op }}=\|\tilde{\mathscr{A}}\|_{\text {op }}=\|\mathscr{A}\|_{\text {op }}$.

Unfortunately, the operational norm does not enjoy the basic property that would make the algebra of transformations into a Banach algebra, i.e. it is not true that $\|\mathscr{A} \mathscr{B}\|_{\text {op }} \leq\|\mathscr{A}\|_{\text {op }}\|\mathscr{B}\|_{\text {op }}$. For this reason, in analogy with the case of quasilocal effects, we introduce a second norm, the supnorm, whose interplay with the operational norm will make it possible to define the closed Banach algebra of quasi-local transformations.

This is obtained extending the definition of sup-norm to the algebra of local transformations of $\mathrm{A}_{G}$.

Definition 26. The sup-norm $\left\|\mathscr{A}_{R}\right\|_{\text {sup }}$ of $\mathscr{A}_{R} \in$ $\llbracket \mathrm{A}_{G} \rightarrow \mathrm{A}_{G} \rrbracket_{L \mathbb{R}}$ is defined as

$$
\left\|\mathscr{A}_{R}\right\|_{\text {sup }}:=\inf J(\tilde{\mathscr{A}})=\|\tilde{\mathscr{A}}\|_{\text {sup }} .
$$

Proposition 10. Let $\mathscr{A}_{R} \in \llbracket \mathrm{A}_{G} \rightarrow \mathrm{A}_{G} \rrbracket_{L \mathbb{R}}$. Then $\left\|\mathscr{A}_{R}\right\|_{\text {sup }}=\|\mathscr{A}\|_{\text {sup }}$.

Proof. Let $\mathscr{A}_{R} \sim \tilde{\mathscr{A}}_{R_{\mathscr{A}}}$, and let $R=R_{\mathscr{A}} \cup R^{\prime}$, with $R_{\mathscr{A}} \cap R^{\prime}=\emptyset$. Then by Eq. (41) we have

$$
\mathscr{A}=\tilde{\mathscr{A}} \otimes \mathscr{I}_{\mathrm{A}_{R^{\prime}}},
$$

and by corollary 2, $\|\mathscr{A}\|_{\text {sup }}=\|\tilde{\mathscr{A}}\|_{\text {sup }}=$ $\left\|\mathscr{A}_{R}\right\|_{\text {sup }}$.

Corollary 10. Let $\mathscr{A}, \mathscr{B} \in \llbracket \mathrm{A}_{G} \rightarrow \mathrm{A}_{G} \rrbracket_{L \mathbb{R}}$. Then $\|\mathscr{A} \mathscr{B}\|_{\text {sup }} \leq\|\mathscr{A}\|_{\text {sup }}\|\mathscr{B}\|_{\text {sup }}$. 
Proof. The result is a straightforward consequence of propositions 10 and 2.

Corollary 11. On $\llbracket \mathrm{A}_{G} \rightarrow \mathrm{A}_{G} \rrbracket_{L \mathbb{R}}$, one has $\left\|\mathscr{A}_{R}\right\|_{\text {op }} \leq\left\|\mathscr{A}_{R}\right\|_{\text {sup }}$.

Proof. The result is a straightforward consequence of propositions 9 and 10, and corollary 4.

The above results allow us to extend the local algebra of events into the quasi-local algebra, which is a Banach algebra. The construction is analogous to that of quasi-local states, and proceeds as follows. First we define the algebra $\llbracket \mathrm{A}_{G} \rightarrow \mathrm{A}_{G} \rrbracket_{C \mathbb{R}}$ of sup-Cauchy sequences of local transformations, i.e. its elements are sequences $\mathscr{A}: \mathbb{N} \rightarrow \llbracket \mathrm{A}_{G} \rightarrow \mathrm{A}_{G} \rrbracket_{L \mathbb{R}}:: n \mapsto \mathscr{A}_{n R_{n}}$ such that for every $\varepsilon>0$ there exists $n_{0} \in \mathbb{N}$ such that for all $m, n \geq n_{0}$, one has $\left\|\mathscr{A}_{n}-\mathscr{A}_{m}\right\|_{\text {sup }}<\varepsilon$. Now we define the equivalence relation between elements of $\llbracket \mathrm{A}_{G} \rightarrow \mathrm{A}_{G} \rrbracket_{C \mathbb{R}}: \mathscr{A} \cong \mathscr{B}$ if for every $\varepsilon>0$ there exists $n_{0} \in \mathbb{N}$ such that for every $n \geq n_{0}$ one has $\left\|\mathscr{A}_{n}-\mathscr{B}_{n}\right\|_{\text {sup }}<\varepsilon$. Finally, we define the quasi-local algebra as follows.

Definition 27. The space of quasi-local transformations of $\mathrm{A}$ is defined as

$$
\llbracket \mathrm{A}_{G} \rightarrow \mathrm{A}_{G} \rrbracket_{Q \mathbb{R}}:=\llbracket \mathrm{A}_{G} \rightarrow \mathrm{A}_{G} \rrbracket_{C \mathbb{R}} / \cong .
$$

Definition 28. An element $\mathscr{A}$ in $\llbracket \mathrm{A}_{G} \rightarrow \mathrm{A}_{G} \rrbracket_{Q \mathbb{R}}$ is an event if $\mathscr{A}=\left[\mathscr{A}_{n R_{n}}\right]$ for a sequence $\mathscr{A}_{n R_{n}}$ such that $\mathscr{A}_{n} \in \llbracket \mathrm{A}_{R_{n}} \rightarrow \mathrm{A}_{R_{n}} \rrbracket$ for all $n \in \mathbb{N}$. The set of events will be denoted by $\llbracket \mathrm{A}_{G} \rightarrow \mathrm{A}_{G} \rrbracket_{Q}$.

An element $\mathscr{A} \in \llbracket \mathrm{A}_{G} \rightarrow \mathrm{A}_{G} \rrbracket_{Q}$ is a channel if $\mathscr{A}=\left[\mathscr{A}_{n R_{n}}\right]$ for a sequence $\mathscr{A}_{n R_{n}}$ such that $\mathscr{A}_{n} \in \llbracket \mathrm{A}_{R_{n}} \rightarrow \mathrm{A}_{R_{n}} \rrbracket_{1}$ for all $n \in \mathbb{N}$. The set of channels will be denoted by $\llbracket \mathrm{A}_{G} \rightarrow \mathrm{A}_{G} \rrbracket_{Q 1}$.

The subset of $\llbracket \mathrm{A}_{G} \rightarrow \mathrm{A}_{G} \rrbracket Q \mathbb{R}$ containing elements $\mathscr{A}=\left[\mathscr{A}_{n R_{n}}\right]$ for a sequence $\mathscr{A}_{n R_{n}}$ such that $\mathscr{A}_{n} \in \llbracket \mathrm{A}_{R_{n}} \rightarrow \mathrm{A}_{R_{n}} \rrbracket_{+}$for all $n \in \mathbb{N}$ will be denoted by $\llbracket \mathrm{A}_{G} \rightarrow \mathrm{A}_{G} \rrbracket_{Q+}$. Equivalently, we will write $\mathscr{A} \succeq 0$ for $\mathscr{A} \in \llbracket \mathrm{A}_{G} \rightarrow \mathrm{A}_{G} \rrbracket_{Q+}$

In the following, in analogy with the finite case, we will write $\mathscr{A} \succeq \mathscr{B}$ if $\mathscr{A}-\mathscr{B} \succeq 0$. The algebra of quasi-local transformations $\llbracket \mathrm{A}_{G} \rightarrow \mathrm{A}_{G} \rrbracket_{Q \mathbb{R}}$ is defined as follows.

Definition 29. Let $\mathscr{A}=\left[\mathscr{A}_{n R_{n}}\right], \mathscr{B}=\left[\mathscr{B}_{n S_{n}}\right]$. We define $\mathscr{A} \mathscr{B}:=\left[\mathscr{A}_{n R_{n}} \mathscr{B}_{n S_{n}}\right]$.

The product $\mathscr{A} \mathscr{B}$ is well defined. Indeed:
1. $\mathscr{A}_{n R_{n}} \mathscr{B}_{n S_{n}}$ is a Cauchy sequence.

2. For every $\mathscr{A}_{n R_{n}^{\prime}}^{\prime}, \mathscr{B}_{n S_{n}^{\prime}}^{\prime}$ in the equivalence classes defining $\mathscr{A}$ and $\mathscr{B}$, respectively, one has $\mathscr{A}_{n R_{n}} \mathscr{B}_{n S_{n}} \cong \mathscr{A}_{n R_{n}^{\prime}}^{\prime} \mathscr{B}_{n S_{n}^{\prime}}^{\prime}$.

Item 1 can be easily proved since we have

$$
\begin{aligned}
& \left\|\mathscr{A}_{n} \mathscr{B}_{n}-\mathscr{A}_{m} \mathscr{B}_{m}\right\|_{\text {sup }} \\
= & \left\|\mathscr{A}_{n} \mathscr{B}_{n}-\mathscr{A}_{m} \mathscr{B}_{n}+\mathscr{A}_{m} \mathscr{B}_{n}-\mathscr{A}_{m} \mathscr{B}_{m}\right\|_{\text {sup }} \\
\leq & \left\|\mathscr{A}_{n}-\mathscr{A}_{m}\right\|_{\text {sup }}\left\|\mathscr{B}_{n}\right\|_{\text {sup }} \\
& +\left\|\mathscr{A}_{m}\right\|_{\text {sup }}\left\|\mathscr{B}_{n}-\mathscr{B}_{m}\right\|_{\text {sup }} .
\end{aligned}
$$

Similarly, for item 2 we have

$$
\begin{aligned}
& \left\|\mathscr{A}_{n} \mathscr{B}_{n}-\mathscr{A}_{n}^{\prime} \mathscr{B}_{n}^{\prime}\right\|_{\text {sup }} \\
= & \left\|\mathscr{A}_{n} \mathscr{B}_{n}-\mathscr{A}_{n}^{\prime} \mathscr{B}_{n}+\mathscr{A}_{n}^{\prime} \mathscr{B}_{n}-\mathscr{A}_{n}^{\prime} \mathscr{B}_{n}^{\prime}\right\|_{\text {sup }} \\
\leq & \left\|\mathscr{A}_{n}-\mathscr{A}_{n}^{\prime}\right\|_{\text {sup }}\left\|\mathscr{B}_{n}\right\|_{\text {sup }} \\
& +\left\|\mathscr{A}_{n}^{\prime}\right\|_{\text {sup }}\left\|\mathscr{B}_{n}-\mathscr{B}_{n}^{\prime}\right\|_{\text {sup }} .
\end{aligned}
$$

The quasi-local algebra is actually a real Banach algebra if equipped with the norm $\|\cdot\|_{\text {sup }}$, as we now prove.

Proposition 11. The algebra $\llbracket \mathrm{A}_{G} \rightarrow \mathrm{A}_{G} \rrbracket_{Q \mathbb{R}}$ equipped with the norm $\|\cdot\|_{\text {sup }}$ is a Banach algebra.

Proof. We only need to prove that $\|\mathscr{A} \mathscr{B}\|_{\text {sup }} \leq$ $\|\mathscr{A}\|_{\text {sup }}\|\mathscr{B}\|_{\text {sup }}$. Let $\mathscr{A}=\left[\mathscr{A}_{n R_{n}}\right]$ and $\mathscr{B}=$ $\left[\mathscr{B}_{n S_{n}}\right]$, respectively. Now, for every $\mathscr{A}_{n R_{n}}$ and $\mathscr{B}_{n S_{n}}$ one has

$$
\begin{aligned}
& \left\|\left(\mathscr{A}_{n} \otimes \mathscr{I}_{S_{n} \backslash R_{n}}\right)\left(\mathscr{B}_{n} \otimes \mathscr{I}_{R_{n} \backslash S_{n}}\right)\right\|_{\text {sup }} \\
& \leq\left\|\mathscr{A}_{n} \otimes \mathscr{I}_{S_{n} \backslash R_{n}}\right\|_{\text {sup }}\left\|\mathscr{B}_{n} \otimes \mathscr{I}_{R_{n} \backslash S_{n}}\right\|_{\text {sup }},
\end{aligned}
$$

and by proposition $10\|\mathscr{A} \mathscr{B}\|_{\text {sup }} \leq$ $\|\mathscr{A}\|_{\text {sup }}\|\mathscr{B}\|_{\text {sup }}$.

The subsets $\llbracket \mathrm{A}_{G} \rightarrow \mathrm{A}_{G} \rrbracket_{Q}$ and $\llbracket \mathrm{A}_{G} \rightarrow \mathrm{A}_{G} \rrbracket_{Q 1}$ are convex, and $\llbracket \mathrm{A}_{G} \rightarrow \mathrm{A}_{G} \rrbracket_{Q+}$ is a convex cone. Moreover, they are closed in the sup-norm.

Proposition 12. The sets $\llbracket \mathrm{A}_{G} \rightarrow \mathrm{A}_{G} \rrbracket_{Q}$, $\llbracket \mathrm{A}_{G} \rightarrow \mathrm{A}_{G} \rrbracket_{Q 1}$, and $\llbracket \mathrm{A}_{G} \rightarrow \mathrm{A}_{G} \rrbracket_{Q+}$ are closed in the sup-norm.

Proof. The argument is the same in both cases. Let $\left\{\mathscr{A}_{m}\right\}_{m \in \mathbb{N}}$ be a Cauchy sequence with $\mathscr{A}_{m} \in \llbracket \mathrm{A}_{G} \rightarrow \mathrm{A}_{G} \rrbracket_{Q *}$ for all $m$, with $*=$ "nothing", $1,+$. By definition, $\mathscr{A}_{m}=\left[\mathscr{A}_{m n R_{m n}}\right]$, with $\mathscr{A}_{m n} \in \llbracket \mathrm{A}_{R_{m n}} \rightarrow \mathrm{A}_{R_{m n}} \rrbracket_{*}$. Then one easily proves that the sequence $\left\{\mathscr{A}_{m m R_{m m}}\right\}_{m \in \mathbb{N}}$ is Cauchy, and its limit is $\mathscr{A}:=\lim _{m \rightarrow \infty} \mathscr{A}_{m m R_{m m}}$, which by definition is in $\llbracket \mathrm{A}_{G} \rightarrow \mathrm{A}_{G} \rrbracket_{Q *}$. 
Proposition 13. Let $\mathscr{A} \in \llbracket \mathrm{A}_{G} \rightarrow \mathrm{A}_{G} \rrbracket_{Q \mathbb{R}}$. Then

$$
\|\mathscr{A}\|_{\text {op }} \leq\|\mathscr{A}\|_{\text {sup }}
$$

Proof. By definition $\|\mathscr{A}\|_{\text {op }}=\lim _{n \rightarrow \infty}\left\|\mathscr{A}_{n}\right\|_{\text {op }}$ and $\|\mathscr{A}\|_{\text {sup }}=\lim _{n \rightarrow \infty}\left\|\mathscr{A}_{n}\right\|_{\text {sup }}$, where $\left\{\mathscr{A}_{n R_{n}}\right\}_{n \in \mathbb{N}} \subseteq \llbracket \mathrm{A}_{G} \rightarrow \mathrm{A}_{G} \rrbracket_{L \mathbb{R}}$ is a Cauchy sequence converging to $\mathscr{A}$, thus by proposition 3 one has

$$
\left\|\mathscr{A}_{n}\right\|_{\text {op }} \leq\left\|\mathscr{A}_{n}\right\|_{\text {sup }}, \quad \forall n \in \mathbb{N},
$$

which implies the inequality in the limit.

Now, we can prove the following lemma.

Lemma 25. Let $a \in \llbracket \overline{\mathrm{A}}_{G} \rrbracket_{C \mathbb{R}}$ be a Cauchy sequence, and let $\mathscr{A} \in \llbracket \mathrm{A}_{G} \rightarrow \mathrm{A}_{G} \rrbracket_{L \mathbb{R}}$. We then have

$$
\mathscr{A}^{\dagger} a \in \llbracket \overline{\mathrm{A}}_{G} \rrbracket_{C \mathbb{R}} .
$$

Proof. We just need to evaluate $\left\|\mathscr{A}^{\dagger} a_{m}-\mathscr{A}^{\dagger} a_{n}\right\|_{\text {sup }}$, and using proposition 2 one has

$$
\left\|\mathscr{A}^{\dagger}\left(a_{m}-a_{n}\right)\right\|_{\text {sup }} \leq\left\|a_{m}-a_{n}\right\|_{\text {sup }}\|\mathscr{A}\|_{\text {sup }} .
$$

Since the sequence $a$ is Cauchy, also $\mathscr{A}^{\dagger} a$ is Cauchy.

Lemma 26. Let $a, b \in \llbracket \overline{\mathrm{A}}_{G} \rrbracket_{C \mathbb{R}}$ be equivalent Cauchy sequences, and let $\mathscr{A} \in \llbracket \mathrm{A}_{G} \rightarrow \mathrm{A}_{G} \rrbracket L \mathbb{R}$. We then have

$$
\left[\mathscr{A}^{\dagger} a_{n R_{n}}\right]=\left[\mathscr{A}^{\dagger} b_{n S_{n}}\right] .
$$

Proof. We can bound $\left\|\mathscr{A}^{\dagger} a_{n}-\mathscr{A}^{\dagger} b_{n}\right\|_{\text {sup }}$ using proposition 2, obtaining

$$
\left\|\mathscr{A}^{\dagger}\left(a_{n}-b_{n}\right)\right\|_{\text {sup }} \leq\left\|a_{n}-b_{n}\right\|_{\text {sup }}\|\mathscr{A}\|_{\text {sup }} .
$$

Since $\left[a_{n R_{n}}\right]=\left[b_{n S_{n}}\right]$, the thesis follows.

As a consequence of the above results, all local transformations leave the space $\llbracket \overline{\mathrm{A}}_{G} \rrbracket_{Q \mathbb{R}}$ invariant. We now prove a further important result: the above statement can be extended to all the quasi-local algebra.

Theorem 7. The quasi-local algebra $\llbracket \mathrm{A}_{G} \rightarrow$ $\mathrm{A}_{G} \rrbracket_{Q \mathbb{R}}$ leaves the space $\llbracket \overline{\mathrm{A}}_{G} \rrbracket_{Q \mathbb{R}}$ invariant, and for every $\mathscr{A} \in \llbracket \mathrm{A}_{G} \rightarrow \mathrm{A}_{G} \rrbracket_{Q \mathbb{R}}$ and every $a \in$ $\llbracket \overline{\mathrm{A}}_{G} \rrbracket_{Q \mathbb{R}}$, it is

$$
\left\|\mathscr{A}^{\dagger} a\right\|_{\text {sup }} \leq\|a\|_{\text {sup }}\|\mathscr{A}\|_{\text {sup }}
$$

Proof. By proposition 2 and lemma 25, the thesis is true for $\mathscr{A} \in \llbracket \mathrm{A}_{G} \rightarrow \mathrm{A}_{G} \rrbracket_{L \mathbb{R}}$ and $a \in \llbracket \overline{\mathrm{A}}_{G} \rrbracket_{L \mathbb{R}}$. Let now $a=\left[a_{p R_{p}}\right]$ and $\mathscr{A} \in \llbracket \mathrm{A}_{G} \rightarrow \mathrm{A}_{G} \rrbracket_{L \mathbb{R}}$. Then

$$
\left\|\mathscr{A}^{\dagger} a_{p}\right\|_{\text {sup }} \leq\left\|a_{p}\right\|_{\text {sup }}\|\mathscr{A}\|_{\text {sup }},
$$

and taking the limit for $p \rightarrow \infty$ we obtain the thesis. Finally, let $\mathscr{A}=\left[\mathscr{A}_{n R_{n}}\right]$. In this case, by lemma $25 \mathscr{A}_{n}^{\dagger} a \in \llbracket \overline{\mathrm{A}}_{G} \rrbracket_{Q \mathbb{R}}$ for every $n \in \mathbb{N}$. Moreover, $\left\{\mathscr{A}_{n}^{\dagger} a\right\}_{n \in \mathbb{N}} \in \llbracket \overline{\mathrm{A}}_{G} \rrbracket_{C \mathbb{R}}$. Indeed, by the result we just proved

$$
\left\|\left(\mathscr{A}_{n}^{\dagger}-\mathscr{A}_{m}^{\dagger}\right) a\right\|_{\text {sup }} \leq\|a\|_{\text {sup }}\left\|\mathscr{A}_{n}-\mathscr{A}_{m}\right\|_{\text {sup }} .
$$

Finally, by lemma 25, we have

$$
\left\|\mathscr{A}_{n}^{\dagger} a\right\|_{\text {sup }} \leq\|a\|_{\text {sup }}\left\|\mathscr{A}_{n}\right\|_{\text {sup }},
$$

and taking the limit for $n \rightarrow \infty$ we obtain the thesis.

A remarkable result that will be important in the following is given by the following lemma.

Lemma 27. Let $\mathscr{A} \in \llbracket \mathrm{A}_{G} \rightarrow \mathrm{A}_{G} \rrbracket_{Q}$. Then for every $a \in \llbracket \overline{\mathrm{A}}_{G} \rrbracket_{Q}$ one has $\mathscr{A}^{\dagger} a \in \llbracket \overline{\mathrm{A}}_{G} \rrbracket_{Q}$.

Proof. By hypothesis, $\mathscr{A}=\left[\mathscr{A}_{n R_{n}}\right]$ and $a=$ $\left[a_{m S_{m}}\right]$, with $\mathscr{A}_{n} \in \llbracket \mathrm{A}_{R_{n}} \rightarrow \mathrm{A}_{R_{n}} \rrbracket$ and $a_{m} \in$ $\llbracket \overline{\mathrm{A}}_{S_{m}} \rrbracket$. Thus $\mathscr{A}_{n}^{\dagger} a_{n} \in \llbracket \overline{\mathrm{A}}_{R_{n} \cup S_{n}} \rrbracket$. Now, one can easily verify that $\lim _{n \rightarrow \infty} \mathscr{A}_{n}^{\dagger} a_{n}=\mathscr{A}^{\dagger} a$, which proves the statement.

In particular, one has the following condition.

Lemma 28. Let $\mathscr{A} \in \llbracket \mathrm{A}_{G} \rightarrow \mathrm{A}_{G} \rrbracket_{Q+}$. Then $\mathscr{A} \in \llbracket \mathrm{A}_{G} \rightarrow \mathrm{A}_{G} \rrbracket_{Q}$ iff $\mathscr{A}^{\dagger} e_{G} \in \llbracket \overline{\mathrm{A}}_{G} \rrbracket_{Q}$.

Proof. By definition, one has $\mathscr{A} \in \llbracket \mathrm{A}_{G} \rightarrow \mathrm{A}_{G} \rrbracket_{Q}$ if and only if $\mathscr{A}=\left[\mathscr{A}_{n R_{n}}\right]$ with $\mathscr{A}_{n} \in \llbracket \mathrm{A}_{R_{n}} \rightarrow$ $\mathrm{A}_{R_{n}} \rrbracket$, and by our assumptions the latter is equivalent to $a_{n}:=\mathscr{A}_{n}^{\dagger} e_{\mathrm{A}_{R_{n}}} \in \llbracket \overline{\mathrm{A}}_{R_{n}} \rrbracket$. Now, since $\left\|a_{n R_{n}}-a_{m R_{m}}\right\|_{\text {sup }} \leq\left\|\mathscr{A}_{n R_{n}}-\mathscr{A}_{m R_{m}}\right\|_{\text {sup }}$, one has that $\mathscr{A} \in \llbracket \mathrm{A}_{G} \rightarrow \mathrm{A}_{G} \rrbracket_{Q}$ if and only if $\mathscr{A}^{\dagger} e_{G}=\left[a_{n R_{n}}\right] \in \llbracket \overline{\mathrm{A}}_{G} \rrbracket_{Q}$.

Lemma 29. Let $\mathscr{A} \in \llbracket \mathrm{A} \rightarrow \mathrm{B} \rrbracket_{Q+}$, and $a:=$ $\mathscr{A}^{\dagger} e_{G}$. Then $\|\mathscr{A}\|_{\text {sup }}=\|a\|_{\text {sup }}$.

Proof. By definition, $\mathscr{A}=\left[\mathscr{A}_{n R_{n}}\right]$, with $\mathscr{A}_{n} \in$ $\llbracket \mathrm{A}_{R_{n}} \rightarrow \mathrm{A}_{R_{n}} \rrbracket_{+}$. Now, $\left\|\mathscr{A}_{n R_{n}}\right\|_{\text {sup }}=\left\|\mathscr{A}_{n}\right\|_{\text {sup }}$, and by lemma $7,\left\|\mathscr{A}_{n}\right\|_{\text {sup }}=\left\|\mathscr{A}_{n}^{\dagger} e_{R_{n}}\right\|_{\text {sup }}=$ $\left\|\mathscr{A}_{n R_{n}}^{\dagger} e_{G}\right\|_{\text {sup. Then, }}$

$$
\|\mathscr{A}\|_{\text {sup }}=\lim _{n \rightarrow \infty}\left\|\mathscr{A}_{n R_{n}}^{\dagger} e_{G}\right\|_{\text {sup }}=\left\|\mathscr{A}^{\dagger} e_{G}\right\|_{\text {sup }} \cdot \square
$$


On the same line, we can provide the following condition for $\mathscr{A} \in \llbracket \mathrm{A}_{G} \rightarrow \mathrm{A}_{G} \rrbracket_{Q+}$ to be a channel.

Lemma 30. Let $\mathscr{A} \in \llbracket \mathrm{A}_{G} \rightarrow \mathrm{A}_{G} \rrbracket_{Q+}$. Then $\mathscr{A} \in \llbracket \mathrm{A}_{G} \rightarrow \mathrm{A}_{G} \rrbracket_{Q 1}$ iff $\mathscr{A}^{\dagger} e_{G}=e_{G}$.

Proof. First, let $\mathscr{A}$ be a channel. By definition it must be $\mathscr{A}=\lim _{n \rightarrow \infty} \mathscr{A}_{n R_{n}}$, where $\mathscr{A}_{n R_{n}}$ are local channels. Thus, by theorem $3, \mathscr{A}_{n R_{n}}^{\dagger} e_{G}=$ $e_{G}$. It follows that $\mathscr{A}^{\dagger} e_{G}=\lim _{n \rightarrow \infty} \mathscr{A}_{n R_{n}}^{\dagger} e_{G}=$ $e_{G}$. Now, for the converse, by definition $\mathscr{A} \in$ $\llbracket \mathrm{A}_{G} \rightarrow \mathrm{A}_{G} \rrbracket_{Q+}$ iff $\mathscr{A}=\left[\mathscr{A}_{n R_{n}}\right]$ with $\mathscr{A}_{n R_{n}} \in$ $\llbracket \mathrm{A}_{R_{n}} \rightarrow \mathrm{A}_{R_{n}} \rrbracket_{+}$for all $n \in \mathbb{N}$. Let us take $n \geq n_{0}$ so that $\left\|\mathscr{A}_{n R_{n}}-\mathscr{A}\right\|_{\text {sup }} \leq \varepsilon$. Then we have

$$
\left|\left\|\mathscr{A}_{n R_{n}}\right\|_{\text {sup }}-\|\mathscr{A}\|_{\text {sup }}\right| \leq \varepsilon .
$$

Now, by lemma 29, since $\mathscr{A}^{\dagger} e_{G}=e_{G}$, we have $\|\mathscr{A}\|_{\text {sup }}=1$. Let us define $\mathscr{A}_{n R_{n}}^{\prime}:=$ $\mathscr{A}_{n R_{n}} /\left\|\mathscr{A}_{n R_{n}}\right\|_{\text {sup }}$. Clearly,

$\left\|\mathscr{A}_{n R_{n}}^{\prime}-\mathscr{A}_{n R_{n}}\right\|_{\text {sup }}=\left\|\mathscr{A}_{n R_{n}}\right\|_{\text {sup }}\left|1-1 /\left\|\mathscr{A}_{n R_{n}}\right\|_{\text {sup }}\right| \leq \varepsilon$

This implies that $\mathscr{A}_{n R_{n}}^{\prime}$ is a sequence converging to $\mathscr{A}$, with $\left\|\mathscr{A}_{n R_{n}}^{\prime}\right\|_{\text {sup }}=1$. As a consequence, there exist channels $\mathscr{C}_{n R_{n}}$ such that $\left(\mathscr{C}_{n}-\mathscr{A}_{n}^{\prime}\right)_{R_{n}} \succeq 0$. Again by lemma 7 ,

$$
\begin{aligned}
\left\|\left(\mathscr{C}_{n}-\mathscr{A}_{n}^{\prime}\right)_{R_{n}}\right\|_{\text {sup }} & =\left\|\left(\mathscr{C}_{n}-\mathscr{A}_{n}^{\prime}\right)_{R_{n}}^{\dagger} e_{R_{n}}\right\|_{\text {sup }} \\
& =\left\|e_{G}-\mathscr{A}_{n R_{n}}^{\prime \dagger} e_{G}\right\|_{\text {sup }} \\
& =\left\|\left(\mathscr{A}-\mathscr{A}_{n R_{n}}^{\prime}\right)^{\dagger} e_{G}\right\|_{\text {sup }} \\
& \leq\left\|\left(\mathscr{A}-\mathscr{A}_{n R_{n}}^{\prime}\right)\right\|_{\text {sup }} \leq \varepsilon .
\end{aligned}
$$

This implies that $\mathscr{A}=\left[\mathscr{A}_{n R_{n}}^{\prime}\right]=\left[\mathscr{C}_{n R_{n}}\right]$, and then $\mathscr{A} \in \llbracket \mathrm{A}_{G} \rightarrow \mathrm{A}_{G} \rrbracket_{1}$.

The following weak version of the converse of lemma 28 can be easily proved.

Lemma 31. Let $a \in \llbracket \overline{\mathrm{A}}_{G} \rrbracket_{L \mathbb{R}}$. Then there exists $\mathscr{A} \in \llbracket \mathrm{A}_{G} \rrbracket_{L \mathbb{R}}$ such that

$$
a=\mathscr{A}^{\dagger} e_{G} .
$$

Moreover, for $a \in \llbracket \overline{\mathrm{A}}_{G} \rrbracket_{L}, E q$. (50) is satisfied with $\mathscr{A} \in \llbracket \mathrm{A}_{G} \rightarrow \mathrm{A}_{G} \rrbracket L$.

Proof. Let $a=a_{R} \in \llbracket \overline{\mathrm{A}}_{G} \rrbracket_{L \mathbb{R}}$. Let us define $\left.\mathscr{A}_{R}:=\mid \sigma\right)\left(a \mid\right.$, where $\sigma \in \llbracket \mathrm{A}_{R} \rrbracket_{1}$ is an arbitrary deterministic state. Then clearly

$$
\mathscr{A}_{R}^{\dagger} e_{G}=a_{R} .
$$

It is also clear that, for $a=a_{R} \in \llbracket \overline{\mathrm{A}}_{G} \rrbracket_{L}$, the above defined transformation $\mathscr{A}_{R}$ is in $\llbracket \mathrm{A}_{G} \rrbracket_{L}$.
One can take a further step, and prove the following result that will be of crucial importance in the remainder.

Corollary 12. Let $a \in \llbracket \overline{\mathrm{A}}_{G} \rrbracket_{Q \mathbb{R}}$. Then there exists a sequence of quasi-local transformations $\mathscr{A}_{n R_{n}}$ such that $\lim _{n \rightarrow \infty} \mathscr{A}_{n R_{n}}^{\dagger} e_{G}=$ a. If $a \in$ $\llbracket \overline{\mathrm{A}}_{G} \rrbracket_{Q}$ the sequence can be found in $\llbracket \mathrm{A}_{G} \rightarrow$ $\mathrm{A}_{G} \rrbracket_{Q}$.

Notice that the sequence $\mathscr{A}_{n R_{n}}$ is not necessarily Cauchy, thus one cannot conclude that for every $a \in \llbracket \overline{\mathrm{A}}_{G} \rrbracket_{Q \mathbb{R}}$ there is $\mathscr{A} \in \llbracket \mathrm{A}_{G} \rightarrow \mathrm{A}_{G} \rrbracket_{Q \mathbb{R}}$ such that $a=\mathscr{A}^{\dagger} e_{G}$. However, the following lemma completes the picture.

Proposition 14. Let $a \in \llbracket \overline{\mathrm{A}}_{G} \rrbracket_{Q \mathbb{R}}$. Then there exists $\mathscr{A} \in \llbracket \mathrm{A}_{G} \rightarrow \mathrm{A}_{G} \rrbracket_{Q \mathbb{R}}$ such that

$$
a=\mathscr{A}^{\dagger} e_{G} .
$$

Moreover, for $a \in \llbracket \overline{\mathrm{A}}_{G} \rrbracket_{Q *}$ with $* \in$ $\{$ "nothing", $1,+\}$ one can find the above $\mathscr{A}$ in the set $\llbracket \mathrm{A}_{G} \rightarrow \mathrm{A}_{G} \rrbracket_{Q *}$.

Proof. Let us define the sets

$\mathrm{T}_{a}^{\varepsilon}:=\left\{\mathscr{A} \in \llbracket \mathrm{A}_{G} \rightarrow \mathrm{A}_{G} \rrbracket_{Q \mathbb{R}} \mid\left\|\mathscr{A}^{\dagger} e_{G}-a\right\|_{\text {sup }} \leq \varepsilon\right\}$.

The sets $\mathrm{T}_{a}^{\varepsilon}$ are not empty for every $a$ and every $\varepsilon>0$, as a consequence of corollary 12 . Moreover, they are closed. Indeed, let $\left\{\mathscr{A}_{n}\right\}_{n \in \mathbb{N}}$ be a Cauchy sequence in $\mathrm{T}_{a}^{\varepsilon}$. This implies that by definition $a_{n}:=\mathscr{A}_{n}^{\dagger} e_{G} \in \bar{B}_{\varepsilon}(a)$. Since the ball $\bar{B}_{\varepsilon}(a)$ is closed, also $\lim _{n \rightarrow \infty} a_{n}=\left(\lim _{n \rightarrow \infty} \mathscr{A}_{n}\right)^{\dagger} e_{G} \in$ $\bar{B}_{\varepsilon}(a)$, namely $\lim _{n \rightarrow \infty} \mathscr{A}_{n} \in \mathrm{T}_{a}^{\varepsilon}$. Finally, one clearly has that for $\varepsilon<\delta$ it is $\mathrm{T}_{a}^{\varepsilon} \subseteq \mathrm{T}_{a}^{\delta}$, and the diameter $d_{\varepsilon}:=\sup \left\{\|\mathscr{A}-\mathscr{B}\|_{\text {sup }} \mid \mathscr{A}, \mathscr{B} \in \mathrm{T}_{a}^{\varepsilon}\right\}$ is a non-decreasing function of $\varepsilon$. Let then

$$
\mathrm{T}_{a}^{0}:=\bigcap_{\varepsilon>0} \mathrm{~T}_{a}^{\varepsilon} .
$$

Either $\lim _{\varepsilon \rightarrow 0^{+}} d_{\varepsilon}>0$, in which case $\mathrm{T}_{a}^{0}$ is non empty, and each of its elements satisfies $\mathscr{A}^{\dagger} e_{g}=$ $a$, or the limit is 0 , in which case, by Cantor's intersection theorem for complete metric spaces, $\mathrm{T}_{a}^{0}=\{\mathscr{A}\}$ is a singleton, with $\mathscr{A}^{\dagger} e_{G}=a$. The same argument can be applied in each of the cases where $a \in \llbracket \overline{\mathrm{A}}_{G} \rrbracket_{Q *}$, considering the closed sets $\mathrm{T}_{a}^{\varepsilon *}:=\mathrm{T}_{a}^{\varepsilon} \cap \llbracket \mathrm{A}_{G} \rightarrow \mathrm{A}_{G} \rrbracket_{Q *}$.

We now prove a result that is analogous to lemma 12. 
Lemma 32. Let $\mathscr{A} \in \llbracket \mathrm{A}_{G} \rightarrow \mathrm{A}_{G} \rrbracket_{Q+}$, and $\mathscr{C}-\mathscr{A} \in \llbracket \overline{\mathrm{A}}_{G} \rightarrow \mathrm{A}_{G} \rrbracket_{Q+}$ for some $\mathscr{C} \in \llbracket \mathrm{A}_{G} \rightarrow$ $\mathrm{A}_{G} \rrbracket_{Q 1}$. Then $\mathscr{A} \in \llbracket \mathrm{A}_{G} \rightarrow \mathrm{A}_{G} \rrbracket_{Q}$.

Proof. By definition, there are three sequences $\mathscr{A}_{n R_{n}}, \mathscr{A}_{n S_{n}}^{\prime}$ in $\llbracket \mathrm{A}_{G} \rightarrow \mathrm{A}_{G} \rrbracket_{L}$ and $\mathscr{C}_{n T_{n}}$ in $\llbracket \mathrm{A}_{G} \rightarrow$ $\mathrm{A}_{G} \rrbracket_{L 1}$ such that $\lim _{n \rightarrow \infty} \mathscr{A}_{n}=\mathscr{A}, \lim _{n \rightarrow \infty} \mathscr{A}_{n}^{\prime}=$ $\mathscr{C}-\mathscr{A}$, and $\lim _{n \rightarrow \infty} \mathscr{C}_{n}=\mathscr{C}$. This implies that

$$
\left\|\mathscr{C}_{n}-\left(\mathscr{A}_{n}+\mathscr{A}_{n}^{\prime}\right)\right\|_{\text {sup }} \leq \varepsilon
$$

and consequently there is a sequence of channels $\mathscr{D}_{n}$ in $\llbracket \mathrm{A}_{G} \rightarrow \mathrm{A}_{G} \rrbracket_{L 1}$ such that

$$
\varepsilon \mathscr{D}_{n}+\mathscr{C}_{n}-\left(\mathscr{A}_{n}+\mathscr{A}_{n}^{\prime}\right) \succeq 0 .
$$

By the above equation, it is easy to check that

$$
\mathscr{F}_{n}-\frac{1}{1+\varepsilon} \mathscr{A}_{n} \succeq 0, \quad \mathscr{F}_{n}-\frac{1}{1+\varepsilon} \mathscr{A}_{n}^{\prime} \succeq 0,
$$

where $\mathscr{F}_{n}$ is the channel $\varepsilon /(1+\varepsilon) \mathscr{D}_{n}+1 /(1+\varepsilon) \mathscr{C}_{n}$. Thus, by theorem 5 , both $\mathscr{A}_{n} /(1+\varepsilon)$ and $\mathscr{A}_{n}^{\prime} /(1+$ $\varepsilon$ ) belong to $\llbracket \mathrm{A}_{G} \rightarrow \mathrm{A}_{G} \rrbracket_{L}$. Let $\varepsilon_{m}$ be a real Cauchy sequence converging to 0 , and consider the corresponding sequences $\mathscr{B}_{m}:=\mathscr{A}_{n_{m}} /(1+$ $\left.\varepsilon_{m}\right), \mathscr{B}_{m}^{\prime}:=\mathscr{A}_{n_{m}}^{\prime} /\left(1+\varepsilon_{m}\right)$. Since

$$
\begin{aligned}
\left\|\mathscr{B}_{m}-\mathscr{A}_{n_{m}}\right\|_{\text {sup }} & =\left|\frac{\varepsilon_{m}}{1+\varepsilon_{m}}\right|\left\|\mathscr{A}_{n_{m}}\right\|_{\text {sup }}, \\
\left\|\mathscr{B}_{m}^{\prime}-\mathscr{A}_{n_{m}}^{\prime}\right\|_{\text {sup }} & =\left|\frac{\varepsilon_{m}}{1+\varepsilon_{m}}\right|\left\|\mathscr{A}_{n_{m}}^{\prime}\right\|_{\text {sup }},
\end{aligned}
$$

one has $\mathscr{A}=\left[\mathscr{A}_{n R_{n}}\right]=\left[\mathscr{B}_{m R_{m}}\right]$ and $\mathscr{C}-\mathscr{A}=$ $\left[\mathscr{A}_{n S_{n}}^{\prime}\right]=\left[\mathscr{B}_{m S_{m}}^{\prime}\right]$, with $\mathscr{B}_{n}$ and $\mathscr{B}_{n}^{\prime}$ beloning to $\llbracket \mathrm{A}_{G} \rightarrow \mathrm{A}_{G} \rrbracket_{L}$. Thus, $\mathscr{A}, \mathscr{C}-\mathscr{A} \in \llbracket \mathrm{A}_{G} \rightarrow \mathrm{A}_{G} \rrbracket_{Q}$.

We will now define the action of the quasi-local algebra of transformations on quasi-local states. The action is defined by duality as follows.

Definition 30 (Dual action of the quasi-local algebra). Let $\mathscr{A} \in \llbracket \mathrm{A}_{G} \rightarrow \mathrm{A}_{G} \rrbracket_{Q \mathbb{R}}$. We define the map $\hat{\mathscr{A}}: \llbracket \mathrm{A}_{G} \rrbracket_{\mathbb{R}} \rightarrow \llbracket \mathrm{A}_{G} \rrbracket_{\mathbb{R}}$ as follows

$$
(a \mid \hat{\mathscr{A}} \rho):=\left(\mathscr{A}^{\dagger} a \mid \rho\right), \quad \forall a \in \llbracket \overline{\mathrm{A}}_{G} \rrbracket_{Q \mathbb{R}} .
$$

The first thing we need to prove is that the map $\hat{\mathscr{A}}: \llbracket \mathrm{A}_{G} \rrbracket_{\mathbb{R}} \rightarrow \llbracket \mathrm{A}_{G} \rrbracket_{\mathbb{R}}$ is linear and bounded.

Proposition 15. For every $\mathscr{A} \in \llbracket \mathrm{A}_{G} \rightarrow \mathrm{A}_{G} \rrbracket_{Q \mathbb{R}}$ the map $\hat{\mathscr{A}}: \llbracket \mathrm{A}_{G} \rrbracket_{\mathbb{R}} \rightarrow \llbracket \mathrm{A}_{G} \rrbracket_{\mathbb{R}}$ is linear and bounded.
Proof. As to linearity, it is sufficient to consider that

$$
\begin{aligned}
\left(a \mid \hat{\mathscr{A}}\left[x \rho_{1}+y \rho_{2}\right]\right) & =\left(\mathscr{A}^{\dagger} a \mid x \rho_{1}+y \rho_{2}\right) \\
& =x\left(\mathscr{A}^{\dagger} a \mid \rho_{1}\right)+y\left(\mathscr{A}^{\dagger} a \mid \rho_{2}\right) \\
& =\left(a \mid x \hat{\mathscr{A}}_{1}\right)+\left(a \mid y \hat{\mathscr{A}} \rho_{2}\right),
\end{aligned}
$$

which by definition implies

$$
\hat{\mathscr{A}}\left(x \rho_{1}+y \rho_{2}\right)=x \hat{\mathscr{A}} \rho_{1}+y \hat{\mathscr{A}} \rho_{2} .
$$

In order to prove boundedness, it is sufficient to consider Eq. (49), to conclude that

$$
\begin{aligned}
|(a \mid \hat{\mathscr{A}} \rho)| & =\left|\left(\mathscr{A}^{\dagger} a \mid \rho\right)\right| \leq\left\|\mathscr{A}^{\dagger} a\right\|_{\text {sup }}\|\rho\|_{*} \\
& \leq\|a\|_{\text {sup }}\|\mathscr{A}\|_{\text {sup }}\|\rho\|_{*},
\end{aligned}
$$

which implies that

$$
\|\hat{\mathscr{A}} \rho\|_{*} \leq\|\mathscr{A}\|_{\text {sup }}\|\rho\|_{*} .
$$

We now prove that $\llbracket \mathrm{A}_{G} \rrbracket Q \mathbb{R}$ is invariant under the quasi-local algebra, and more precisely, every space $\llbracket \mathrm{A}_{G} \rrbracket_{\rho_{0} Q \mathbb{R}}^{(B)}$ is invariant.

Theorem 8. Let $\mathscr{A} \in \llbracket \mathrm{A}_{G} \rightarrow \mathrm{A}_{G} \rrbracket_{Q \mathbb{R}}$. Then

$$
\hat{\mathscr{A}} \llbracket \mathrm{A}_{G} \rrbracket_{Q \mathbb{R}} \subseteq \llbracket \mathrm{A}_{G} \rrbracket_{Q \mathbb{R}},
$$

and for every $\left(B, \rho_{0}\right)$,

$$
\hat{\mathscr{A}} \llbracket \mathrm{A}_{G} \rrbracket_{\rho_{0} Q \mathbb{R}}^{(B)} \subseteq \llbracket \mathrm{A}_{G} \rrbracket_{\rho_{0} Q \mathbb{R}}^{(B)} .
$$

Proof. We start observing that, by Eq. (40), when we apply $\mathscr{A}_{R}^{\dagger} a_{S}$ to a local state $\tau_{T}$ in $\llbracket \mathrm{A}_{G} \rrbracket_{\rho_{0} Q \mathbb{R}}^{(B)}$, we obtain

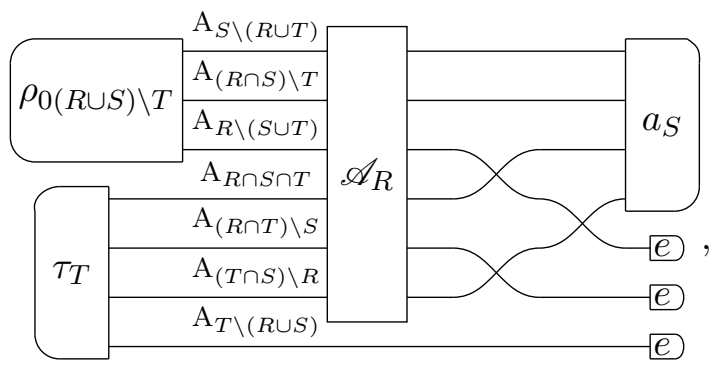

for every $S$ and every $a_{S} \in \llbracket \overline{\mathrm{A}}_{S} \rrbracket_{\mathbb{R}}$, which implies
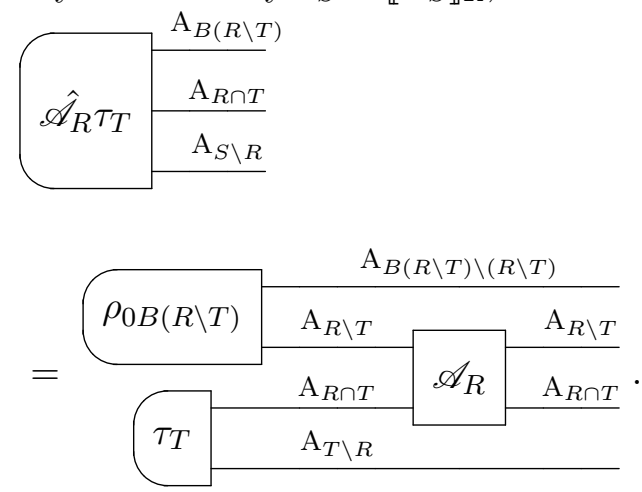
This means that local states in $\llbracket \mathrm{A}_{G} \rrbracket_{\rho_{0} Q \mathbb{R}}^{(B)}$ are mapped to local states in the same sector un$\operatorname{der} \hat{\mathscr{A}}_{R}$. Now, by proposition 15, Cauchy sequences are mapped to Cauchy sequences, so that $\hat{\mathscr{A}}_{R} \llbracket \mathrm{A}_{G} \rrbracket_{\rho_{0} Q \mathbb{R}}^{(B)} \subseteq \llbracket \mathrm{A}_{G} \rrbracket_{\rho_{0} Q \mathbb{R}}^{(B)}$. Moreover, again by proposition 15, also Cauchy sequences of local transformations satisfy the same condition, thus $\hat{\mathscr{A}} \llbracket \mathrm{A}_{G} \rrbracket_{\rho_{0} Q \mathbb{R}}^{(B)} \subseteq \llbracket \mathrm{A}_{G} \rrbracket_{\rho_{0} Q \mathbb{R}}^{(B)}$. Similarly, one can easily prove that $\hat{A} \llbracket \mathrm{A}_{G} \rrbracket_{Q \mathbb{R}} \subseteq \llbracket \mathrm{A}_{G} \rrbracket_{Q \mathbb{R}}$.

As a consequence of the above theorem, the action of $\llbracket \mathrm{A} \rightarrow \mathrm{A} \rrbracket_{Q}$ on the space $\llbracket \mathrm{A}_{G} \rrbracket_{Q \mathbb{R}}$ of quasilocal states is decomposed into many irreducible representations, one for every space $\llbracket \mathrm{A}_{G} \rrbracket_{\rho_{0} Q}^{(B)}$.

Remark 3. Notice that, given $\mathscr{A}, \mathscr{B} \in \llbracket \mathrm{A}_{G} \rightarrow$ $\mathrm{A}_{G} \rrbracket_{Q \mathbb{R}}$, one has

$$
(\mathscr{A} \mathscr{B})^{\dagger}=\mathscr{B}^{\dagger} \mathscr{A}^{\dagger}, \quad \widehat{(\mathscr{A} \mathscr{B})}=\hat{\mathscr{A}} \hat{\mathscr{B}} .
$$

Let $R \in \mathrm{R}^{(G)}$, and let $\mathscr{A} \in \llbracket \mathrm{A}_{G} \rightarrow \mathrm{A}_{G} \rrbracket_{Q}$. Suppose that $\mathscr{A}=\left[\mathscr{A}_{m R_{m}}\right]$ and for every $m \in \mathbb{N}$, $R \cap R_{m}=\emptyset$. This implies that, for $S_{m}=R_{m} \cup R$, $\mathscr{A}=\left[\mathscr{A}_{m S_{m}}^{\prime}\right]$, with $\mathscr{A}_{m S_{m}}^{\prime}=\left(\mathscr{A}_{m} \otimes \mathscr{I}_{R}\right)_{S_{m}}$, for $\mathscr{A}_{m}^{\prime} \in \llbracket \mathrm{A}_{S_{m} \backslash R} \rrbracket_{\mathbb{R}}$. We then write $\mathscr{A}=\mathscr{B} \otimes \mathscr{I}_{R}$. Notice that in this case, given $\rho \in \llbracket \mathrm{A}_{G} \rrbracket_{\mathbb{R}}$, one has

$$
\begin{aligned}
\left\|\rho_{\mid R}-(\hat{\mathscr{A}} \rho)_{\mid R}\right\|_{\text {op }} & =\left\|\left(\hat{\mathscr{A}}_{m} \rho\right)_{\mid R}-(\hat{\mathscr{A}} \rho)_{\mid R}\right\|_{\text {op }} \\
& \leq\left\|\left(\hat{\mathscr{A}}_{m}-\hat{\mathscr{A}}\right) \rho\right\|_{*} \leq \varepsilon\|\rho\|_{*},
\end{aligned}
$$

and then $(\hat{\mathscr{A}} \rho)_{\mid R}=\rho_{\mid R}$.

For $R \in \mathrm{R}^{(G)}$, there is a straightforward ordered Banach algebra isomorphism between $\llbracket \mathrm{A}_{R} \rightarrow \mathrm{A}_{R} \rrbracket_{Q \mathbb{R}}$ and $\llbracket \mathrm{A}_{R}^{(G)} \rightarrow \mathrm{A}_{R}^{(G)} \rrbracket_{Q \mathbb{R}}$, where the latter is defined as

$$
\llbracket \mathrm{A}_{R}^{(G)} \rightarrow \mathrm{A}_{R}^{(G)} \rrbracket_{Q \mathbb{R}}:=\left\{\mathscr{A}_{R} \mid \mathscr{A} \in \llbracket \mathrm{A}_{R} \rightarrow \mathrm{A}_{R} \rrbracket_{\mathbb{R}}\right\} .
$$

A similar isomorphism can be found also in the case of infinite regions $R \in \overline{\mathrm{R}}^{(G)}$, where by definition $\llbracket \mathrm{A}_{R}^{(G)} \rightarrow \mathrm{A}_{R}^{(G)} \rrbracket_{Q \mathbb{R}}$ is the closed subalgebra of Cauchy classes $\left[\mathscr{A}_{n R_{n}}\right]$ with $R_{n} \subseteq R$ for every $n \in \mathbb{N}$. The isomorphism in this case is given by

$$
\llbracket \mathrm{A}_{R} \rightarrow \mathrm{A}_{R} \rrbracket_{Q \mathbb{R}}^{\dagger}=\mathscr{J}_{R}^{\dagger} \llbracket \mathrm{A}_{R}^{(G)} \rightarrow \mathrm{A}_{R}^{(G)} \rrbracket_{Q \mathbb{R}}^{\dagger} \mathscr{J}_{R}^{-1 \dagger},
$$

where $\mathscr{J}_{R}^{\dagger}: \llbracket \overline{\mathrm{A}}_{R}^{(G)} \rrbracket_{Q \mathbb{R}} \rightarrow \llbracket \overline{\mathrm{A}}_{R} \rrbracket_{Q \mathbb{R}}$ is defined in Eq. (25). In the following, when we want to specify that a given quasi-local transformation $\mathscr{A}$ is in the subalgebra $\llbracket \mathrm{A}_{R} \rightarrow \mathrm{A}_{R} \rrbracket_{Q \mathbb{R}}$, we will write $\mathscr{A}_{R}$.
Remark 4. Notice that every quasi-local transformation in $\llbracket \mathrm{A}_{R} \rightarrow \mathrm{A}_{R} \rrbracket_{Q \mathbb{R}}$ does not only represent a linear map on $\llbracket \overline{\mathrm{A}}_{R} \rrbracket_{Q \mathbb{R}}$, but a family of maps that represent the same transformation acting on $\llbracket \overline{\mathrm{A}}_{G} \rrbracket$ for every $R \subseteq G$. This is due to the isomorphism of $\llbracket \mathrm{A}_{R} \rightarrow \mathrm{A}_{R} \rrbracket_{Q \mathbb{R}}$ with the subalgebra $\llbracket \mathrm{A}_{R}^{(G)} \rightarrow \mathrm{A}_{R}^{(G)} \rrbracket_{Q \mathbb{R}}$ given in Eq. (55).

The following result shows that $\llbracket \mathrm{A}_{R}^{(G)} \rightarrow$ $\mathrm{A}_{R}^{(G)} \rrbracket_{Q \mathbb{R}}$ preserves the subspace $\llbracket \overline{\mathrm{A}}_{R}^{(G)} \rrbracket_{Q \mathbb{R}}$.

Lemma 33. Let $\mathscr{A} \in \llbracket \mathrm{A}_{R}^{(G)} \rightarrow \mathrm{A}_{R}^{(G)} \rrbracket_{Q \mathbb{R}}$. Then

$$
\mathscr{A}^{\dagger} \llbracket \overline{\mathrm{A}}_{R}^{(G)} \rrbracket_{Q \mathbb{R}} \subseteq \llbracket \overline{\mathrm{A}}_{R}^{(G)} \rrbracket_{Q \mathbb{R}}
$$

Proof. Let $\mathscr{A}_{n R_{n}}$ be a sequence in the class of $\mathscr{A}$, with $R_{n} \subseteq R$ for every $n$. Let $a_{m S_{m}}$ be a sequence in the class of $a \in \llbracket \overline{\mathrm{A}}_{R}^{(G)} \rrbracket_{Q \mathbb{R}}$, with $S_{m} \subseteq$ $R$ for all $m$. Then $\mathscr{A}^{\dagger} a=\left[\left(\mathscr{A}_{n}^{\dagger} a_{n}\right)_{R_{n} \cup S_{n}}\right]$, and for every $n, R_{n} \cup S_{n} \subseteq R$. This implies that $\mathscr{A}^{\dagger} a \in \llbracket \overline{\mathrm{A}}_{R}^{(G)} \rrbracket_{Q \mathbb{R}}$.

The following intuitive result states that an effect that is localised in the region $R \subseteq G$ is obtained by a quasi-local transformation that is localised in the same region. This has a straightforward proof that will be omitted.

Lemma 34. Let $a \in \llbracket \overline{\mathrm{A}}_{R}^{(G)} \rrbracket_{Q *}$, with $* \in$ $\{$ "nothing", $1,+, \mathbb{R}\}$. Then

$$
a=\mathscr{A}^{\dagger} e_{G},
$$

with $\mathscr{A} \in \llbracket \mathrm{A}_{R}^{(G)} \rightarrow \mathrm{A}_{R}^{(G)} \rrbracket_{Q *}$.

We conclude the section with a result that confirms, under restrictive hypotheses, an obvious expectation.

Lemma 35. Let $\mathscr{A} \in \llbracket \mathrm{A}_{G} \rightarrow \mathrm{A}_{G} \rrbracket_{Q \mathbb{R}}$. Then $\|\mathscr{A}\|_{\text {sup }}=\inf J(\mathscr{A})$, where

$$
\begin{aligned}
& J(\mathscr{A}) \\
& :=\left\{\lambda \in \mathbb{R} \mid \exists \mathscr{C} \in \llbracket \mathrm{A}_{G} \rightarrow \mathrm{A}_{G} \rrbracket_{Q 1}, \lambda \mathscr{C} \pm \mathscr{A} \succeq 0\right\} .
\end{aligned}
$$

Proof. Let $\lambda \in J(\mathscr{A})$. Then there exists a quasilocal channel $\mathscr{C}$ such that $\lambda \mathscr{C} \pm \mathscr{A} \succeq 0$. By definition, this implies that $\lambda \mathscr{C} \pm \mathscr{A}=\left[\mathscr{B}_{n R_{n}^{ \pm}}^{ \pm}\right]$with $\mathscr{B}^{ \pm} \succeq 0$. Defining $\mathscr{A}_{n}:=1 / 2\left(\mathscr{B}_{n}^{+}-\mathscr{B}_{n}^{-}\right)$and $\mathscr{C}_{n}:=1 /(2 \lambda)\left(\mathscr{B}_{n}^{+}+\mathscr{B}_{n}^{-}\right)$, by construction one has

$$
\left[\mathscr{C}_{n S_{n}^{+}}\right]=\mathscr{C}, \quad\left[\mathscr{A}_{n S_{n}^{-}}\right]=\mathscr{A},
$$


where the domains $S_{n}^{ \pm}$are suitably defined. On the other hand, $\mathscr{C}=\left[\mathscr{D}_{n T_{n}}\right]$ for $\mathscr{D}_{n T_{n}} \in \llbracket \mathrm{A}_{T_{n}} \rightarrow$ $\mathrm{A}_{T_{n}} \rrbracket_{1}$. Thus, one has $\left\|\mathscr{C}_{n}-\mathscr{D}_{n}\right\|_{\text {sup }} \leq \varepsilon$, taking suitable care in defining the domain $U_{n}:=S_{n}^{+} \cup$ $T_{n}$ of $\mathscr{C}_{n}-\mathscr{D}_{n}$. In other terms, one can find a channel $\mathscr{F}_{n}$ such that

$$
\begin{aligned}
& \varepsilon \mathscr{F}_{n}+\mathscr{D}_{n}-\mathscr{C}_{n} \succeq 0, \\
& \varepsilon \mathscr{F}_{n}-\mathscr{D}_{n}+\mathscr{C}_{n} \succeq 0 .
\end{aligned}
$$

In turn, this implies that

$$
\begin{aligned}
& (1+\varepsilon) e_{U_{n}}-c_{n} \succeq 0, \\
& (\varepsilon-1) e_{U_{n}}+c_{n} \succeq 0,
\end{aligned}
$$

where $c_{n}:=\mathscr{C}_{n}^{\dagger} e_{U_{n}}$. Considering the first of the above relations, we can define

$$
\left.\mathscr{G}_{n}:=\frac{1}{1+\varepsilon} \mathscr{C}_{n}+\mid \rho_{n}\right)\left(d_{n} \mid\right.
$$

where $d_{n}:=e_{U_{n}}-1 /(1+\varepsilon) c_{n} \succeq 0$ and $\rho_{n}$ is an arbitrary state in $\llbracket \mathrm{A}_{U_{n}} \rrbracket_{1}$. By our assumptions, since $\mathscr{G}_{n} \succeq 0$ and $\mathscr{G}_{n}^{\dagger} e_{U_{n}}=e_{U_{n}}, \mathscr{G}_{n}$ is a channel. Moreover, by construction we have that

$$
\left.\lambda(1+\varepsilon) \mathscr{G}_{n} \pm \mathscr{A}_{n}=\mathscr{B}_{n}^{ \pm}+\lambda(1+\varepsilon) \mid \rho_{n}\right)\left(d_{n} \mid \succeq 0,\right.
$$

thus $\lambda(1+\varepsilon) \in J\left(\mathscr{A}_{n}\right)$, i.e. $\lambda(1+\varepsilon) \geq\left\|\mathscr{A}_{n}\right\|_{\text {sup }}$. In the limit, we then have $\lambda \geq\|\mathscr{A}\|_{\text {sup }}$. On the other hand, if $\lambda \notin J(\mathscr{A})$, for every $\mathscr{C} \in \llbracket \mathrm{A}_{G} \rightarrow$ $\mathrm{A}_{G} \rrbracket_{Q 1}$ one has

$$
\lambda \mathscr{C}+\mathscr{A} \nsucceq 0 \vee \lambda \mathscr{C}-\mathscr{A} \nsucceq 0 .
$$

Since the sequence $\lambda \mathscr{C} \pm \mathscr{A}_{n}$ converges to $\lambda \mathscr{C} \pm \mathscr{A}$ for every sequence $\mathscr{A}_{n}$ converging to $\mathscr{A}$, and the positive cone is closed, for every $\mathscr{C}$ and every $\mathscr{A}_{n}$ as above, there must be $n_{0}$ such that for $n \geq n_{0}$

$$
\lambda \mathscr{C}+\mathscr{A}_{n} \nsucc 0 \vee \lambda \mathscr{C}-\mathscr{A}_{n} \nsucc 0 .
$$

This holds, in particular, for every local $\mathscr{C}_{n}$ with the same domain as $\mathscr{A}_{n}$, which implies $\lambda \notin$ $J\left(\mathscr{A}_{n}\right)$. As a consequence, $\lambda<\left\|\mathscr{A}_{n}\right\|_{\text {sup }}$, and finally $\lambda \leq\|\mathscr{A}\|_{\text {sup. }}$. It is now easy to conclude that $\inf J(\mathscr{A})=\|\mathscr{A}\|_{\text {sup }}$.

We now prove that the infimum defining the sup norm is actually a minimum, analogously to the case of effects. The proof in this case is less straightforward, and we first need the following lemma.
Lemma 36. Given $\mathscr{A} \in \llbracket \mathrm{A}_{G} \rightarrow \mathrm{A}_{G} \rrbracket_{Q \mathbb{R}}$, let $\mathrm{S}_{\mathscr{A}}^{\lambda} \subseteq \llbracket \mathrm{A}_{G} \rightarrow \mathrm{A}_{G} \rrbracket_{Q 1}$ be the set of channels $\mathscr{C}$ such that $\lambda \mathscr{C} \pm \mathscr{A} \succeq 0$. Then $\mathrm{S}_{\mathscr{A}}^{\lambda}$ is closed in the sup-norm.

Proof. The empty set is closed, so we will consider the case where $\mathrm{S}_{\mathscr{A}}^{\lambda}$ is not empty. Let $\left\{\mathscr{C}_{n}\right\}_{n \in \mathbb{N}}$ be a Cauchy sequence in $\mathrm{S}_{\mathscr{A}}^{\lambda}$. Then $\mathscr{D}_{n}^{ \pm}:=\lambda \mathscr{C}_{n} \pm \mathscr{A} \succeq 0$ are both Cauchy sequences, that by definition converge to $\lambda \mathscr{C} \pm \mathscr{A} \succeq 0$. By proposition 12, $\mathscr{C} \in \llbracket \mathrm{A}_{G} \rightarrow \mathrm{A}_{G} \rrbracket_{Q 1}$.

Proposition 16. Let $\mathscr{A} \in \llbracket \mathrm{A}_{G} \rightarrow \mathrm{A}_{G} \rrbracket_{Q \mathbb{R}}$. Then there exists a quasi local channel $\mathscr{C}$ such that

$$
\|\mathscr{A}\|_{\sup } \mathscr{C} \pm \mathscr{A} \succeq 0 .
$$

Proof. By lemma 35, for every $\varepsilon>0$ there exists $\mathscr{C}_{\varepsilon}$ such that $\left(\|\mathscr{A}\|_{\text {sup }}+\varepsilon\right) \mathscr{C}_{\varepsilon} \pm \mathscr{A} \succeq 0$. Let us then consider the non empty sets $\mathrm{S}_{\varepsilon}:=\mathrm{S}_{\mathscr{A}}^{\|\|_{\text {sup }}+\varepsilon}$, that are closed by lemma 36 . It is straightforward to verify that for $\delta<\varepsilon$ it is $S_{\delta} \subseteq \mathrm{S}_{\varepsilon}$. Let $d_{\varepsilon}$ be the diameter of $S_{\varepsilon}$, namely $d_{\varepsilon}:=\sup \left\{\left\|\mathscr{C}_{1}-\mathscr{C}_{2}\right\|_{\text {sup }} \mid\right.$ $\left.\mathscr{C}_{1}, \mathscr{C}_{2} \in \mathrm{S}_{\varepsilon}\right\}$. Notice that $0 \leq d_{\varepsilon} \leq 2$. Being $d_{\varepsilon}$ a non-decreasing function of $\varepsilon, d_{0}=\lim _{\varepsilon \rightarrow 0^{+}}$ exists. The limit $d_{0}$ is the diameter of

$$
\mathrm{S}_{0}:=\bigcap_{\varepsilon>0} \mathrm{~S}_{\varepsilon} .
$$

If $d_{0}>0$, then $\mathrm{S}_{0}$ is clearly not empty, and each of its elements is a channel $\mathscr{C}$ such that $\|\mathscr{A}\|_{\text {sup }} \mathscr{C} \pm$ $\mathscr{A} \succeq 0$. If $d_{0}=0$, then by Cantor's intersection theorem for complete metric spaces the set $S_{0}$ is a singleton $\mathrm{S}_{0}=\left\{\mathscr{C}_{\infty}\right\}$, with $\|\mathscr{A}\|_{\sup } \mathscr{C}_{\infty} \pm \mathscr{A} \succeq$ 0 .

We finally prove a crucial result, that is the converse of lemma 32. This shows that every quasi-local event is an element of a quasi-local test.

Proposition 17. Let $\mathscr{A} \in \llbracket \mathrm{A}_{G} \rightarrow \mathrm{A}_{G} \rrbracket_{Q+}$. Then $\mathscr{A} \in \llbracket \mathrm{A}_{G} \rightarrow \mathrm{A}_{G} \rrbracket_{Q}$ if and only if there exists $\mathscr{C} \in \llbracket \mathrm{A}_{G} \rightarrow \mathrm{A}_{G} \rrbracket_{Q 1}$ such that $\mathscr{C}-\mathscr{A} \in$ $\llbracket \mathrm{A}_{G} \rightarrow \mathrm{A}_{G} \rrbracket_{Q+}$.

Proof. Sufficiency is proved in lemma 32. Let us then prove that if $\mathscr{A} \in \llbracket \mathrm{A}_{G} \rightarrow \mathrm{A}_{G} \rrbracket_{Q}$ then there exists $\mathscr{C} \in \llbracket \mathrm{A}_{G} \rightarrow \mathrm{A}_{G} \rrbracket_{Q 1}$ such that $\mathscr{C}-\mathscr{A} \succeq 0$. First of all, notice that $\mathscr{A}=\left[\mathscr{A}_{n R_{n}}\right]$ with $\mathscr{A}_{n} \in$ $\llbracket \mathrm{A}_{R_{n}} \rightarrow \mathrm{A}_{R_{n}} \rrbracket$, and then by

$$
\begin{aligned}
\|\mathscr{A}\|_{\text {sup }} & =\lim _{n \rightarrow \infty}\left\|\mathscr{A}_{n}\right\|_{\text {sup }} \\
& =\lim _{n \rightarrow \infty}\left\|\mathscr{A}_{n R_{n}}^{\dagger} e_{G}\right\|_{\text {sup }} \leq 1 .
\end{aligned}
$$


By proposition 16 , there exists $\mathscr{C} \in \llbracket \mathrm{A}_{G} \rightarrow$ $\mathrm{A}_{G} \rrbracket_{Q 1}$ such that

$$
\mathscr{C}-\mathscr{A} \succeq\|\mathscr{A}\|_{\text {sup }} \mathscr{C}-\mathscr{A} \succeq 0 .
$$

\section{Global update rules}

We have now set the theoretical background needed for the definition of a cellular automaton. In the present section we give the definition of an update rule (UR), that along with the system $\mathrm{A}_{G}$ - the array of cells, whose definition has been analyzed in detail in the previous sections - will provide the backbone of the notion of a cellular automaton. We then introduce the notion of a global update rule (GUR), as a family of update rules satisfying suitable admissibility conditions in order to represent a local action when extended to composite systems $\mathrm{A}_{G} \mathrm{C}$. A GUR thus describes the evolution occurring when we apply the global update rule on a joint state of $\mathrm{A}_{G}$ and $\mathrm{C}$, leaving $\mathrm{C}$ unaffected. We then analyse the main features of GURs, and in particular we focus on the causal influence relation given by a global update rule, and the block decomposition that allows one to calculate the action of a GUR on local effects using only local transformations.

Remark 5. In the remainder we will assume that for every $g \in G$ the system $\mathrm{A}_{g}$ is not trivial, namely $\mathrm{A}_{g} \nsucceq \mathrm{I}$.

First of all let $\mathscr{V}^{\dagger}$ be a bounded automorphism of the space $\llbracket \overline{\mathrm{A}}_{G} \rrbracket_{Q \mathbb{R}}$ of quasi-local effects. Then, with a notation that is reminiscent of the one adopted for quasi-local transformations, we will denote its action as

$$
\mathscr{V}^{\dagger}: \llbracket \overline{\mathrm{A}}_{G} \rrbracket_{Q \mathbb{R}} \rightarrow \llbracket \overline{\mathrm{A}}_{G} \rrbracket_{Q \mathbb{R}}:: a \mapsto \mathscr{V}^{\dagger} a .
$$

The action on effects allows us to define the dual map that acts on the space of extended states, as follows

$$
(a \mid \hat{\mathscr{V}} \rho):=\left(\mathscr{V}^{\dagger} a \mid \rho\right), \quad \forall a \in \llbracket \overline{\mathrm{A}}_{G} \rrbracket_{Q \mathbb{R}} .
$$

One can easily verify that $\hat{\mathscr{V}}$ is linear and bounded, thanks to boundedness of the map $\mathscr{V}^{\dagger}$. Unlike the usual notion of a map in quantum theory or theories with local discriminability, however, the action of $\hat{\mathscr{V}}$ or $\mathscr{V}^{\dagger}$ is not sufficient to identify uniquely the corresponding transformation. In particular, those actions are not sufficient to determine that of $\mathscr{V} \otimes \mathscr{I}_{\mathrm{C}}$, when system $\mathrm{A}_{G}$ is considered as a part of the composite system $\mathrm{A}_{G} \mathrm{C}$. For this reason we need to define an automorphic transformation of $\llbracket \overline{\mathrm{A}}_{G} \rrbracket_{Q \mathbb{R}}$ with care. One further delicate issue is the following. When one conjugates a quasi-local transformation $\mathscr{A}$ with an automorphism $\mathscr{V}^{\dagger}$, obtianing $\mathscr{A}^{\prime}=\mathscr{V}^{-1 \dagger} \mathscr{A}^{\dagger}$, the latter is a linear bounded map on $\llbracket \overline{\mathrm{A}}_{G} \rrbracket_{Q \mathbb{R}}$, but there is no guarantee that it is still an element of the quasi-local algebra $\llbracket \mathrm{A}_{G} \rightarrow \mathrm{A}_{G} \rrbracket_{Q \mathbb{R}}$, which is surely a desideratum for candidate cellular automata. In the remainder, when we write $\mathrm{A}_{G} \mathrm{C}$ we mean to deal with a system $\mathrm{A}_{G^{\prime}}$ with $G^{\prime}=G \cup h_{0}$ and $\mathrm{A}_{h_{0}} \cong$ C. In view of the above observations, we introduce the following notion

Definition 31 (Automorphic family). An automorphic family of maps on $\llbracket \overline{\mathrm{A}}_{G} \rrbracket_{Q \mathbb{R}}$ is a collection of automorphisms $\mathscr{V}_{\mathrm{C}}^{\dagger}$ of $\llbracket \overline{\mathrm{A}}_{G} \overline{\mathrm{C}} \rrbracket_{Q \mathbb{R}}$, one for every $\mathrm{C} \in \operatorname{Sys}(\Theta)$, with the properties $i$ ) for every $\mathscr{A} \in \llbracket \mathrm{A}_{C} \rightarrow \mathrm{A}_{C} \rrbracket_{\mathbb{R}}$ one has $\mathscr{V}_{\mathrm{C}}^{-1 \dagger} \mathscr{A}_{h_{0}}^{\dagger} \mathscr{V}_{\mathrm{C}}^{\dagger}=\mathscr{A}_{h_{0}}^{\dagger}$; ii) for fixed $\mathrm{C}_{0}$ and every choice of system $\mathrm{C}_{1}$, setting $\mathrm{C}=\mathrm{C}_{0} \mathrm{C}_{1}$ and $\mathrm{A}_{G^{\prime}}:=\mathrm{A}_{G} \mathrm{C}_{0}$, and for every $\mathscr{A} \in \llbracket \mathrm{A}_{G^{\prime}} \rightarrow \mathrm{A}_{G^{\prime}} \rrbracket_{Q \mathbb{R}}$, there exist $\mathscr{A}^{\prime}, \mathscr{A}^{\prime \prime} \in$ $\llbracket \mathrm{A}_{G^{\prime}} \rightarrow \mathrm{A}_{G^{\prime}} \rrbracket_{Q \mathbb{R}}$ such that

$$
\begin{aligned}
& \mathscr{V}_{\mathrm{C}}^{-1 \dagger} \mathscr{A}_{G^{\prime}}^{\dagger} \mathscr{V}_{\mathrm{C}}^{\dagger}=\mathscr{A}_{G^{\prime}}^{\prime \dagger}, \\
& \mathscr{A}_{G^{\prime}}^{\dagger}=\mathscr{V}_{\mathrm{C}}^{\dagger} \mathscr{A}_{G^{\prime}}^{\prime \prime \dagger} \mathscr{V}_{\mathrm{C}}^{-1 \dagger},
\end{aligned}
$$

where $\mathscr{A}^{\prime \dagger}=\left(\mathscr{V}_{\mathrm{C}_{0}}^{-1 \dagger} \mathscr{A}^{\dagger} \mathscr{V}_{\mathrm{C}_{0}}^{\dagger}\right)$, and $\mathscr{A}^{\prime \prime \dagger}=$ $\left(\mathscr{V}_{\mathrm{C}_{0}}^{-1 \dagger} \mathscr{A}^{\dagger} \mathscr{V}_{\mathrm{C}_{0}}^{\dagger}\right)$

We remark that, as a special case of item ii) in definition 31, taking $\mathrm{C}_{0} \equiv \mathrm{I}$ and $\mathrm{C}=\mathrm{C}_{1}$, for every choice of system $\mathrm{C}$, and every $\mathscr{A} \in \llbracket \mathrm{A}_{G} \rrbracket_{Q \mathbb{R}}$, one has

$$
\begin{aligned}
& \mathscr{V}_{\mathrm{C}}^{-1 \dagger} \mathscr{A}_{G}^{\dagger} \mathscr{V}_{\mathrm{C}}^{\dagger}=\left(\mathscr{V}_{\mathrm{I}}^{-1 \dagger} \mathscr{A}_{G}^{\dagger} \mathscr{V}_{\mathrm{I}}^{\dagger}\right)_{G}, \\
& \mathscr{V}_{\mathrm{C}}^{\dagger} \mathscr{A}_{G}^{\dagger} \mathscr{V}_{\mathrm{C}}^{-1 \dagger}=\left(\mathscr{V}_{\mathrm{I}}^{\dagger} \mathscr{A}_{G}^{\dagger} \mathscr{V}_{\mathrm{I}}^{-1 \dagger}\right)_{G} .
\end{aligned}
$$

When referring to an automorphic family we will use the shorthand $\mathscr{V}^{\dagger}$. One has to remind, however, that this symbol refers to a family of automorphisms. In particular, the definition is meant to allow for formulas such as $\mathscr{V} \mathscr{A} \mathscr{V}^{-1}$, $\mathscr{V} \llbracket \mathrm{A}_{G} \rightarrow \mathrm{A}_{G} \rrbracket_{\$_{*}} \mathscr{V}^{-1}$, for $\$=Q, L$ and $*=$ $\mathbb{R},+, 1$ or nothing. The meaning of the two expressions above is given by the following definitions:

$$
\begin{aligned}
& \mathscr{V} \mathscr{A} \mathscr{V}^{-1}:=\left\{\hat{\mathscr{V}}_{\mathrm{C}_{0} \mathrm{C}}\left(\hat{\mathscr{A}} \otimes \hat{\mathscr{I}}_{\mathrm{C}}\right) \hat{\mathscr{V}}_{\mathrm{C}_{0} \mathrm{C}}^{-1} \mid \mathrm{C} \in \operatorname{Sys}(\Theta)\right\}, \\
& \mathscr{V} \llbracket \mathrm{A}_{G^{\prime}} \rightarrow \mathrm{A}_{G^{\prime}} \rrbracket_{\$_{*}} \mathscr{V}^{-1}:= \\
& \quad\left\{\mathscr{V} \mathscr{A} \mathscr{V}^{-1} \mid \mathscr{A} \in \llbracket \mathrm{A}_{G^{\prime}} \rightarrow \mathrm{A}_{G^{\prime}} \rrbracket_{\$ *}\right\} .
\end{aligned}
$$


Notice that by definition of an automorphic family and by Eq. (62), one has

$$
\mathscr{V} \mathscr{A}^{-1}=\left\{\left(\hat{\mathscr{V}}_{\mathrm{C}_{0}} \hat{\mathscr{A}}_{\mathscr{V}_{\mathrm{C}}}^{-1}\right) \otimes \hat{\mathscr{I}}_{\mathrm{C}} \mid \mathrm{C} \in \operatorname{Sys}(\Theta)\right\},
$$

namely an automorphic family maps $\llbracket \mathrm{A}_{G^{\prime}} \rightarrow$ $\mathrm{A}_{G^{\prime}} \rrbracket_{Q \mathbb{R}}$ onto itself surjectively. In particular, this is true of $\llbracket \mathrm{A}_{G} \rightarrow \mathrm{A}_{G} \rrbracket_{Q \mathbb{R}}$. This implies that for $\mathscr{A} \in \llbracket \mathrm{A}_{G} \mathrm{C} \rightarrow \mathrm{A}_{G} \mathrm{C} \rrbracket_{Q \mathbb{R}}, \mathscr{V} \mathscr{A}_{\mathscr{V}}^{-1}$ represents a quasi-local transformation in $\llbracket \mathrm{A}_{G} \mathrm{C} \rightarrow \mathrm{A}_{G} \mathrm{C} \rrbracket_{Q \mathbb{R}}$.

An automorphic family is defined as to satisfy some necessary conditions that we require for the representatives of a transformation of the form $\mathscr{V} \otimes \mathscr{I}_{\mathrm{C}}$. However, the definition of an automorphic family is not sufficient to ensure consistency. This fact must be taken into account in the next subsection. Sufficient conditions will be only provided through the notion of admissibility.

\subsection{Update rule}

We can now use the notion of automorphic family to introduce update rules. An update rule is defined as a family of automorphisms, one for each extended system $\mathrm{A}_{G} \mathrm{C}$, with the constraint that the rule must act reversibly on the set of states and, by conjugation, on the cone of positive local transformations.

Definition 32 (Update rule). An update rule (UR) is a triple $\left(G, \mathrm{~A}, \mathscr{V}^{\dagger}\right)$, where $\mathscr{V}^{\dagger}$ is an automorphic family of isometric maps on $\llbracket \overline{\mathrm{A}}_{G} \rrbracket_{Q \mathbb{R}}$ such that

1. the maps $\hat{\mathscr{V}}_{\mathrm{C}}$ leave $\llbracket \mathrm{A}_{G} \mathrm{C} \rrbracket$ invariant, i.e.

$$
\hat{\mathscr{V}}_{\mathrm{C}} \llbracket \mathrm{A}_{G} \mathrm{C} \rrbracket=\llbracket \mathrm{A}_{G} \mathrm{C} \rrbracket ;
$$

2. the map $\mathscr{V} \cdot \mathscr{V}^{-1}$ leaves the cones $\llbracket \mathrm{A}_{G} \mathrm{C} \rightarrow$ $\mathrm{A}_{G} \mathrm{C} \rrbracket_{L+}$ invariant, i.e. for every $\mathscr{A} \in$ $\llbracket \mathrm{A}_{G} \mathrm{C} \rightarrow \mathrm{A}_{G} \mathrm{C} \rrbracket_{L+}$ there are $\mathscr{A}^{\prime}, \mathscr{A}^{\prime \prime} \in$ $\llbracket \mathrm{A}_{G} \mathrm{C} \rightarrow \mathrm{A}_{G} \mathrm{C} \rrbracket_{L+}$ such that

$$
\begin{aligned}
& \mathscr{V} \mathscr{A} \mathscr{V}^{-1}=\mathscr{A}^{\prime}, \\
& \mathscr{A}=\mathscr{V} \mathscr{A}^{\prime \prime} \mathscr{V}^{-1} .
\end{aligned}
$$

The first result is that the inverse of a UR is a UR.

Lemma 37. Let $\left(G, \mathrm{~A}, \mathscr{V}^{\dagger}\right)$ be a UR. Then $\left(G, \mathrm{~A}, \mathscr{V}^{-1 \dagger}\right)$ is a $U R$.
Proof. In the first place, the family $\mathscr{V}_{\mathrm{C}}^{-1}$ is an automorphic family: items i) and ii) in definition 31 are trivially proved. Now, if $\left\|\mathscr{V}_{\mathrm{C}}^{\dagger} a\right\|_{\text {sup }}=\|a\|_{\text {sup }}$ for every $a \in \llbracket \overline{\mathrm{A}}_{G} \overline{\mathrm{C}} \rrbracket_{Q \mathbb{R}}$, then $\left\|\mathscr{V}_{\mathrm{C}}^{-1 \dagger} a\right\|_{\text {sup }}=$ $\left\|\mathscr{V}_{\mathrm{C}}^{\dagger} \mathscr{V}_{\mathrm{C}}^{-1 \dagger} a\right\|_{\text {sup }}=\|a\|_{\text {sup }}$, which proves isometricity of $\mathscr{V}_{\mathrm{C}}^{-1 \dagger}$. From the definition in Eq. (59) one can easily verify that $\left(\hat{\mathscr{V}}_{\mathrm{C}}^{-1}\right)=\left(\hat{\mathscr{V}}_{\mathrm{C}}\right)^{-1}$. Now, it is a straightforward observation that by Eq. (65), $\hat{\mathscr{V}}_{\mathrm{C}}^{-1} \llbracket \mathrm{A}_{G} \mathrm{C} \rrbracket=\llbracket \mathrm{A}_{G} \mathrm{C} \rrbracket$. Finally, multiplying both sides of Eq. (66) by $\mathscr{V}^{-1}$ to the left and by $\mathscr{V}$ to the right, for every $\mathscr{A} \in \llbracket \mathrm{A}_{G} \mathrm{C} \rightarrow \mathrm{A}_{G} \mathrm{C} \rrbracket_{L+}$ one has

$$
\mathscr{A}=\mathscr{V}^{-1} \mathscr{A}^{\prime} \mathscr{V}
$$

which expresses the same condition as in Eq. (67) for $\mathscr{V}^{-1}$, and similarly, multiplying both sides of Eq. (67) by $\mathscr{V}^{-1}$ to the left and by $\mathscr{V}$ to the right, one obtains the same condition as in Eq. (66) for $\mathscr{V}^{-1}$.

In the following we will often use $\mathscr{V}^{\dagger}$ to denote a UR, when $G$ and $\mathrm{A}_{G}$ are clear from the context. The symbols $\hat{\mathscr{V}}$ and $\mathscr{V}^{\dagger}$ will be often used instead of $\hat{\mathscr{V}}_{\mathrm{C}}$ and $\mathscr{V}_{\mathrm{C}}^{\dagger}$, respectively. Accordingly, the symbols $a, b, \ldots$ and $\mathscr{A}, \mathscr{B}, \ldots$ will denote $a \otimes e_{\mathrm{C}}, b \otimes e_{\mathrm{C}}, \ldots$ and $\mathscr{A} \otimes \mathscr{I}_{\mathrm{C}}, \mathscr{B} \otimes \mathscr{I}_{\mathrm{C}}, \ldots$, respectively. Finally, we will sometimes write $G^{\prime}$ for $G \cup h_{0}$ with $\mathrm{A}_{h_{0}} \cong \mathrm{C}$, so that $\mathrm{A}_{G^{\prime}}=\mathrm{A}_{G} \mathrm{C}$.

The next result states that an update rule leaves the cone $\llbracket \mathrm{A}_{G}^{\left(G \cup h_{0}\right)} \rightarrow \mathrm{A}_{G}^{\left(G \cup h_{0}\right)} \rrbracket_{L+}$ invariant.

Lemma 38. Let $\left(G, \mathrm{~A}, \mathscr{V}^{\dagger}\right)$ be a UR, and define $\mathrm{A}_{G^{\prime}}:=\mathrm{A}_{G} \mathrm{C}$ for arbitrary $\mathrm{C} \in \operatorname{Sys}(\Theta)$. Then the map $\mathscr{V} \cdot \mathscr{V}^{-1}$ leaves the cone $\llbracket \mathrm{A}_{G}^{\left(G^{\prime}\right)} \rightarrow \mathrm{A}_{G}^{\left(G^{\prime}\right)} \rrbracket_{L+}$ invariant, namely for $\mathscr{A} \in \llbracket \mathrm{A}_{G}^{\left(G^{\prime}\right)} \rightarrow \mathrm{A}_{G}^{\left(G^{\prime}\right)} \rrbracket_{L+}$ Eqs. (66) and (67) hold with $\mathscr{A}^{\prime}, \mathscr{A}^{\prime \prime} \in \llbracket \mathrm{A}_{G}^{\left(G^{\prime}\right)} \rightarrow$ $\mathrm{A}_{G}^{\left(G^{\prime}\right)} \rrbracket_{L+}$.

Proof. Let $\mathscr{A} \in \llbracket \mathrm{A}_{G}^{G^{\prime}} \rightarrow \mathrm{A}_{G}^{G^{\prime}} \rrbracket_{L+} \subseteq \llbracket \mathrm{A}_{G^{\prime}} \rightarrow$ $\mathrm{A}_{G^{\prime}} \rrbracket_{L+}$. By definition of UR

$$
\mathscr{V} \mathscr{A}_{G} \mathscr{V}^{-1}=\mathscr{A}_{G}^{\prime} \in \llbracket \mathrm{A}_{G^{\prime}} \rightarrow \mathrm{A}_{G^{\prime}} \rrbracket_{L+} .
$$

Moreover, by definition of an automorphic family of maps and Eq. (64), we have

$$
\mathscr{A}^{\prime} \in \llbracket \mathrm{A}_{G}^{\left(G^{\prime}\right)} \rightarrow \mathrm{A}_{G}^{\left(G^{\prime}\right)} \rrbracket_{L \mathbb{R}} .
$$

The same holds exchanging $\mathscr{V}^{-1}$ and $\mathscr{V}$.

As a consequence of isometricity on $\llbracket \overline{\mathrm{A}}_{G} \overline{\mathrm{C}} \rrbracket_{Q \mathbb{R}}$, a UR $\mathscr{V}^{\dagger}$ has a dual $\hat{\mathscr{V}}$ that acts isometrically on $\llbracket \mathrm{A}_{G} \mathrm{C} \rrbracket_{\mathbb{R}}$. 
Lemma 39. Let $\mathscr{V}^{\dagger}$ be a UR. The maps $\hat{\mathscr{V}}_{\mathrm{C}}$ on $\llbracket \mathrm{A}_{G^{\prime}} \rrbracket_{\mathbb{R}}$ are isometric.

Proof. Let $\rho \in \llbracket \mathrm{A}_{G} \mathrm{C} \rrbracket \mathbb{R}$, and remind that

$$
\|\rho\|_{*}=\sup _{\|a\|_{\text {sup }}=1}|(a \mid \rho)| .
$$

Then we have

$$
\begin{aligned}
\left\|\hat{\mathscr{V}}_{\mathrm{C}} \rho\right\|_{*} & =\sup _{\|a\|_{\text {sup }}=1}\left|\left(a \mid \hat{\mathscr{V}}_{\mathrm{C}} \rho\right)\right|=\sup _{\|a\|_{\text {sup }}=1}\left|\left(\mathscr{V}_{\mathrm{C}}^{\dagger} a \mid \rho\right)\right| \\
& =\sup _{\left\|a^{\prime}\right\|_{\text {sup }}=1}\left|\left(a^{\prime} \mid \rho\right)\right|=\|\rho\|_{*},
\end{aligned}
$$

thanks to isometricity and invertibility of $\mathscr{V}_{\mathrm{C}}^{\dagger}$.

Corollary 13. Let $\mathscr{V}^{\dagger}$ be a UR. Then $\hat{\mathscr{V}}_{\mathrm{C}} \llbracket \mathrm{A}_{G} \mathrm{C} \rrbracket_{1}=\llbracket \mathrm{A}{ }_{G} \mathrm{C} \rrbracket_{1}$

Proof. By eqs. (65) and (29) and isometricity of $\hat{\mathscr{V}}_{\mathrm{C}}$, one has $\hat{\mathscr{V}}_{\mathrm{C}} \llbracket \mathrm{A}_{G} \mathrm{C} \rrbracket_{1} \subseteq \llbracket \mathrm{A}_{G} \mathrm{C} \rrbracket_{1}$. The same is true for $\hat{\mathscr{V}}_{\mathrm{C}}^{-1}$, thus $\hat{\mathscr{V}}_{\mathrm{C}} \llbracket \mathrm{A}_{G} \mathrm{C} \rrbracket_{1}=\llbracket \mathrm{A}_{G} \mathrm{C} \rrbracket_{1}$.

The next results will allow us to conclude that a UR preserves $\llbracket \overline{\mathrm{A}}_{G} \rrbracket_{Q}$. We start proving that an update rule $\mathscr{V}^{\dagger}$ preserves the unique deterministic effects $e_{G^{\prime}}$.

Lemma 40. If $\mathscr{V}^{\dagger}$ is a UR, then $\mathscr{V}^{\dagger} e_{G^{\prime}}=e_{G^{\prime}}$, for $\mathrm{A}_{G^{\prime}}:=\mathrm{A}_{G} \mathrm{C}$ and arbitrary $\mathrm{C} \in \operatorname{Sys}(\Theta)$.

Proof. By definition of a UR, for every $\rho \in \llbracket \mathrm{A}_{G^{\prime}} \rrbracket$ one has $\hat{\mathcal{V}} \rho \in \llbracket \mathrm{A}_{G^{\prime}} \rrbracket$, and lemma 19 , gives for every $\rho \in \llbracket \mathrm{A}_{G^{\prime}} \rrbracket$

$\left(\mathscr{V}^{\dagger} e_{G^{\prime}} \mid \rho\right)=\left(e_{G^{\prime}} \mid \hat{\mathscr{V}} \rho\right)=\|\hat{\mathscr{V}} \rho\|_{*}=\|\rho\|_{*}=\left(e_{G^{\prime}} \mid \rho\right)$.

Thanks to theorem 6 , we then have $\mathscr{V}^{\dagger} e_{G^{\prime}}=e_{G^{\prime}}$.

Corollary 14. Let $\mathscr{V}^{\dagger}$ be a UR. Then $\mathscr{V}^{\dagger} a_{h_{0}}=$ $a_{h_{0}}$ for all $a_{h_{0}} \in \llbracket \mathrm{A}_{h_{0}}^{\left(G^{\prime}\right)} \rightarrow \mathrm{A}_{h_{0}}^{\left(G^{\prime}\right)} \rrbracket_{Q \mathbb{R}}$.

Proof. By proposition 14, $a_{h_{0}}=\mathscr{A}_{h_{0}}^{\dagger} e_{G^{\prime}}$. Thus, by definition 31 and lemmas 40 and 56 , we have

$$
\begin{aligned}
\mathscr{V}^{\dagger} a_{h_{0}} & =\mathscr{V}^{\dagger} \mathscr{A}_{h_{0}}^{\dagger} \mathscr{V}^{-1 \dagger} e_{G^{\prime}} \\
& =\mathscr{A}_{h_{0}}^{\dagger} e_{G^{\prime}}=a_{h_{0}} .
\end{aligned}
$$

We now prove that the cones of effects $\llbracket \overline{\mathrm{A}}_{G^{\prime}} \rrbracket_{L+}$, $\llbracket \overline{\mathrm{A}}_{G} \rrbracket_{L+}, \llbracket \overline{\mathrm{A}}_{G^{\prime}} \rrbracket_{Q+}$ and $\llbracket \overline{\mathrm{A}}_{G} \rrbracket_{Q+}$ are invariant under the action of any UR.

Lemma 41. Let $\mathscr{V}^{\dagger}$ be a UR. Then $\mathscr{V}^{\dagger} \llbracket \overline{\mathrm{A}}_{G^{\prime}} \rrbracket_{L+}=\llbracket \overline{\mathrm{A}}_{G^{\prime}} \rrbracket_{L+}$, and $\mathscr{V}^{\dagger} \llbracket \overline{\mathrm{A}}_{G^{\prime}} \rrbracket_{Q+}=$ $\llbracket \overline{\mathrm{A}}_{G^{\prime}} \rrbracket_{Q+}$. In particular, for $\mathrm{C}=\mathrm{I}$ it is $\mathscr{V}^{\dagger} \llbracket \overline{\mathrm{A}}_{G} \rrbracket_{Q+}=\llbracket \overline{\mathrm{A}}_{G} \rrbracket_{Q+}$
Proof. Let $a \in \llbracket \overline{\mathrm{A}}_{G^{\prime}} \rrbracket_{L+}$. By proposition 14, there exists $\mathscr{A} \in \llbracket \mathrm{A}_{G^{\prime}} \rightarrow \mathrm{A}_{G^{\prime}} \rrbracket_{L+}$ such that $a=\mathscr{A}^{\dagger} e_{G^{\prime}}$. Then

$$
\begin{aligned}
\mathscr{V}^{\dagger} a & =\mathscr{V}^{\dagger} \mathscr{A}^{\dagger} e_{G^{\prime}} \\
& =\mathscr{V}^{\dagger} \mathscr{A}^{\dagger} \mathscr{V}^{-1 \dagger} \mathscr{V}^{\dagger} e_{G^{\prime}} \\
& =\mathscr{V}^{\dagger} \mathscr{A}^{\dagger} \mathscr{V}^{-1 \dagger} e_{G^{\prime}} \\
& =\mathscr{A}^{\prime \dagger} e_{G^{\prime}}=a^{\prime} .
\end{aligned}
$$

We observe that, by definition of UR and by lemma $38, \mathscr{V} \cdot \mathscr{V}^{-1}$ preserves the cones $\llbracket \mathrm{A}_{G^{\prime}} \rightarrow$ $\mathrm{A}_{G^{\prime}} \rrbracket_{L+}$. Thus, $a^{\prime} \in \llbracket \overline{\mathrm{A}}_{G^{\prime}} \rrbracket_{L+}$, and $\mathscr{V}^{\dagger} \llbracket \overline{\mathrm{A}}_{G^{\prime}} \rrbracket_{L+} \subseteq$ $\llbracket \overline{\mathrm{A}}_{G^{\prime}} \rrbracket_{L+}$. Since also $\mathscr{V}^{-1 \dagger}$ is a UR, we have $\mathscr{V}^{\dagger} \llbracket \overline{\mathrm{A}}_{G^{\prime}} \rrbracket_{L+}=\llbracket \overline{\mathrm{A}}_{G^{\prime}} \rrbracket_{L+}$. Now, isometricity grants that Cauchy sequences of local effects in $\llbracket \overline{\mathrm{A}}_{G^{\prime}} \rrbracket_{L+}$ are mapped to Cauchy sequences in $\llbracket \overline{\mathrm{A}}_{G^{\prime}} \rrbracket_{L+}$, and that $\lim _{n \rightarrow \infty} \mathscr{V}^{\dagger} a_{n}=\mathscr{V}^{\dagger} \lim _{n \rightarrow \infty} a_{n}$. Finally, by proposition $12, \mathscr{V}^{\dagger} \llbracket \overline{\mathrm{A}}_{G^{\prime}} \rrbracket_{Q+} \subseteq \llbracket \overline{\mathrm{A}}_{G^{\prime}} \rrbracket_{Q+}$. Since also $\mathscr{V}^{-1 \dagger}$ is a UR, we conclude that $\mathscr{V}^{\dagger} \llbracket \overline{\mathrm{A}}_{G^{\prime}} \rrbracket_{Q+}=\llbracket \overline{\mathrm{A}}_{G^{\prime}} \rrbracket_{Q+}$.

Proposition 18. Let $\mathscr{V}^{\dagger}$ be a UR. Then $\mathscr{V}^{\dagger} \llbracket \overline{\mathrm{A}}_{G^{\prime}} \rrbracket_{Q}=\llbracket \overline{\mathrm{A}}_{G^{\prime}} \rrbracket_{Q}$. In particular, for $\mathrm{C}=\mathrm{I}$, $\mathscr{V}^{\dagger} \llbracket \overline{\mathrm{A}}_{G} \rrbracket_{Q}=\llbracket \overline{\mathrm{A}}_{G} \rrbracket_{Q}$

Proof. Let $a \in \llbracket \overline{\mathrm{A}}_{G^{\prime}} \rrbracket_{Q}$. Then $e_{G^{\prime}}-a \in \llbracket \overline{\mathrm{A}}_{G^{\prime}} \rrbracket_{Q+}$, by lemma 12 , and thus, by virtue of lemmas 40 and $41, \mathscr{V}^{\dagger} a, e_{G^{\prime}}-\mathscr{V}^{\dagger} a \in \llbracket \overline{\mathrm{A}}_{G^{\prime}} \rrbracket_{Q+}$. Again by lemma 12, this implies that $\mathscr{V}^{\dagger} a \in \llbracket \overline{\mathrm{A}}_{G^{\prime}} \rrbracket_{Q}$, and thus $\mathscr{V}^{\dagger} \llbracket \overline{\mathrm{A}}_{G^{\prime}} \rrbracket_{Q} \subseteq \llbracket \overline{\mathrm{A}}_{G^{\prime}} \rrbracket_{Q}$. Finally, since also $\mathscr{V}^{-1 \dagger}$ is a UR, the thesis follows.

Let now $\mathscr{V}^{\dagger}$ be an update rule. By our definition, the map $\mathscr{V} \cdot \mathscr{V}^{-1}$ preserves the cones $\llbracket \mathrm{A}_{G^{\prime}} \rightarrow \mathrm{A}_{G^{\prime}} \rrbracket_{L+}$. We will now prove that $\mathscr{V} \cdot \mathscr{V}^{-1}$ also preserves the cones $\llbracket \mathrm{A}_{G^{\prime}} \rightarrow \mathrm{A}_{G^{\prime}} \rrbracket_{Q+}$, as well as the sets of transformations $\llbracket \mathrm{A}_{G^{\prime}} \rightarrow \mathrm{A}_{G^{\prime}} \rrbracket_{L}$ and $\llbracket \mathrm{A}_{G^{\prime}} \rightarrow \mathrm{A}_{G^{\prime}} \rrbracket_{Q}$.

We start with the following lemma

Lemma 42. Let $\mathscr{V}^{\dagger}$ be a UR. Then $\mathscr{V} \cdot \mathscr{V}^{-1}$ leaves the sets $\llbracket \mathrm{A}_{G^{\prime}} \rightarrow \mathrm{A}_{G^{\prime}} \rrbracket_{L 1}$ invariant, namely for $\mathscr{A} \in \llbracket \mathrm{A}_{G^{\prime}} \rightarrow \mathrm{A}_{G^{\prime}} \rrbracket L 1$ Eqs. (66) and (67) hold, with $\mathscr{A}^{\prime}, \mathscr{A}^{\prime \prime} \in \llbracket \mathrm{A}_{G^{\prime}} \rightarrow \mathrm{A}_{G^{\prime}} \rrbracket L 1$. In particular, for $\mathrm{C}=\mathrm{I}$ the thesis holds with $G^{\prime}=G$.

Proof. Let $\mathscr{C} \in \llbracket \mathrm{A}_{G^{\prime}} \rightarrow \mathrm{A}_{G^{\prime}} \rrbracket_{L 1}$. By definition and by lemma $38, \mathscr{V} \mathscr{C}^{-1}=\mathscr{C}^{\prime}$, and $\mathscr{C}=\mathscr{V} \mathscr{C}^{\prime \prime} \mathscr{V}^{-1}$, for $\mathscr{C}^{\prime}, \mathscr{C}^{\prime \prime} \in \llbracket \mathrm{A}_{G^{\prime}} \rightarrow \mathrm{A}_{G^{\prime}} \rrbracket_{L+}$. By lemmas 37 and 40,

$$
\begin{aligned}
& \mathscr{C}^{\prime \dagger} e_{G^{\prime}}=\mathscr{V}^{\dagger} \mathscr{C}^{\dagger} \mathscr{V}^{-1 \dagger} e_{G^{\prime}}=e_{G^{\prime}},
\end{aligned}
$$

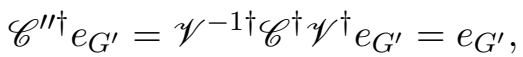


and due to lemma $30, \mathscr{C}^{\prime}, \mathscr{C}^{\prime \prime} \in \llbracket \mathrm{A}_{G^{\prime}} \rightarrow \mathrm{A}_{G^{\prime}} \rrbracket_{L 1}$.

Given a UR $\mathscr{V}^{\dagger}$, the above result allows us to prove that $\mathscr{V} \cdot \mathscr{V}^{-1}$ maps local transformations to local transformations, and is isometric on $\llbracket \mathrm{A}_{G^{\prime}} \rightarrow$ $\mathrm{A}_{G^{\prime}} \rrbracket_{L \mathbb{R}}$.

Lemma 43. Let $\mathscr{V}^{\dagger}$ be a $U R$, and $\mathscr{A} \in \llbracket \mathrm{A}_{G^{\prime}} \rightarrow$ $\mathrm{A}_{G^{\prime}} \rrbracket_{L \mathbb{R}}$. Then $\mathscr{V} \llbracket \mathrm{A}_{G^{\prime}} \rightarrow \mathrm{A}_{G^{\prime}} \rrbracket_{L \mathbb{R}} \mathscr{V}^{-1}=\llbracket \mathrm{A}_{G^{\prime}} \rightarrow$ $\mathrm{A}_{G^{\prime}} \rrbracket_{L \mathbb{R}}$, and

$$
\left\|\mathscr{V} \mathscr{A} \mathscr{V}^{-1}\right\|_{\text {sup }}=\|\mathscr{A}\|_{\text {sup }} .
$$

Proof. By proposition 16, there exists $\mathscr{C} \in$ $\llbracket \mathrm{A}_{G^{\prime}} \rightarrow \mathrm{A}_{G^{\prime}} \rrbracket_{L 1}$ such that

$$
\|\mathscr{A}\|_{\sup } \mathscr{C} \pm \mathscr{A} \succeq 0 .
$$

By definition of a UR, we then have

$$
\|\mathscr{A}\|_{\sup } \mathscr{V} \mathscr{C}^{-1} \pm \mathscr{V} \mathscr{A} \mathscr{V}^{-1} \succeq 0,
$$

and being $\mathscr{V} \mathscr{C}^{-1} \in \llbracket \mathrm{A}_{G^{\prime}} \rightarrow \mathrm{A}_{G^{\prime}} \rrbracket_{L 1} \subseteq$ $\llbracket \mathrm{A}_{G^{\prime}} \rightarrow \mathrm{A}_{G^{\prime}} \rrbracket_{L \mathbb{R}}$, it follows that $\mathscr{V} \mathscr{A} \mathscr{V}^{-1} \in$ $\llbracket \mathrm{A}_{G^{\prime}} \rightarrow \mathrm{A}_{G^{\prime}} \rrbracket_{L \mathbb{R}}$. Consequently, we have $\mathscr{V} \llbracket \mathrm{A}_{G^{\prime}} \rightarrow \mathrm{A}_{G^{\prime}} \rrbracket_{L \mathbb{R}} \mathscr{V}^{-1} \subseteq \llbracket \mathrm{A}_{G^{\prime}} \rightarrow \mathrm{A}_{G^{\prime}} \rrbracket_{L \mathbb{R}}$. Finally, since $\mathscr{V}^{-1 \dagger}$ is a UR, we have that $\mathscr{V} \llbracket \mathrm{A}_{G^{\prime}} \rightarrow \mathrm{A}_{G^{\prime}} \rrbracket_{L \mathbb{R}^{V}}=\llbracket \mathrm{A}_{G^{\prime}} \rightarrow \mathrm{A}_{G^{\prime}} \rrbracket_{L \mathbb{R}}$. Moreover, by lemma 42, Eq. (69) implies that $\|\mathscr{A}\|_{\text {sup }} \in J\left(\mathscr{V} \mathscr{A} \mathscr{V}^{-1}\right)$. Thus $\left\|\mathscr{V} \mathscr{A}^{-1}\right\|_{\text {sup }} \leq$ $\|\mathscr{A}\|_{\text {sup. }}$. Now, being $\mathscr{V}^{-1 \dagger}$ a UR, the equality $\left\|\mathscr{V} \mathscr{A} \mathscr{V}^{-1}\right\|_{\text {sup }}=\|\mathscr{A}\|_{\text {sup }}$ follows.

Lemma 44. Let $\mathscr{V}^{\dagger}$ be a UR. Then $\mathscr{V} \llbracket \mathrm{A}_{G^{\prime}} \rightarrow$ $\mathrm{A}_{G^{\prime}} \rrbracket_{Q \mathbb{R}^{\mathscr{V}}} \mathscr{V}^{-1}=\llbracket \mathrm{A}_{G^{\prime}} \rightarrow \mathrm{A}_{G^{\prime}} \rrbracket_{Q \mathbb{R}}$, and

$$
\lim _{n \rightarrow \infty} \mathscr{V} \mathscr{A}_{n} \mathscr{V}^{-1}=\mathscr{V}\left(\lim _{n \rightarrow \infty} \mathscr{A}_{n}\right) \mathscr{V}^{-1} .
$$

Proof. Since $\mathscr{V} \cdot \mathscr{V}^{-1}$ is isometric and surjective on the dense submanifolds $\llbracket \mathrm{A}_{G^{\prime}} \rightarrow \mathrm{A}_{G^{\prime}} \rrbracket_{L \mathbb{R}}$, by lemma 43 it maps Cauchy sequences to Cauchy sequences, and their equivalence classes to equivalence classes, thus it maps $\llbracket \mathrm{A}_{G^{\prime}} \rightarrow \mathrm{A}_{G^{\prime}} \rrbracket_{Q \mathbb{R}}$ to itself. Surjectivity follows from surjectivity on $\llbracket \mathrm{A}_{G^{\prime}} \rightarrow \mathrm{A}_{G^{\prime}} \rrbracket_{L \mathbb{R}}$. Eq. (70) can be independently checked applying both sides to arbitrary $a \in \llbracket \overline{\mathrm{A}}_{G^{\prime}} \rrbracket_{Q \mathbb{R}}$.

Lemma 45. Let $\mathscr{V}^{\dagger}$ be a UR. Then $\mathscr{V} \llbracket \mathrm{A}_{G^{\prime}} \rightarrow$ $\mathrm{A}_{G^{\prime}} \rrbracket_{Q 1} \mathscr{V}^{-1}=\llbracket \mathrm{A}_{G^{\prime}} \rightarrow \mathrm{A}_{G^{\prime}} \rrbracket_{Q 1}$.

Proof. Let $\left\{\mathscr{C}_{n}\right\}_{n \in \mathbb{N}}$ be a Cauchy sequence in $\llbracket \mathrm{A}_{G^{\prime}} \rightarrow \mathrm{A}_{G^{\prime}} \rrbracket_{L 1}$. Then by definition of UR and lemma 44 we have that $\left\{\mathscr{V} \mathscr{C}_{n} \mathscr{V}^{-1}\right\}_{n \in \mathbb{N}}$ is a Cauchy sequence in $\llbracket \mathrm{A}_{G^{\prime}} \rightarrow \mathrm{A}_{G^{\prime}} \rrbracket_{L 1}$, and its limit $\mathscr{V} \mathscr{C} \mathscr{V}^{-1}$ is in $\llbracket \mathrm{A}_{G^{\prime}} \rightarrow \mathrm{A}_{G^{\prime}} \rrbracket_{Q 1}$.
The last results finally allow us to prove that for a UR $\mathscr{V}^{\dagger}$, the map $\mathscr{V} \cdot \mathscr{V}^{-1}$ preserves the cones $\llbracket \mathrm{A}_{G^{\prime}} \rightarrow \mathrm{A}_{G^{\prime}} \rrbracket_{Q+}$.

Lemma 46. Let $\mathscr{V}^{\dagger}$ be a UR. Then $\mathscr{V} \llbracket \mathrm{A}_{G^{\prime}} \rightarrow$ $\mathrm{A}_{G^{\prime}} \rrbracket_{Q+} \mathscr{V}^{-1}=\llbracket \mathrm{A}_{G^{\prime}} \rightarrow \mathrm{A}_{G^{\prime}} \rrbracket_{Q+}$.

Proof. By lemmas 38 and 44, along with the definition of a UR, $\mathscr{V} \cdot \mathscr{V}^{-1}$ maps Cauchy sequences in $\llbracket \mathrm{A}_{G^{\prime}} \rightarrow \mathrm{A}_{G^{\prime}} \rrbracket_{L+}$ to Cauchy sequences in $\llbracket \mathrm{A}_{G^{\prime}} \rightarrow$ $\mathrm{A}_{G^{\prime}} \rrbracket_{L+}$, thus $\mathscr{V} \llbracket \mathrm{A}_{G^{\prime}} \rightarrow \mathrm{A}_{G^{\prime}} \rrbracket_{Q+} \mathscr{V}^{-1} \subseteq \llbracket \mathrm{A}_{G^{\prime}} \rightarrow$ $\mathrm{A}_{G^{\prime}} \rrbracket_{Q+}$. Since $\mathscr{V}^{-1 \dagger}$ is a UR, the thesis follows.

Next, we show that for a UR $\mathscr{V}^{\dagger}, \mathscr{V}^{\dagger} \llbracket \overline{\mathrm{A}}_{G^{\prime}} \rrbracket_{L \mathbb{R}}=$ $\llbracket \overline{\mathrm{A}}_{G^{\prime}} \rrbracket_{L \mathbb{R}}$.

Lemma 47. Let $\mathscr{V}^{\dagger}$ be a UR. Then $\mathscr{V}^{\dagger} \llbracket \overline{\mathrm{A}}_{G^{\prime}} \rrbracket_{L \mathbb{R}}=\llbracket \overline{\mathrm{A}}_{G^{\prime}} \rrbracket L \mathbb{R}$.

Proof. Let $a_{R} \in \llbracket \overline{\mathrm{A}}_{G^{\prime}} \rrbracket_{L \mathbb{R}}$. Then by proposition 14

$$
a_{R}=\mathscr{A}_{R}^{\dagger} e_{G^{\prime}},
$$

for some $\mathscr{A}_{R} \in \llbracket \mathrm{A}_{G^{\prime}} \rightarrow \mathrm{A}_{G^{\prime}} \rrbracket_{L \mathbb{R}}$. Now, we have

$$
\mathscr{V}^{\dagger} a_{R}=\mathscr{V}^{\dagger} \mathscr{A}_{R}^{\dagger} e_{G^{\prime}}=\mathscr{V}^{\dagger} \mathscr{A}_{R}^{\dagger} \mathscr{V}^{-1 \dagger} e_{G^{\prime}},
$$

and by lemma $43 \mathscr{V}^{\dagger} \mathscr{A}_{R}^{\dagger} \mathscr{V}^{-1 \dagger}=\mathscr{A}_{R^{\prime}}^{\prime \dagger}$ with $\mathscr{A}_{R^{\prime}}^{\prime} \in$ $\llbracket \mathrm{A}_{G^{\prime}} \rightarrow \mathrm{A}_{G^{\prime}} \rrbracket_{L \mathbb{R}}$. Then $\mathscr{V}^{\dagger} a_{R}=a_{R^{\prime}}^{\prime}$, with $R^{\prime} \in$ $\mathrm{R}^{\left(G^{\prime}\right)}$. As to surjectivity, it can be straightforwardly proved exploiting lemma 37 .

Finally, for a UR $\mathscr{V}^{\dagger}$, the map $\mathscr{V} \cdot \mathscr{V}^{-1}$ preserves the set of quasi-local transformations.

Lemma 48. Let $\mathscr{V}^{\dagger}$ be a UR. Then one has

$$
\begin{aligned}
& \mathscr{V} \llbracket \mathrm{A}_{G^{\prime}} \rightarrow \mathrm{A}_{G^{\prime}} \rrbracket_{Q^{\mathscr{V}}}=\llbracket \mathrm{A}_{G^{\prime}} \rightarrow \mathrm{A}_{G^{\prime}} \rrbracket_{Q}, \\
& \mathscr{V} \llbracket \mathrm{A}_{G^{\prime}} \rightarrow \mathrm{A}_{G^{\prime}} \rrbracket_{L} \mathscr{V}^{-1}=\llbracket \mathrm{A}_{G^{\prime}} \rightarrow \mathrm{A}_{G^{\prime}} \rrbracket_{L} .
\end{aligned}
$$

Proof. Let $\mathscr{A} \in \llbracket \mathrm{A}_{G^{\prime}} \rightarrow \mathrm{A}_{G^{\prime}} \rrbracket_{Q}$. Then $\|\mathscr{A}\|_{\text {sup }} \leq$ 1 , and by proposition 17 , there is $\mathscr{C} \in \llbracket \mathrm{A}_{G^{\prime}} \rightarrow$ $\mathrm{A}_{G^{\prime}} \rrbracket_{Q 1}$ such that

$$
\mathscr{C}-\mathscr{A} \succeq 0
$$

By lemma 46, $\mathscr{V} \mathscr{A} \mathscr{V}^{-1} \in \llbracket \mathrm{A}_{G^{\prime}} \rightarrow \mathrm{A}_{G^{\prime}} \rrbracket_{Q+}$. Moreover, by lemma $42, \mathscr{V} \mathscr{C}^{-1} \in \llbracket \mathrm{A}_{G^{\prime}} \rightarrow$ $\mathrm{A}_{G^{\prime}} \rrbracket_{Q 1}$. By the above observations, we have

$$
\begin{aligned}
& \mathscr{V} \mathscr{A} \mathscr{V}^{-1} \succeq 0, \\
& \mathscr{V} \mathscr{C} \mathscr{V}^{-1}-\mathscr{V} \mathscr{A} \mathscr{V}^{-1} \succeq 0 .
\end{aligned}
$$


Finally, by lemma 32, one has

$$
\mathscr{V} \mathscr{A}^{-1}, \mathscr{V} \mathscr{C} \mathscr{V}^{-1}-\mathscr{V} \mathscr{A} \mathscr{V}^{-1} \in \llbracket \mathrm{A}_{G^{\prime}} \rightarrow \mathrm{A}_{G^{\prime}} \rrbracket_{Q}
$$

The same argument holds for $\mathscr{A} \in \llbracket \mathrm{A}_{G^{\prime}} \rightarrow$ $\mathrm{A}_{G^{\prime}} \rrbracket_{L}$. Since $\mathscr{V}^{-1 \dagger}$ is a UR, surjectivity on both sets is proved.

Clearly, given a UR $\mathscr{V}^{\dagger}$, the image of the algebra of local transformations of $\mathrm{A}_{g}$ under $\mathscr{V} \cdot \mathscr{V}^{-1}$, i.e.

$$
\mathscr{V} \llbracket \mathrm{A}_{g}^{\left(G^{\prime}\right)} \rightarrow \mathrm{A}_{g}^{\left(G^{\prime}\right)} \rrbracket_{Q \mathbb{R}} \mathscr{V}^{-1} \subseteq \llbracket \mathrm{A}_{G}^{\left(G^{\prime}\right)} \rightarrow \mathrm{A}_{G}^{\left(G^{\prime}\right)} \rrbracket_{Q \mathbb{R}},
$$

is a subalgebra of $\llbracket \mathrm{A}_{G}^{\left(G^{\prime}\right)} \rightarrow \mathrm{A}_{G}^{\left(G^{\prime}\right)} \rrbracket_{Q \mathbb{R}}$ isomorphic to $\llbracket \mathrm{A}_{g}^{\left(G^{\prime}\right)} \rightarrow \mathrm{A}_{g}^{\left(G^{\prime}\right)} \rrbracket_{Q \mathbb{R}}$.

\subsection{Admissibility and local action}

As we have seen, independently of the nature of system $\mathrm{C}$, the map $\mathscr{V} \cdot \mathscr{V}^{-1}$ does more than mapping linear maps to linear maps: it sends transformations to transformations. This is an important result, as the definitions of automorphic family and UR are meant to embody necessary conditions for a family of maps to represent $\mathscr{V} \otimes \mathscr{I}_{\mathrm{C}}$. However, URs are not yet consistent with such a factorisation. The reason for this is that local discriminability in our theory is not assumed. Thus, while knowledge of the linear maps $\mathscr{V}_{\mathrm{C}}^{\dagger}$ is sufficient to determine the action of the UR on factorised effects $a_{G} \otimes b_{\mathrm{C}}$ and, by conjugation, on generalised transformations $\mathscr{A}_{G} \otimes \mathscr{I}_{\mathrm{C}}$, and such actions are consistent with a factorised action of the form $\mathscr{V} \otimes \mathscr{I}_{\mathrm{C}}$, still we do not have enough information to determine the action of $\mathscr{V}_{\mathrm{C}}^{\dagger}$ on those elements of $\llbracket \overline{\mathrm{A}}_{G} \overline{\mathrm{C}} \rrbracket_{Q \mathbb{R}}$ that lie outside the subspace $\llbracket \overline{\mathrm{A}}_{G} \rrbracket_{Q \mathbb{R}} \otimes \llbracket \overline{\mathrm{C}} \rrbracket_{Q \mathbb{R}}$ (and analogously for the conjugate action on transformations). For this purpose we introduce in this section the notion of admissibility.

We remind that $G^{\prime}$ stands for $G \cup h_{0}$, with $\mathrm{A}_{h_{0}} \cong \mathrm{C}$ for some system C. Let $R \in \mathrm{R}^{\left(G^{\prime}\right)}$ be a finite region. Since $\llbracket \overline{\mathrm{A}}_{R}^{\left(G^{\prime}\right)} \rrbracket_{Q \mathbb{R}}$ is isomorphic to $\llbracket \overline{\mathrm{A}}_{R} \rrbracket_{\mathbb{R}}$, whose size is $D_{R}:=D_{\mathrm{A}_{R}}$, we can find a basis $\left\{b_{j R}\right\}_{j=1}^{D_{R}} \subseteq \llbracket \overline{\mathrm{A}}_{R}^{\left(G^{\prime}\right)} \rrbracket_{Q \mathbb{R}}$. Thus, by lemma 47 , $\mathscr{V}^{\dagger} \llbracket \overline{\mathrm{A}}_{R}^{\left(G^{\prime}\right)} \rrbracket_{Q \mathbb{R}} \subseteq \llbracket \overline{\mathrm{A}}_{R^{\prime}}^{\left(G^{\prime}\right)} \rrbracket_{Q \mathbb{R}}$ for some finite region $R^{\prime} \in \mathrm{R}^{\left(G^{\prime}\right)}$, that can be obtained as

$$
R^{\prime}:=\bigcup_{j=1}^{D_{R}} R_{j}^{\prime}, \quad \mathscr{V}^{\dagger} b_{j R} \in \llbracket \overline{\mathrm{A}}_{R_{j}^{\prime}}^{\left(G^{\prime}\right)} \rrbracket_{Q \mathbb{R}} .
$$

This implies that, for every finite region $R \in$ $\mathrm{R}^{\left(G^{\prime}\right)}$, the UR $\mathscr{V}^{\dagger}$ induces a linear map $\mathscr{V}(R)^{\dagger}$ : $\llbracket \overline{\mathrm{A}}_{R} \rrbracket_{\mathbb{R}} \rightarrow \llbracket \overline{\mathrm{A}}_{R^{\prime}} \rrbracket_{\mathbb{R}}$. The following definition of admissible UR is now given in terms of the properties of the induced maps $\mathscr{V}(R)^{\dagger}$.

Definition 33. Let $\left(G, \mathrm{~A}, \mathscr{V}^{\dagger}\right)$ be a $U R$, and let $\mathscr{V}(R)^{\dagger}$ be the induced maps on finite regions $R \subseteq G^{\prime}$, for arbitrary choice of $\mathrm{A}_{h_{0}} \cong \mathrm{C}$. We call the $U R$ admissible if for every finite $S \in \mathrm{R}^{(G)}$ the family of maps $\mathscr{V}\left(S \cup h_{0}\right)^{\dagger}$ represents a transformation $\mathscr{V}_{S} \in \llbracket \mathrm{A}_{S^{\prime}} \rightarrow \mathrm{A}_{S} \rrbracket_{\mathbb{R}}$, i.e.

$$
\mathscr{V}\left(S \cup h_{0}\right)^{\dagger}=\mathscr{V}_{S}^{\dagger} \otimes \mathscr{I}_{\mathrm{C}}^{\dagger} .
$$

Consider now a partition $G=R \cup \bar{R}$, where $\bar{R}:=G \backslash R$. We will write $\mathrm{A}_{G}=\mathrm{A}_{R} \otimes \mathrm{A}_{\bar{R}}$. By definition, the space $\llbracket \overline{\mathrm{A}}_{G} \rrbracket_{Q \mathbb{R}}$ of quasi-local effects contains the spaces $\llbracket \overline{\mathrm{A}}_{R}^{(G)} \rrbracket_{Q \mathbb{R}}$ and $\llbracket \overline{\mathrm{A}}_{\bar{R}}^{(G)} \rrbracket_{Q \mathbb{R}}$ as closed subspaces. However, if the theory does not satisfy local discriminability, it is not true in general that $\llbracket \overline{\mathrm{A}}_{G} \rrbracket_{Q \mathbb{R}}=\llbracket \overline{\mathrm{A}}_{R}^{(G)} \rrbracket_{Q \mathbb{R}} \otimes \llbracket \overline{\mathrm{A}}_{\bar{R}}^{(G)} \rrbracket_{Q \mathbb{R}}$. This makes it difficult to define a UR of the form $\mathscr{V} \otimes \mathscr{W}$.

In the remainder of the section we close this gap. For this purpose, we start defining what it means, for a UR $\mathscr{V}^{\dagger}$ on $\mathrm{A}_{G}$, to act locally on $R$ in symbols $\mathscr{V}^{\dagger}=\mathscr{V}^{\prime \dagger} \otimes \mathscr{I}_{\bar{R}}^{\dagger}$. For our purpose, we then need to provide a suitable definition of the identity $\mathscr{V}^{\dagger}=\mathscr{V}^{\prime \dagger} \otimes \mathscr{I}_{\bar{R}}^{\dagger}$, which satisfies the necessary (but not sufficient) condition

$$
\left(\mathscr{V}^{\prime \dagger} \otimes \mathscr{I}_{\bar{R}}^{\dagger}\right)\left(a_{S} \otimes b_{T}\right)=\left(\mathscr{V}^{\prime \dagger} a_{S}\right) \otimes\left(b_{T}\right),
$$

for every $S, T \in \mathrm{R}^{(G)}$ such that $S \subseteq R$ and $T \subseteq \bar{R}$.

Definition 34. Let $\mathscr{V}^{\dagger}$ be a UR for $\mathrm{A}_{G}$. We say that $\mathscr{V}^{\dagger}$ acts locally on the region $R \subseteq G$ if for every $\mathrm{C}$ and every finite region $S \in \mathrm{R}^{\left(G^{\prime}\right)}$ with $G^{\prime}=G \cup h_{0}$ and $\mathrm{A}_{h_{0}} \cong \mathrm{C}$, the induced map $\mathscr{V}(S)^{\dagger}$ has the form

$$
\mathscr{V}^{\dagger}(S)=\mathscr{W}_{S \cap R}^{\dagger} \otimes \mathscr{I}_{S \backslash R}^{\dagger} .
$$

As a consequence of the above definition we now prove the following result, for which we do not provide the proof, that is straightforward.

Lemma 49. Let $\left(G, \mathrm{~A}, \mathscr{V}^{\dagger}\right)$ be a UR acting locally on $R$. Then

$$
\mathscr{V}^{\dagger}\left(a_{S} \otimes b_{T}\right)=\left(\mathscr{W}_{S}^{\dagger} a_{S}\right) \otimes b_{T},
$$

for $S \subseteq R$ and $T \subseteq \bar{R}$. In particular, $\mathscr{V}^{\dagger} \llbracket \overline{\mathrm{A}}_{R}^{\left(G^{\prime}\right)} \rrbracket_{Q \mathbb{R}}=\llbracket \overline{\mathrm{A}}_{R}^{\left(G^{\prime}\right)} \rrbracket_{Q \mathbb{R}}$, and $\mathscr{V}^{\dagger} b=b$ for every $b \in \llbracket \overline{\mathrm{A}}_{\bar{R}}^{\left(G^{\prime}\right)} \rrbracket_{Q \mathbb{R}}$. 
Lemma 50. Let $\left(G, \mathrm{~A}, \mathscr{V}^{\dagger}\right)$ be a UR acting locally on $R$. Then there exists a UR $\left(R, \mathrm{~A}, \mathscr{V}^{\prime \dagger}\right)$ such that $\mathscr{V}^{\dagger} \mathscr{J}_{R^{\prime}}^{-1 \dagger} a=\mathscr{J}_{R^{\prime}}^{-1 \dagger} \mathscr{V}^{\prime \dagger} a$ and $\mathscr{V}^{-1 \dagger} \mathscr{J}_{R^{\prime}}^{-1 \dagger} a=\mathscr{J}_{R^{\prime}}^{-1 \dagger} \mathscr{V}^{\prime-1 \dagger} a$ for every $a \in$ $\llbracket \overline{\mathrm{A}}_{R^{\prime}} \rrbracket_{Q \mathbb{R}}$, with $\mathrm{A}_{R^{\prime}}:=\mathrm{A}_{R} \mathrm{C}$.

Proof. Linearity and isometricity of the restriction $\mathscr{V}_{R^{\prime}}^{\dagger}$ of $\mathscr{V}^{\dagger}$ to $\llbracket \overline{\mathrm{A}}_{R^{\prime}}^{\left(G^{\prime}\right)} \rrbracket_{Q \mathbb{R}}$ are straightforward by lemma 49. Thus, also $\mathscr{V}^{\prime \dagger}:=\mathscr{J}_{R^{\prime}}^{\dagger} \mathscr{V}_{R^{\prime}}^{\dagger} \mathscr{J}_{R^{\prime}}^{-1 \dagger}$ is linear and isometric. Let now $\mathscr{A} \in \llbracket \mathrm{A}_{R^{\prime}} \rightarrow$ $\mathrm{A}_{R^{\prime}} \rrbracket_{Q}$. Then one has

$$
\begin{aligned}
\mathscr{V}^{\prime \dagger} \mathscr{A}^{\dagger} \mathscr{V}^{\prime-1 \dagger} & =\mathscr{J}_{R^{\prime}}^{\dagger} \mathscr{V}_{R^{\prime}}^{\dagger} \mathscr{J}_{R^{\prime}}^{-1 \dagger} \mathscr{A}^{\dagger} \mathscr{J}_{R^{\prime}}^{\dagger} \mathscr{V}_{R^{\prime}}^{-1 \dagger} \mathscr{J}_{R^{\prime}}^{-1 \dagger} \\
& =\mathscr{J}_{R^{\prime}}^{\dagger} \mathscr{V}_{R^{\prime}}^{\dagger} \mathscr{A}_{R^{\prime}}^{\dagger} \mathscr{V}_{R^{\prime}}^{-1 \dagger} \mathscr{J}_{R^{\prime}}^{-1 \dagger} \\
& =\mathscr{J}_{R^{\prime}}^{\dagger}\left(\mathscr{V}^{\dagger} \mathscr{A}_{R^{\prime}}^{\dagger} \mathscr{V}^{-1 \dagger}\right)_{R^{\prime}} \mathscr{J}_{R^{\prime}}^{-1 \dagger}
\end{aligned}
$$

where $\mathscr{A}_{R^{\prime}}^{\dagger}:=\mathscr{J}_{R^{\prime}}^{-1 \dagger} \mathscr{A}^{\dagger} \mathscr{J}_{R^{\prime}}^{\dagger}$. In the above chain of equalities we used the fact that, since $\mathscr{V}^{\dagger}$, $\mathscr{V}^{-1 \dagger}$ and $\mathscr{A}_{R^{\prime}}^{\dagger}$ preserve the space $\llbracket \overline{\mathrm{A}}_{R^{\prime}}^{\left(G^{\prime}\right)} \rrbracket_{Q \mathbb{R}}$, then also $\mathscr{V}^{\dagger} \mathscr{A}_{R^{\prime}}^{\dagger} \mathscr{V}^{-1 \dagger}$ does, and one can thus define its restriction $\left(\mathscr{V}^{\dagger} \mathscr{A}_{R^{\prime}}^{\dagger} \mathscr{V}^{-1 \dagger}\right)_{R^{\prime}}$. Finally, since $\mathscr{V}^{\dagger}$ is a UR, one has $\mathscr{V}^{\dagger} \mathscr{A}_{R^{\prime}}^{\dagger} \mathscr{V}^{-1 \dagger}=\mathscr{A}^{\prime \dagger}$ with $\mathscr{A}^{\prime} \in \llbracket \mathrm{A}_{R^{\prime}}^{\left(G^{\prime}\right)} \rightarrow \mathrm{A}_{R^{\prime}}^{\left(G^{\prime}\right)} \rrbracket_{Q} . \quad$ Thus, $\mathscr{V}^{\prime-1} \mathscr{A} \mathscr{V}^{\prime} \in$ $\llbracket \mathrm{A}_{R^{\prime}} \rightarrow \mathrm{A}_{R^{\prime}} \rrbracket_{Q} . \quad \mathrm{A}$ similar argument leads to the existence of $\mathscr{A}^{\prime \prime} \in \llbracket \mathrm{A}_{R^{\prime}} \rightarrow \mathrm{A}_{R^{\prime}} \rrbracket_{Q}$ such that $\mathscr{A}=\mathscr{V}^{\prime-1} \mathscr{A}^{\prime \prime} \mathscr{V}^{\prime}$. Now, let $\rho \in \llbracket \mathrm{A}_{G} \rrbracket$. Then $\rho$ defines a state $\rho_{\mid R^{\prime}}$ on $\llbracket \overline{\mathrm{A}}_{R^{\prime}} \rrbracket_{Q \mathbb{R}}$, by lemma 17 , with

$$
\left(a \mid \rho_{\mid R^{\prime}}\right)=\left(a_{R^{\prime}} \mid \rho\right)=\left(\mathscr{J}_{R^{\prime}}^{-1 \dagger} a \mid \rho\right),
$$

for every $a_{R^{\prime}} \in \llbracket \overline{\mathrm{A}}_{R^{\prime}}^{\left(G^{\prime}\right)} \rrbracket_{Q \mathbb{R}}$. Now, we have

$$
\begin{aligned}
& \left(a \mid[\hat{\mathscr{V}} \rho]_{\mid R^{\prime}}\right)=\left(\mathscr{J}_{R^{\prime}}^{-1 \dagger} a \mid \hat{\mathscr{V}} \rho\right)=\left(\mathscr{V}^{\dagger} \mathscr{J}_{R^{\prime}}^{-1 \dagger} a \mid \rho\right) \\
& \quad=\left(\mathscr{V}_{R^{\prime}}^{\dagger} \mathscr{J}_{R^{\prime}}^{-1 \dagger} a \mid \rho\right)=\left(\mathscr{J}_{R^{\prime}}^{-1 \dagger} \mathscr{J}_{R^{\prime}}^{\dagger} \mathscr{V}_{R^{\prime}}^{\dagger} \mathscr{J}_{R^{\prime}}^{-1 \dagger} a \mid \rho\right) \\
& \quad=\left(\mathscr{J}_{R^{\prime}}^{-1 \dagger} \mathscr{V}^{\prime \dagger} a \mid \rho\right)=\left(a \mid \hat{\mathscr{V}}^{\prime} \rho_{\mid R^{\prime}}\right) .
\end{aligned}
$$

We then showed that $[\hat{\mathscr{V}} \rho]_{\mid R^{\prime}}=\hat{\mathscr{V}}^{\prime}\left(\rho_{\mid R^{\prime}}\right)$. Moreover, since $\mathscr{V}$ preserves the set of states, $[\hat{\mathscr{V}} \rho]_{\mid R^{\prime}}$ is a state. Thus, $\hat{\mathscr{V}}^{\prime} \llbracket \mathrm{A}_{R^{\prime}} \rrbracket \subseteq \llbracket \mathrm{A}_{R^{\prime}} \rrbracket$. The same argument for $\mathscr{V}^{\prime-1}$ brings to the conclusion that $\hat{\mathscr{V}}^{\prime} \llbracket \mathrm{A}_{R^{\prime}} \rrbracket=\llbracket \mathrm{A}_{R^{\prime}} \rrbracket$. Thus, $\mathscr{V}^{\prime \dagger}$ is a UR for $\mathrm{A}_{R}$. Moreover

$$
\mathscr{V}^{\dagger} \mathscr{J}_{R^{\prime}}^{-1 \dagger} a=\mathscr{V}_{R^{\prime}}^{\dagger} \mathscr{J}_{R^{\prime}}^{-1 \dagger} a=\mathscr{J}_{R^{\prime}}^{-1 \dagger} \mathscr{V}^{\prime \dagger} a,
$$

and the same holds for $\mathscr{V}^{-1}$.

In the following, under the hypothesis of lemma 50, we will write $\mathscr{V}^{\dagger}=\mathscr{V}^{\prime \dagger} \otimes \mathscr{I}_{\bar{R}}^{\dagger}$.
Lemma 51. Let $\mathscr{V}^{\dagger}$ be a UR for $\llbracket \overline{\mathrm{A}}_{G} \rrbracket_{\mathbb{R}}$ that acts locally on $R$, and $\mathscr{W}^{\dagger}$ a UR that acts locally on $\bar{R}$. Then $\mathscr{V}^{\prime \dagger} \otimes \mathscr{W}^{\prime \dagger}:=\mathscr{V}^{\dagger} \mathscr{W}^{\dagger}=\mathscr{W}^{\dagger} \mathscr{V}^{\dagger}$.

We can now define a global update rule as follows.

Definition 35 (Global update rule). Let $\left(G, \mathrm{~A}, \mathscr{V}^{\dagger}\right)$ be a UR. We call $\left(G, \mathrm{~A}, \mathscr{V}^{\dagger}\right)$ a Global Update Rule (GUR), or global rule for short, if $\left(G, \mathrm{~A}, \mathscr{V}^{\dagger}\right)$ and $\left(G, \mathrm{~A}, \mathscr{V}^{-1 \dagger}\right)$ are admissible.

Remark 6. The set of GURs $\left(G, \mathrm{~A}, \mathscr{V}^{\dagger}\right)$ is a group. The proof of closure under composition and associativity are tedious but straightforward, and we omit them here. Moreover, given $\left(G, \mathrm{~A}, \mathscr{V}^{\dagger}\right)$ and $\left(F, \mathrm{~A}, \mathscr{W}^{\dagger}\right)$ one can define their parallel composition as $\left(G \cup F, \mathrm{~A}, \mathscr{V}^{\dagger} \otimes \mathscr{W}^{\dagger}\right)$. The analysis of properties of parallel composition is beyond the scope of the present work, and will be the subject of further studies.

We now provide an important example of GUR. Let $G=H \times\{0,1\}$, and let $H_{i}:=(H, i)$ for $i=0,1$. Let $a_{R} \in \llbracket \overline{\mathrm{A}}_{R}^{\left(G^{\prime}\right)} \rrbracket_{Q \mathbb{R}}$ with $\mathrm{A}_{h_{0}} \cong \mathrm{C}$. Let $R \cap G=\left(R_{0}, 0\right) \cup\left(R_{1}, 1\right)$. Then we define $\tilde{R}:=$ $R_{0} \cup R_{1} \subseteq H$, and $\bar{R}:=\tilde{R} \times\{0,1\} \cup R$. Clearly, $R \subseteq \bar{R}$. If $(h, i) \in \bar{R}$, then also $(h, i \oplus 1) \in \bar{R}$. Let then $\bar{a}_{\bar{R}}:=a_{R} \otimes e_{\bar{R} \backslash R}$.

Now, for every $T \in \overline{\mathrm{R}}^{(H)}$ let us define the GUR $\left(G, \mathrm{~A}, \mathscr{S}_{T}^{\dagger}\right)$ by setting

$$
\mathscr{S}_{T}^{\dagger}\left[a_{R}\right]:=\left[\left(\mathscr{S}_{\tilde{R} \cap T}^{\dagger} \bar{a}\right)_{\bar{R}}\right],
$$

where $\mathscr{S}_{X}^{\dagger}:=\bigotimes_{h \in X} \mathscr{S}_{h}^{\dagger}$ for $X \in \mathrm{R}^{(H)}$, and $\mathscr{S}_{h}$ is the map that swaps $\mathrm{A}_{(h, 0)}$ and $\mathrm{A}_{(h, 1)}$. It is straightforward to verify that, for every $T \in \overline{\mathrm{R}}^{(H)}$, $\mathscr{S}_{T}$ is a UR. Being defined in terms of induced maps, it is immediate to verify that $\mathscr{S}_{T}^{\dagger}$ admissible. Moreover, $\mathscr{S}_{T}$ is involutive, i.e. $\mathscr{S}_{T}^{-1}=\mathscr{S}_{T}$. Of particular interest for the following is the GUR $\left(G, \mathrm{~A}, \mathscr{S}_{H}^{\dagger}\right)$.

Notice that, for a finite disjoint partition $H=$ $\cup_{i=1}^{k} S_{k}$, we have $\mathscr{S}_{H}^{\dagger}=\bigotimes_{i=1}^{k} \mathscr{S}_{S_{k}}^{\dagger}$. Thus, by lemma 51, for every GUR $\left(G, \mathrm{~A}, \mathscr{V}^{\dagger}\right)$, and for every $i, j$, we have

$$
\begin{aligned}
& \mathscr{V}\left(\mathscr{S}_{S_{i} \cup S_{j}} \otimes \mathscr{I}_{H \backslash\left(S_{i} \cup S_{j}\right)}\right) \mathscr{V}^{-1} \\
& =\mathscr{V}\left(\mathscr{S}_{S_{i}} \otimes \mathscr{I}_{H \backslash S_{i}}\right) \mathscr{V}^{-1} \mathscr{V}\left(\mathscr{S}_{S_{j}} \otimes \mathscr{I}_{H \backslash S_{j}}\right) \mathscr{V}^{-1} \\
& =\mathscr{V}\left(\mathscr{S}_{S_{j}} \otimes \mathscr{I}_{H \backslash S_{j}}\right) \mathscr{V}^{-1} \mathscr{V}\left(\mathscr{S}_{S_{i}} \otimes \mathscr{I}_{H \backslash S_{i}}\right) \mathscr{V}^{-1}
\end{aligned}
$$

where $\mathscr{I}_{S}$ denotes $\mathscr{I}_{(S, 0)} \otimes \mathscr{I}_{(S, 1)}$. 
For $\mathscr{A} \in \llbracket \mathrm{A}_{R} \rightarrow \mathrm{A}_{R} \rrbracket$, it is easily verified that

$$
\begin{aligned}
& \mathscr{A}_{(R, 1)}=\mathscr{S}_{H}\left(\mathscr{A}_{(R, 0)}\right) \mathscr{S}_{H}=\mathscr{S}_{R}\left(\mathscr{A} \otimes \mathscr{I}_{R}\right) \mathscr{S}_{R}, \\
& \mathscr{A}_{(R, 0)}=\mathscr{S}_{H}\left(\mathscr{A}_{(R, 1)}\right) \mathscr{S}_{H}=\mathscr{S}_{R}\left(\mathscr{I}_{R} \otimes \mathscr{A}\right) \mathscr{S}_{R} .
\end{aligned}
$$

Finally, let us consider a $\operatorname{GUR}\left(G, \mathrm{~A}, \mathscr{U}^{\dagger} \otimes \mathscr{V}^{\dagger}\right)$. One can prove that $\mathscr{S}_{H}(\mathscr{U} \otimes \mathscr{V}) \mathscr{S}_{H}=\mathscr{V} \otimes \mathscr{U}$, by the following argument. Let us consider $a \in$ $\llbracket \overline{\mathrm{A}}_{R}^{\left(G^{\prime}\right)} \rrbracket_{Q \mathbb{R}}$. Then

$$
\begin{aligned}
\mathscr{S}_{H}^{\dagger} & (\mathscr{U} \otimes \mathscr{V})^{\dagger} \mathscr{S}_{H}^{\dagger} a_{R}=\mathscr{S}_{H}^{\dagger}(\mathscr{U} \otimes \mathscr{V})^{\dagger}\left(\mathscr{S}_{\tilde{R}}^{\dagger} a\right)_{\bar{R}} \\
& =\mathscr{S}_{H}^{\dagger}\left[\left(\mathscr{U}(\tilde{R}, 0)^{\dagger} \otimes \mathscr{V}(\tilde{R}, 1)^{\dagger}\right)\left(\mathscr{S}_{\tilde{R}}^{\dagger} a\right)\right]_{\bar{R}} \\
& =\left[\mathscr{S}_{\tilde{R}}^{\dagger}\left(\mathscr{U}(\tilde{R}, 0)^{\dagger} \otimes \mathscr{V}(\tilde{R}, 1)^{\dagger}\right)\left(\mathscr{S}_{\tilde{R}}^{\dagger} a\right)\right]_{\bar{R}} \\
& =\left[\mathscr{V}(\tilde{R}, 0)^{\dagger} \otimes \mathscr{U}(\tilde{R}, 1)^{\dagger} a\right]_{\bar{R}} \\
& =(\mathscr{V} \otimes \mathscr{U})^{\dagger} a_{R} .
\end{aligned}
$$

\subsection{Causal influence}

In the present subsection we define the notion of causal influence that establishes, given a GUR, whether the evolution allows interventions on one system to affect other systems after one step, or more generally how many steps are needed for such an influence to occur. We will often use the notation $\llbracket \mathrm{A}_{R}^{(G)} \mathrm{C} \rightarrow \mathrm{A}_{R}^{(G)} \mathrm{C} \rrbracket_{* *}$ to denote $\llbracket \mathrm{A}_{R}^{\left(G^{\prime}\right)} \rightarrow$ $\mathrm{A}_{R}^{\left(G^{\prime}\right)} \rrbracket_{* *}$, with $G^{\prime}=G \cup h_{0}$ and $\mathrm{A}_{h_{0}} \cong \mathrm{C}$. In the following we also adopt the convention that for $R \in \overline{\mathrm{R}}^{(G)}, \bar{R}$ denotes the complementary region $\bar{R}:=G \backslash R$. Notice that, consistently with the notation $R=g$, we will write $\bar{g}$ to denote $G \backslash g$.

Definition 36 (Causal influence). We say that, according to the global rule $\mathscr{V}$, system $g$ does not causally influence $g^{\prime}$ if for every external system C

$\mathscr{V} \llbracket \mathrm{A}_{g}^{(G)} \mathrm{C} \rightarrow \mathrm{A}_{g}^{(G)} \mathrm{C} \rrbracket_{Q \mathbb{R}} \mathscr{V}^{-1} \subseteq \llbracket \mathrm{A}_{\bar{g}^{\prime}}^{(G)} \mathrm{C} \rightarrow \mathrm{A}_{\bar{g}^{\prime}}^{(G)} \mathrm{C} \rrbracket_{Q \mathbb{R}}$.

On the other hand, we will say that according to the global rule $\mathscr{V}$ system $g$ causally influences system $g^{\prime}$, and write $g \longmapsto g^{\prime}$, in the opposite case, i.e. when

$\exists \mathrm{C}, \mathscr{F} \in \llbracket \mathrm{A}_{g}^{(G)} \mathrm{C} \rightarrow \mathrm{A}_{g}^{(G)} \mathrm{C} \rrbracket_{Q \mathbb{R}}:$

$\left(\mathscr{V} \otimes \mathscr{I}_{\mathrm{C}}\right) \mathscr{F}\left(\mathscr{V}^{-1} \otimes \mathscr{I}_{\mathrm{C}}\right) \notin \llbracket \mathrm{A}_{\bar{g}^{\prime}}^{(G)} \mathrm{C} \rightarrow \mathrm{A}_{\bar{g}^{\prime}}^{(G)} \mathrm{C} \rrbracket_{Q \mathbb{R}}$.

The above definition gets a clear meaning as a diagram: according to $\mathscr{V}$ there is causal influence from $g$ to $g^{\prime}$ if there exists $\mathscr{F} \in \llbracket \mathrm{A}_{g} \mathrm{C} \rightarrow \mathrm{A}_{g} \mathrm{C} \rrbracket$ such that

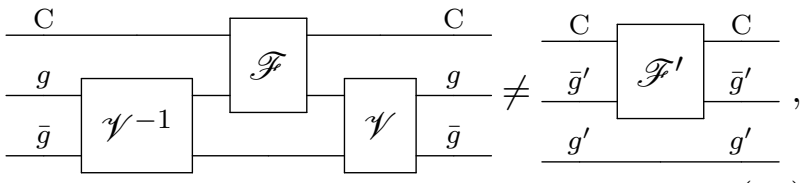

and even better in the form

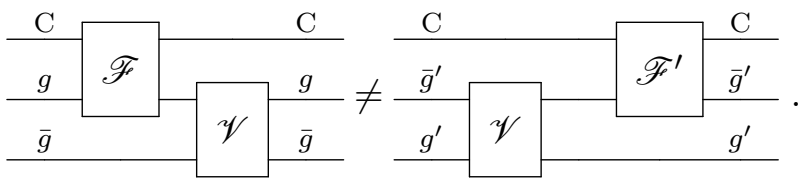

Definition 37. We define forward and backward $g$-neighbourhoods the sets $N_{g}^{+}:=\left\{g^{\prime} \mid g \longmapsto g^{\prime}\right\}$ and $N_{g}^{-}:=\left\{g^{\prime} \mid g^{\prime} \longmapsto g\right\}$, respectively.

In terms of diagrams, the forward $g$ neighbourhood $\mathrm{A}_{N_{g}^{+}}$of $g$ can be understood as the smallest region $K=\bigotimes_{k \in K} A_{g_{k}^{\prime}}$ such that for every $k \in K$ there exists $\mathrm{C}$ and $\mathscr{F} \in \llbracket \mathrm{A}_{g}^{(G)} \mathrm{C} \rightarrow$ $\mathrm{A}_{g}^{(G)} \mathrm{C} \rrbracket_{Q \mathbb{R}}$ such that
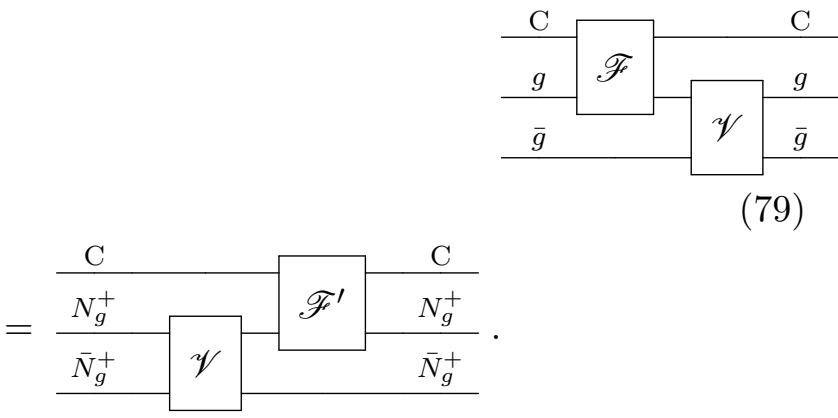

with $\mathscr{F}^{\prime}$ acting non trivially on $g_{k}^{\prime}$, namely $\mathscr{F}^{\prime} \notin$ $\llbracket \mathrm{A}_{\bar{g}_{k}^{\prime}}^{(G)} \mathrm{C} \rightarrow \mathrm{A}_{\bar{g}_{k}^{\prime}}^{(G)} \mathrm{C} \rrbracket_{Q \mathbb{R}}$. Notice that $g$ may or may not belong to $N_{g}^{+}$.

Analogously to the definition of $N_{g}^{ \pm}$, one can give a definition of $N_{R}^{ \pm}$for every finite region $R \subseteq$ $G$.

Definition 38. We say that the system $g^{\prime}$ belongs to the forward neighbourhood of $R$, and write $g^{\prime} \in$ $N_{R}^{+}$, if

$\exists \mathrm{C}, \mathscr{F} \in \llbracket \mathrm{A}_{R}^{(G)} \mathrm{C} \rightarrow \mathrm{A}_{R}^{(G)} \mathrm{C} \rrbracket_{Q \mathbb{R}}:$

$\left(\mathscr{V} \otimes \mathscr{I}_{\mathrm{C}}\right) \mathscr{F}\left(\mathscr{V}^{-1} \otimes \mathscr{I}_{\mathrm{C}}\right) \notin \llbracket \mathrm{A}_{\bar{g}^{\prime}}^{(G)} \mathrm{C} \rightarrow \mathrm{A}_{\bar{g}^{\prime}}^{(G)} \mathrm{C} \rrbracket_{Q \mathbb{R}}$.

The backward neighbourhood $N_{R}^{-}$of $R$ is defined as

$$
N_{R}^{-}:=\left\{g^{\prime} \in G \mid N_{g^{\prime}}^{+} \cap R \neq \emptyset\right\} .
$$


Clearly, one has

$$
N_{R}^{+} \supseteq \bigcup_{g \in R} N_{g}^{+} .
$$

We will prove that the latter is actually an equality in the next section.

One might define causal influence without invoking any external system $\mathrm{C}$, as follows.

Definition 39 (Individual influence). We say that the system $g$ does not individually influence $g^{\prime}$ if

$$
\begin{gathered}
\mathscr{V} \llbracket \mathrm{A}_{g}^{(G)} \rightarrow \mathrm{A}_{g}^{(G)} \rrbracket_{Q \mathbb{R}^{\mathscr{V}}} \mathscr{V}^{-1} \\
\quad \subseteq \llbracket \mathrm{A}_{\bar{g}^{\prime}}^{(G)} \rightarrow \mathrm{A}_{\bar{g}^{\prime}}^{(G)} \rrbracket_{Q \mathbb{R}} .
\end{gathered}
$$

On the other hand, we will say that according to the global rule $\mathscr{V}$ system $g$ individually influences system $g^{\prime}$, and write $g \longmapsto g g^{\prime}$, in the opposite case, i.e. when

$$
\begin{aligned}
& \exists \mathscr{F} \in\left[\mathrm{A}_{g}^{(G)} \rightarrow \mathrm{A}_{g}^{(G)}\right]_{Q \mathbb{R}}: \\
& \mathscr{V} \mathscr{F} \mathscr{V}^{-1} \notin \llbracket \mathrm{A}_{\bar{g}^{\prime}}^{(G)} \rightarrow \mathrm{A}_{\bar{g}^{\prime}}^{(G)} \rrbracket_{Q \mathbb{R}} .
\end{aligned}
$$

The individual forward neighbourhood of $g$ is

$$
N_{g}^{+}(g):=\left\{g^{\prime} \in G \mid g \longmapsto_{g} g^{\prime}\right\} .
$$

A second definition that can be given without referring to external systems $\mathrm{C}$ is the following.

Definition 40 (Cooperative influence). We say that the region $S$ does not cooperatively influence $g^{\prime}$ if

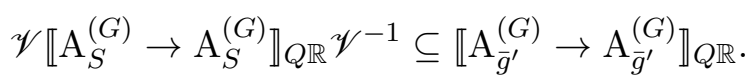

In the opposite case we will write $S \longmapsto_{S} g^{\prime}$. The cooperative forward neighbourhood of a region $S$ is defined as

$$
N_{S}^{+}(S):=\left\{g^{\prime} \in G \mid S \longmapsto_{S} g^{\prime}\right\} .
$$

Notice now that in a theory without local discriminability it may happen that a system $g$ does not individually influence $g^{\prime}$ but it does cooperatively, namely one can have $g^{\prime} \in N_{S}^{+}(S)$, but $g^{\prime} \notin N_{g}^{+}(g)$ for any $g \in S$. In other words, $N_{S}^{+}(S) \supset \bigcup_{h \in S} N_{h}^{+}(h)$. We call this phenomenon non-local activation of causal influence. If such a phenomenon occurs, however, it can be hard to establish which of the "cooperating" systems $h \in S$ is responsible for causal influence on $g^{\prime} \in N_{S}^{+}(S)$. This is the reason why we prefer the former definition of causal influence, which provides also this piece of information.

Given a GUR $\left(G, \mathrm{~A}, \mathscr{V}^{\dagger}\right)$, we can now construct a graph $\Gamma(G, E)$ with vertices $g \in G$ and edges $E:=\left\{\left(g, g^{\prime}\right) \in G \times G \mid g \longmapsto g^{\prime}\right\}$. We call such a graph the graph of causal influence of the GUR $\mathscr{V}^{\dagger}$. In the next section we will prove that, if we denote by $N_{g}^{ \pm *}$ the neighbourhoods for the GUR $\mathscr{V}^{-1}$, the following relations hold

$$
N_{f}^{ \pm}=N_{f}^{\mp *} .
$$

We also define the local perspective present $P_{R}^{+}$ of $R \in \mathrm{R}^{(G)}$ as the set $P_{R}^{+}:=N_{N_{R}^{+}}^{-} \supseteq R$, namely the set of systems that can causally influence the one-step future $N_{R}^{+}$of $R$. Similarly one can define the local retrospective present of $R$ as $P_{R}^{-}:=N_{N_{R}^{-}}^{+} \supseteq R$, namely the set of systems that can be influenced by the one-step past $N_{R}^{-}$ of $R$. In general, $P_{R}^{+} \neq P_{R}^{-}$. We will also need to refer to the sets $P_{R}^{+*}:=N_{N_{R}^{+}}^{+*}$.

\subsubsection{Relation with signalling}

The term influence that we used above is precisely defined in Eq. (78). In the present subsection we will make a clear point that this definition is stronger than the notion of signalling that is customary in the literature on quantum information theory $[52,53,54]$ or in general operational probabilistic theories [15]. In that context, a channel $\mathscr{C} \in \llbracket \mathrm{AB} \rightarrow \mathrm{A}^{\prime} \mathrm{B}^{\prime} \rrbracket$ is called nonsignalling (or semicausal) from $\mathrm{B}$ to $\mathrm{A}^{\prime}$ if there exists $\mathscr{F} \in \llbracket \mathrm{A} \rightarrow \mathrm{A}^{\prime} \rrbracket$ such that

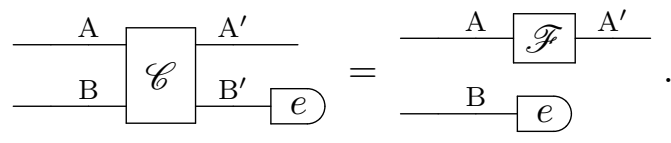

We will now show that, under the hypothesis that $N_{g}^{+*}$ is finite for every $g \in G$, the condition of Eq. 79 , in a suitably defined sense, implies that of Eq. (83). Let us consider the state $\rho \in \llbracket \mathrm{A}_{G} \rrbracket_{\mathbb{R}}$. Then $\left(a_{S} \mid \hat{\mathscr{A}}_{T} \rho\right):=\left(a_{S \cup T}^{\prime} \mid \rho\right)=\left(\mathscr{A}_{T}^{\dagger} a_{S} \mid \rho\right)$. In the case where $a_{S}=e_{G},\left(\mathscr{A}_{T}^{\dagger} e_{G} \mid \rho\right)=\left(b_{T} \mid \rho\right)$, where $b=\mathscr{A}^{\dagger} e_{T}$. Let us now consider $(\hat{\mathscr{V}} \rho)_{\mid R}$, which by definition is the state in $\llbracket \mathrm{A}_{R} \rrbracket_{\mathbb{R}}$ such that, for every $a \in \llbracket \overline{\mathrm{A}}_{R} \rrbracket_{\mathbb{R}}$

$$
\left(a \mid[\hat{\mathscr{V}} \rho]_{\mid R}\right)=\left(\mathscr{J}_{R}^{\dagger} a \mid \hat{\mathscr{V}} \rho\right)=\left(a_{R} \mid \hat{\mathscr{V}} \rho\right)
$$


Now, by lemma 31, one has

$$
\begin{aligned}
\left(a_{R} \mid \hat{\mathscr{V}} \rho\right) & =\left(e_{G} \mid \hat{\mathscr{A}}_{R}^{\hat{\mathscr{V}}} \rho\right) \\
& =\left(e_{G} \mid \hat{\mathscr{V}}^{-1} \hat{\mathscr{A}}_{R} \hat{\mathscr{V}} \rho\right) \\
& =\left(e_{G} \mid \hat{\mathscr{V}}_{N_{N_{R}}^{+*}}^{\prime} \rho\right) \\
& =\left(e_{G} \mid \hat{\mathscr{A}}_{N_{R}^{+*}}^{\prime} \rho\right) \\
& =\left(b_{N_{R}^{+*}} \mid \rho\right)=\left(b \mid \rho_{\mid N_{R}^{+*}}\right) .
\end{aligned}
$$

In order to determine $[\hat{\mathscr{V}} \rho]_{\mid R}$ it is sufficient to

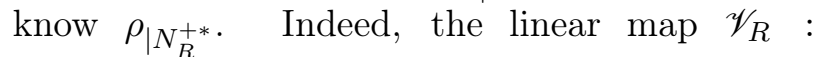
$\llbracket \mathrm{A}_{N_{R}^{+*}} \rrbracket \mathbb{R} \rightarrow \llbracket \mathrm{A}_{R} \rrbracket \mathbb{R}$ gives

$$
(\hat{\mathscr{V}} \rho)_{\mid R}=\mathscr{V}_{R}\left(\rho_{\mid N_{R}^{+*}}\right) .
$$

Diagrammatically, the equality can be recast, with a slight abuse of notation, as in Eq. 83, as follows

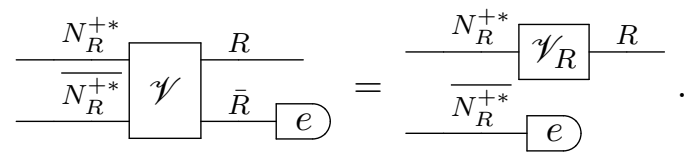

Notice that, by the no-restriction hypothesis, for an admissible $\mathscr{V}$ the map $\mathscr{V}_{R}$ is linear and admissible, and is thus a transformation in $\llbracket \mathrm{A}_{N_{R}^{+*}}^{(G)} \rightarrow$ $\mathrm{A}_{R}^{(G)} \rrbracket$. Moreover,

$$
\mathscr{V}^{\dagger} a_{R}=\left(\mathscr{V}_{R}^{\dagger} a\right)_{N_{R}^{+*}}
$$

Finally, notice that by definition $\mathscr{V}_{R}^{\dagger} e_{R}=e_{N_{R}^{+*}}$, namely $\mathscr{V}_{R} \in \llbracket \mathrm{A}_{N_{R}^{+*}}^{(G)} \rightarrow \mathrm{A}_{R}^{(G)} \rrbracket_{1}$. The argument can be repeated for the case where $N_{g}^{+*}$ is not finite, as long as $N_{g}^{+*} \subset G$.

We remark that in the case of Quantum or Fermionic cellular automata, the condition for no causal influence is equivalent to no-signalling, while in the case of Classical cellular automata no causal influence is strictly stronger than nosignalling. In a different context, a difference between the two notions was pointed out in Ref. [55]

\subsection{Block decomposition}

Let us now consider the GUR $\left(G, \mathrm{~A}, \mathscr{W}^{\dagger}\right)$ where we set $G=H \times\{0,1\}$, and $\mathscr{W}=\mathscr{V}_{H_{0}} \otimes \mathscr{V}_{H_{1}}^{-1}$, for an arbitrary GUR $\left(H, \mathrm{~A}, \mathscr{V}^{\dagger}\right), H_{i}$ denoting a shorthand for $(H, i)$. We observe that

$\mathscr{W}=\mathscr{V}_{H_{0}} \otimes \mathscr{V}_{H_{1}}^{-1}=\left(\mathscr{V}_{H_{0}} \otimes \mathscr{I}_{H_{1}}\right) \mathscr{S}_{H}\left(\mathscr{V}_{H_{0}}^{-1} \otimes \mathscr{I}_{H_{1}}\right) \mathscr{S}_{H}$
Then, by Eq. (76), given $R \subseteq \mathrm{A}_{G}$ and $a \in$ $\llbracket \overline{\mathrm{A}}_{R}^{(G)} \overline{\mathrm{C}} \rrbracket_{Q \mathbb{R}}$, with $R \cap G=\left(R_{0}, 0\right) \cup\left(R_{1}, 1\right)$,

$$
\mathscr{W}^{\dagger} a_{R \mathrm{C}}=\left(\mathscr{W}^{\dagger} a\right)_{R^{\prime} \mathrm{C}}
$$

$=\left[\mathscr{S}_{H}^{\dagger}\left(\mathscr{V}_{H_{0}}^{-1} \otimes \mathscr{I}_{H_{1}}\right)^{\dagger} \mathscr{S}_{H}^{\dagger}\left(\mathscr{V}_{H_{0}} \otimes \mathscr{I}_{H_{1}}\right)^{\dagger}\right] a_{R \mathrm{C}}$

$=\left[\mathscr{S}_{H}^{\dagger}\left(\mathscr{V}_{H_{0}}^{-1} \otimes \mathscr{I}_{H_{1}}\right)^{\dagger} \mathscr{S}_{H}^{\dagger}\right] a_{R^{\prime \prime} \mathrm{C}}^{\prime}$

$=\left[\mathscr{S}_{H}^{\dagger}\left(\mathscr{V}_{H_{0}}^{-1} \otimes \mathscr{I}_{H_{1}}\right)^{\dagger} \mathscr{S}_{N_{R_{0}}^{+*} \cup R_{1}}^{\dagger}\right] a_{R^{\prime \prime} \mathrm{C}}^{\prime}$

$=\left[\mathscr{S}_{H}^{\dagger}\left(\mathscr{V}_{H_{0}}^{-1} \otimes \mathscr{I}_{H_{1}}\right)^{\dagger} \mathscr{S}_{N_{R_{0}}^{+*} \cup R_{1}}^{\dagger}\left(\mathscr{V}_{H_{0}} \otimes \mathscr{I}_{H_{1}}\right)^{\dagger}\right] a_{R \mathrm{C}}$,

where $\quad a^{\prime}:=\left(\mathscr{V}_{H_{0}} \otimes \mathscr{I}_{H_{1}}\right)^{\dagger} a, \quad R^{\prime} \quad:=$ $\left(N_{R_{0}}^{+*}, 0\right) \cup\left(N_{R_{1}}^{+}, 1\right)$, and $R^{\prime \prime}:=\left(N_{R_{0}}^{+*}, 0\right) \cup\left(R_{1}, 1\right)$. Now, defining for $S \in \overline{\mathrm{R}}^{(H)}$

$$
\begin{aligned}
\mathscr{S}_{S}^{\prime} & :=\prod_{h \in S} \mathscr{S}_{h}^{\prime}, \\
\mathscr{S}_{h}^{\prime} & :=\left(\mathscr{V}_{H_{0}} \otimes \mathscr{I}_{H_{1}}\right) \mathscr{S}_{h}\left(\mathscr{V}_{H_{0}}^{-1} \otimes \mathscr{I}_{H_{1}}\right),
\end{aligned}
$$

thanks to eq. (77) we have

$$
\begin{aligned}
\left(\mathscr{V}_{H_{0}}^{-1}\right. & \left.\otimes \mathscr{I}_{H_{1}}\right)^{\dagger} \mathscr{S}_{N_{R_{0}}^{+*} \cup R_{1}}^{\dagger}\left(\mathscr{V}_{H_{0}} \otimes \mathscr{I}_{H_{1}}\right)^{\dagger} \\
& =\mathscr{S}_{N_{R_{0}}^{+*} \cup R_{1}}^{\prime \dagger}
\end{aligned}
$$

We remark that, since $\left(\mathscr{V}_{H_{0}} \otimes \mathscr{I}_{H_{1}}\right) \cdot\left(\mathscr{V}_{H_{0}}^{-1} \otimes \mathscr{I}_{H_{1}}\right)$ is an automorphism of $\llbracket \mathrm{A}_{G} \rightarrow \mathrm{A}_{G} \rrbracket_{Q \mathbb{R}}$, one has

$$
\mathscr{S}_{f}^{\prime} \mathscr{S}_{g}^{\prime}=\mathscr{S}_{g}^{\prime} \mathscr{S}_{f}^{\prime}, \quad \forall f, g \in H .
$$

Thus, the product $\prod_{h \in N_{R}^{+*}} \mathscr{S}_{h}^{\dagger}$ in Eq. (86) is well defined. Notice also that, by definition

$$
\mathscr{S}_{h}^{\prime} \mathscr{S}_{h}^{\prime}=\mathscr{I}_{G}
$$

Since $\mathscr{S}_{h}^{\prime} \in \llbracket \mathrm{A}_{\left(N_{h}^{+}, 0\right) \cup(h, 1)}^{(G)} \rightarrow \mathrm{A}_{\left(N_{h}^{+}, 0\right) \cup(h, 1)}^{(G)} \rrbracket_{Q 1}$ (see Fig. 1), one has that $\mathscr{S}_{N_{R_{0}}^{+*} \cup R_{1}}^{\prime} \in \llbracket \mathrm{A}_{S}^{(G)} \rightarrow$ $\mathrm{A}_{S}^{(G)} \rrbracket_{Q 1}$, with

$$
S:=\left(N_{N_{R_{0}}^{+*}}^{+} \cup N_{R_{1}}^{+}, 0\right) \cup\left(N_{R_{0}}^{+*} \cup R_{1}, 1\right) .
$$

Then we can write

$$
\begin{aligned}
\mathscr{W}^{\dagger} a_{R \mathrm{C}} & =\mathscr{S}_{H}^{\dagger} \mathscr{S}_{N_{R_{0}}^{+*} \cup R_{1}}^{\prime \dagger} a_{R \mathrm{C}} \\
& =\mathscr{S}_{N_{N_{R_{0}}^{+*}}^{\dagger} \cup N_{R_{1}}^{+} \cup N_{R_{0}}^{+*} \cup R_{1}} \mathscr{S}_{N_{R_{0}}^{+*} \cup R_{1}} a_{R \mathrm{C}} .
\end{aligned}
$$

However, due to the structure of $\mathscr{W}^{\dagger}$, one actually has

$$
\left(\mathscr{S}_{N_{R_{0}}^{+*} \cup R_{1}}^{\prime} \otimes \mathscr{I}_{\mathrm{C}}\right)^{\dagger} a_{R \mathrm{C}}=a_{\left(N_{R_{1}}^{+}, 0\right) \cup\left(N_{R_{0}}^{+*}, 1\right) \mathrm{C}}^{\prime \prime}
$$



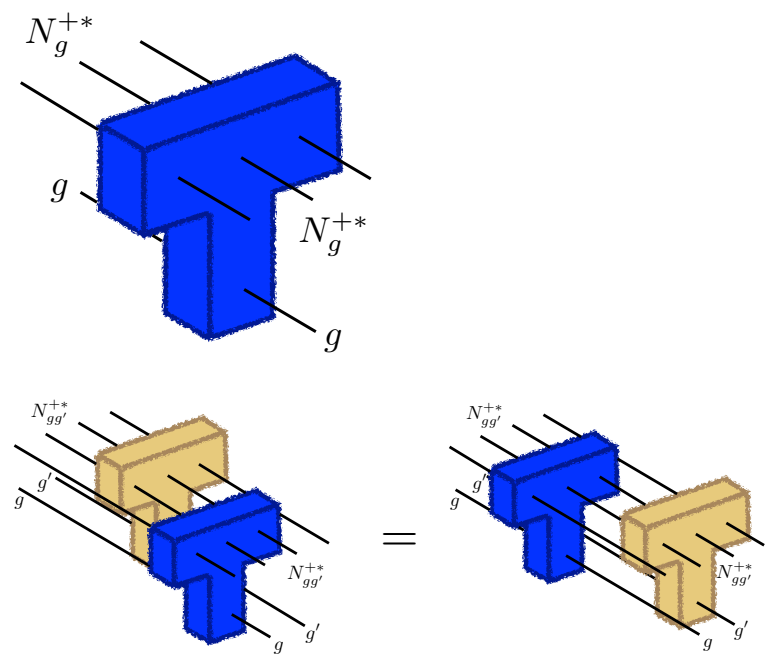

Figure 1: In the top picture we provide a graphical representation of the transformation $\mathscr{S}_{g}^{\prime}$, with input wires on the top left and output on the bottom right. In the bottom picture we provide a graphical illustration of the identity $\mathscr{S}_{g}^{\prime} \mathscr{S}_{g^{\prime}}^{\prime}=\mathscr{S}_{g^{\prime}}^{\prime} \mathscr{S}_{g}^{\prime}$.

and finally this implies that

$$
\mathscr{W}^{\dagger} a_{R \mathrm{C}}=\left(\mathscr{S}_{N_{R_{0}}^{+*} \cup N_{R_{1}}^{+}}^{\dagger} \mathscr{S}_{N_{R_{0}}^{+\dagger} \cup R_{1}} \otimes \mathscr{I}_{\mathrm{C}}^{\dagger}\right) a_{R \mathrm{C}}
$$

We now observe that

$$
\mathscr{W}^{-1}=\mathscr{V}_{H_{0}}^{-1} \otimes \mathscr{V}_{H_{1}}=\mathscr{S}_{H} \mathscr{W}_{\mathscr{S}_{H}},
$$

and thus, by Eq. (90), it is

$$
\begin{aligned}
\mathscr{W}^{-1 \dagger} a_{R \mathrm{C}} & =\left(\mathscr{S}_{N_{R_{1}}^{+*} \cup R_{0}}^{\prime} \otimes \mathscr{I}_{\mathrm{C}}\right)^{\dagger} a_{R^{\prime} \mathrm{C}}^{\prime} \\
& =\left(\mathscr{S}_{N_{R_{1}}^{\prime+} \cup R_{0}}^{\dagger} \mathscr{S}_{R_{0} \cup R_{1}}^{\dagger} \otimes \mathscr{I}_{\mathrm{C}}^{\dagger}\right) a_{R \mathrm{C}},
\end{aligned}
$$

where $a_{R^{\prime} \mathrm{C}}^{\prime}=\left[\mathscr{S}^{\dagger} a\right]_{\left(R_{1}, 0\right) \cup\left(R_{0}, 1\right) \mathrm{C} \text {. Let us then }}$ consider $R_{1}=\emptyset$. In this case for $\left[a_{(R, 0) \mathrm{C}}\right] \in$ $\llbracket \overline{\mathrm{A}}_{(R, 0)}^{(G)} \overline{\mathrm{C}} \rrbracket$, being $R=(R, 0) \cup(\emptyset, 1)$, it holds that

$$
\begin{aligned}
& {\left[\left(\mathscr{V}^{\dagger} a\right)_{\left(N_{R}^{+*}, 0\right) \mathrm{C}}\right]=\mathscr{S}_{N_{R}^{+*}}^{\dagger} \mathscr{S}_{N_{R}^{+*}}^{\prime \dagger} \otimes \mathscr{I}_{\mathrm{C}}^{\dagger}\left[a_{(R, 0) \mathrm{C}}\right],} \\
& {\left[\left(\mathscr{V}^{-1}{ }^{\dagger} a\right)_{\left(N_{R}^{+}, 0\right) \mathrm{C}}\right]=\mathscr{S}_{R}^{\prime \dagger} \mathscr{S}_{R}^{\dagger} \otimes \mathscr{I}_{\mathrm{C}}^{\dagger}\left[a_{(R, 0) \mathrm{C}}\right] .}
\end{aligned}
$$

In view of the above results, we now consider the maps $\mathscr{V} \mathscr{A}^{-1}$ for $\mathscr{A} \in \llbracket \mathrm{A}_{(R, 0)} \mathrm{C} \rightarrow \mathrm{A}_{(R, 0)} \mathrm{C} \rrbracket_{\mathbb{R}}$, with $R \in \mathrm{R}^{(H)}$. First of all, we observe that

$$
\left(\mathscr{V} \mathscr{A} \mathscr{V}^{-1}\right) \otimes \mathscr{I}_{H_{1}}=\mathscr{W}\left(\mathscr{A} \otimes \mathscr{I}_{H_{1}}\right) \mathscr{W}^{-1}
$$

is a local transformation in $\in \llbracket \mathrm{A}_{\left(N_{R}^{+}, 0\right)}^{(G)} \mathrm{C} \rightarrow$ $\mathrm{A}_{\left(N_{R}^{+}, 0\right)}^{(G)} \mathrm{C} \rrbracket_{Q \mathbb{R}}$. Let then $a \in \llbracket \overline{\mathrm{A}}_{\tilde{S}}^{(G)} \overline{\mathrm{C}} \rrbracket_{\mathbb{R}}$ for $S \in$
$\mathrm{R}^{(H)}$. We have the following (see Appendix C)

$$
\begin{aligned}
& {\left[\left(\mathscr{V} \mathscr{A}^{-1}\right) \otimes \mathscr{I}_{H_{1}}\right]^{\dagger} a_{\tilde{S} \mathrm{C}} } \\
= & {\left[\mathscr{W}\left(\mathscr{A} \otimes \mathscr{I}_{H_{1}}\right) \mathscr{W}^{-1}\right]^{\dagger} a_{\tilde{S} \mathrm{C}} } \\
= & \left(\mathscr{S}_{R}^{\prime \dagger} \mathscr{S}_{R}^{\dagger} \otimes \mathscr{I}_{\mathrm{C}}^{\dagger}\right)\left(\mathscr{A} \otimes \mathscr{I}_{H_{0}}\right)^{\dagger}\left(\mathscr{S}_{R}^{\dagger} \mathscr{S}_{R}^{\prime \dagger} \otimes \mathscr{I}_{\mathrm{C}}^{\dagger}\right) a_{\tilde{S} \mathrm{C}} .
\end{aligned}
$$

Finally, since this holds for every $a$, we have that for $\mathscr{A} \in \llbracket \mathrm{A}_{(R, 0)} \mathrm{C} \rightarrow \mathrm{A}_{(R, 0)} \mathrm{C} \rrbracket_{\mathbb{R}}$

$$
\begin{aligned}
& \left(\mathscr{V}_{\mathscr{A}} \mathscr{V}^{-1}\right) \otimes \mathscr{I}_{H_{1}} \\
= & \left(\mathscr{S}_{R}^{\prime} \otimes \mathscr{I}_{\mathrm{C}}\right)\left(\mathscr{I}_{H_{0}} \otimes \mathscr{A}\right)\left(\mathscr{S}_{R}^{\prime} \otimes \mathscr{I}_{\mathrm{C}}\right) .
\end{aligned}
$$

The above relation, considering Eq. (86), shows that

$$
N_{R}^{+} \subseteq \bigcup_{g \in R} N_{g}^{+}
$$

Along with Eq. (81) the latter proves that actually

$$
N_{R}^{+}=\bigcup_{g \in R} N_{g}^{+}
$$

Analogously, since $N_{R}^{-}=\left\{g^{\prime} \mid \exists g \in R, g \in N_{g^{\prime}}^{+}\right\}$, it is

$$
N_{R}^{-}=\left\{g^{\prime} \mid \exists g \in R, g^{\prime} \in N_{g}^{-}\right\}=\bigcup_{g \in R} N_{g}^{-} .
$$

Let us finally consider the maps $\left(\mathscr{V}^{-1} \mathscr{B} \mathscr{V}\right)$ for $\mathscr{B} \in \llbracket \mathrm{A}_{(R, 1)} \mathrm{C} \rightarrow \mathrm{A}_{(R, 1)} \mathrm{C} \rrbracket_{\mathbb{R}}$, with $R \in \mathrm{R}^{(H)}$. In this case, using Eq. (89) and manipulating Eq. (94) we obtain

$$
\begin{aligned}
& \mathscr{I}_{H_{0}} \otimes\left(\mathscr{V}^{-1} \mathscr{B} \mathscr{V}\right) \\
& \quad=\left(\mathscr{S}_{N_{R}^{+*}}^{\prime} \otimes \mathscr{I}_{\mathrm{C}}^{\dagger}\right)\left(\mathscr{B} \otimes \mathscr{I}_{H_{1}}\right)\left(\mathscr{S}_{N_{R}^{+*}}^{\prime} \otimes \mathscr{I}_{\mathrm{C}}\right) .
\end{aligned}
$$

Since the $\mathscr{S}_{h}^{\prime}$ 's that do not commute with $\mathscr{B} \otimes$ $\mathscr{I}_{H_{1}}$ in Eq. (97) correspond to $h \in N_{g}^{-}$for some $g \in R$, namely $h \in N_{R}^{-}$, we can conclude that

$$
N_{R}^{+*} \subseteq N_{R}^{-}
$$

This observation is sufficient to prove that $N_{R}^{ \pm *}=$ $N_{R}^{\mp}$, as anticipated in the previous section.

Lemma 52. Let $\left(G, \mathrm{~A}, \mathscr{V}^{\dagger}\right)$ be a GUR. Then

$$
N_{R}^{ \pm *}=N_{R}^{\mp} .
$$


Proof. We remind that $g \in N_{f}^{ \pm}$iff $f \in N_{g}^{\mp}$. Now, considering Eq. (98) for $R=g$ we have that

$$
g^{\prime} \in N_{g}^{-*} \Leftrightarrow g \in N_{g^{\prime}}^{+*} \Rightarrow g \in N_{g^{\prime}}^{-} \Leftrightarrow g^{\prime} \in N_{g}^{+} .
$$

This implies that $N_{g}^{-*} \subseteq N_{g}^{+}$. Exchanging $\mathscr{V}$ and $\mathscr{V}^{-1}$, we can conclude that $N_{g}^{+*} \subseteq N_{g}^{-} \subseteq$ $N_{g}^{+*}$, i.e. $N_{g}^{+*}=N_{g}^{-}$. The thesis follows invoking Eqs. (95) and (96), which then give us

$$
N_{R}^{-}=N_{R}^{+*} .
$$

In the remainder we will then use $N_{R}^{ \pm}$and $P_{R}^{ \pm}$, and abandon the symbols $N_{R}^{ \pm *}$ and $P_{R}^{ \pm *}$.

We observe that the block decomposition of $\mathscr{V} \otimes \mathscr{V}^{-1}$ that we achieved through the block transformations $\mathscr{S}_{g}^{\prime}$ is a generalisation of a result that was proved for quantum cellular automata in Ref. [32]. Local rules of other kinds are used in the literature, with nice properties that the present block decomposition lacks, such as composability (see e.g. Ref [56]). However, for later purposes the block form of Eq. (86) is particularly suitable in our case, to cope with update rules in theories without local discriminability.

\section{Homogeneity}

The principle of homogeneity, as it is usually formulated in physics, regards equivalence of points in space-time. Here, however, space-time is not the background for occurrence of physical events, on the contrary it emerges as a convenient description of relations between information processing events. We then provide a definition of homogeneity for a global update rule, that regards the way in which different systems are treated by the evolution. In particular, roughly speaking, every system must be treated in the same way by the GUR.

It would be easy to state the homogeneity principle if we knew in advance that the set $G$ has some geometric structure that is invariant under the action of a group - technically speaking, if $G$ were a symmetric space - so that we could define pairs of homologous regions as those mapped into one another by some group element. In this case, we could provide a criterion for the evolution to treat every system in the same way, consisting in the existence of some representation of the group of symmetries of the set $G$ that connects the evolution of every two homologous regions. The group in this case would provide the two following ingredients that are needed for the definition of homogeneity: i) a notion of "running the same test on different regions", and ii) a criterion for equivalence of two homologous regions provided by the requirement that the events of the same test in the two regions have equal probabilities, if the regions are prepared in the same state.

However, we are in a situation where the structure of $G$ must emerge from the evolution given by the global rule $\mathscr{V}$. The detailed idea is thus to turn the above argument on its head, and define two regions to be homologous if they are "treated in the same way" by $\mathscr{V}$. The precise construction is the following.

First of all, in order for the dynamics to treat two regions in the same way, the two regions need to have the same structure, in such a way that we can make sense of "performing the same range of tests in the two different regions". This concept is captured by the extension of the notion of operational equivalence (see definition 2) to regions. Intuitively, two operationally equivalent regions must correspond to operationally equivalent systems. However, they also need to"have the same structure", where the term structure refers to the fact that a system $\mathrm{A}_{R}$ associated with a region $R$ is decomposed in a preferred way into subsystems $\mathrm{A}_{g_{i}}$ corresponding to the cells that compose the region $R=\left\{g_{1}, g_{2}, \ldots, g_{k}\right\}$. The notion of operational equivalence of regions must then also capture the idea that two equivalent regions admit equivalent decompositions.

The definition is thus recursive, starting from elementary regions $R=\{g\}$, and then defining equivalence of $R_{1}$ and $R_{2}$ as the equivalence of any strict subregion $S_{1} \subset R_{1}$ with a strict subregion $S_{2} \subset R_{2}$, and viceversa.

We recall Remark 5, namely that for the remainder every elementary region $g \in G$ corresponds to some non-trivial system, $\mathrm{A}_{g} \nsucceq \mathrm{I}$.

Definition 41. Two elementary regions $g$ and $h$ are operationally equivalent if $\mathrm{A}_{g} \cong \mathrm{A}_{h}$. Two finite regions $R_{1}, R_{2} \subseteq G$ are operationally equivalent if $\mathrm{A}_{R_{1}} \simeq \mathrm{A}_{R_{2}}$, and for every $S_{1} \subset R_{1}$ there exists $S_{2} \subset R_{2}$, and viceversa for every $S_{2} \subset R_{2}$ there exists $S_{1} \subset R_{1}$, such that $\mathrm{A}_{S_{1}}$ and $\mathrm{A}_{S_{2}}$ are operationally equivalent.

Two operationally equivalent regions have the same structure, namely they can be decomposed 
into pairwise equivalent subsystems, as we now prove.

Lemma 53. An elementary region $R_{1}=\{g\}$ cannot be operationally equivalent to a nonelementary one $R_{2}=\left\{h_{1}, \ldots, h_{k}\right\}$.

Proof. The statement is trivial, since $R_{1}=\left\{g_{1}\right\}$ does not have proper subregions, while any nonelementary region $R_{2}$ does, so operational equivalence cannot hold.

Lemma 54. The regions $R_{1}$ and $R_{2}$ are operationally equivalent if and only if $R_{1}=$ $\left\{g_{1}, g_{2}, \ldots, g_{k}\right\}, R_{2}=\left\{h_{1}, h_{2}, \ldots h_{k}\right\}$, and $\mathrm{A}_{g_{i}} \cong$ $\mathrm{A}_{h_{i}}$.

Proof. The condition is clearly sufficient, as one can immediately realise by considering the reversible transformation $\mathscr{T}_{S} \in \llbracket \mathrm{A}_{S_{1}} \rightarrow \mathrm{A}_{S_{2}} \rrbracket_{1}$ defined as $\mathscr{T}_{S}:=\bigotimes_{g_{j} \in S_{1}} \mathscr{T}_{j}$, with $\mathscr{T}_{j} \in \llbracket \mathrm{A}_{g_{j}} \rightarrow$ $\mathrm{A}_{h_{j}} \rrbracket_{1}$ reversible for every $j=1, \ldots, k$. We now prove that the condition is necessary. Let $R_{1}$ and $R_{2}$ be operationally equivalent. By definition, and by lemma 53, for every $g_{l} \in R_{1}$ there is $h_{m} \in R_{2}$ such that $\mathrm{A}_{g_{l}} \cong \mathrm{A}_{h_{m}}$, and viceversa. We now introduce the equivalence relation

$$
g_{i} \sim g_{j} \Leftrightarrow \mathrm{A}_{g_{i}} \cong \mathrm{A}_{g_{j}} .
$$

We can then partition $R_{1}$ and $R_{2}$ into equivalence classes $\left[g_{i}\right]$ and $\left[h_{j}\right]$, respectively, obtaining $R_{1}=$ $\bigcup_{g_{i_{l}}}\left[g_{i_{l}}\right]$ and $R_{2}=\bigcup_{h_{i_{l}}}\left[h_{i_{l}}\right]$. Let us start considering the case where $R_{1}$ contains a unique equivalence class: $R_{1}=\left[g_{1}\right]$. Then $R_{2}$ must contain at least one element $h_{1}$ with $\mathrm{A}_{h_{1}} \cong \mathrm{A}_{g_{1}}$. Moreover, $R_{2}$ cannot contain $h_{0}$ such that $h_{0} \nsim h_{1}$, because $h_{0}$ must be equivalent to an elementary subregion of $R_{1}$, by lemma 53. Then, $R_{2}=\left[h_{1}\right]$. Finally, since $\mathrm{A}_{g_{1}}^{\otimes h} \cong \mathrm{A}_{R_{2}} \cong \mathrm{A}_{R_{1}} \cong \mathrm{A}_{g_{1}}^{\otimes k}$, by lemma 2 it must be $h=k$. In the general case where $R_{1}$ contains more than one equivalence class, one can simply consider the subregions $S_{1_{l}}:=\left[g_{i_{l}}\right]$. Clearly, for each $S_{1_{l}}$ there must be $S_{2_{l}}=\left[h_{j_{l}}\right]$, with $\left|S_{2_{l}}\right|=\left|S_{1_{l}}\right|$.

Remark 7. Notice that, as a consequence of the above result, two operationally equivalent regions $R_{1}, R_{2} \in \mathrm{R}^{(G)}$ have the same cardinality $\left|R_{1}\right|=$ $\left|R_{2}\right|$.

Given two operationally equivalent regions $R_{1}, R_{2} \subseteq G$, we now have a way of defining the notion of "running the same test", that resorts to the identification of a canonical isomorphism of the two regions.
Definition 42. Given two operationally equivalent regions $R_{1}=\left\{g_{1}, g_{2}, \ldots, g_{k}\right\}, R_{2}=$ $\left\{h_{1}, h_{2}, \ldots, h_{k}\right\} \in \mathrm{R}^{(G)}$, let us choose a special reversible transformation $\mathscr{U} \in \llbracket \mathrm{A}_{R_{1}} \rightarrow \mathrm{A}_{R_{2}} \rrbracket$ such that $\mathscr{U}=\bigotimes_{i=1}^{k} \mathscr{U}_{i}$, where $\mathscr{U}_{i} \in \llbracket \mathrm{A}_{g_{i}} \rightarrow \mathrm{A}_{h_{i}} \rrbracket$ is reversible for every $i$. We then say that $R_{1}$ is operationally equivalent to $R_{2}$ through $\mathscr{U}$, and that $\left\{\mathscr{B}_{l}^{(2)}\right\}_{l=1}^{n} \subseteq \llbracket \mathrm{A}_{R_{2}} \mathrm{C} \rightarrow \mathrm{A}_{R_{2}} \mathrm{C} \rrbracket$ represents the same test as $\left\{\mathscr{B}_{l}^{(1)}\right\}_{l=1}^{n} \subseteq \llbracket \mathrm{A}_{R_{1}} \mathrm{C} \rightarrow \mathrm{A}_{R_{1}} \mathrm{C} \rrbracket$ if for every $1 \leq l \leq n$ one has

$$
\mathscr{B}_{l}^{(2)}=\left(\mathscr{U} \otimes \mathscr{I}_{\mathrm{C}}\right) \mathscr{B}_{l}^{(1)}\left(\mathscr{U}^{-1} \otimes \mathscr{I}_{\mathrm{C}}\right) .
$$

In order to discriminate the way in which two systems $\mathrm{A}_{g_{1}}$ and $\mathrm{A}_{g_{2}}$ evolve, one needs to perform a testing-scheme, consisting of a stimulustest $\left\{\mathscr{A}_{k}^{(x)}\right\}_{k=1}^{m} \subseteq \llbracket \mathrm{A}_{R_{x}} \mathrm{C} \rightarrow \mathrm{A}_{R_{x}} \mathrm{C} \rrbracket_{Q}$ on the region $R_{x} \ni g_{x}$, possibly involving an ancillary system $\mathrm{C}$, and, after the evolution step $\mathscr{V}$, a controltest $\left\{\mathscr{B}_{l}^{(x)}\right\}_{l=1}^{n} \subseteq \llbracket \mathrm{A}_{N_{R_{x}}^{+}} \mathrm{C} \rightarrow \mathrm{A}_{N_{R_{x}}^{+}} \mathrm{C} \rrbracket_{Q}$ on the region $N_{R_{x}}^{+}$(where $x \in\{1,2\}$ ) and the ancilla $\mathrm{C}$, in order to detect a "response".

Two operationally equivalent regions are homologous if there is a GUR $\mathscr{T}$ that interchanges them while preserving equivalence of local tests of finite regions. In rigorous words, we have the following definition.

Definition 43 (Homologous regions). Let $\left(G, \mathrm{~A}, \mathscr{V}^{\dagger}\right)$ be a GUR, and let $R_{1}, R_{2} \in \mathrm{R}^{(G)}$ be operationally equivalent regions through $\mathscr{T}_{i} \in$ $\llbracket \mathrm{A}_{R_{1}} \rightarrow \mathrm{A}_{R_{2}} \rrbracket$, such that $N_{R_{1}}^{+}$and $N_{R_{2}}^{+}$are operationally equivalent through $\mathscr{T}_{o} \in \llbracket \mathrm{A}_{R_{1}^{\prime}} \rightarrow$ $\mathrm{A}_{R_{2}^{\prime}} \rrbracket$. We say that $R_{2}$ is homologous to $R_{1}$ if there exists an admissible $G U R\left(G, \mathrm{~A}, \mathscr{T}^{\dagger}\right)$, such that for all schemes $\left(\left\{\mathscr{A}_{k}^{(i)}\right\}_{k=1}^{m},\left\{\mathscr{B}_{l}^{(i)}\right\}_{l=1}^{n}\right) \subseteq$ $\llbracket \mathrm{A}_{R_{i}} \mathrm{C} \rightarrow \mathrm{A}_{R_{i}} \mathrm{C} \rrbracket_{Q} \times \llbracket \mathrm{A}_{N_{R_{i}}^{+}} \mathrm{C} \rightarrow \mathrm{A}_{N_{R_{i}}^{+}} \mathrm{C} \rrbracket_{Q}$ representing the same pair of tests for $i=1,2$, one has $\mathscr{B}_{l}^{(1)} \mathscr{V} \mathscr{A}_{k}^{(1)}=\mathscr{T}^{-1} \mathscr{B}_{l}^{(2)} \mathscr{V} \mathscr{A}_{k}^{(2)} \mathscr{T}$. We denote this relation as $R_{2} \bowtie R_{1}$.

We remark that, while the relation $\bowtie$ defined above clearly depends on $\mathscr{V}$, we will generally not make this fact explicit in the notation, as we will always consider contexts where a given $\mathscr{V}$ is considered, and there will not be room for ambiguity. On the other hand, when we want to specify the GUR $\mathscr{T}$ that makes $R_{1}$ homologous to $R_{2}$, we write $R_{2} \bowtie \mathscr{T} R_{1}$.

Lemma 55. If $R_{2} \bowtie_{\mathscr{T}} R_{1}$ then $R_{1} \bowtie_{\mathscr{T}-1} R_{2}$. 
Proof. For every scheme $\left(\left\{\mathscr{A}_{k}^{(i)}\right\}_{k=1}^{m},\left\{\mathscr{B}_{l}^{(i)}\right\}_{l=1}^{n}\right)$ with $\mathscr{A}_{k}^{(1)}=\left(\mathscr{T}_{i}^{-1} \otimes \mathscr{I}_{\mathrm{C}}\right) \mathscr{A}_{k}^{(2)}\left(\mathscr{T}_{i} \otimes \mathscr{I}_{\mathrm{C}}\right)$ and $\mathscr{B}_{l}^{(1)}=\left(\mathscr{T}_{o}^{-1} \otimes \mathscr{I}_{\mathrm{C}}\right) \mathscr{B}_{l}^{(2)}\left(\mathscr{T}_{o} \otimes \mathscr{I}_{\mathrm{C}}\right)$, one has $\mathscr{B}_{l}^{(1)} \mathscr{V} \mathscr{A}_{k}^{(1)}=\mathscr{T}^{-1} \mathscr{B}_{l}^{(2)} \mathscr{V} \mathscr{A}_{k}^{(2)} \mathscr{T}$. Now, inverting $\mathscr{T}$ on the right and $\mathscr{T}^{-1}$ on the left, one obtains $\mathscr{B}_{l}^{(2)} \mathscr{V} \mathscr{A}_{k}^{(2)}=\mathscr{T} \mathscr{B}_{l}^{(1)} \mathscr{V}_{\mathscr{A}_{k}^{(1)}}^{(1)} \mathscr{T}^{-1}$.

Lemma 56. Let $\mathscr{V}$ be a global rule, and let the region $R_{2}$ be homologous to $R_{1}$ through $\mathscr{T}$. Then $\mathscr{T}^{-1} \mathscr{V} \mathscr{T}=\mathscr{V}$

Proof. It is sufficient to consider the special case where the test scheme is $\left(\left\{\mathscr{A}_{0}^{(1)}\right\},\left\{\mathscr{B}_{0}^{(1)}\right\}\right)$, with $\mathscr{A}_{0}^{(1)}=\mathscr{I}_{R_{1} \mathrm{C}}$ and $\mathscr{B}_{0}^{(1)}=\mathscr{I}_{N_{R_{1}}^{+} \mathrm{C}}$. Then, we have $\mathscr{A}_{0}^{(2)}=\mathscr{I}_{R_{2} \mathrm{C}}, \mathscr{B}_{0}^{(2)}=\mathscr{I}_{N_{R_{2}}^{+} \mathrm{C}}$, and $\mathscr{V}=$ $\mathscr{T}^{-1} \mathscr{V} \mathscr{T}$.

Lemma 57. Let $\mathscr{V}$ be a global rule, and let $R_{2} \bowtie$ $R_{1}$ for some suitable $\mathscr{T}$. Then

$$
\begin{aligned}
& \mathscr{T} \llbracket \mathrm{A}_{R_{1}}^{(G)} \mathrm{C} \rightarrow \mathrm{A}_{R_{1}}^{(G)} \mathrm{C} \rrbracket \mathscr{T}^{-1}=\llbracket \mathrm{A}_{R_{2}}^{(G)} \mathrm{C} \rightarrow \mathrm{A}_{R_{2}}^{(G)} \mathrm{C} \rrbracket, \\
& \mathscr{T} \llbracket \mathrm{A}_{N_{R_{1}}^{+}}^{(G)} \mathrm{C} \rightarrow \mathrm{A}_{N_{R_{1}}^{+}}^{(G)} \mathrm{C} \rrbracket \mathscr{T}^{-1}=\llbracket \mathrm{A}_{N_{R_{2}}^{+}}^{(G)} \mathrm{C} \rightarrow \mathrm{A}_{N_{R_{2}}^{+}}^{(G)} \mathrm{C} \rrbracket .
\end{aligned}
$$

Proof. Let us choose the test scheme as $\left(\left\{\mathscr{A}_{k}^{(1)}\right\}_{k=1}^{m},\left\{\mathscr{B}_{0}^{(1)}\right\}\right)$, with $\mathscr{B}_{0}^{(1)}=\mathscr{I}_{R_{1}^{\prime} \mathrm{C}}$. Then, we have $\mathscr{B}_{0}^{(2)}=\mathscr{I}_{R_{2}^{\prime} \mathrm{C}}$, and

$$
\begin{aligned}
& \mathscr{V} \mathscr{A}_{k}^{(1)}=\mathscr{T}^{-1} \mathscr{V} \mathscr{A}_{k}^{(2)} \mathscr{T} \\
= & \mathscr{T}^{-1} \mathscr{V}\left(\mathscr{T}_{i} \otimes \mathscr{I}_{\mathrm{C}}\right) \mathscr{A}_{k}^{(1)}\left(\mathscr{T}_{i}^{-1} \otimes \mathscr{I}_{\mathrm{C}}\right) \mathscr{T} \\
= & \mathscr{T}^{-1} \mathscr{V} \mathscr{T}^{-1}\left(\mathscr{T}_{i} \otimes \mathscr{I}_{\mathrm{C}}\right) \mathscr{\mathscr { A }}_{k}^{(1)}\left(\mathscr{T}_{i}^{-1} \otimes \mathscr{I}_{\mathrm{C}}\right) \mathscr{T} \\
= & \mathscr{V} \mathscr{T}^{-1}\left(\mathscr{T}_{i} \otimes \mathscr{I}_{\mathrm{C}}\right) \mathscr{A}_{k}^{(1)}\left(\mathscr{T}_{i}^{-1} \otimes \mathscr{I}_{\mathrm{C}}\right) \mathscr{T},
\end{aligned}
$$

where in the last equality we used the result of lemma 56. If we now invert $\mathscr{V}$ to the left on both sides, we obtain

$$
\mathscr{A}_{k}^{(1)}=\mathscr{T}^{-1}\left(\mathscr{T}_{i} \otimes \mathscr{I}_{\mathrm{C}}\right) \mathscr{A}_{k}^{(1)}\left(\mathscr{T}_{i}^{-1} \otimes \mathscr{I}_{\mathrm{C}}\right) \mathscr{T},
$$

and finally

$$
\begin{aligned}
& \mathscr{T}_{k}^{(1)} \mathscr{T}^{-1} \\
= & \left(\mathscr{T}_{i} \otimes \mathscr{I}_{\mathrm{C}}\right) \mathscr{A}_{k}^{(1)}\left(\mathscr{T}_{i}^{-1} \otimes \mathscr{I}_{\mathrm{C}}\right)=\mathscr{A}_{k}^{(2)} .
\end{aligned}
$$

A similar argument leads us to conclude that

$$
\begin{aligned}
& \mathscr{T} \mathscr{B}_{k}^{(1)} \mathscr{T}^{-1} \\
= & \left(\mathscr{T}_{o} \otimes \mathscr{I}_{\mathrm{C}}\right) \mathscr{B}_{k}^{(1)}\left(\mathscr{T}_{o}^{-1} \otimes \mathscr{I}_{\mathrm{C}}\right)=\mathscr{B}_{k}^{(2)} .
\end{aligned}
$$

We remark that, straightforwardly,

$$
\left(R_{2} \bowtie R_{1}\right) \Rightarrow\left(\mathrm{A}_{R_{1}} \cong \mathrm{A}_{R_{2}}\right) .
$$

Now, the discrimination of systems $g_{1}$ and $g_{2}$ is successful if there exists a region $R_{1} \ni g_{1}$ such that for any operationally equivalent region $R_{2} \ni$ $g_{2}$, the region $R_{2}$ is not homologous to $R_{1}$. In precise terms, we have the following definition.

Definition 44 (Absolute discrimination). The evolution given by the global rule $\mathscr{V}$ discriminates two systems $g_{1}, g_{2} \in G$ if for any GUR $\mathscr{T}$ there exists a region $R_{1}$, containing $g_{1}$, for which there is no region $R_{2}$, containing $g_{2}$, that is homologous to $R_{1}$ through $\mathscr{T}$, with $g_{1} \bowtie \mathscr{T} g_{2}$.

In simple words, the evolution given by the global rule $\mathscr{V}$ allows for discrimination of the two systems $g_{1}, g_{2} \in G$ if there exists a test that gives different probabilities depending on whether it is applied at $g_{1}$ or at $g_{2}$, or if the tests that one can run on $R_{1}$ cannot be performed on any region $R_{2}$ containing $g_{2}$, or viceversa.

Now, the notion of discrimination of systems $g_{1}$ and $g_{2}$ with respect to a third system $e$ is given as follows.

Definition 45 (Relative discrimination). The evolution $\mathscr{V}$ discriminates two systems $g_{1}, g_{2} \in G$ relatively to a reference system $e$, with $e \in G$, if for every $\mathscr{T}$ there exists a region $R_{1}$, containing $\left\{g_{1}, e\right\}$, for which there is no region $R_{2}$, containing $\left\{g_{2}, e\right\}$, homologous to $R_{1}$ through $\mathscr{T}$ with $e \bowtie \mathscr{T} e$ and $g_{1} \bowtie \mathscr{T} g_{2}$.

We can now require the evolution described by a global rule to be homogeneous by a precise statement.

Principle 1 (Homogeneity). The global rule $\mathscr{V}$ discriminates any two systems $g_{1} \neq g_{2} \in G$, relatively to an arbitrary reference system $e \in G$, but not absolutely.

Before providing a convenient restatement of the principle, we analyse some of its aspects and consequences. In the first place, the principle says that if we do not choose a reference element $e \in G$, every two systems $g_{1}$ and $g_{2}$ cannot be discriminated. If we take the negation of definition 44 , we obtain a straightforward consequence of homogeneity. 
Lemma 58. Let $\mathscr{V}$ satisfy the homogeneity principle 1. For every pair $g_{1}, g_{2} \in G$, there exists $\mathscr{T}$ such that for every choice of region $R_{1} \ni$ $g_{1}$, there exists an operationally equivalent region $R_{2} \ni g_{2}$ through $\mathscr{T}_{i} \in \llbracket \mathrm{A}_{R_{1}} \rightarrow \mathrm{A}_{R_{2}} \rrbracket$ such that also $N_{R_{1}}^{+}$and $N_{R_{2}}^{+}$are operationally equivalent through $\mathscr{T}_{o} \in \llbracket \mathrm{A}_{R_{1}^{\prime}} \rightarrow \mathrm{A}_{R_{2}^{\prime}} \rrbracket$, and for all schemes $\left(\left\{\mathscr{A}_{k}^{(i)}\right\}_{k=1}^{m},\left\{\mathscr{B}_{l}^{(i)}\right\}_{l=1}^{n}\right)$ representing the same tests for $i=1,2$, one has $\mathscr{B}_{l}^{(1)} \mathscr{V} \mathscr{A}_{k}^{(1)}=$ $\mathscr{T} \mathscr{B}_{l}^{(2)} \mathscr{V} \mathscr{A}_{k}^{(2)} \mathscr{T}^{-1}$.

Proof. The statement follows negating definition 44.

We can now draw a first non trivial consequence from the statement of principle 1 .

Lemma 59. For a homogeneous GUR $\left(G, \mathrm{~A}, \mathscr{V}^{\dagger}\right)$, all local systems $\mathrm{A}_{g}$ for $g \in G$ are operationally equivalent.

Proof. Let us consider two elements $g_{1}, g_{2} \in G$. As a consequence of the homogeneity principle 1 , for the special choice $R_{1}=\left\{g_{1}\right\}$, there exists $R_{2} \ni g_{2}$ such that $R_{2} \bowtie\left\{g_{1}\right\}$ for a suitable $\mathscr{T}$. Now, by lemma 54 , it must be $\left|R_{2}\right|=\left|\left\{g_{1}\right\}\right|=1$, i.e. $R_{2}=\left\{g_{2}\right\}$. Thus, we have

$$
\mathrm{A}_{g_{2}} \cong \mathrm{A}_{g_{1}}
$$

Corollary 15. If the region $R_{2}$ containing $g_{2}$ is homologous to $\left\{g_{1}\right\}$ through $\mathscr{T}$, then $R_{2}=\left\{g_{2}\right\}$, and

$$
\mathscr{T}^{-1} \llbracket \mathrm{A}_{g_{1}}^{(G)} \mathrm{C} \rightarrow \mathrm{A}_{g_{1}}^{(G)} \mathrm{C} \rrbracket \mathscr{T}=\llbracket \mathrm{A}_{g_{2}}^{(G)} \mathrm{C} \rightarrow \mathrm{A}_{g_{2}}^{(G)} \mathrm{C} \rrbracket .
$$

Proof. By the proof of lemma 59 one has that $R_{2}=g_{2}$. The thesis then follows from lemma 57 .

The second result that we prove is a technical lemma that will play a crucial role in the following results.

Lemma 60. Let $\left(G, \mathrm{~A}, \mathscr{V}^{\dagger}\right)$ satisfy the homogeneity requirement. Then for every region $R$ one has $\mathrm{A}_{R}=\bigotimes_{x \in R} \mathrm{~A}_{g_{x}} \cong \mathrm{A}_{0}^{\otimes|R|}$, where $\mathrm{A}_{0} \cong \mathrm{A}_{g}$ for every $g \in G$.

Proof. By lemma 59 we know that for every $g \in$ $G$ one has $\mathrm{A}_{g} \cong \mathrm{A}_{0}$. The remaining part of the statement thus follows straightforwardly.
Lemma 61. Let $\mathscr{V}$ be non trivial and satisfy the homogeneity requirement, and $R_{2} \bowtie R_{1}$. Then $\left|R_{1}\right|=\left|R_{2}\right|$.

Proof. This follows from lemmas 54 and 60, since $R_{1}$ and $R_{2}$ are operationally equivalent by definition.

Let now $g_{1}$ and $g_{2}$ be an arbitrary pair of systems in $G$, and $\mathscr{V}$ a homogeneous GUR. By the homogeneity principle 1 , there exists $\mathscr{T}$ such that for every region $R_{1} \ni g_{1}$ there is a region $R_{2} \ni g_{2}$ homologous through $\mathscr{T}$ to $R_{1}$. The set of these transformations is denoted as $T_{\mathscr{V}}$, i.e.

$$
\begin{aligned}
T_{\mathscr{V}}:=\{\mathscr{T} \mid & \exists g_{1}, g_{2} \in G \\
& \left.: \forall R_{1} \ni g_{1} \exists R_{2} \ni g_{2}, R_{2} \bowtie_{\mathscr{T}} R_{1}\right\} .
\end{aligned}
$$

Lemma 62. Let $\mathscr{V}$ satisfy homogeneity, and let $\mathscr{T} \in T_{\mathscr{V}}$. Then there exists a permutation $\pi$ : $G \rightarrow G$ such that if $R_{2} \bowtie_{\mathscr{T}} R_{1}$ it is

$$
R_{2}=\pi\left(R_{1}\right)
$$

Proof. By definition, if $\mathscr{T} \in T_{\mathscr{V}}$ there exist $g_{1}, g_{2} \in G$ such that for every $S_{1} \ni g_{1}$ there is $S_{2} \ni g_{2}$ such that $S_{2} \triangle_{\mathscr{T}} S_{1}$. Let now $R_{2} \bowtie_{\mathscr{T}} R_{1}$, and $f_{1} \in R_{1}$. Consider the finite region $V_{1}:=\left\{f_{1}, g_{1}\right\}$. Then there is a region $V_{2} \ni g_{2}$ such that $V_{2} \bowtie_{\mathscr{T}} V_{1}$, and by lemma 61 , one has $\left|V_{2}\right|=\left|V_{1}\right|=2$. Consequently, it must be $V_{2}=\left\{f_{2}, g_{2}\right\}$. Moreover, since we know that $g_{2} \bowtie_{\mathscr{T}} g_{1}$, by definitions 41,42 , and 43 , it is $f_{2} \bowtie \mathscr{T} f_{1}$. Thus, for every $f_{1} \in R_{1}$ there is a unique $f_{2} \in R_{2}$ such that $f_{2} \bowtie \mathscr{T} f_{1}$. We then define $\pi: G \rightarrow G$ by setting $\pi\left(f_{1}\right):=f_{2}$. By lemma 55 the map $\pi$ is both injective and surjective, and thus it is a permutation of $G$.

In the following, given $\mathscr{T} \in T_{\mathscr{V}}$, we will denote $\mathscr{T}=\mathscr{T}_{\pi}$ where $\pi$ is the permutation of $G$ in lemma 62.

Lemma 63. Let $\left(G, \mathrm{~A}, \mathscr{V}^{\dagger}\right)$ be a homogeneous GUR, and $\pi \in \Pi_{\mathscr{V}}$. Then the GUR $\mathscr{T}_{\pi}$ is unique.

Proof. Let $\mathscr{T}_{\pi}, \mathscr{S}_{\pi} \in T_{\mathscr{V}}$ correspond to the same permutation $\pi$. Let $a \in \llbracket \overline{\mathrm{A}}_{G} \overline{\mathrm{C}} \rrbracket_{Q \mathbb{R}}$. Then by lemma 28 one has $a=\mathscr{A}^{\dagger} e_{G^{\prime}}$, with $\mathscr{A} \in \llbracket \mathrm{A}_{G} \mathrm{C} \rightarrow$ $\mathrm{A}_{G} \mathrm{C} \rrbracket_{Q \mathbb{R}}$. Thus

$$
\begin{aligned}
& \left(\mathscr{S}_{\pi} \mathscr{T}_{\pi}^{-1}\right)^{\dagger} a=\left[\left(\mathscr{S}_{\pi} \mathscr{T}_{\pi}^{-1}\right)^{\dagger} \otimes \mathscr{I}_{\mathrm{C}}^{\dagger}\right] \mathscr{A}^{\dagger} e_{G^{\prime}} \\
& \quad=\left(\mathscr{T}_{\pi}^{-1 \dagger} \mathscr{S}_{\pi}^{\dagger} \otimes \mathscr{I}_{\mathrm{C}}^{\dagger}\right) \mathscr{A}^{\dagger}\left(\mathscr{S}_{\pi}^{-1 \dagger} \mathscr{T}_{\pi}^{\dagger} \otimes \mathscr{I}_{\mathrm{C}}^{\dagger}\right) e_{G^{\prime}}
\end{aligned}
$$


where $G^{\prime}=G \cup h_{0}$ and $\mathrm{A}_{h_{0}} \cong \mathrm{C}$. Now, by definition

$$
\begin{aligned}
\left(\mathscr{S}_{\pi}^{\dagger} \otimes \mathscr{I}_{\mathrm{C}}^{\dagger}\right) & \mathscr{A}^{\dagger}\left(\mathscr{S}_{\pi}^{-1 \dagger} \otimes \mathscr{I}_{\mathrm{C}}^{\dagger}\right) \\
& =\left(\mathscr{T}_{\pi}^{\dagger} \otimes \mathscr{I}_{\mathrm{C}}^{\dagger}\right) \mathscr{A}^{\dagger}\left(\mathscr{T}_{\pi}^{-1 \dagger} \otimes \mathscr{I}_{\mathrm{C}}^{\dagger}\right) \\
& =\left(\mathscr{T}_{i}^{\dagger} \otimes \mathscr{I}_{\mathrm{C}}^{\dagger}\right) \mathscr{A}^{\dagger}\left(\mathscr{T}_{i}^{-1 \dagger} \otimes \mathscr{I}_{\mathrm{C}}^{\dagger}\right)
\end{aligned}
$$

which implies

$$
\left(\mathscr{S}_{\pi} \mathscr{T}_{\pi}^{-1}\right)^{\dagger} a=a .
$$

Since the latter holds for every $\mathrm{C}$ and every $a$, we conclude that $\mathscr{S}_{\pi}=\mathscr{T}_{\pi}$.

Corollary 16. The set $T_{\mathscr{V}}=\left\{\mathscr{T}_{\pi}\right\}$ is a representation of a group $\Pi_{\mathscr{V}}=\left\{\pi \mid \mathscr{T}_{\pi} \in T_{\mathscr{V}}\right\}$ of permutations of $G$.

We can summarise the above results as follows. For every pair $g_{1}, g_{2}$ there exists a reversible map $\pi: G \rightarrow G$ such that $\pi\left(g_{1}\right)=g_{2}$ and a reversible transformation $\mathscr{T}_{\pi}$ of $\llbracket \mathrm{A}_{G} \rrbracket_{Q}$ such that $\mathscr{T}_{\pi}^{-1} \cdot \mathscr{T}_{\pi}$ is an automorphism of $\llbracket \mathrm{A}_{G} \rightarrow \mathrm{A}_{G} \rrbracket_{Q}$ and for every $R \subset G$ and every system $\mathrm{C}$

$$
\begin{aligned}
\llbracket \mathrm{A}_{R}^{(G)} \mathrm{C} \rightarrow & \mathrm{A}_{R}^{(G)} \mathrm{C} \rrbracket_{Q *} \\
& =\mathscr{T}_{\pi}^{-1} \llbracket \mathrm{A}_{\pi(R)}^{(G)} \mathrm{C} \rightarrow \mathrm{A}_{\pi(R)}^{(G)} \mathrm{C} \rrbracket_{Q *} \mathscr{T}_{\pi},
\end{aligned}
$$

and $\mathscr{T}_{\pi}$ leaves the GUR $\mathscr{V}$ invariant, i.e.

$$
\mathscr{T}_{\pi}^{-1} \mathscr{V} \mathscr{T}_{\pi}=\mathscr{V}
$$

The transformations $\pi$ clearly form a group $\Pi_{\mathscr{V}}$ whose action $\Pi_{\mathscr{V}} \times G \rightarrow G$ is transitive.

We now proceed considering the second part of the homogeneity principle, i.e. the statement that discrimination of every pair $g_{1} \neq g_{2}$ is possible relative to some third element $e \in G$. The main consequence is that the action $\Pi_{\mathscr{V}} \times G \rightarrow G$ is free.

Lemma 64. Let $\mathscr{V}$ satisfy the homogeneity principle 1. If $e \neq \pi \in \Pi_{\mathscr{V}}$, there is no element $g$ of $G$ such that $\pi(g)=g$.

Proof. Let $\mathscr{T}_{\pi} \in T_{\mathscr{V}}$. Suppose that there is $g \in G$ such that $\pi(g)=g$. Then for every region $R_{1} \ni g$, the region $R_{2}:=\pi\left(R_{1}\right)$ contains $g$ and one has $R_{2} \bowtie_{\mathscr{T}} R_{1}$, with $g \bowtie_{\mathscr{T}} g$. This is in contradiction with definition 45 , i.e. with the homogeneity principle 1 .

Corollary 17. Let $\Pi_{\mathscr{V}}$ be the group of permutations of $G$ such that $T_{\mathscr{V}}$ is a representation of $\Pi_{\mathscr{V}}$. The action of $\Pi_{\mathscr{V}}$ on $G$ is regular.
We can now summarise all the results that we drew from homogeneity as follows: for every $g_{1}, g_{2} \in G$ there exists a permutation $\pi$ of $G$ such that $\pi\left(g_{1}\right)=g_{2}$, and a GUR $\mathscr{T}_{\pi}$ of $\llbracket \overline{\mathrm{A}}_{G} \rrbracket_{Q \mathbb{R}}$ such that for every $R \in \mathrm{R}^{\left(G^{\prime}\right)}$ and $\mathrm{C}$

$$
\begin{aligned}
\llbracket \mathrm{A}_{R}^{(G)} \mathrm{C} & \rightarrow \mathrm{A}_{R}^{(G)} \mathrm{C} \rrbracket_{Q *} \\
& =\mathscr{T}_{\pi}^{-1} \llbracket \mathrm{A}_{\pi(R)}^{(G)} \mathrm{C} \rightarrow \mathrm{A}_{\pi(R)}^{(G)} \mathrm{C} \rrbracket_{Q *} \mathscr{T}_{\pi},
\end{aligned}
$$

and $\mathscr{T}_{\pi}$ leaves the evolution invariant, i.e.

$$
\mathscr{T}_{\pi}^{-1} \mathscr{V} \mathscr{T}_{\pi}=\mathscr{T}
$$

The transformations $\pi$ form a group $\Pi_{\mathscr{V}}$ that acts regularly (i.e. transitively and freely) on $G$.

Now, it is clear that, chosen $e \in G$, the elements of $G$ can be identified with the elements of $\Pi_{\mathscr{V}}$, as the action of $\Pi_{\mathscr{V}}$ on $G$ is regular. In other therms, $G$ is a principal homogeneous space for $\Pi_{\mathscr{V}}$, and there is a bijection $\Pi_{\mathscr{V}} \leftrightarrow G$. However, the homogeneity principle implies an even stronger result: the set $G$ can be seen as the set of vertices of a special Cayley graph of $\Pi_{\mathscr{V}}$. The following arguments, largely inspired to Refs. [25, 46], make the mentioned result rigorous.

As a consequence of homogeneity, one has

$$
\pi(g) \longmapsto \pi\left(g^{\prime}\right) \Leftrightarrow g \longmapsto g^{\prime} .
$$

In other words, $N_{\pi(g)}^{ \pm}=\pi\left(N_{g}^{ \pm}\right)$for $\pi \in \Pi_{\mathscr{V}}$, as can be checked starting from the definition of $N_{g}^{ \pm}$. The proof is provided in Appendix D.

Let now $g \in G$ and $\pi \in \Pi_{\mathscr{V}}$ such that $\pi(e)=g$. One can then label the elements $g^{\prime} \in N_{g}^{ \pm}$by $\pi^{-1}\left(g^{\prime}\right) \in N_{e}^{ \pm}=\left\{h_{i}^{ \pm 1}\right\}_{i=1}^{k}$. Notice that for the moment we have no reason to claim that $k<\infty$. We will associate all the pairs $\left(g, g^{\prime}\right) \in E$ with the color $h_{i}$ if $g=\pi(e)$ and $g^{\prime}=\pi\left(h_{i}\right)$ for some $\pi \in \Pi_{\mathscr{V}}{ }^{2}$. This construction enriches the graph $\Gamma(G, E)$, which becomes a vertex-transitive coloured directed graph, with colours corresponding to the labels $h_{i} \in N_{e}^{+}$

Given two elements $g, g^{\prime} \in G$, a path $p$ from $g$ to $g^{\prime}$ is an array of elements $p=\left(g_{1}, g_{2}, \ldots, g_{k}\right) \in$

${ }^{2}$ In principle, the colour of an edge $\left(g, g^{\prime}\right)$ might be ambiguously defined if there existed two permutations $\pi, \sigma \in \Pi_{\mathscr{V}}$ such that $\pi^{-1}(g)=\sigma^{-1}(g)=e$ and $\pi^{-1}\left(g^{\prime}\right), \sigma^{-1}\left(g^{\prime}\right) \in N_{e}^{ \pm}$, but $\pi^{-1}\left(g^{\prime}\right) \neq \sigma^{-1}\left(g^{\prime}\right)$. However, due to lemma 64 , this is never the case, since it would imply that $\sigma \pi^{-1}\left(g^{\prime}\right) \neq g^{\prime}$, and thus $\sigma \pi^{-1} \neq e$, but $\left[\sigma \pi^{-1}\right](g)=\sigma(e)=g$. 
$G^{\times k}$ such that, setting $g_{0}:=g$ and $g_{k+1}:=g^{\prime}$, one has

$$
\forall 0 \leq i \leq k \quad\left(g_{i} \longmapsto g_{i+1}\right) \vee\left(g_{i+1} \longmapsto g_{i}\right) .
$$

The lenght of a path $p$, denoted $\ell(p)$ is $k+1$ if $p \in$ $G^{\times k}$. In the following we will restrict attention to the case where $\Gamma(G, E)$ is connected, i.e. for every two elements $g, g^{\prime} \in G$ there exists a path from $g$ to $g^{\prime}$. The set of paths from $g$ to $g^{\prime}$ will be denoted by $\mathrm{p}_{g, g^{\prime}}^{+}$. We can now introduce the graph metric of $\Gamma(G, E)$ as follows. Let $g, g^{\prime} \in G$. Then we define

$$
d_{\Gamma}\left(g, g^{\prime}\right):=\min _{p \in \mathrm{p}_{g, g^{\prime}}^{+}} \ell(p)
$$

We remark that $d_{\Gamma}\left(g, g^{\prime}\right)=d_{\Gamma}\left[\pi(g), \pi\left(g^{\prime}\right)\right]$ for all $\pi \in \Pi_{\mathscr{V}}$, since $g_{1}, \ldots, g_{k}$ is a path from $g$ to $g^{\prime}$ if and only if $\pi\left(g_{1}\right), \ldots, \pi\left(g_{k}\right)$ is a path from $\pi(g)$ to $\pi\left(g^{\prime}\right)$ due to condition (105).

We now use the alphabet $N_{e}^{+} \cup N_{e}^{-}$to form arbitrary words, obtaining a free group F: composition corresponds to word juxtaposition, with the empty word $\lambda$ representing the identity, and the formal rule $h_{i} h_{i}^{-1}=h_{i}^{-1} h_{i}=\lambda$. An element $w=h_{i_{1}}^{p_{1}} h_{i_{2}}^{p_{2}} \ldots h_{i_{n}}^{p_{n}}$ of $F$-with $p_{j} \in\{-1,1\}$ thus corresponds to a path on the graph, where the symbol $h_{i}^{-1} \in N_{e}^{-}$denotes a backwards step along the arrow $h_{i}$. For every $h_{i_{1}}^{p_{1}} h_{i_{2}}^{p_{2}} \ldots h_{i_{m}}^{p_{m}}=$ $w \in F$, one has $w^{-1}=h_{i_{m}}^{-p_{m}} \ldots h_{i_{2}}^{-p_{2}} h_{i_{1}}^{-p_{1}}$.

The action $M: F \times G \rightarrow G$ of words $w=$ $h_{i_{1}}^{p_{1}} \ldots h_{i_{m-1}}^{p_{m-1}} h_{i_{m}}^{p_{m}} \in F$ on elements $g \in G$ can be defined as follows

$$
\begin{aligned}
M(w, g) & =M\left(h_{i_{1}}^{p_{1}} h_{i_{2}}^{p_{2}} \ldots h_{i_{m}}^{p_{m}}, g\right) \\
& :=M\left[h_{i_{2}}^{p_{2}} \ldots h_{i_{m}}^{p_{m}}, M\left(h_{i_{1}}^{p_{1}}, g\right)\right],
\end{aligned}
$$

where the action of $h_{i} \in S$ on the elements $g \in G$ is defined as $M\left(h_{i}, g\right)=g^{\prime}$ if there exists $\pi \in \Pi_{\mathscr{V}}$ such that $\pi^{-1}(g)=e$ and $\pi^{-1}\left(g^{\prime}\right)=h_{i}$, while $M\left(h_{i}^{-1}, g\right)=g^{\prime}$ if there exists $\pi \in \Pi_{\mathscr{V}}$ such that $\pi^{-1}(g)=h_{i}$ and $\pi^{-1}\left(g^{\prime}\right)=e^{3}$. In the following we will use the shortcut

$$
g w:=M(w, g) .
$$

\footnotetext{
${ }^{3}$ The action is well defined. Suppose indeed that there are two elements $f, f^{\prime}$ both satisfying the definition of $M\left(h_{i}, g\right)$. This means that there exist $\pi, \sigma$ such that $\pi^{-1}(g)=\sigma^{-1}(g)=e$, while $\pi^{-1}(f)=\sigma^{-1}\left(f^{\prime}\right)=h_{i}$. However, according to lemma 64 , the first condition implies that $\sigma=\pi$. By the same lemma, the second condition then implies that $f=f^{\prime}$. A similar argument shows that $M\left(h_{i}^{-1}, g\right)$ is well defined.
}

This notation is particularly handy, since

$$
g\left(h_{i_{1}}^{p_{1}} h_{i_{2}}^{p_{2}} \ldots h_{i_{m}}^{p_{m}}\right)=\left(g h_{i_{1}}^{p_{1}}\right) h_{i_{2}}^{p_{2}} \ldots h_{i_{m}}^{p_{m}} .
$$

For every $w \in F$, and for every $g \in G$ and for every permutation $\pi \in \Pi_{\mathscr{V}}$, we now show that $\pi(g w)=\pi(g) w$. The first step consists in proving the result for $w=h_{i}^{ \pm 1}$. Let $g^{\prime}=g h_{i}$, namely by definition $\sigma^{-1}(g)=e$ and $\sigma^{-1}\left(g^{\prime}\right)=h_{i}$ for some (unique) $\sigma \in \pi_{\mathscr{V}}$. By Eq. (105) it is $\pi(g) \longmapsto \pi\left(g^{\prime}\right)$. Moreover, if we define $f:=\pi(g)$ and $f^{\prime}:=\pi\left(g^{\prime}\right)$ we have

$$
\begin{aligned}
& h_{i}=\sigma^{-1}\left(g^{\prime}\right)=\sigma^{-1} \pi^{-1}\left(f^{\prime}\right), \\
& e=\sigma^{-1}(g)=\sigma^{-1} \pi^{-1}(f),
\end{aligned}
$$

namely $f^{\prime}=f h_{i}$. This implies

$$
\pi\left(g h_{i}\right)=\pi\left(g^{\prime}\right)=\pi(g) h_{i} .
$$

Similarly, if $g^{\prime}=g h_{i}^{-1}$ by definition $\sigma^{-1}(g)=h_{i}$ and $\sigma^{-1}\left(g^{\prime}\right)=e$ for some (unique) $\sigma \in \pi_{\mathscr{V}}$. Thus, setting again $f:=\pi(g)$ and $f^{\prime}:=\pi\left(g^{\prime}\right)$, we have

$$
\begin{aligned}
& h_{i}=\sigma^{-1}(g)=\sigma^{-1} \pi^{-1}(f), \\
& e=\sigma^{-1}\left(g^{\prime}\right)=\sigma^{-1} \pi^{-1}\left(f^{\prime}\right),
\end{aligned}
$$

namely $f^{\prime}=f h_{i}^{-1}$, and

$$
\pi\left(g h_{i}^{-1}\right)=\pi\left(g^{\prime}\right)=\pi(g) h_{i}^{-1} .
$$

Notice that the last result implies that for every pair $f, g \in G$, given $\pi \in \Pi_{\mathscr{V}}$ such that $\pi(f)=g$, there is a bijection $N_{f}^{ \pm} \leftrightarrow N_{g}^{ \pm}$, given by

$$
N_{\pi(g)}^{ \pm}=\pi\left(N_{g}^{ \pm}\right)
$$

One can now prove that $\pi(f w)=\pi(f) w$ by induction on the length $l(w)$ of the word $w$. Indeed, we know that it is true for $l(w)=1$. Suppose now that for $l(w)=n-1$ one has $\pi(f w)=\pi(f) w$, and consider $w^{\prime}$ with $l\left(w^{\prime}\right)=n$. Then $w^{\prime}=w h_{i}^{p}$ with $l(w)=n-1$ and $p= \pm 1$. We have

$$
\begin{aligned}
\pi\left(f w^{\prime}\right) & =\pi\left(f w h_{i}^{p}\right)=\pi\left[(f w) h_{i}^{p}\right] \\
& =\pi(f w) h_{i}^{p}=\pi(f) w h_{i}^{p}=\pi(f) w^{\prime},
\end{aligned}
$$

where the induction hypothesis is used in the fourth equality.

Let us now suppose that for some $f \in G$ and some word $w \in F$ one has $f w=f$. Then for every $f^{\prime} \in G$ one can take $\pi$ such that $\pi(f)=f^{\prime}$, thus obtaining

$$
f^{\prime} w=\pi(f) w=\pi(f w)=\pi(f)=f^{\prime} .
$$


Thus, if a path $w \in F$ is closed starting from $f \in G$, then it is closed also starting from any other $g \in G$.

We can now easily see that the subset $R$ of $F$ corresponding to words $r$ such that $g r=g$ for all $g \in G$ is a normal subgroup. Indeed, $R$ is a subgroup because the juxtaposition of two words $s, s^{\prime} \in R$ is again a word $s s^{\prime} \in R$, and for every word $s \in R$ also $s^{-1} \in R$. To prove that $R$ is normal in $F$ we just show that it coincides with its normal closure, i.e. for every $w \in F$ and every $r \in$ $R$, we have $w r w^{-1} \in R$. Indeed, defining for arbitrary $g$ the element $g^{\prime}:=g w$, we have $g^{\prime} w^{-1}=g$, and thus $g w r w^{-1}=g^{\prime} r w^{-1}=g^{\prime} w^{-1}=g$, namely $w r w^{-1} \in R$.

We thus identified a normal subgroup $R$ containing all the words $r$ corresponding to closed paths. If one takes the quotient $F / R$, one obtains a group whose elements are equivalence classes of words in $F$. If we label an arbitrary element of $G$ by $e$, it is clear that the elements of $G$ are in one-to-one correspondence with the vertices of $G$, since for every $g \in G$ there is one and only one class in $F / R$ whose elements lead from $e$ to $g$. We can then write $g=w$ for every $w \in F$ such that $w$ represents a path leading from $e$ to $g$. Notice that the elements of $F / R$, i.e. equivalence classes of words $[w]_{R}$, correspond to elements of $G$, i.e. for every $g \in G$ there is a unique class $[w]_{R}$ such that $g=[w]_{R}$. The criterion for class membership of words is very simple: $w \in g$ iff $w$ connects the vertex $e \in G$ to the vertex $g \in G$.

As a consequence of the above arguments, we can now show that for every $\pi \in \Pi_{\mathscr{V}}$ and for every $g=[w]_{R} \in G$, one has $\pi(g)=[s w]_{R}$ for a fixed word $s \in F$. Indeed, consider $g_{0}=\left[w_{0}\right]_{R}$. Let $\left[w^{\prime}\right]_{R}=\pi\left(g_{0}\right)$. Then $w^{\prime}=w^{\prime} w_{0}^{-1} w_{0}=s w_{0}$, with $s:=w^{\prime} w_{0}^{-1}$, and $\pi\left(g_{0}\right)=\left[s w_{0}\right]_{R}$. Let now $f \in G$. We can always find $t \in F$ so that $f=\left[w_{0} t\right]_{R}$, e.g. by setting $t:=w_{0}^{-1} z, f=[z]_{R}$. Then we have $\pi(f)=\pi\left(\left[w_{0} t\right]_{R}\right)=\pi\left(g_{0} t\right)=\pi\left(g_{0}\right) t=\left[s w_{0} t\right]_{R}=$ $[s z]_{R}$, where we use the definition $\pi(k) r:=[x r]_{R}$ with $\pi(k)=[x]_{R}$. This definition clearly makes sense, since when $[a]_{R}=[b]_{R}$ and $[r]_{R}=[u]_{R}$, also $[a r]_{R}=[b u]_{R}$.

In technical terms, the graph $\Gamma(G, E)=$ $\Gamma(G, S)$ is the Cayley graph of the group $G=$ $F / R$. Homogeneity thus implies that the set $G$ is a group $G$ that can be presented as $G=\langle S \mid R\rangle$, where $S$ is the set of generators of $G$ and $R$ is the group of relators. In the following, if $h_{i}=h_{i}^{-1}$ we will draw an undirected edge to represent $h_{i}$. The presentation can be chosen by arbitrarily dividing $S$ into $S_{+} \subseteq S$ and $S_{-}:=S_{+}^{-1}$ in such a way that $S_{+} \cup S_{-}=S$. The above arbitrariness is inherent the very notion of group presentation and corresponding Cayley graph, and is exploited in the literature, in particular in the definition of isotropy $[25,57]$.

For convenience of the reader we remind the definition of Cayley graph. Given a group $G$ and a set $S_{+}$of generators of the group, the Cayley graph $\Gamma\left(G, S_{+}\right)$is defined as the colored directed graph having vertex set $G$, edge set $\{(g, g h) \mid g \in$ $\left.G, h \in S_{+}\right\}$, and a color assigned to each generator $h \in S_{+}$. Notice that a Cayley graph is regular -i.e. each vertex has the same degree - and vertex-transitive - i.e. all vertices are equivalent, in the sense that the graph automorphism group acts transitively upon its vertices. The Cayley graphs of a group $G$ are in one to one correspondence with its presentations, with $\Gamma\left(G, S_{+}\right)$corresponding to the presentation $\left\langle S_{+} \mid R\right\rangle$.

\section{Locality}

If a GUR were to represent a physical law, we would like that, in order to determine it, it is sufficient to test it in a finite region and for a finite amount of time. This possibility is granted by homogeneity in conjunction with a second principle - locality - to which the present section is devoted. The locality requirement can be phrased in simple words by stipulating that in order to calculate the effects of a physical law, i) it is possible to adopt a reductionist procedure, decomposing an arbitrary system into elementary parts and then calculating its evolution by evolving every part separately, considering only pairwise interactions between parts, and ii) if the reduction is operated, only finite systems will be involved in the calculation for every elementary part.

In the present section we introduce the precise statement of the locality principle, and analyse its main consequences. In order to make this notion independent of homogeneity, we formulate it for general admissible GURs. In particular, this implies that the mathematical statement of locality alone will not allow for determination of a local GUR by observation of a finite region.

Before giving a formal definition, we provide a 
few heuristic steps leading to the final formulation. Let us start considering an admissible GUR $\left(G, \mathrm{~A}, \mathscr{V}^{\dagger}\right)$, with uniformly bounded neighbourhood, namely such that there exists $k<\infty$ so that for every $g \in G$ it is $\left|N_{g}^{ \pm}\right| \leq k$. We remark that, since $\left|N_{g}^{ \pm}\right| \leq k$, it is $\left|P_{g}^{ \pm}\right| \leq k^{2}$. This also implies that the transformations $\mathscr{S}_{g}^{\prime}$ are transformations of finitely many systems.

In the remainder, we will often omit the labels 0,1 on the wires in diagrams, and adopt the convention that the upper half is labelled 0 and the lower one 1 .

Now, reminding Eq. (92), given a $\in$ $\llbracket \overline{\mathrm{A}}_{R}^{(G)} \mathrm{C} \rrbracket_{Q \mathbb{R}}$, one has

$$
\begin{aligned}
{\left[\left(\mathscr{V}^{\dagger} a\right)_{\left(N_{R}^{-}, 1\right) \mathrm{C}}\right] } & =\left[\left\{\left(\mathscr{V}_{R}^{\dagger} \otimes \mathscr{I}_{\mathrm{C}}^{\dagger}\right) a\right\}_{\left(N_{R}^{-}, 1\right) \mathrm{C}}\right] \\
& =\left[\left\{\left(\mathscr{S}_{N_{R}^{\prime}}^{\dagger} \otimes \mathscr{I}_{\mathrm{C}}^{\dagger}\right) a\right\}_{\left(N_{R}^{-}, 1\right) \mathrm{C}}\right]
\end{aligned}
$$

Thus, we have

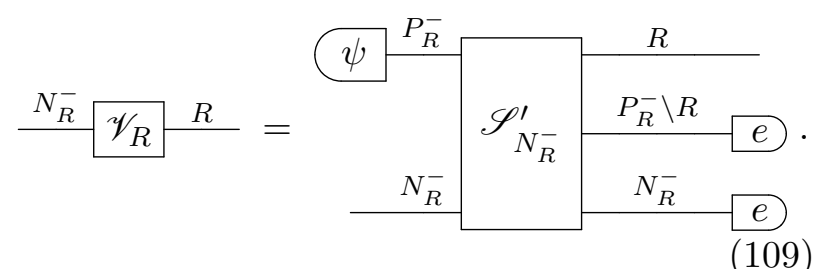

Notice that, by admissibility of the GUR $\mathscr{V}$, and thanks to the no-restriction hypothesis,

$$
\mathscr{V}_{R} \in \llbracket \mathrm{A}_{N_{R}^{-}} \rightarrow \mathrm{A}_{R} \rrbracket 1 .
$$

Moreover, the GUR $\mathscr{V}$ can be equivalently defined starting from its action on $\llbracket \overline{\mathrm{A}}_{G} \rrbracket_{L \mathbb{R}}$, by setting $\mathscr{V}^{\dagger}\left[a_{R}\right]:=\left[\left(\mathscr{V}_{R}^{\dagger} a\right)_{N_{R}^{-}}\right]$. Indeed, following this definition, one can prove that $\mathscr{V}$ is rightinvertible, by the argument discussed in Appendix E. We remark that the construction of the inverse provides a new GUR $\mathscr{W}$.

As a consequence, we have that $\mathscr{W}^{\dagger} \mathscr{V}^{\dagger}=$ $\mathscr{V}^{\dagger} \mathscr{W}^{\dagger}=\mathscr{I}_{G}^{\dagger}$ for some GUR $\mathscr{W}$, and thus $\mathscr{W}=$ $\mathscr{V}^{-1}$. We want to stress here that in proving this result we only use properties (88), (89), (94) and (97) of $\mathscr{S}_{g}^{\prime}$, derived in section 5.4, along with uniform boundedness of the neighbourhoods for the GUR $\mathscr{V}$ (see Appendix E for the details).

Let us now abstract our focus from the situation where a GUR $\left(G, \mathrm{~A}, \mathscr{V}^{\dagger}\right)$ is given. Instead, we will suppose that, given the pair $(G, \mathrm{~A})$, a finite neighbourhood $N_{g}^{+} \subseteq G$ is defined for every $g \in G$, and for $f \in G$ we set

$$
N_{f}^{-}:=\left\{h \in G \mid f \in N_{h}^{+}\right\} .
$$

For $X \in \mathrm{R}^{(G)}$ we then define $N_{X}^{ \pm}:=\bigcup_{g \in X} N_{g}^{ \pm}$, and the sets $P_{g}^{ \pm}$are then defined exactly as for a GUR. Finally, we suppose that a family of maps $\mathscr{S}_{g}^{\prime}$ is defined, with the properties (88) and (89), and an analogue of (94) and (97).

Definition 46 (Local rule). $A \quad k$-local rule $\left(N^{+}, \mathrm{A}, \mathscr{S}^{\prime}\right)$ on $G$ is i) a map $N^{+}: G \rightarrow \mathrm{R}^{(G)}$ that associates $g \in G$ with a finite region $N_{g}^{+} \in$ $\mathrm{R}^{(G)}$ such that $\left|N_{g}^{+}\right|,\left|N_{g}^{-}\right| \leq k$ for every $g \in G$; ii) a map $\mathscr{S}^{\prime}: g \mapsto \mathscr{S}_{g}^{\prime}$, where $\mathscr{S}_{g}^{\prime}$ are maps in $\llbracket \mathrm{A}_{\left(N_{g}^{+}, 0\right)} \mathrm{A}_{(g, 1)} \rightarrow \mathrm{A}_{\left(N_{g}^{+}, 0\right)} \mathrm{A}_{(g, 1)} \rrbracket 1$ with the following properties for every $f, g \in G$ and $R, S \in \mathrm{R}^{(G)}$ :

$$
\begin{aligned}
& \text { 1. } \mathscr{S}_{g}^{\prime} \mathscr{S}_{g}^{\prime}=\mathscr{I}_{\mathrm{A}_{N_{g}^{+}} \mathrm{A}_{g}} \text {; } \\
& \text { 2. } \mathscr{S}_{g}^{\prime} \mathscr{S}_{f}^{\prime}=\mathscr{S}_{f}^{\prime} \mathscr{S}_{g}^{\prime} \text {; } \\
& \text { 3. for every } \mathscr{C} \in \llbracket \mathrm{A}_{(R, 0) \cup(S, 1)} \mathrm{C} \rightarrow \mathrm{A}_{(R, 0) \cup(S, 1)} \mathrm{C} \rrbracket
\end{aligned}
$$

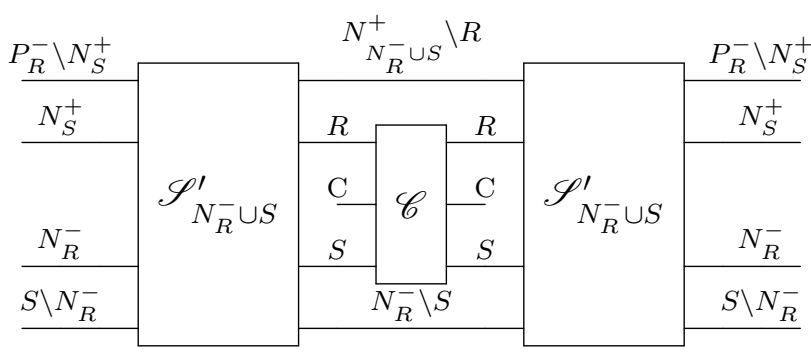

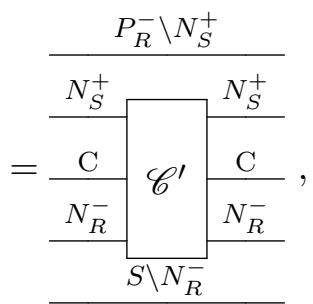

$$
\text { where } \mathscr{S}_{X}^{\prime}:=\prod_{g \in X} \mathscr{S}_{g}^{\prime} \text { for every } X \subseteq G \text {. }
$$

In the following, we will often omit the subscript $X$ in $\mathscr{S}_{X}^{\prime}$ in the diagrams, since $X$ corresponds to the label of the lower half wires. Moreover, in order to make the equations lighter, from now on we will always write or draw transformations and effects that do not involve explicitly the external system $\mathrm{C}$, however it will always be assumed that the conditions hold also locally on extended systems, unless explicitly stated otherwise.

Remark 8. Notice that for a transformation $\mathscr{C}$ of the form $\mathscr{C}_{0} \otimes \mathscr{I}_{\left(R \backslash R^{\prime}, 0\right) \cup\left(S \backslash S^{\prime}, 1\right)}$, due to the definition of $\mathscr{S}_{N_{R}^{-} \cup S}^{\prime}$ and the property 2 of $\mathscr{S}_{g}^{\prime}$, one 
has that
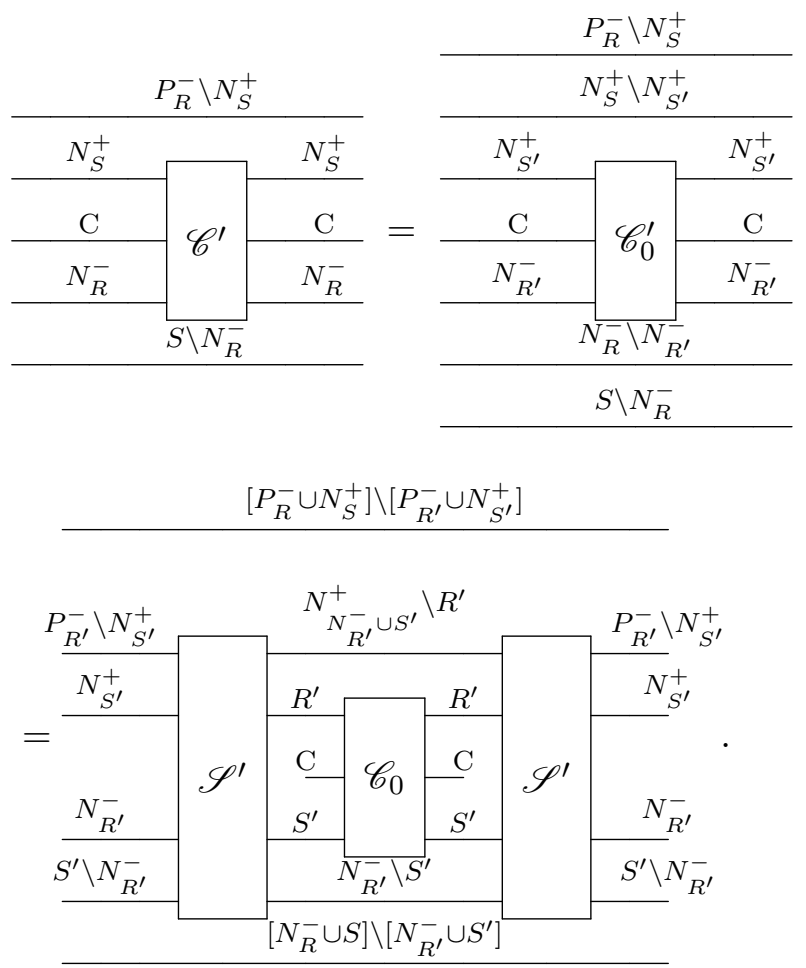

The first property that we prove is the following.

Lemma 65. Given a local rule $\left(N^{+}, \mathrm{A}, \mathscr{S}^{\prime}\right)$ on $G$, the following identities hold:

1. For every $S \in \mathrm{R}^{(G)}$, and every $\mathscr{A} \in \llbracket \mathrm{A}_{S} \mathrm{C} \rightarrow$ $\mathrm{A}_{S} \mathrm{C} \rrbracket$,

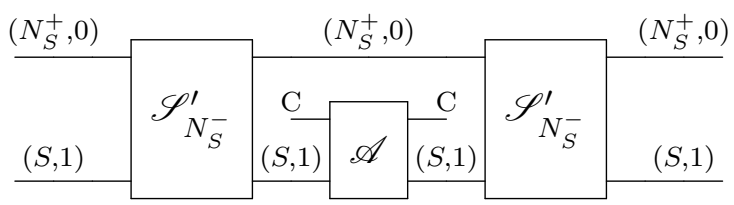

$$
=\frac{\frac{\left(N_{S}^{+}, 0\right)}{\mathrm{C}} \mathscr{A}_{(S, 1)}^{+}}{\mathrm{C}} ;
$$

2. for every $R \in \mathrm{R}^{(G)}$ and every $\mathscr{B} \in \llbracket \mathrm{A}_{R} \mathrm{C} \rightarrow$ $\mathrm{A}_{R} \mathrm{C} \rrbracket$

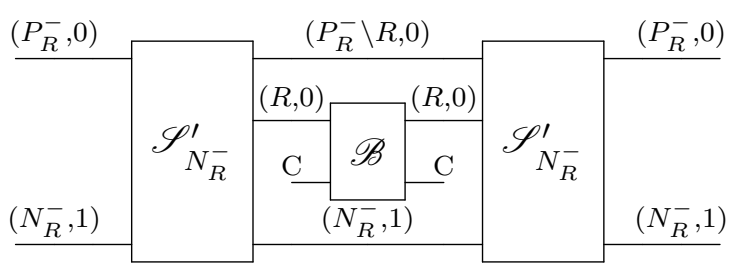

$$
=\frac{\left(P_{R}^{-}, 0\right)}{\stackrel{\mathrm{C}}{\left(N_{R}^{-}, 1\right)} \mathscr{B}^{-}{ }^{\mathrm{C}}\left(N_{R}^{-}, 1\right)} .
$$

Proof. The equalities follow taking $S=\emptyset$ and $R=\emptyset$ in Eq. (110), respectively.

Remark 9. Given a local rule $\left(N^{+}, \mathrm{A}, \mathscr{S}^{\prime}\right)$ we define the forward and backward evolution of local transformations $\mathscr{F} \in \llbracket \mathrm{A}_{R} \mathrm{C} \rightarrow \mathrm{A}_{R} \mathrm{C} \rrbracket$ for a finite region $R \in \mathrm{R}^{(G)}$ through the expressions (111) and (112), as follows

$$
\left[\mathrm{V}_{L}^{ \pm}(\mathscr{F})\right]_{N_{R}^{ \pm} \mathrm{C}}:=\left[\mathscr{F}^{ \pm}\right]_{N_{R}^{ \pm} \mathrm{C}} .
$$

Now, once we established the notion of a local rule and its application to local transformations, we can define the property of a global rule of being reducible to a local rule.

Definition 47 (Reduction to a local rule). We say that the GUR $\left(G, \mathrm{~A}, \mathscr{V}^{\dagger}\right)$ is reducible to a local rule if there exists a $k$-local rule $\left(N^{+}, \mathrm{A}, \mathscr{S}^{\prime}\right)$ for some $k<\infty$ such that for every $R \in \mathrm{R}^{(G)}$ and every $\mathscr{F} \in \llbracket \mathrm{A}_{R} \mathrm{C} \rightarrow \mathrm{A}_{R} \mathrm{C} \rrbracket$

$$
\begin{aligned}
& \left(\mathscr{V} \otimes \mathscr{I}_{\mathrm{C}}\right) \mathscr{F}\left(\mathscr{V}^{-1} \otimes \mathscr{I}_{\mathrm{C}}\right)=\mathrm{V}_{L}^{-}(\mathscr{F}) \otimes \mathscr{I}_{G \backslash N_{R}^{-}}, \\
& \left(\mathscr{V}^{-1} \otimes \mathscr{I}_{\mathrm{C}}\right) \mathscr{F}\left(\mathscr{V} \otimes \mathscr{I}_{\mathrm{C}}\right)=\mathrm{V}_{L}^{+}(\mathscr{F}) \otimes \mathscr{I}_{G \backslash N_{R}^{+}} .
\end{aligned}
$$

We now prove the two main results of this section, which relate local rules and GURs in a oneto-one correspondence.

Theorem 9. Every local rule $\left(N^{+}, \mathrm{A}, \mathscr{S}^{\prime}\right)$ on $G$ identifies a GUR $\left(G^{\prime}, \mathrm{A}, \mathscr{W}^{\dagger}\right)$, with $G^{\prime}=G \times$ $\{0,1\}$, and $\mathscr{W}^{-1}=\mathscr{S}_{G} \mathscr{W} \mathscr{S}_{G}$.

Proof. First of all, one can define the linear maps $\mathscr{W}_{\mathrm{C}}^{\dagger}$ on $\llbracket \overline{\mathrm{A}}_{G} \overline{\mathrm{C}} \rrbracket_{L \mathbb{R}}$ as follows. For $a_{Z \mathrm{C}} \in \llbracket \overline{\mathrm{A}}_{G} \overline{\mathrm{C}} \rrbracket_{L \mathbb{R}}$, with $Z=(R, 0) \cup(S, 1)$, we set

$$
\mathscr{W}^{\dagger}\left[a_{Z \mathrm{C}}\right]:=\left[a_{\left(N_{R}^{-}, 0\right) \cup\left(N_{S}^{+}, 1\right) \mathrm{C}}^{-}\right],
$$

where

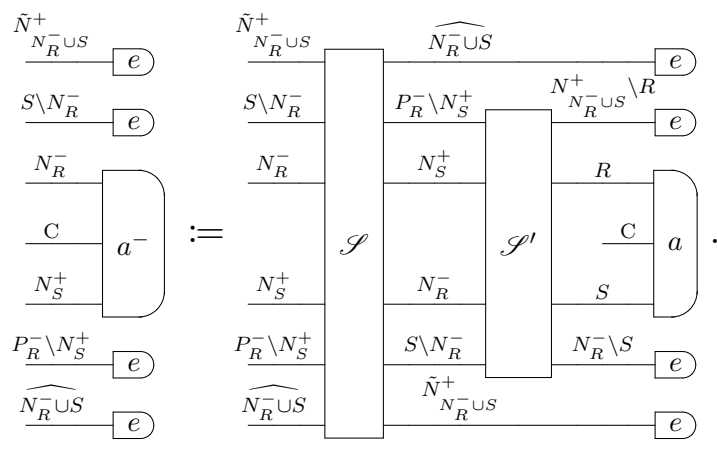

We omitted the subscript for $\mathscr{S}$ and $\mathscr{S}^{\prime}$, as the systems on which the transformations act are 
clear from the context. Finally, $\tilde{N}_{X}^{+}$is a shorthand for $N_{X}^{+} \backslash X$, and $\widehat{X}$ for $X \backslash N_{X}^{+}$. Writing $a=$ $\mathscr{A}^{\dagger} e$, for $\mathscr{A} \in \llbracket \mathrm{A}_{(R, 0) \cup(S, 1)} \mathrm{C} \rightarrow \mathrm{A}_{(R, 0) \cup(S, 1)} \mathrm{C} \rrbracket$, and using Eq. (110), one can easily check that the effect $a^{-}$is actually in $\llbracket \overline{\mathrm{A}}_{\left(N_{R}^{-}, 0\right) \cup\left(N_{S}^{+}, 1\right)} \overline{\mathrm{C}} \rrbracket$. We remark that the map $\mathscr{W}^{\dagger}$ is well defined: for $a \otimes e_{W} \in\left[a_{Z \mathrm{C}}\right]$, with $W=\left(W_{0}, 0\right) \cup\left(W_{1}, 1\right)$, and $W_{0} \cap R=W_{1} \cap S=\emptyset$, one has

$$
\mathscr{W}^{\dagger}\left[\left(a \otimes e_{W}\right)_{\left(R \cup W_{0}, 0\right) \cup\left(S \cup W_{1}, 1\right) \mathrm{C}}\right]=\mathscr{W}^{\dagger}\left[a_{Z \mathrm{C}}\right] .
$$

The above identity is verified exploiting Eq. (110), along with the identity in Remark 8. As to reversibility, for $\left[a_{Z \mathrm{C}}\right]$ with $Z=(R, 0) \cup(S, 1)$, one can define

$$
\mathscr{Z}^{\dagger}\left[a_{Z \mathrm{C}}\right]:=\left[a_{\left(N_{R}^{+}, 0\right) \cup\left(N_{S}^{-}, 1\right) \mathrm{C}}^{+}\right],
$$

where

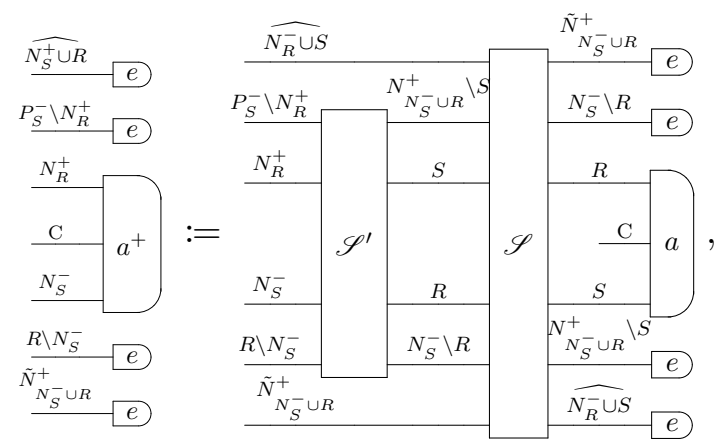

and, similarly to what was done for $\mathscr{W}$, check that $\mathscr{Z}^{\dagger}$ is well defined. One can now verify that $\mathscr{W}^{\dagger} \mathscr{Z}^{\dagger}=\mathscr{Z}^{\dagger} \mathscr{W}^{\dagger}=\mathscr{I}_{\llbracket \bar{A}_{G} \rrbracket_{L \mathbb{R}}}$. The above observations show that $\mathscr{W}^{\dagger}$ and $\mathscr{Z}^{\dagger}=\mathscr{W}^{-1 \dagger}$ act isometrically on $\llbracket \overline{\mathrm{A}}_{G} \rrbracket_{L \mathbb{R}}$, since

$$
\begin{aligned}
\left\|a_{R \mathrm{C}}\right\|_{\mathrm{sup}} & =\left\|\mathscr{Z}^{\dagger} \mathscr{W}^{\dagger} a_{R \mathrm{C}}\right\|_{\mathrm{sup}} \\
& \leq\left\|\mathscr{W}^{\dagger} a_{R \mathrm{C}}\right\|_{\mathrm{sup}} \leq\left\|a_{R \mathrm{C}}\right\|_{\mathrm{sup}} \\
\left\|a_{R \mathrm{C}}\right\|_{\mathrm{sup}} & =\left\|\mathscr{W}^{\dagger} \mathscr{Z}^{\dagger} a_{R \mathrm{C}}\right\|_{\mathrm{sup}} \\
& \leq\left\|\mathscr{Z}^{\dagger} a_{R \mathrm{C}}\right\|_{\mathrm{sup}} \leq\left\|a_{R \mathrm{C}}\right\|_{\text {sup }}
\end{aligned}
$$

Being isometric, both $\mathscr{W}^{\dagger}$ and $\mathscr{W}^{-1 \dagger}$ preserve Cauchy sequences and their equivalence classes. As a consequence, $\mathscr{W}^{\dagger}$ and $\mathscr{W}^{-1 \dagger}$ can be uniquely extended to invertible isometries of $\llbracket \overline{\mathrm{A}}_{G^{\prime}} \rrbracket_{Q \mathbb{R}}$, with $\mathscr{Z}^{\dagger}=\mathscr{W}^{\dagger-1}$. Let now $\rho \in \llbracket \mathrm{A}_{G^{\prime}} \mathrm{C} \rrbracket$, and
$a_{(R, 0) \cup(S, 1) \mathrm{C}} \in \llbracket \overline{\mathrm{A}}_{G^{\prime}} \overline{\mathrm{C}} \rrbracket_{L \mathbb{R}}$. Then

$$
\begin{aligned}
& \left(a_{(R, 0) \cup(S, 1) \mathrm{C}} \mid \hat{\mathscr{W}} \rho\right)=\left(\mathscr{W}^{\dagger} a_{(R, 0) \cup(S, 1) \mathrm{C}} \mid \rho\right) \\
& =\left(\mathscr{S}_{N_{R}^{-} \cup S \cup N_{N_{R}^{-} \cup S}^{+}}^{\dagger} \mathscr{S}_{N_{R}^{-} \cup S}^{\prime \dagger} a_{(S, 0) \cup(T, 1) \mathrm{C}} \mid \rho\right) \\
& =\left(a_{(R, 0) \cup(S, 1) \mathrm{C}} \mid \hat{\mathscr{S}}_{N_{R}^{-} \cup S}^{\prime} \hat{\mathscr{S}}_{N_{R}^{-} \cup S \cup N_{N_{R}^{-} \cup S}^{+}} \rho\right) \\
& =\left(a \mid\left[\hat{\mathscr{S}}_{N_{R}^{-} \cup S}^{\prime} \hat{\mathscr{S}}_{N_{R}^{-} \cup S \cup N_{N_{R}^{-}}^{+} \cup S} \rho\right]_{\mid\left(N_{R}^{-}, 0\right) \cup\left(N_{S}^{+}, 1\right) \mathrm{C}}\right),
\end{aligned}
$$

and clearly this implies that $\hat{\mathscr{W}} \rho_{\mid(R, 0) \cup(S, 1)}$ is a state for every $R, S \in \mathrm{R}^{(G)}$. Then $\hat{\mathscr{W}} \llbracket \mathrm{A}_{G} \mathrm{C} \rrbracket \subseteq$ $\llbracket \mathrm{A}_{G} \mathrm{C} \rrbracket$. The same argument holds for $\hat{\mathscr{W}}^{-1}$, thus $\hat{\mathscr{W}} \llbracket \mathrm{A}_{G} \mathrm{C} \rrbracket=\llbracket \mathrm{A}_{G} \mathrm{C} \rrbracket$. Let now $\mathscr{A}_{T \mathrm{C}} \in$ $\llbracket \mathrm{A}_{G} \mathrm{C} \rightarrow \mathrm{A}_{G} \mathrm{C} \rrbracket_{L}$, with $T=(R, 0) \cup(S, 1)$. One can evaluate $\hat{\mathscr{W}}^{-1} \mathscr{A}_{T} \mathrm{C} \hat{\mathscr{W}}$ by dually evaluating $\mathscr{W}^{\dagger} \mathscr{A}_{T \mathrm{C}}^{\dagger} \mathscr{W}^{-1 \dagger}$. The calculation is a straightforward application of Eq. (110), and shows that $\hat{\mathscr{W}}^{-1} \mathscr{A}_{T \mathrm{C}} \hat{\mathscr{W}} \in \llbracket \mathrm{A}_{G} \mathrm{C} \rightarrow \mathrm{A}_{G} \mathrm{C} \rrbracket_{L}$. The same argument holds for $\hat{\mathscr{W}} \mathscr{A}_{T \mathrm{C}} \hat{\mathscr{W}}^{-1}$, and thus

$$
\hat{\mathscr{W}}^{-1} \llbracket \mathrm{A}_{G} \mathrm{C} \rightarrow \mathrm{A}_{G} \mathrm{C} \rrbracket_{L} \hat{\mathscr{W}}=\llbracket \mathrm{A}_{G} \mathrm{C} \rightarrow \mathrm{A}_{G} \mathrm{C} \rrbracket_{L} .
$$

All the above observations prove that $\mathscr{W}$ is a UR. Finally, being constructed from local transformations, one can easily check that the family $\left\{\mathscr{W}_{\mathrm{C}} \mid\right.$ $\mathrm{C} \in \mathrm{Sys}\}$ is an admissible UR. This concludes the proof that $\left(G^{\prime}, \mathrm{A}, \mathscr{W}^{\dagger}\right)$ is a GUR. Now, comparing the equations defining $a_{\left(N_{R}^{ \pm}, 0\right) \cup\left(N_{S}^{\mp}, 1\right) \mathrm{C}}^{ \pm}$one can easily conclude that $\mathscr{Z}=\mathscr{W}^{-1}=\mathscr{S}_{G} \mathscr{W} \mathscr{S}_{G}$.

Theorem 10. Let $\left(N^{+}, \mathrm{A}, \mathscr{S}^{\prime}\right)$ be a local rule on $G$. Then the GUR $\left(G^{\prime}, \mathrm{A}, \mathscr{W}^{\dagger}\right)$, with $G^{\prime}=G \times$ $\{0,1\}$ in theorem 9, is of the form $\mathscr{W}=\mathscr{V} \otimes \mathscr{V}^{-1}$, where $\left(G, \mathrm{~A}, \mathscr{V}^{\dagger}\right)$ is reducible to $\left(N^{+}, \mathrm{A}, \mathscr{S}^{\prime}\right)$.

The detailed proof can be found in Appendix F. The properties used in the proof are (88), (89), (94), and (97). The first two are required as items 1 and 2 in the definition 46 of a local rule, while the remaining two are a consequence of item 3 as shown by lemma 65 .

In the case of a homogeneous update rule, does the existence of a local rule allow one to determine the full $\left(G, \mathrm{~A}, \mathscr{V}^{\dagger}\right)$ by local tests? One would intuitively expect that the answer is positive, since $\mathscr{S}_{g}^{\prime}=\mathscr{S}_{e}^{\prime}$ are the same for every $g \in G$, by virtue of homogeneity, and thus, knowing $\mathscr{S}_{e}^{\prime}$, one could calculate the action of $\mathscr{V}$ through Eq. (112). On the other hand, after giving the question another thought, one can realise that calculating the action of $\mathscr{V}$ through the maps $\mathrm{V}_{L}^{ \pm}$requires another 
piece of information besides knowing $\mathscr{S}_{e}^{\prime}$. Actually, determining the cellular automaton with local tests also requires knowledge of the regions $N_{R}^{+}$for every $R \in \mathrm{R}^{(G)}$-or, in other words, knowledge of the structure of the graph of influence relations. This information is indeed necessary in order to know how to correctly calculate the transformation $\mathrm{V}_{L}^{ \pm}\left(\mathscr{A}_{R}\right)$ for a given region $R$. Indeed, it might happen that knowledge of finitely many closed paths from the elements $g \in R$ is not sufficient to reconstruct all the new closed paths that appear as the size of the region $R$ increases. This means that one needs infinitely many rules to identify systems in $N_{g_{1}}^{ \pm} \cap N_{g_{2}}^{ \pm}$. However, this is not the case if closed paths in the graph of influence relations can be decomposed into elementary closed paths having a uniformly bounded size, let us say by a constant length $l$. In this case, knowledge of local rules and of the local structure of the graph up to some finite distance $l$ is sufficient to calculate the action of $\mathscr{V}$ in an arbitrary finite region. This is the reason for the following definition.

Definition 48 (Decomposability into bounded regions). We say that a global update rule $\left(G, \mathrm{~A}, \mathscr{V}^{\dagger}\right)$ is decomposable into bounded regions if the closed paths of the influence graph of $\mathscr{V}$ can be decomposed into elementary closed paths of uniformly bounded length.

We are finally in position to formulate the locality principle, which can be thought of as the requirement that finite, local information is needed in order to reconstruct the update rule.

Principle 2 (Locality). The GUR $\left(G, \mathrm{~A}, \mathscr{V}^{\dagger}\right)$ is reducible to a local rule and decomposable into bounded regions.

\section{Cellular Automata}

The principles of homogeneity and locality single out the special class of global rules that we will call cellular automata.

Definition 49. Let $\left(G, \mathrm{~A}, \mathscr{V}^{\dagger}\right)$ be a global update rule obeying the principles of homogeneity and locality. We say that $\left(G, \mathrm{~A}, \mathscr{V}^{\dagger}\right)$ is a cellular automaton.

Notice that the influence graph of a cellular automaton is the Cayley graph of a finitely presented group, i.e. a group that is finitely generated and can be presented through finitely many relators. This fact has the very important consequence that every cellular automaton defines a Cayley graph that is quasi-isometric to some smooth Riemannian manifold of dimension $d \leq 4$ (see [47], sec. IV pag. 90).

An important remark is in order. Thanks to homogeneity, one can easily show that the local rule $\mathscr{S}_{g}^{\prime}$ for a cellular automaton on the Cayley graph $\Gamma\left(G, S_{+}\right)$has the property that $\mathscr{S}_{g}^{\prime}=\mathscr{S}_{e_{G}}^{\prime}$ for every $g \in G$. Thus, cellular automata can be identified by their Cayley graph $\Gamma\left(G, S_{+}\right)$and by a reversible transformation $\mathscr{S}_{e_{G}}^{\prime} \in \llbracket \mathrm{A}_{N_{e_{G}^{+}}^{+}} \mathrm{A}_{e_{G}} \rightarrow$ $\mathrm{A}_{N_{e_{G}^{+}}^{+}} \mathrm{A}_{e_{G}} \rrbracket_{1}$.

\subsection{Results}

We now prove two important theorems regarding cellular automata. The first one shows that, in order to obey the properties of homogeneity and locality, a cellular automaton cannot be in the quasi-local algebra.

Theorem 11. Let $|G|=\infty$. The homogeneous and local cellular automaton $\mathscr{V}$ is an element of $\llbracket \mathrm{A}_{G} \rightarrow \mathrm{A}_{G} \rrbracket_{Q 1}$ if and only if it is trivial: $\mathscr{V}=$ $\mathscr{I}_{G}$.

Proof. Let $\mathscr{V}$ be in the quasi-local algebra, and $\mathscr{V}_{n R_{n}}$ be a sequence of local transformations in the class of $\mathscr{V}$. Then for $\varepsilon>0$ there exists $n_{0}$ such that for every $n \geq n_{0}$ one has

$$
\left\|\mathscr{V}_{n R_{n}}-\mathscr{V}\right\|_{\text {sup }} \leq \varepsilon
$$

Moreover, for $\pi \in \Pi_{\mathscr{V}}$, one has $\mathscr{T}_{\pi} \mathscr{V}_{\pi}^{-1}=\mathscr{V}$. Then

$$
\left\|\mathscr{V}_{n R_{n}}-\mathscr{T}_{\pi} \mathscr{V} \mathscr{T}_{\pi}^{-1}\right\|_{\text {sup }} \leq \varepsilon .
$$

Since $\mathscr{T}_{\pi}$ is a GUR, it is isometric and thus

$$
\begin{aligned}
& \left\|\mathscr{T}_{\pi}^{-1} \mathscr{V}_{n R_{n}} \mathscr{T}_{\pi}-\mathscr{V}\right\|_{\text {sup }} \\
& \quad=\left\|\mathscr{V}_{n R_{n}}-\mathscr{T}_{\pi} \mathscr{V}_{\pi}^{-1}\right\|_{\text {sup }} \leq \varepsilon .
\end{aligned}
$$

Now, this implies that

$$
\left\|\mathscr{V}_{n R_{n}}-\mathscr{T}_{\pi}^{-1} \mathscr{V}_{n R_{n}} \mathscr{T}_{\pi}\right\|_{\text {sup }} \leq 2 \varepsilon .
$$

It is easy to prove that, if $|G|=\infty$, for every $R \in \mathrm{R}^{(G)}$ there must exist $\pi \in \Pi_{\mathscr{V}}$ so that $R \cap$ $\pi(R)=\emptyset$. Suitably choosing $\pi$, condition (115) then takes the form

$$
\begin{aligned}
& \|\mathscr{W} \otimes \mathscr{I}-\mathscr{I} \otimes \mathscr{W}\|_{\text {sup }} \\
& =\|\mathscr{W} \otimes \mathscr{I}-\mathscr{W} \otimes \mathscr{W}+\mathscr{W} \otimes \mathscr{W}-\mathscr{I} \otimes \mathscr{W}\|_{\text {sup }} \\
& \leq 2\|\mathscr{W}-\mathscr{I}\|_{\text {sup }} \leq \varepsilon
\end{aligned}
$$


Being $\varepsilon$ arbitrary, we must conclude that $\mathscr{W}=$ $\mathscr{I}$, namely $\mathscr{V}_{n R_{n}}=1_{\emptyset}$.

The second result pertains the following types of theory.

1. Theories with local discriminability $[8,9$, $15,58]$, or more generally every theory where the algebra of transformations $\mathscr{A} \in$ $\llbracket \mathrm{A}_{1} \ldots \mathrm{A}_{n} \rightarrow \mathrm{A}_{1} \ldots \mathrm{A}_{n} \rrbracket$ is generated by local transformations, i.e. transformations of the form

$$
\mathscr{A}_{i} \in \mathscr{I}_{\overline{\mathrm{A}}_{i}} \otimes \llbracket \mathrm{A}_{i} \rightarrow \mathrm{A}_{i} \rrbracket
$$

where $\overline{\mathrm{A}}_{i}$ is the composite system made of all $\mathrm{A}_{1} \ldots \mathrm{A}_{n}$ except from $\mathrm{A}_{i}$. This class includes, among others, classical information theory and quantum theory.

2. Theories where the algebra of transformations $\mathscr{A} \in \llbracket \mathrm{A}_{1} \ldots \mathrm{A}_{n} \rightarrow \mathrm{A}_{1} \ldots \mathrm{A}_{n} \rrbracket$ is generated by bipartite transformations, i.e. transformations of the form

$$
\mathscr{A}_{i, j} \in \mathscr{I}_{\overline{\mathrm{A}}_{i} \overline{\mathrm{A}}_{j}} \otimes \llbracket \mathrm{A}_{i} \mathrm{~A}_{j} \rightarrow \mathrm{A}_{i} \mathrm{~A}_{j} \rrbracket,
$$

where $\overline{\mathrm{A}}_{i} \overline{\mathrm{A}}_{j}$ is the composite system made of all $A_{1} \ldots A_{n}$ except from $A_{i}$ and $A_{j}$. This case includes the Fermionic theory as well as real quantum theory.

In the above cases, for groups $G$ with suitable properties that we will immediately specify, the admissible local rules for a cellular automaton can be sought considering finite-dimensional systems. In the remainder we will then restrict to the families of theories introduced above.

Before proving this result, we need some preliminary definition and lemma.

Definition 50 (Quotient group). Let $G, H$ be two groups, and suppose that there exists a group homomorphism $\varphi: G \rightarrow H$. Then $H$ is a quotient of $G$. If $H$ is finite, it is called a finite quotient of $G$.

The reason why we are interested in finite quotients is that, under suitable assumptions that will be made rigorous shortly, the same local rule defines an automaton on $G$ and an automaton on any of its finite quotients $H$. This result makes it much easier to provide unitarity conditions in all those cases where the finite quotients of the group $G$ satisfy the mentioned requirements. We now explain the hypotheses in detail, and finally prove the main theorem.

We start remarking that if the group $G$ has a finite presentation

$$
G=\left\langle a_{1}, \ldots, a_{n} \mid r_{1}, \ldots, r_{k}\right\rangle
$$

it is straightforward to verify that any quotient $H$ of $G$ has a finite presentation of the form

$H=\left\langle\varphi\left(a_{1}\right), \ldots, \varphi\left(a_{n}\right) \mid \varphi\left(r_{1}\right), \ldots, \varphi\left(r_{k}\right), b_{1}, \ldots, b_{j}\right\rangle$,

where the new relations $b_{k}$ do not belong to the group $R$, conjugate closure of $\left\{\varphi\left(r_{1}\right), \ldots, \varphi\left(r_{k}\right)\right\}$. When $H$ is a quotient of $G$, referring to the presentation above, we will denote by $R^{\prime}$ the conjugate closure of the group generated by $\left\{\phi\left(r_{l}\right)\right\}_{l=1}^{k} \cup\left\{b_{m}\right\}_{m=1}^{j}$. Moreover, the neighbourhoods of $f$ in $\Gamma\left(H, \phi\left(S_{+}\right)\right)$will be denoted by $N_{f}^{\prime \pm}$ for $f \in H$. We now state two lemmas that we use in the following. The proofs are provided in Appendix $\mathrm{G}$

Lemma 66. Let $N^{+}$be a neighbourhood system corresponding to the Cayley graph $\Gamma\left(G, S_{+}\right)$of a a finitely generated group $G$, and let $H=\phi(G)$ be a finite quotient of $G$ such that for every $h_{a}, h_{b}, h_{c}, h_{d} \in S_{+}$one has $\phi\left(h_{a} h_{b}^{-1}\right) \in R^{\prime}$, if and only if $h_{a} h_{b}^{-1} \in R$ and $\phi\left(h_{a} h_{b}^{-1} h_{c} h_{d}^{-1}\right) \in R^{\prime}$ if and only if $h_{a} h_{b}^{-1} h_{c} h_{d}^{-1} \in R$.

1. $N_{\phi(g)}^{\prime \pm}=\phi\left(N_{g}^{ \pm}\right)$, and $\left|N_{\phi(g)}^{\prime \pm}\right|=\left|N_{g}^{ \pm}\right|$.

2. $P_{\phi(g)}^{\prime \pm}=\phi\left(P_{g}^{ \pm}\right)$, and $\left|P_{\phi(g)}^{\prime \pm}\right|=\left|P_{g}^{ \pm}\right|$.

3. Given $f_{1}, f_{2} \in H$, one can choose $g_{i} \in$ $\phi^{-1}\left(f_{i}\right)$ such that $N_{f_{1}}^{\prime \pm} \cap N_{f_{2}}^{\prime \pm}=\phi\left(N_{g_{1}}^{ \pm} \cap N_{g_{2}}^{ \pm}\right)$ and $\left|N_{f_{1}}^{\prime \pm} \cap N_{f_{2}}^{\prime \pm}\right|=\left|N_{g_{1}}^{ \pm} \cap N_{g_{2}}^{ \pm}\right|$.

4. Given $f_{1}, f_{2} \in H$, one can choose $g_{i} \in$ $\phi^{-1}\left(f_{i}\right)$ such that $P_{f_{2}}^{\prime \mp} \cap N_{f_{1}}^{\prime \pm}=\phi\left(P_{g_{2}}^{\mp} \cap N_{g_{1}}^{ \pm}\right)$ and $\left|P_{f_{2}}^{\prime \mp} \cap N_{f_{1}}^{\prime \pm}\right|=\left|\phi\left(P_{g_{2}}^{\mp} \cap N_{g_{1}}^{ \pm}\right)\right|$.

5. If $g_{2} \notin N_{g_{1}}^{ \pm}$and $\phi\left(g_{2}\right) \in N_{\phi\left(g_{1}\right)}^{\prime \pm}$, it is $P_{g_{1}}^{ \pm} \cap$ $N_{g_{2}}^{\mp}=\emptyset$.

Lemma 67. In addition to the hypotheses of lemma 66, let $\phi\left(h_{a} h_{b}^{-1} h_{c} h_{d}^{-1} h_{e} h_{f}^{-1}\right) \in$ $R^{\prime} \quad$ iff $h_{a} h_{b}^{-1} h_{c} h_{d}^{-1} h_{e} h_{f}^{-1} \in R$ for every $h_{a}, h_{b}, h_{c}, h_{d}, h_{e}, h_{f} \in S_{+}$. Then

1. Given $f_{1}, f_{2} \in H$ with $f_{2} \in P_{f_{1}}^{\prime \pm}$, one has $P_{f_{1}}^{\prime \pm} \cap P_{f_{2}}^{\prime \pm}=\phi\left(P_{g_{1}}^{ \pm} \cap P_{g_{2}}^{ \pm}\right)$, for some $g_{i} \in$ $\phi^{-1}\left(f_{i}\right)$, with $g_{2} \in P_{g_{1}}^{ \pm}$, and $\left|P_{f_{1}}^{\prime \pm} \cap P_{f_{2}}^{\prime \pm}\right|=$ $\left|P_{g_{1}}^{ \pm} \cap P_{g_{2}}^{ \pm}\right|$. 
2. Let $g_{2} \notin P_{g_{1}}^{ \pm}$and $\phi\left(g_{2}\right) \in P_{\phi\left(g_{1}\right)}^{\prime \pm}$. Then $N_{g_{1}}^{ \pm} \cap$ $N_{g_{2}}^{ \pm}=P_{g_{1}}^{ \pm} \cap P_{g_{2}}^{ \pm}=\emptyset$.

Corollary 18. Under the hypotheses of lemma 66, the homomorphism $\phi$ is invertible on $N_{g}^{ \pm}$and $P_{g}^{ \pm}$for every $g \in G$.

In order to make the hypotheses of lemmas 66 and 67 clearer, in Fig. 2 we show two examples of finite quotients of the group $\mathbb{Z}^{2}$, the first one satisfying the hypotheses of lemma 66 but not those of lemma 67 , and the second one satisfying both.

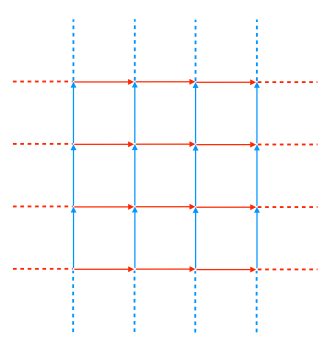

(a)
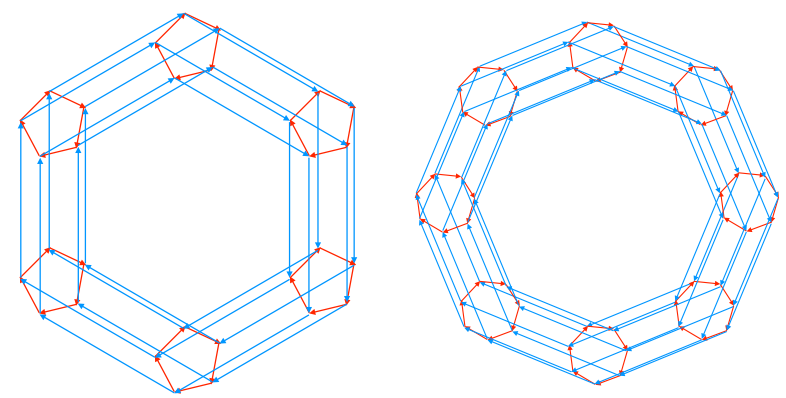

(b)

(c)

Figure 2: (a) The Cayley graph of $\mathbb{Z}^{2}$ presented as $\left\langle a, b \mid a b a^{-1} b^{-1}\right\rangle$; (b) the Cayley graph of the finite quotient of $\mathbb{Z}^{2}\left\langle\phi(a), \phi(b) \mid \phi\left(a b a^{-1} b^{-1}\right), \phi(a)^{6}, \phi(b)^{5}\right\rangle$, satisfying the hypotheses of lemma 66 but not those of 67; (c) the Cayley graph of the finite quotient of $\mathbb{Z}^{2}\left\langle\phi(a), \phi(b) \mid \phi\left(a b a^{-1} b^{-1}\right), \phi(a)^{8}, \phi(b)^{7}\right\rangle$, satisfying the hypotheses of both lemmas 66 and 67 . Notice that we implicitly assume that $S_{+}$contains also $a^{-1}$ and $b^{-1}$, but we do not express the presentation accordingly, to keep the formulas readable. In principle there should be two extra generators $c, d$ (and $\phi(c), \phi(d)$ in the finite quotients), along with the relations $a c$ and $b d$ (and $\phi(a c), \phi(b d)$, accordingly).

We can now prove the following results, which are the core of the final theorem at the end of the section.

Lemma 68. For theories of type 1, under the hypotheses of lemma 66, given a homogeneous local rule $\left(N^{+}, \mathrm{A}, \mathscr{S}^{\prime}\right)$ with neighbourhood system $N^{+}$such that $\left|N_{e_{G}^{+}}^{+}\right|=\left|S_{+}\right|$, the local rule $\left(N^{\prime+}, \mathrm{A}, \tilde{\mathscr{S}}^{\prime}\right)$, with $\tilde{\mathscr{S}}_{e_{H}}^{\prime}:=\mathscr{S}_{e_{G}}^{\prime}$ is welldefined on the Cayley graph $\Gamma\left(H, \phi\left(S_{+}\right)\right)$, with $N_{\phi(g)}^{\prime+}=\phi\left(N_{g}^{+}\right)$. Viceversa, given a local rule $\left(N^{\prime+}, \mathrm{A}, \tilde{\mathscr{S}}^{\prime}\right)$ whose neighbourhood system $N^{\prime+}$ corresponds to a Cayley graph $\Gamma\left(H, \phi\left(S_{+}\right)\right)$, the local rule $\left(N^{+}, \mathrm{A}, \mathscr{S}^{\prime}\right)$, with $\mathscr{S}_{e}^{\prime}:=\tilde{\mathscr{S}}_{e}^{\prime}$ is well-defined on the Cayley graph $\Gamma\left(G, S_{+}\right)$, with $\phi\left(N_{g}^{+}\right)=N_{\phi(g)}^{\prime+}$

Proof. We remark that, by definition of the maps $\mathscr{S}_{g}^{\prime}$ (see Definition 46) a given choice of $\mathscr{S}_{e_{H}}^{\prime}$ is in principle compatible with every Cayley graph with $\left|N_{e_{H}}^{ \pm}\right|=\left|N_{e_{G}}^{ \pm}\right|$, i.e. the same number of generators (we remind that $\mathscr{S}_{g}^{\prime}=\mathscr{S}_{e_{H}}^{\prime} \in$ $\left.\llbracket \mathrm{A}_{N_{e_{G}}^{+}} \mathrm{A}_{e_{G}} \rightarrow \mathrm{A}_{N_{e_{G}^{+}}^{+}} \mathrm{A}_{e_{G}} \rrbracket_{1}\right)$. By lemma 66, the cardinality of $N_{e_{H}}^{\prime+}$ is the same as that of $N_{e_{G}}^{+}$. Then $\tilde{\mathscr{S}}_{e_{H}}^{\prime}=\mathscr{S}_{e_{G}}^{\prime}$ is well defined on $\Gamma\left(H, \phi\left(S_{+}\right)\right)$ iff it is well defined on $\Gamma\left(G, S_{+}\right)$. What remains to be proved is that the map $\tilde{\mathscr{S}}_{e_{H}}^{\prime}=\mathscr{S}_{e_{G}}^{\prime}$ identifies a local rule on the system of neighbourhoods $N^{\prime+}$-namely that Eq. (110) as well as items 1 and 2 in definition 46 hold - iff it does on the system of neighbourhoods $N^{+}$. Item 1 is satisfied by $\tilde{\mathscr{S}}^{\prime}=\mathscr{S}^{\prime}$ by definition in both cases. As to item 2, it is trivial to verify that the only important feature in order to decide whether it holds in any neighbourhood system $N_{e_{H}}^{\prime+}$ is the cardinality of $N_{e_{H}}^{\prime+} \cap N_{f}^{\prime+}$ (or $N_{e_{G}}^{+} \cap N_{g}^{+}$) for every $f \in P_{e_{H}}^{\prime+}$ (or $g \in P_{e_{G}}^{-}$), since this identifies the systems where both $\tilde{\mathscr{S}}_{e_{H}}^{\prime}=\mathscr{S}_{e_{G}}^{\prime}$ and $\tilde{\mathscr{S}}_{f}^{\prime}=\mathscr{S}_{g}^{\prime}$ act non-trivially, namely those where the products $\tilde{\mathscr{S}}_{e_{H}}^{\prime} \tilde{\mathscr{S}}_{f}^{\prime}$ or $\mathscr{S}_{e_{G}}^{\prime} \mathscr{S}_{g}^{\prime}$ might not be commutative. Lemma 66 thus ensures that item 2 is satisfied by $\tilde{\mathscr{S}}^{\prime}$ iff it is by $\mathscr{S}$. Let us then focus on Eq. (110). For theories of type 1, it is sufficient to verify that Eq. (110) holds for $|R|=1$ and $|S|=0$ or $|R|=0$ and $|S|=1$. Both conditions easily follow if we consider lemma 66 .

Lemma 69. For theories of type 2, under the hypotheses of lemma 67, given a homogeneous local rule $\left(N^{+}, \mathrm{A}, \mathscr{S}^{\prime}\right)$ with neighbourhood system $N^{+}$such that $\left|N_{e_{G}}^{+}\right|=\left|S_{+}\right|$, the local rule $\left(N^{\prime+}, \mathrm{A}, \tilde{\mathscr{S}}^{\prime}\right)$, with $\tilde{\mathscr{S}}_{e_{H}}^{\prime}:=\mathscr{S}_{e_{G}}^{\prime}$ is welldefined on the Cayley graph $\Gamma\left(H, \phi\left(S_{+}\right)\right)$, with $N_{\phi(g)}^{\prime+}=\phi\left(N_{g}^{+}\right)$. Viceversa, given a local rule $\left(N^{\prime+}, \mathrm{A}, \tilde{\mathscr{S}}^{\prime}\right)$ whose neighbourhood system $N^{\prime+}$ corresponds to a Cayley graph $\Gamma\left(H, \phi\left(S_{+}\right)\right)$, the local rule $\left(N^{+}, \mathrm{A}, \mathscr{S}^{\prime}\right)$, with $\mathscr{S}_{e}^{\prime}:=\tilde{\mathscr{S}}_{e}^{\prime}$ is 
well-defined on the Cayley graph $\Gamma\left(G, S_{+}\right)$, with $\phi\left(N_{g}^{+}\right)=N_{\phi(g)}^{\prime+}$

Proof. The first part of the proof proceeds exactly as that of lemma 68 . The non trivial part of the proof regards the equivalence of Eq. (110) for both $\mathscr{S}^{\prime}$ on $G$ and $\tilde{\mathscr{S}}^{\prime}$ on $H$. Suppose that $\mathscr{S}^{\prime}$ defines a local rule for the neighbourhood system $N_{g}^{+}$. In theories of type 2, it is sufficient to verify that Eq. (110) holds for regions with $|R|=2,|S|=0$, or $|R|=1,|S|=1$, or $|R|=0$, $|S|=2$. Let then $R, S \subseteq \Gamma\left(H, \phi\left(S_{+}\right)\right)$, with $R=\phi(\tilde{R})$ and $S=\phi(\tilde{S})$. In the first case we have $R=\left\{f_{1}, f_{2}\right\}$, with $\tilde{R}=\left\{g_{1}, g_{2}\right\}$ and $f_{i}=\phi\left(g_{i}\right)$, while $S=\emptyset$. The relevant informations in order to verify Eq. (110) are the cardinalities i) $\left|N_{f_{1}}^{\prime-} \cap N_{f_{2}}^{\prime-}\right|$ and ii) $\left|P_{f_{1}}^{\prime-} \cap P_{f_{2}}^{\prime-}\right|$. If $f_{2} \in P_{f_{1}}^{\prime-}$, by lemma 67 we know that we can choose $g_{1}$ and $g_{2}$ so that $\left|N_{f_{1}}^{\prime-} \cap N_{f_{2}}^{\prime-}\right|=\left|N_{g_{1}}^{-} \cap N_{g_{2}}^{-}\right|$and $\left|P_{f_{1}}^{\prime-} \cap P_{f_{2}}^{\prime-}\right|=\left|P_{g_{1}}^{-} \cap P_{g_{2}}^{-}\right|$. Thus, since Eq. (110) holds for $\mathscr{C} \in \llbracket \mathrm{A}_{\tilde{R}}^{(G)} \rightarrow \mathrm{A}_{\tilde{R}}^{(G)} \rrbracket$, and

$$
\mathrm{A}_{\tilde{R}}^{(G)} \cong \mathrm{A}_{R}^{(H)}, \quad \tilde{\mathscr{S}}^{\prime}=\mathscr{S}^{\prime}
$$

we conclude that Eq. (110) holds also for $\mathscr{C} \in$ $\llbracket \mathrm{A}_{R}^{(H)} \rightarrow \mathrm{A}_{R}^{(H)} \rrbracket$. On the other hand, if $f_{2} \notin P_{f_{1}}^{\prime-}$, we can calculate $\tilde{\mathscr{S}}_{N_{f_{1}, f_{2}}^{\prime-}}^{\prime} \mathscr{C} \tilde{\mathscr{S}}_{N_{f_{1}, f_{2}}^{\prime-}}^{\prime-}$ in two steps. First we apply $\tilde{\mathscr{S}}_{N_{f_{1}}^{\prime-}}^{\prime}=\mathscr{S}_{N_{g_{1}}^{-}}^{\prime}$, treating the system $f_{2}$ as an external system. Reminding that $f_{2} \notin$ $P_{f_{1}}^{\prime-}$, it is $N_{f_{1}}^{\prime-} \cap N_{f_{2}}^{\prime-}=\emptyset$. By Eq. (110), that holds for $\mathscr{S}_{N_{g_{1}}^{-}}^{\prime}$ we then have

\begin{tabular}{|c|c|c|c|c|}
\hline \multicolumn{5}{|c|}{$\left(P_{f_{2}}^{\prime-} \backslash\left[P_{f_{1}}^{\prime-} \cup f_{2}\right], 0\right)$} \\
\hline \multicolumn{3}{|c|}{$\left(f_{2}, 0\right)$} & \multicolumn{2}{|l|}{$\left(f_{2}, 0\right)$} \\
\hline$\left(\overline{\left.P_{f_{1}}^{\prime-}, 0\right)}\right.$ & & $\begin{array}{lll}\left.f_{1}, 0\right) & \mathscr{C} & \left(f_{1}, 0\right) \\
\end{array}$ & \multirow{3}{*}{$\tilde{\mathscr{S}}_{N_{f_{1}}^{-}}^{\prime}$} & $\left(P_{f_{1}}^{\prime-}, 0\right)$ \\
\hline \multirow[b]{2}{*}{$\left(N_{f_{1}}^{\prime-}, 1\right)$} & \multirow[t]{2}{*}{$\tilde{\mathscr{S}}_{N_{f_{1}}^{\prime-}}^{\prime}$} & $\left(P_{f_{1}}^{\prime-} \backslash f_{1}, 0\right)$ & & \multirow{3}{*}{$\left(N_{f_{1}}^{\prime-}, 1\right)$} \\
\hline & & $\left(N_{f_{1}}^{-}, 1\right)$ & & \\
\hline & & $\left(N_{f_{2}}^{\prime-}, 1\right)$ & & \\
\hline
\end{tabular}

$$
\begin{aligned}
& =\frac{\left(P_{f_{1}}^{\prime-} \cup P_{f_{2}}^{\prime-} \backslash f_{2}, 0\right)}{\stackrel{\left(f_{2}, 0\right)}{\left(N_{f_{1}}^{\prime-}, 1\right)} \mathscr{C}^{\prime} \frac{\left(f_{2}^{\prime-}, 0\right)}{\left(N_{f_{1}}^{\prime-}, 1\right)}} . \\
& \left(N_{f_{2}}^{\prime-}, 1\right)
\end{aligned}
$$

Then, we apply $\tilde{\mathscr{S}}_{N_{f_{2}}^{\prime-}}^{\prime}$, obtaining

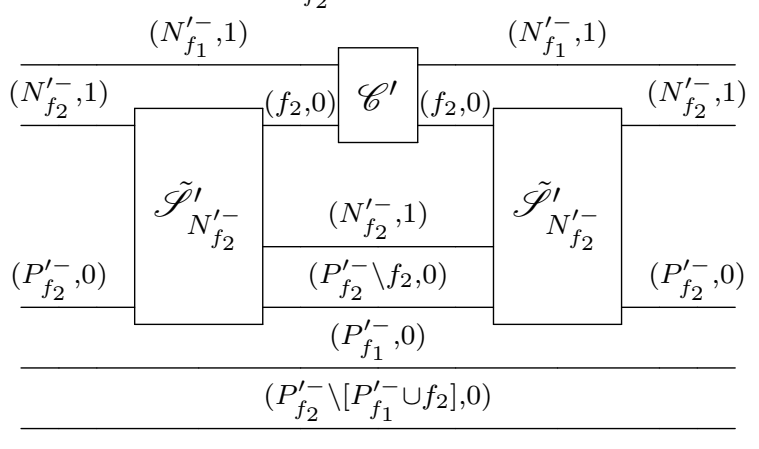

$$
=\frac{\left(P_{f_{1}}^{\prime-} \cup P_{f_{2}}^{\prime-}, 0\right)}{\frac{\left(N_{f_{1}}^{\prime-}, 1\right)}{\left(N_{f_{2}}^{\prime-}, 1\right)} \mathscr{C}^{\prime \prime} \frac{\left(N_{f_{1}}^{\prime-}, 1\right)}{\left(N_{f_{2}}^{\prime-}, 1\right)}},
$$

where on the l.h.s. we reversed the ordering of the wires for the sake of simplicity of the diagram. Overall, considering that $\tilde{\mathscr{S}}_{N_{f_{1}}^{\prime-} \cup N_{f_{2}}^{\prime-}}^{\prime}=$ $\tilde{\mathscr{S}}_{N_{f_{1}}^{\prime}}^{\prime} \tilde{\mathscr{S}}_{N_{f_{2}}^{\prime-}}^{\prime}=\tilde{\mathscr{S}}_{N_{f_{2}}^{\prime-}}^{\prime} \tilde{\mathscr{S}}_{N_{f_{1}}^{\prime-}}^{\prime}$, we then proved Eq. (110) for the local rule $\tilde{\mathscr{S}}_{f}^{\prime}$ on the neighbourhood scheme given by the graph $\Gamma\left(H, \phi\left(S_{+}\right)\right)$. In the second case, let $R=f_{1}=\phi\left(g_{1}\right), S=$ $f_{2}=\phi\left(g_{2}\right)$. The informations that matter are whether one can choose $g_{1}, g_{2}$ so that $f_{2} \in N_{f_{1}}^{\prime-}$ iff $g_{2} \in N_{g_{1}}^{-}$, and $\left|P_{f_{1}}^{\prime-} \cap N_{f_{2}}^{\prime+}\right|=\left|P_{g_{1}}^{-} \cap N_{g_{2}}^{+}\right|$. Again by lemma 66 , we know that the answer is positive. Then, also for $|R|=|S|=1$, since Eq. (110) holds for $\mathscr{C} \in \llbracket \mathrm{A}_{(\tilde{R}, 0) \cup(\tilde{S}, 1)}^{(G)} \rightarrow \mathrm{A}_{(\tilde{R}, 0) \cup(\tilde{S}, 1)}^{(G)} \rrbracket$, and

$$
\mathrm{A}_{(\tilde{R}, 0) \cup(\tilde{S}, 1)}^{(G)} \cong \mathrm{A}_{(R, 0) \cup(S, 1)}^{(H)}, \quad \tilde{\mathscr{S}}^{\prime}=\mathscr{S}^{\prime},
$$

we conclude that Eq. (110) holds also for $\mathscr{C} \in$ $\llbracket \mathrm{A}_{(R, 0) \cup(S, 1)}^{(H)} \rightarrow \mathrm{A}_{(R, 0) \cup(S, 1)}^{(H)} \rrbracket$. The argument for $|R|=0,|S|=2$ is the same as for $|R|=2$, $|S|=0$. For the converse, one can follow the same argument as for the direct statement. The only differences are that in the case $|R|=2$ and $|S|=0$ one might have $g_{2} \notin P_{g_{1}}^{-}$while $f_{2}=\phi\left(g_{2}\right) \in \phi\left(P_{g_{1}}^{-}\right)=P_{f_{2}}^{\prime-}$, and in the case $|R|=|S|=1$ it might happen that $g_{2} \notin N_{g_{1}}^{-}$ while $f_{2} \in N_{f_{1}}^{\prime-}$. By lemma 67 , the first case can occur only if $N_{g_{1}}^{+} \cap N_{g_{2}}^{+}=P_{g_{1}}^{-} \cap P_{g_{2}}^{-}=\emptyset$, while by lemma 66 the second case occurs only if $P_{g_{1}}^{-} \cap N_{g_{2}}^{+}=\emptyset$. In both cases, the different structure of the neighbourhoods between $G$ and $H$ does not preclude the derivation of Eq. (110) in the case of $G$ from the same identity for the case of $H$, as one can immediately realise by direct inspection of Eq. (110). 
Theorem 12 (Wrapping lemma). For a theory of type 1, under the hypotheses of lemma 66, the local rule $\left(N^{+}, \mathrm{A}, \mathscr{S}^{\prime}\right)$ defines a cellular automaton $(G, \mathrm{~A}, \mathscr{V})$ on the Cayley graph $\Gamma\left(G, S_{+}\right)$ if and only if the same local rule defines a cellular automaton $(H, \mathrm{~A}, \mathscr{W})$ on the Cayley graph $\Gamma\left(H, \phi\left(S_{+}\right)\right)$. The same is true of a theory of type 2, under the hypotheses of lemma $6 \%$.

Proof. The thesis now easily follows from lemmas 68 and 69, and theorem 10.

The above result allows us, at least in theories of the two types considered so far, and for CAs on Cayley graphs of groups with finite quotients satisfying the hypotheses of lemmas 66 or 67 , to reduce the classification of CAs on graphs of infinite groups to that of CAs on finite groups, which is in principle a much easier task. Moreover, once a suitable finite quotient is found, one can simulate the evolution of a finite region and for a finite number of steps considering the same evolution on the finite wrapped graph instead of having to consider the actual infinite graph.

\section{Examples}

\subsection{Classical case}

Classical systems d can be classified in terms of the number $d$ of pure states $\rho_{y}, y=1, \ldots, d$ which coincides with the dimension of the state space $d=\operatorname{dim} \llbracket \mathrm{d} \rrbracket_{\mathbb{R}}$. All the pure states of a classical system are jointly discriminable by atomic effects $a_{x}, x=1, \ldots, d$, i.e. $\left(a_{x} \mid \rho_{y}\right)=\delta_{x y}$. For the sake of simplicity, we will restrict attention to classical computation, i.e. the sub-theory of classical theory where there is only one kind of non-trivial elementary system, the bit having $d=2$, and all other systems are composite systems of $n$ bits, having dimension $d=2^{n}$. Every effect in $\llbracket \overline{\mathrm{A}}_{G} \rrbracket_{Q}$ for an infinite system $|G|=\infty$ can be decomposed as a series of effects corresponding to finite, arbitrarily large bit strings $b_{R}$, with

$$
b_{R}:=\left[a_{R}\right], \quad a=a_{b_{r_{1}}} \otimes a_{b_{r_{2}}} \otimes \ldots \otimes a_{b_{r_{|R|} \mid}},
$$

with $R=\left\{r_{1}, r_{2}, \ldots, r_{|R|}\right\}$. In this case, let $b_{R}$ denote a local effect. A global rule $\mathscr{V}$ can be specified by the input/output relation $\mathscr{V}^{\dagger} b_{\mathrm{R}}=b_{R^{\prime}}^{\prime}$. However, in the extensive literature about classical cellular automata - see e.g. [59] for reference, though we cannot even try to provide an exhaustive account of the relevant bibliography - it is customary to express $\mathscr{V}$ through its local rule, which is specified by its action on local states. The only point we want to discuss here is the apparent clash of our general wrapping lemma with results in the literature. In particular, in Ref. [31] a counterexample to the classical version of the wrapping lemma is provided, referring to [48]. The local rule for the counterexample is the following. Let $G=\mathbb{Z}_{r}$ with $r \notin 3 \mathbb{Z}$. The rule defined by

$$
b_{j}^{\prime}=b_{j \ominus_{r} 1} \oplus b_{j} \oplus b_{j \oplus_{r} 1}
$$

What we claim here is that, according to our notion of neighbourhood based on definition 36, the above automaton has a neighbourhood system where $N_{i}^{+}$is actually much larger than $\left\{j \ominus_{r} 1, j, j \oplus_{r} 1\right\}$. Indeed, if one considers the action of $\mathscr{V}$ on the algebra of transformations instead of its action on states, one can easily realise that $\mathrm{N}_{i}^{+}=G$ for every $i$. However, by checking the conditions for no-signalling, it is easy to realise that the rule in Eq. (117) only signals to sites $i \ominus 1, i$, and $i \oplus 1$. Thus, while no-signalling is necessary for no causal influence, the converse is not true.

We remark that the definition of causal influence that we give, based on transformations, is inspired by the quantum definition, which indeed involves the generators of the local algebra, and thus the Kraus representatives of transformations rather than the mere set of local states. In this respect, the notion of neighbourhood used in the literature on $\mathrm{CA}$ is different from the quantum one, and we point to this relevant difference as the responsible for the apparent incompatibility discussed in Ref. [31]. The subject was extensively studied in Ref. [60] and later in Ref.[61], where the authors maintain the notion of neighbourhood for classical CA based on the signalling condition, and prove a bound on the mismatch between the classical neighbourhood of a given $\mathrm{CA}$ and the neighbourhood of the quantum version of the same CA.

\subsection{Quantum case}

Quantum cellular automata represent now a widely studied field of research. After the very general analysis of Refs. [31, 32, 33, 61], there are various results in the literature about quantum 
cellular automata. Here we cite the very general classification of Ref. [62, 63], while for extensive reviews we refer to Refs. [64, 65].

\subsection{Fermionic case}

For the presentation of fermionic theory as an OPT we refer to [36, 37]. The Fermionic systems are fully specified by the algebra $\mathcal{A}$ generated by local field operators $\psi_{i}$. In fact, $\mathcal{A}$ is the algebra of field operator polynomials. This algebra is $\mathbb{Z}_{2}$-graded, with two modules $\mathcal{A}_{E}$ and $\mathcal{A}_{O}$, given by the span of even and odd polynomials, respectively. No combination of even and odd polynomials is allowed. As in all quantum theories, $\mathcal{A}$ provides a simplified representation in terms of Kraus operators of the algebra of transformations, which is not graded, but rather reducible to a direct sum. Linear combinations of transformations indeed do not correspond to linear combinations of the corresponding Kraus operators. The local systems for a site $g$ of a cellular automaton in this case are registers made of one or more local Fermionic modes. Local field operators are then labelled $\psi_{i, g}$, where $g \in G$ and $i \in J_{g}=\left\{1, \ldots, n_{g}\right\},\left|J_{g}\right|$ representing the size of the register at site $g$. Since the local algebra $\mathcal{A}_{g}$ is finitely generated, the locality requirement implies that $\mathscr{V}$ is given by a local rule, which in turn is completely specified by its action on the local generators $\psi_{i, g}$ for every $g \in G$. Thus, one has

$$
=\sum_{\substack{\mathbf{g} \in\left(N_{f}^{+}\right)^{\times k} \\ \mathbf{j} \in J_{\mathbf{g}} \\ \mathbf{s}, \mathbf{t} \in\{0,1\}^{*}}} T_{i, f}^{(\mathbf{g}, \mathbf{j}, \mathbf{s}, \mathbf{t})} \psi_{j_{1}, g_{1}}^{s_{j_{1}}} \psi_{j_{1}, g_{1}}^{\dagger t_{j_{1}}} \ldots \psi_{j_{k}, g_{k}}^{s_{k}} \psi_{j_{k}, g_{k}}^{\dagger t_{i_{k}}},
$$

where $J_{\mathrm{g}}:=\times_{g \in N_{f}^{+}} J_{g}$. Most of the automata studied so far in the literature are linear, namely

$$
\mathscr{V}\left(\psi_{i, f}\right)=\sum_{\substack{g \in N_{f}^{+} \\ j \in J_{g}}} T_{i, f}^{j, g^{\prime}} \psi_{j, g} .
$$

Remarkable exceptions are represented by Refs. [44, 66, 45]. In particular, in Ref. [45] the full classification of Fermionic cellular automata with $\left|J_{g}\right|=1$ on Cayley graphs of $\mathbb{Z}_{2} \times \mathbb{Z}_{2}$ and $\mathbb{Z}$ was carried out.

As regards Fermionic cellular automata, we would like to remark that, despite Fermionic theory being of type 2, they satisfy the wrapping lemma in the form of lemma 68, instead of lemma 69. This is due to the fact that Fermionic transofrmations, like quantum transformations, admit a Kraus decomposition, and can thus be represented through Kraus operators instead of completely positive maps. It turns out that local Fermionic field operators $\psi_{j, g}$ and $\psi_{j, g}^{\dagger}$ provide a basis for the full algebra of Fermionic operators. Thus, in the Fermionic case, if Eq. (110) is satisfied for local transformations, it is always satisfied. Real quantum theory, which shares many features with Fermionic theory, is different in this respect, and we conjecture that a counterexample to the thesis of lemma 68 can be found in the latter case.

\section{Conclusion}

Summarising the content of the manuscript, we defined the composition of denumerably many systems from an OPT, starting from the space of quasi-local effects, and defining quasi-local transformations. We then define states of such system in terms of suitable functionals on the space of effects. Among these, there are some that can be interpreted as the result of local preparations, and that we deem quasi-local states.

We defined global update rules for finite or infinite systems, and proved a generalisation of a block-decomposition theorem for GURs. We then analysed those GURs that are homogeneous. The definition of homogeneity is a big chapter on its own, and deserves a careful treatment. As a consequence of the definition, we proved that for homogenous GURs the memory array is organised as the Cayley graph of a group. We then defined locality for GURs, and studied its consequences in detail.

In the last section we used homogeneity and locality to define cellular automata, and proved the wrapping lemma under very general assumptions.

The theory developed here is of fundamental importance for an approach to physical laws as information-processing algorithms, and will be used for the construction of statistical mechanics in post-quantum theories, as well as the reconstruction of dynamical laws in space-time beyond the now established approach based on quantum walks, thus encompassing interacting quantum and post-quantum field theories. 


\section{Acknowledgments}

This publication was made possible through the support of a grant from the John Templeton Foundation under the project ID\#60609 Causal Quantum Structures. The opinions expressed in this publication are those of the authors and do not necessarily reflect the views of the John Templeton Foundation. The author wishes to thank G. M. D'Ariano, A. Bisio, A. Tosini, and F. Buscemi for many inspiring discussions. Suggestions about the terminology by G. Chiribella are also acknowledged that were useful to sharpen the notion that is now defined as causal influence. A particular thank to J. Barrett for fruitful conversations about various facets of causal influence and signalling, and to M. Erba for his help in tuning the definitions and dissipating misconceptions on the same subject.

\section{A Identification of the sup- and oper- ational norm for effects}

We show that, under the hypothesis of assumption (16), one has $\|a\|_{\text {op }}=\|a\|_{\text {sup }}$ for every $a \in \llbracket \overline{\mathrm{A}} \rrbracket_{\mathbb{R}}$. Indeed, if $\llbracket \overline{\mathrm{A}} \rrbracket_{+}=\llbracket \mathrm{A} \rrbracket_{+}^{*}$, then, by theorem 5 , one has

$$
\llbracket \overline{\mathrm{A}} \rrbracket=\left\{a \in \llbracket \overline{\mathrm{A}} \rrbracket_{\mathbb{R}} \mid 0 \leq(a \mid \rho),(e-a \mid \rho), \forall \rho \in \llbracket \mathrm{A} \rrbracket\right\} .
$$

First of all, one can rephrase the above condition as

$$
\llbracket \overline{\mathrm{A}} \rrbracket=\left\{a \in \llbracket \overline{\mathrm{A}} \rrbracket_{\mathbb{R}} \mid 0 \leq(a \mid \rho),(e-a \mid \rho), \forall \rho \in \llbracket \mathrm{A} \rrbracket_{1}\right\} .
$$

Indeed, since $\llbracket A \rrbracket_{1} \subseteq \llbracket A \rrbracket$, the set on the 1.h.s. in Eq. (118) is clearly a subset of that on the l.h.s. in Eq. (119). Moreover, since every state $\rho \in \llbracket \mathrm{A} \rrbracket$ is proportional by a constant $\kappa \in[0,1]$ to a deterministic one $\rho_{0} \in \llbracket \mathrm{A} \rrbracket_{1}$ (see remark 1 ), if $0 \leq(a \mid \sigma),(e-a \mid \sigma)$ for all $\sigma \in \llbracket \mathrm{A} \rrbracket_{1}$, then $0 \leq(a \mid \rho),(e-a \mid \rho)$ for all $\rho \in \llbracket \mathrm{A} \rrbracket$. Then, by the identity in Eq. (119), one can identify the set $\llbracket \overline{\mathrm{A}} \rrbracket$ as the subset of $\llbracket \overline{\mathrm{A}} \rrbracket_{\mathbb{R}}$ containing only those functionals $a$ on $\llbracket \mathrm{A} \rrbracket_{\mathbb{R}}$ such that $0 \leq(a \mid \rho)$ and $0 \leq 1-(a \mid \rho)$ for $\rho \in \llbracket \mathrm{A} \rrbracket_{1}$, and, in turn, the above condition is equivalent to

$$
\llbracket \overline{\mathrm{A}} \rrbracket=\left\{a \in \llbracket \overline{\mathrm{A}} \rrbracket_{\mathbb{R}} \mid 0 \leq(a \mid \rho) \leq 1, \forall \rho \in \llbracket \mathrm{A} \rrbracket\right\} .
$$

Now, we remind that $\lambda \in J(a)$ if and only if $\lambda e \pm a \succeq 0$. Under our hypotheses, this means that $\lambda \pm(a \mid \rho) \geq 0$ for every $\rho \in \llbracket \mathrm{A} \rrbracket$. Thus, $|(a \mid \rho)| \leq \lambda$ for every $\rho \in \llbracket \mathrm{A} \rrbracket$. Finally, we can conclude that $\|a\|_{\text {op }} \leq\|a\|_{\text {sup }}$. On the other hand, if $\|a\|_{\text {op }}<\|a\|_{\text {sup }}$, then there exists $\varepsilon>0$ such that for every $\rho \in \llbracket \mathrm{A} \rrbracket$ one has $|(a \mid \rho)| \leq\|a\|_{\text {sup }}-\varepsilon$. In particular, this is true for $\rho_{1} \in \llbracket \mathrm{A} \rrbracket_{1}$. Consequently

$$
|(a \mid \rho)|=\kappa\left|\left(a \mid \rho_{1}\right)\right| \leq \kappa\left(\|a\|_{\text {sup }}-\varepsilon\right) \forall \rho \in \llbracket \mathrm{A} \rrbracket,
$$

where $0 \leq \kappa \leq 1$ and $\rho=\kappa \rho_{1}$. Then $(e \mid \rho)=\kappa$, and

$$
\left(\left\{\|a\|_{\text {sup }}-\varepsilon\right\} e \pm a \mid \rho\right) \geq 0, \forall \rho \in \llbracket \mathrm{A} \rrbracket .
$$

Finally, this implies that $\|a\|_{\text {sup }}-\varepsilon \in J(a)$, which is absurd. Then, $\|a\|_{\text {sup }}=\|a\|_{\text {op }}$.

\section{B Quasi-local states}

We start considering arbitrary finite disjoint partitions of the system $A_{G}$. Let $B:=\left\{B_{i}\right\}_{i=1}^{\infty}$ denote a disjoint partition of the set $G$ into finite blocks, i.e. $0<\left|B_{i}\right|<\infty$ for every $i \in \mathbb{N}$. The set of such partitions (quotiented by the irrelevant ordering of regions) is denoted by $B$.

Definition 51. For a given partition $B \in \mathrm{B}$, we define the set of finite $B$-regions of $G$ as

$$
\mathrm{R}^{(G, B)}:=\left\{R \subseteq G \mid R=\bigcup_{j=1}^{k} B_{i_{j}} ; 0 \leq k<\infty\right\}
$$

and the set of $B$-regions of $G$ as

$$
\overline{\mathrm{R}}^{(G, B)}:=\left\{R \subseteq G \mid R=\bigcup_{j=1}^{k} B_{i_{j}} ; 0 \leq k \leq \infty\right\}
$$

where for $k=0$ we define

$$
\bigcup_{j=1}^{0} B_{i_{j}}:=\emptyset
$$

Given $R=\bigcup_{j=1}^{k} B_{i_{j}}$ in $\overline{\mathrm{R}}^{(G, B)}$, let $\mathbb{N}_{R}^{(B)}:=$ $\left\{i_{j}\right\}_{j=1}^{k}$.

Remark 10. Clearly, $\mathrm{R}^{(G, B)} \subseteq \overline{\mathrm{R}}^{(G, B)}$. It is also trivial to prove that $\overline{\mathrm{R}}^{(G, B)}$ and $\mathrm{R}^{(G, B)}$ are closed under $\cup, \cap, \backslash$. 
Given a finite region $R \in \mathrm{R}^{(G)}$, for any partition $B \in \mathrm{B}$ it is possible to cover $R$ with regions of $B$.

Definition 52. Given $B \in \mathrm{B}$, the cover in $B$ of a region $R \subseteq G$, denoted $B(R)$, is the minimal $B$-region $C=\bigcup_{j=1}^{k} B_{i_{j}}$ such that $R \subseteq C$.

The above notion of cover is well defined, as proved by the following lemma.

Lemma 70. Let $R \subseteq G$. The cover $B(R)$ in $B$ of $R$ exists and is unique.

Proof. First of all, the set of $B$-regions $S \in$ $\overline{\mathrm{R}}^{(G, B)}$ such that $R \subseteq S$ is not empty, since $R \subseteq G \in \overline{\mathrm{R}}^{(G, B)}$. We just need to prove that, having defined $\mathrm{C}(R, B):=\left\{S \in \overline{\mathrm{R}}^{(G, B)} \mid R \subseteq S\right\}$, there is always a unique minimal element $B(R) \in$ $\mathrm{C}(R, B)$, i.e. such that no $B$-region strictly contained in $B(R)$ covers $R$. As to existence, let $h \in R$. Then $h \in B_{l_{h}}$ for some $l_{h}$. One can easily realise that $R \subseteq C:=\bigcup_{h \in R} B_{l_{h}}$. Moreover, if we remove a given $B$-region $B_{l_{h}}$ from $C$, and $h \in B_{l_{h}}$, then $h \notin C \backslash B_{l_{h}}$, thus $C$ is a minimal cover of $R$ in $B$. As to uniqueness, suppose that there are two different minimal covers of $R$ in $B$, say $C_{1}$ and $C_{2}$. In this case, it is not restrictive to suppose that there is $h \in G$ such that $h \in C_{1}$ and $h \notin C_{2}$. Let $B_{i} \in B$ be the set such that $h \in B_{i}$. One has $B_{i} \subseteq C_{1}$ and $B_{i} \cap C_{2}=\emptyset$. Since $R \subseteq C_{2}$, we can conclude that $R \cap B_{i}=\emptyset$, and thus $R \subseteq\left(C_{1} \backslash B_{i}\right) \in \mathrm{C}(R, B)$. Thus, $C_{1}$ cannot be minimal in $\mathrm{C}(R, B)$.

The cover $B(R)$ can also be thought of as the intersection of all covers of $R$ in $B$.

Lemma 71. The cover $B(R)$ of a finite region $R$ is finite.

Proof. Every element $g_{k} \in R$ belongs to some $B_{i_{k}} \subseteq B$, where $k \mapsto i_{k}$ is generally not injective. It is then clear that $R \subseteq \bigcup_{k=1}^{|R|} B_{i_{k}}$, and thus $B(R) \subseteq \bigcup_{k=1}^{|R|} B_{i_{k}}$.

Given one partition $B \in \mathrm{B}$, we can now define the set of states that differ on finitely many blocks from a given assignment of states of blocks in $B$. First, we define the reference state as follows.

Definition 53 (Reference local state). Given a partition $B \in \mathrm{B}$, a reference local state over $B$ is a map

$$
\rho_{0}: \mathbb{N} \rightarrow \bigsqcup_{j \in \mathbb{N}} \llbracket \mathrm{A}_{B_{j}} \rrbracket_{1}:: j \mapsto \rho_{0 j} \in \llbracket \mathrm{A}_{B_{j}} \rrbracket_{1} .
$$

A reference local state allows one to attribute a state to any finite region $R \in \mathrm{R}^{(G)}$ by the following procedure. First, let us consider the $B$-cover $B(R)$ of $R$. Then, the state of $B(R)$ is given by

$$
\rho_{0}[B(R)]:=\bigotimes_{i_{j} \in \mathbb{N}_{B(R)}^{(B)}} \rho_{0 i_{j}} .
$$

Finally, we discard all the extra systems in the region $B(R) \backslash R$.

$$
\rho_{0 \mid R}:=\rho_{0}[B(R)]_{\mid R} .
$$

The above construction is then formalised in the following definition.

Definition 54. The state of a finite region $R \in$ $\mathrm{R}^{(G, B)}$ in the reference local state $\rho_{0}$ is defined as

$$
\begin{aligned}
& \rho_{0 \mid R}^{R}:=\rho_{0}[B(R)] \frac{R}{B(R) \backslash R}, e \\
& \rho_{0 \mid \emptyset}:=1 .
\end{aligned}
$$

Given a partition $B$ and a reference local state $\rho_{0}$, let us now define the set of primitive local states as follows.

Definition 55. The set of primitive local states is

$$
\operatorname{Pre} \llbracket \mathrm{A}_{G} \rrbracket_{\mathbb{R}}^{(B)}:=\left\{(\sigma, R) \mid \sigma \in \llbracket \mathrm{A}_{R} \rrbracket_{\mathbb{R}} ; R \in \mathrm{R}^{(G, B)}\right\} .
$$

This set collects all the generalised local preparations of finite regions of $B$. As in the case of effects, we introduce the simplified notation $\sigma_{S}:=(\sigma, S)$. A choice of reference local state $\rho_{0}$, allows us to interpret a given element $\sigma_{S}$ of Pre $\llbracket \mathrm{A}_{G} \rrbracket_{\mathbb{R}}^{(B)}$ as a preparation of the region $S$ in the state $\sigma$. Thus, the element $\sigma_{S}$ allows one to attribute a generalised state $\tau \in \llbracket \mathrm{A}_{T} \rrbracket_{\mathbb{R}}$ to any region $T \in \mathrm{R}^{(G)}$ as follows (see fig. 3)

$$
\tau:=\sigma_{\mid S \cap T} \otimes \rho_{0 \mid(T \backslash S)}
$$

Definition 56. Given a reference local state $\rho_{0}$ over $B$ we define the following equivalence relation in $\operatorname{Pre} \llbracket \mathrm{A}_{G} \rrbracket_{\mathbb{R}}^{(B)}$

$$
\sigma_{S} \sim_{\rho_{0}} \tau_{T} \Leftrightarrow\left\{\begin{array}{l}
\sigma=\nu \otimes \rho_{0 \mid(S \backslash T)}, \\
\tau=\nu \otimes \rho_{0 \mid(T \backslash S)}
\end{array}\right.
$$

for some $\nu \in \llbracket \mathrm{A}_{S \cap T} \rrbracket \rrbracket \mathbb{R}$. 


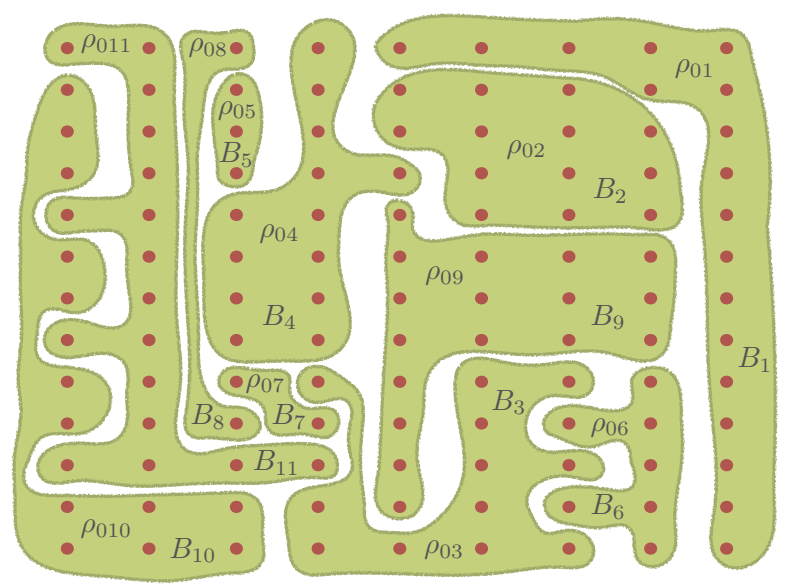

(a)

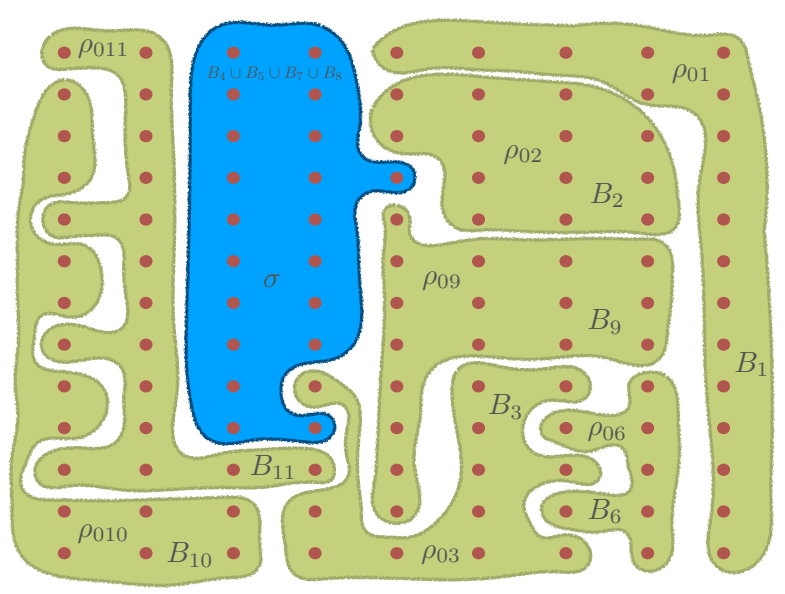

(b)

Figure 3: An illustrative example of a reference local state $\rho_{0}$ (a) for a partition of a finite set $G$ into 11 regions, along with a primitive local state $\sigma_{R}$ (b) with $R=B_{4} \cup B_{5} \cup B_{7} \cup B_{8}$. The dots represent the elements of $G$.
The symbol $\sigma_{S \rho_{0}}$ will denote the equivalence class of $\sigma_{S} \in \operatorname{Pre} \llbracket \mathrm{A}_{G} \rrbracket_{\mathbb{R}}^{(B)}$ under the relation $\sim_{\rho_{0}}$. Notice that in Eq. (123), thanks to closure of $\mathrm{R}^{(G, B)}$ under set difference, one has $(S \backslash T),(T \backslash$ $S) \in \mathrm{R}^{(G, B)}$, and thus the definition of the equivalence relation $\sim_{\rho_{0}}$ involves exclusively $B$-regions. Moreover, all the regions involved in the definition are finite.

Lemma 72. Let $\sigma_{S} \in \operatorname{Pre} \llbracket \mathrm{A}_{G} \rrbracket_{\mathbb{R}}^{(B)}$. Then for every $R \in \mathrm{R}^{(G, B)}$ such that $R \cap S=\emptyset$ one has $(\sigma \otimes$ $\left.\rho_{0 \mid R}\right)_{S \cup R} \in \operatorname{Pre} \llbracket \mathrm{A}_{G} \rrbracket_{\mathbb{R}}^{(B)}$ and $\left(\sigma \otimes \rho_{0 \mid R}\right)_{S \cup R} \sim_{\rho_{0}}$ $\sigma_{S}$.

Proof. First of all, as $\mathrm{R}^{(G, B)}$ is closed under union, clearly $S \cup R \in \mathrm{R}^{(G, B)}$, and thus $(\sigma \otimes$ $\left.\rho_{0 \mid R}\right)_{S \cup R} \in \operatorname{Pre} \llbracket \mathrm{A}_{G} \rrbracket_{\mathbb{R}}^{(B)}$. Now, $S \cap(S \cup R)=S$, and thus the condition in Eq. (123) is satisfied for $T=S \cup R, T \backslash S=R, S \cap T=S, \nu=\sigma$, $\rho_{0 \mid(T \backslash S)}=\rho_{0 \mid R}$, and $\rho_{0 \mid(S \backslash T)}=1$.

We now provide a way to identify a canonical representative of the equivalence class $\sigma_{R \rho_{0}}$ defined as follows.

Definition 57. The minimal representative $\tilde{\sigma}_{R_{\sigma}}$ of the equivalence class $\sigma_{R \rho_{0}}$ is defined through

$$
R_{\sigma}:=\bigcap_{S \in \mathrm{R}_{(\sigma, R)}} S, \quad \tilde{\sigma}_{R_{\sigma}} \sim_{\rho_{0}} \sigma_{R}
$$

where

$\mathrm{R}_{(\sigma, R)}:=\left\{S \in \mathrm{R}^{(G, B)} \mid \exists \tau \in \llbracket \mathrm{A}_{S} \rrbracket_{\mathbb{R}}: \tau_{S} \sim_{\rho_{0}} \sigma_{R}\right\}$.

Lemma 73. The minimal representative exists and is unique.

For the proof, see the analogous lemma 9.

Definition 58. The set of generalised local states over $B$ upon $\rho_{0}$ is

$$
\llbracket \mathrm{A}_{G} \rrbracket_{\rho_{0} L \mathbb{R}}^{(B)}:=\operatorname{Pre} \llbracket \mathrm{A}_{G} \rrbracket_{\mathbb{R}}^{(B)} / \sim_{\rho_{0}} .
$$

Its elements will be denoted by symbols like $\rho_{S \rho_{0}}$.

We now define linear combinations of local states.

Definition 59. Let $\sigma_{S \rho_{0}}, \tau_{T \rho_{0}} \in \llbracket \mathrm{A}_{G} \rrbracket_{\rho_{0} L \mathbb{R}}^{(B)}$, and $a \in \mathbb{R}$. Then we have

$$
\begin{aligned}
& a\left(\sigma_{S \rho_{0}}\right):= \begin{cases}(a \sigma)_{S \rho_{0}} & a \neq 0 \\
0_{\emptyset \rho_{0}} & a=0,\end{cases} \\
& \sigma_{S \rho_{0}}+\tau_{T \rho_{0}}:=\nu_{S \cup T \rho_{0}}, \\
& \nu:=\sigma \otimes \rho_{0 \mid(T \backslash S)}+\rho_{0 \mid(S \backslash T)} \otimes \tau .
\end{aligned}
$$


We will denote by $R_{\sigma+\tau}$ the region where the minimal representative of the class $\sigma_{S \rho_{0}}+\tau_{T \rho_{0}}$ differs from $\rho_{0}$. As in the case of effects, it is not always true that $R_{\sigma+\tau}=R_{\sigma} \cup R_{\tau}$. As an example, consider $\sigma=\alpha_{i_{1}} \otimes \beta_{i_{2}}$ and $\tau=\alpha_{i_{1}} \otimes\left(\rho_{0 i_{2}}-\beta_{i_{2}}\right)$, with $\alpha_{i_{1}} \neq \rho_{0 i_{1}}, \beta_{i_{2}} \neq \rho_{0 i_{2}}$, and $R_{\sigma}=R_{\tau}=$ $B_{i_{1}} \cup B_{i_{2}}$. Then $\sigma+\tau=\alpha_{i_{1}} \otimes \rho_{0 i_{2}}$, and clearly $R_{\sigma+\tau}=B_{i_{1}}$, which is strictly included in $R_{\sigma}=$ $R_{\tau}=R_{\sigma} \cup R_{\tau}$.

Definition 60. The set of local states over $B$ upon $\rho_{0}$ is

$$
\llbracket \mathrm{A}_{G} \rrbracket_{\rho_{0} L}^{(B)}:=\left\{\sigma_{S \rho_{0}} \in \llbracket \mathrm{A}_{G} \rrbracket_{\rho_{0} L \mathbb{R}}^{(B)} \mid \sigma \in \llbracket \mathrm{A}_{S} \rrbracket\right\} .
$$

An element $\sigma_{S \rho_{0}} \in \llbracket \mathrm{A}_{G} \rrbracket_{\rho_{0} L \mathbb{R}}^{(B)}$ is a local state over $B$ upon $\rho_{0}$. A subset of special interest is that of deterministic local states over $B$ upon $\rho_{0}$, defined as

$$
\llbracket \mathrm{A}_{G} \rrbracket_{\rho_{0} L 1}^{(B)}:=\left\{\sigma_{S \rho_{0}} \in \llbracket \mathrm{A}_{G} \rrbracket_{\rho_{0} L}^{(B)} \mid \sigma \in \llbracket \mathrm{A}_{S} \rrbracket_{1}\right\} .
$$

We now characterise the vector space of generalised states as follows.

Lemma 74. The set of generalized local states in $B$ upon $\rho_{0}$ equipped with the operations defined in Def. 59 is a real vector space. It is the space of finite real combinations of deterministic local states in $B$ upon the same state $\rho_{0}$ :

$$
\llbracket \mathrm{A}_{G} \rrbracket_{\rho_{0} L \mathbb{R}}^{(B)}=\operatorname{Span}_{\mathbb{R}}\left(\llbracket \mathrm{A}_{G} \rrbracket_{\rho_{0} L 1}^{(B)}\right),
$$

The proof is trivial and we omit it.

We now show that quasi-local states are bounded linear functionals on $\llbracket \overline{\mathrm{A}}_{G} \rrbracket_{Q \mathbb{R}}$. We start considering the pairing of local states $\llbracket \mathrm{A}_{G} \rrbracket_{\rho_{0} L \mathbb{R}}^{(B)}$ with local effects $\llbracket \overline{\mathrm{A}}_{G} \rrbracket_{L \mathbb{R}}$, defined as follows.

Definition 61. Every $\sigma_{S \rho_{0}} \in \llbracket \mathrm{A}_{G} \rrbracket_{\rho_{0} L \mathbb{R}}$ identifies a functional on $\llbracket \overline{\mathrm{A}}_{G} \rrbracket_{L \mathbb{R}}$ as follows. For $a_{R} \in \llbracket \overline{\mathrm{A}}_{G} \rrbracket_{L \mathbb{R}}$ let

$$
\left(a_{R} \mid \sigma_{S \rho_{0}}\right):=\sigma_{\rho_{0 \mid R \backslash S}} R \backslash S \text {. }
$$

We now prove that the above pairing is welldefined, namely it does not depend on the representative in the class $\sigma_{S \rho_{0}}$, nor on the representative in the class $a_{R}$. This is done in the next two lemmas.

Lemma 75. For every $a_{R} \in \llbracket \overline{\mathrm{A}}_{G} \rrbracket_{L \mathbb{R}}$ the result of Eq. (127) is independent of the choice of representative $\tau_{T \rho_{0}}$ in the class $\sigma_{S \rho_{0}}$.
Proof. By hypothesis, there exists $\nu \in \llbracket \mathrm{A}_{S \cap T} \rrbracket \mathbb{R}$ such that

$$
\sigma=\nu \otimes \rho_{0 S \backslash T}, \quad \tau=\nu \otimes \rho_{0 T \backslash S} .
$$

Then we have

$$
\begin{aligned}
& \left(a_{R} \mid \sigma_{S \rho_{0}}\right)=\left(a \mid \nu_{\mid(S \cap T) \cap R} \otimes \rho_{0 \mid[(S \backslash T) \cap R] \cup(R \backslash S)}\right) \\
& \left(a_{R} \mid \tau_{T \rho_{0}}\right)=\left(a \mid \nu_{\mid(S \cap T) \cap R} \otimes \rho_{0 \mid[(T \backslash S) \cap R] \cup(R \backslash T)}\right) .
\end{aligned}
$$

A straightforward set-theoretic calculation gives that $[(S \backslash T) \cap R] \cup(R \backslash S)=[(T \backslash S) \cap R] \cup(R \backslash T)=$ $R \backslash(S \cap T)$, and thus $\left(a_{R} \mid \sigma_{S \rho_{0}}\right)=\left(a_{R} \mid \tau_{T \rho_{0}}\right)$.

On similar lines, we have the following result.

Lemma 76. For every partition $B \in \mathrm{B}$, every reference local state $\rho_{0}$ over $B$, and every local state $\tau_{T \rho_{0}} \in \llbracket \mathrm{A}_{G} \rrbracket_{\rho_{0} L \mathbb{R}}^{(B)}$, the result of $E q$. (127) is independent of the choice of representative $b_{S}$ in the class $a_{R}$.

Proof. If $\tilde{c}_{R_{c}}$ is the minimal representative of $a_{R}=b_{S}$, by Eq. (10), one has

$$
\begin{aligned}
& a=\tilde{c} \otimes e_{R \backslash R_{c}}, \\
& b=\tilde{c} \otimes e_{S \backslash R_{c}} .
\end{aligned}
$$

Now, by the defining equation 127 , we have that

$$
\begin{aligned}
& \left(a_{R} \mid \tau_{T \rho_{0}}\right)=\left(a \mid \tau_{\mid R \cap T} \otimes \rho_{0 \mid(R \backslash T)}\right) \\
= & \left(\tilde{c} \otimes e_{R \backslash R_{c}} \mid \tau_{\mid R \cap T} \otimes \rho_{0 \mid(R \backslash T)}\right) \\
= & \left(\tilde{c} \mid \tau_{\mid R_{c} \cap T} \otimes \rho_{0 \mid R_{c} \backslash T}\right),
\end{aligned}
$$

and similarly we obtain

$$
\left(b_{S} \mid \tau_{T \rho_{0}}\right)=\left(\tilde{c} \mid \tau_{\mid R_{c} \cap T} \otimes \rho_{0 \mid\left(R_{c} \backslash T\right)}\right) .
$$

The above results give us a hint that for every $B \in \mathrm{B}$ and every $\rho_{0}$, the space $\llbracket \mathrm{A}_{G} \rrbracket_{\rho_{0} L \mathbb{R}}^{(B)}$ is a submanifold of the space $\llbracket \mathrm{A}_{G} \rrbracket_{\mathbb{R}}$. We now complete the real vector spaces $\llbracket \mathrm{A}_{G} \rrbracket_{\rho_{0} L \mathbb{R}}^{(B)}$ to Banach spaces. For this purpose, we start equipping all spaces $\llbracket \mathrm{A}_{G} \rrbracket_{\rho_{0} L \mathbb{R}}^{(B)}$ with a norm.

Definition 62. Given an element $\sigma$ of the space $\llbracket \mathrm{A}_{G} \rrbracket_{\rho_{0} L \mathbb{R}}^{(B)}$, its norm is defined as

$$
\|\sigma\|_{B, \rho_{0}}:=\|\tilde{\sigma}\|_{\mathrm{op}}
$$

where $\|\cdot\|_{\mathrm{op}}$ denotes the operational norm on $\llbracket \mathrm{A}_{R_{\sigma}} \rrbracket \mathbb{R}$. 
Lemma 77. Given an element $\sigma$ of $\llbracket \mathrm{A}_{G} \rrbracket_{\rho_{0} L \mathbb{R}}^{(B)}$, for $\tau_{T}$ in the corresponding equivalence class, one has

$$
\|\sigma\|_{B, \rho_{0}}=\|\tau\|_{\mathrm{op}},
$$

where $\|\cdot\|_{\text {op }}$ denotes the operational norm on $\llbracket \mathrm{A}_{T} \rrbracket_{\mathbb{R}}$.

Proof. By definition of the relation $\sim_{\rho_{0}}$, and of minimal representative $\tilde{\sigma}_{R_{\sigma}}$ of $\sigma$, one has

$$
\tau=\tilde{\sigma} \otimes \rho_{0\left(T \backslash R_{\sigma}\right)} .
$$

Thus, $\|\tau\|_{\text {op }}=\left\|\tilde{\sigma} \otimes \rho_{0\left(T \backslash R_{\sigma}\right)}\right\|_{\text {op. }}$. Now, by lemma 3

$$
\|\tau\|_{\mathrm{op}}=\|\tilde{\sigma}\|_{\mathrm{op}}=\|\sigma\|_{B, \rho_{0}} .
$$

We can now show that vectors in $\llbracket \mathrm{A}_{G} \rrbracket_{\rho_{0} L \mathbb{R}}^{(B)}$ are bounded linear functionals on $\llbracket \overline{\mathrm{A}}_{G} \rrbracket_{Q \mathbb{R}}$.

Definition 63. Let $a=\left[a_{n R_{n}}\right] \in \llbracket \overline{\mathrm{A}}_{G} \rrbracket_{Q \mathbb{R}}$, and $\rho=\rho_{S \rho_{0}} \in \llbracket \mathrm{A}_{G} \rrbracket_{\rho_{0} L \mathbb{R}}$. We define

$$
(a \mid \rho):=\lim _{n \rightarrow \infty}\left(a_{n R_{n}} \mid \rho_{S \rho_{0}}\right) .
$$

With this definition, we can now prove that local states are actually bounded linear functionals on $\llbracket \overline{\mathrm{A}}_{G} \rrbracket_{Q \mathbb{R}}$.

Lemma 78. For $a=\left[a_{n R_{n}}\right] \in \llbracket \overline{\mathrm{A}}_{G} \rrbracket_{Q \mathbb{R}}$, and $\rho_{S \rho_{0}} \in \llbracket \mathrm{A}_{G} \rrbracket \rho_{\rho_{0} L \mathbb{R}}$, one has

$$
|(a \mid \rho)| \leq\|a\|_{\text {sup }}\|\rho\|_{B, \rho_{0}} .
$$

Proof. We start considering the case of a local effect $a_{R}$. In this case, us define

$$
\begin{aligned}
a_{0}:=\left(2\|a\|_{\text {sup }}\right)^{-1}\left(\|a\|_{\text {sup }} e_{R}+a\right) & \succeq 0, \\
a_{1}:=\left(2\|a\|_{\text {sup }}\right)^{-1}\left(\|a\|_{\text {sup }} e_{R}-a\right) & \succeq 0, \\
a_{0}+a_{1}=e_{R}, \quad\|a\|_{\text {sup }}\left(a_{0}-a_{1}\right) & =a,
\end{aligned}
$$

we have that

$$
\begin{aligned}
\left|\left(a_{R} \mid \rho_{S \rho_{0}}\right)\right| & =\left|\left(a_{R} \mid \rho_{\mid R \cap S} \otimes \rho_{0 \mid R \backslash S}\right)\right| \\
& =\|a\|_{\text {sup }}\left|\left(\left\{\bar{a}_{0}-\bar{a}_{1}\right\} \otimes e_{S \backslash R} \mid \rho \otimes \rho_{0 \mid R \backslash S}\right)\right| \\
& \leq\|a\|_{\text {sup }}\left\|\rho_{S \rho_{0}}\right\|_{B, \rho_{0}},
\end{aligned}
$$

where $\left(\bar{a}_{i} \mid:=\left(a_{i}\left|\mathscr{I}_{R \cap S} \otimes\right| \rho_{0 \mid R \backslash S}\right)\right.$. Let now $a=$ $\left[a_{n R_{n}}\right]$. Then we have

$$
\begin{aligned}
\mid\left(a_{n R_{n}}\right. & \left.-a_{m R_{m}} \mid \rho_{S \rho_{0}}\right) \mid \\
& \leq\left\|a_{n R_{n}}-a_{m R_{m}}\right\|_{\text {sup }}\left\|\rho_{S \rho_{0}}\right\|_{B, \rho_{0}},
\end{aligned}
$$

and thus actually $\left(a_{n R_{n}} \mid \rho_{S \rho_{0}}\right)$ is a Cauchy sequence. Finally, since for every $n \in \mathbb{N}$ one has

$$
\left|\left(a_{n R_{n}} \mid \rho_{S \rho_{0}}\right)\right| \leq\left\|a_{n}\right\|_{\text {sup }}\left\|\rho_{S}\right\|_{B, \rho_{0}},
$$

taking the limit for $n \rightarrow \infty$ on both sides we have that

$$
\left|\left(a \mid \rho_{S \rho_{0}}\right)\right| \leq\|a\|_{\text {sup }}\left\|\rho_{S \rho_{0}}\right\|_{B, \rho_{0}} .
$$

We now show that the definition in Eq. (129) is independent of the sequence $a_{n R_{n}}$ in the class of $a$.

Lemma 79. Let $a=\left[a_{n R_{n}}\right]=\left[b_{n R_{n}^{\prime}}\right] \in \llbracket \overline{\mathrm{A}}_{G} \rrbracket_{Q \mathbb{R}}$, and $\rho_{S \rho_{0}} \in \llbracket \mathrm{A}_{G} \rrbracket \rho_{0} L \mathbb{R}$. Then

$$
\lim _{n \rightarrow \infty}\left(a_{n} \mid \rho_{S \rho_{0}}\right)=\lim _{n \rightarrow \infty}\left(b_{n} \mid \rho_{S \rho_{0}}\right) .
$$

Proof. Remind that by definition, for every $\varepsilon$ there exists $n_{0}$ such that for $n \geq n_{0}$, it is $\left\|a_{n R_{n}}-b_{n R_{n}^{\prime}}\right\|_{\text {sup }} \leq \varepsilon$. Now, by lemma 78 , this implies that

$$
\left|\left(a_{n R_{n}}-b_{n R_{n}^{\prime}} \mid \rho_{S \rho_{0}}\right)\right| \leq \varepsilon\left\|\rho_{S \rho_{0}}\right\|_{B, \rho_{0}},
$$

and thus the thesis follows.

We finally close the vector space $\llbracket \mathrm{A}_{G} \rrbracket_{\rho_{0} L \mathbb{R}}^{(B)}$ by adding the limits of Cauchy sequences in the norm $\|\cdot\|_{B, \rho_{0}}$, thus obtaining a Banach space that we denote $\llbracket \mathrm{A}_{G} \rrbracket_{\rho_{0} Q \mathbb{R}}^{(B)}$. We start considering Cauchy sequences

$$
\sigma: \mathbb{N} \rightarrow \llbracket \mathrm{A}_{G} \rrbracket_{\rho_{0} \mathbb{R}}^{(B)}:: n \mapsto \sigma_{n R_{n} \rho_{0}} \in \llbracket \mathrm{A}_{G} \rrbracket_{\rho_{0} L \mathbb{R}},
$$

which make a real vector space $\llbracket \mathrm{A}_{G} \rrbracket_{\rho_{0} C \mathbb{R}}^{(B)}$, containing $\llbracket \mathrm{A}_{G} \rrbracket_{\rho_{0} L \mathbb{R}}^{(B)}$ as the subspace of constant sequences. We then quotient $\llbracket \mathrm{A}_{G} \rrbracket_{\rho_{0} C \mathbb{R}}^{(B)}$ by the usual equivalence relation $\cong$, obtaining complete normed real vector space.

Definition 64. The space of quasi-local states in $B$ upon $\rho_{0}$ is defined as the space $\llbracket \mathrm{A}_{G} \rrbracket_{\rho_{0} Q \mathbb{R}}^{(B)}:=$ $\llbracket \mathrm{A}_{G} \rrbracket_{\rho_{0} C \mathbb{R}}^{(B)} / \cong$.

Inside the new Banach space that we defined, we can single out a convex set of quasi-local states, corresponding to preparations that can be arbitrarily approximated by local protocols starting from $\rho_{0}$, along with the cone that they span, and the convex set of deterministic states. 
Definition 65. An element $\sigma$ of $\llbracket \mathrm{A}_{G} \rrbracket_{\rho_{0} Q \mathbb{R}}^{(B)}$ is a quasi-local state if there exists $\sigma_{n R_{n} \rho_{0}}$ in the class $\sigma$, and $n_{0} \in \mathbb{N}$, such that, for $n \geq n_{0}, \sigma_{n R_{n} \rho_{0}} \in$ $\llbracket \mathrm{A}_{G} \rrbracket_{\rho_{0} L}^{(B)}$. The set of quasi-local states in $B$ upon $\rho_{0}$ is denoted by $\llbracket \mathrm{A}_{G} \rrbracket_{\rho_{0} Q}^{(B)}$.

A quasi-local state $\sigma$ in $\llbracket \mathrm{A}_{G} \rrbracket_{\rho_{0} Q}^{(B)}$ is deterministic, if there exists a sequence $\sigma_{n R_{n}}$ in the class of $\sigma$ and $n_{0} \in \mathbb{N}$ such that, for $n \geq n_{0}, \sigma_{n R_{n}} \in$ $\llbracket \mathrm{A}_{G} \rrbracket_{\rho_{0} L 1}^{(B)}$. The set of deterministic quasi-local states is denoted by $\llbracket \mathrm{A}_{G} \rrbracket_{\rho_{0} Q 1}^{(B)}$.

We denote the subset of $\llbracket \mathrm{A}_{G} \rrbracket_{\rho_{0} Q \mathbb{R}}^{(B)}$ of elements $\sigma=\lambda \rho$, for $\lambda \geq 0$ and $\rho \in \llbracket \mathrm{A}_{G} \rrbracket_{\rho_{0} Q}^{(B)}$, as $\llbracket \mathrm{A}_{G} \rrbracket_{\rho_{0} Q+}^{(B)}$. We will equivalently write $\sigma \succeq 0$ for $\sigma \in \llbracket \mathrm{A}_{G} \rrbracket_{\rho_{0} Q+}^{(B)}$

Analogously to the case of effects, the elements of $\llbracket \mathrm{A}_{G} \rrbracket_{\rho_{0} Q \mathbb{R}}^{(B)}$ will be denoted by $\rho=$ $\left[\rho_{n R_{n} \rho_{0}}\right]$, with the convention that for a constant Cauchy sequence $\rho_{n R_{n} \rho_{0}}=\rho_{R \rho_{0}}$ we will set $\rho_{R \rho_{0}}:=\left[\rho_{n R_{n} \rho_{0}}\right]$. We define $\|\rho\|_{B, \rho_{0}}:=$ $\lim _{n \rightarrow \infty}\left\|\rho_{n R_{n} \rho_{0}}\right\|_{B, \rho_{0}}$. One can straightforwardly check that the space $\llbracket \mathrm{A}_{G} \rrbracket_{\rho_{0} Q \mathbb{R}}^{(B)}$ of quasi-local states in $B$ upon $\rho_{0}$ is a Banach space, the sets $\llbracket \mathrm{A}_{G} \rrbracket_{\rho_{0} Q}^{(B)}$ and $\llbracket \mathrm{A}_{G} \rrbracket_{\rho_{0} Q 1}^{(B)}$ are convex subsets, and $\llbracket \mathrm{A}_{G} \rrbracket_{\rho_{0} Q+}^{(B)}$ is a convex cone. The space $\llbracket \mathrm{A}_{G} \rrbracket_{\rho_{0} L \mathbb{R}}^{(B)}$ can be identified with the dense submanifold containing constant sequences $\sigma_{S \rho_{0}}$.

For every partition $B$ and every reference local state $\rho_{0}$, the Banach space $\llbracket \mathrm{A}_{G} \rrbracket_{\rho_{0} Q \mathbb{R}}^{(B)}$ is separable. Indeed, one can choose a basis in $\llbracket \mathrm{A}_{S_{n}} \rrbracket_{\mathbb{R}}$ for each region $S_{n}:=\bigcup_{j=1}^{n} B_{j}$ in the increasing sequence $\left\{S_{n}\right\}_{n \in \mathbb{N}}$ of regions of $B$. Now, for every $\rho \in \llbracket \mathrm{A}_{G} \rrbracket_{\rho_{0} Q \mathbb{R}}^{(B)}$, and for every $n \in \mathbb{N}$, given the class $\rho_{n R_{n} \rho_{0}}$ defining $\rho$, one has $R_{n} \subseteq S_{m}$ for sufficiently large $m$. Thus, linear combinations of the countable collection of bases for $S_{m}$ are dense in $\llbracket \mathrm{A}_{G} \rrbracket_{\rho_{0} Q \mathbb{R}}^{(B)}$.

We now show that actually, for every pair $\left(B, \rho_{0}\right)$, the space $\llbracket \mathrm{A}_{G} \rrbracket_{\rho_{0} Q \mathbb{R}}^{(B)}$ is a subspace of $\llbracket \mathrm{A}_{G} \rrbracket_{\mathbb{R}}$, and $\|\cdot\|_{*} \equiv\|\cdot\|_{B, \rho_{0}}$ on $\llbracket \mathrm{A}_{G} \rrbracket_{\rho_{0} Q \mathbb{R}}^{(B)}$. First of all, we extend the pairing of $\llbracket \overline{\mathrm{A}}_{G} \rrbracket_{Q \mathbb{R}}$ and $\llbracket \mathrm{A}_{G} \rrbracket_{\rho_{0} L \mathbb{R}}^{(B)}$ to $\llbracket \overline{\mathrm{A}}_{G} \rrbracket_{Q \mathbb{R}}$ and $\llbracket \mathrm{A}_{G} \rrbracket_{\rho_{0} Q \mathbb{R}}^{(B)}$.

Definition 66. Let $a=\left[a_{n R_{n}}\right] \in \llbracket \overline{\mathrm{A}}_{G} \rrbracket_{Q \mathbb{R}}$ and $\sigma=\left[\sigma_{n S_{n} \rho_{0}}\right] \in \llbracket \mathrm{A}_{G} \rrbracket_{\rho_{0} Q \mathbb{R}}^{(B)}$. Then we define

$$
(a \mid \sigma):=\lim _{n \rightarrow \infty}\left(a \mid \sigma_{n S_{m} \rho_{0}}\right) .
$$

The above definition is well-posed, as shown by the following lemma, whose proof is a straightforward consequence of lemma 78.

Lemma 80. Let $a \in \llbracket \overline{\mathrm{A}}_{G} \rrbracket_{Q \mathbb{R}}$, and $\sigma=$ $\left[\sigma_{n S_{n} \rho_{0}}\right]=\left[\sigma_{n S_{n}^{\prime} \rho_{0}}^{\prime}\right] \in \llbracket \mathrm{A}_{G} \rrbracket_{\rho_{0} Q \mathbb{R}}^{(B)}$. Then

$$
\lim _{n \rightarrow \infty}\left(a \mid \sigma_{m S_{m} \rho_{0}}\right)=\lim _{n \rightarrow \infty}\left(a \mid \sigma_{m S_{m}^{\prime} \rho_{0}}^{\prime}\right) .
$$

Finally, we use the definition of sup-norm of effects to prove that vectors in the spaces $\llbracket \mathrm{A}_{G} \rrbracket_{\rho_{0} Q \mathbb{R}}^{(B)}$ are bounded linear functionals on $\llbracket \overline{\mathrm{A}}_{G} \rrbracket_{Q \mathbb{R}}$. Also in this case the proof follows straightforwardly form lemma 78 .

Lemma 81. Let $a \in \llbracket \overline{\mathrm{A}}_{G} \rrbracket_{Q \mathbb{R}}$ and $\rho \in \llbracket \mathrm{A}_{G} \rrbracket_{\rho_{0} Q \mathbb{R}}^{(B)}$. Then one has

$$
|(a \mid \rho)| \leq\|a\|_{\text {sup }}\|\rho\|_{B, \rho_{0}} .
$$

Lemma 82. The spaces $\llbracket \mathrm{A}_{G} \rrbracket_{\rho_{0} Q \mathbb{R}}^{(B)}$ are closed subspaces of the extended space of states $\llbracket \mathrm{A}_{G} \rrbracket_{\mathbb{R}}$. On $\llbracket \mathrm{A}_{G} \rrbracket_{\rho_{0} Q \mathbb{R}}^{(B)}$ it holds that $\|\cdot\|_{B, \rho_{0}} \equiv\|\cdot\|_{*}$.

Proof. We only need to prove that $\|\cdot\|_{*} \equiv\|\cdot\|_{Q}$, since we already proved that $\llbracket \mathrm{A}_{G} \rrbracket_{\rho_{0} L \mathbb{R}}^{(B)}$ is a linear manifold in the space of bounded linear functionals on $\llbracket \overline{\mathrm{A}}_{G} \rrbracket_{Q \mathbb{R}}$. First of all, by eq. (134) one clearly has $\|\rho\|_{*} \leq\|\rho\|_{B, \rho_{0}}$ for all $\rho \in$ $\llbracket \mathrm{A}_{G} \rrbracket_{Q \mathbb{R}}$. For the converse, one can use the same technique as for lemma 16, to prove that $\|\rho\|_{B, \rho_{0}} \leq\|\rho\|_{*}$ for the case of a local state $\rho \in \llbracket \mathrm{A}_{G} \rrbracket_{\rho_{0} L \mathbb{R}}^{(B)}$. Thus $\|\rho\|_{*}=\|\rho\|_{B, \rho_{0}}$ for local states $\rho \in \llbracket \mathrm{A}_{G} \rrbracket_{\rho_{0} L \mathbb{R}}^{(B)}$. It is then straightforward to conclude that equality holds for every quasi local state $\rho \in \llbracket \mathrm{A}_{G} \rrbracket_{\rho_{0} Q \mathbb{R}}^{(B)}$.

Notice that two partitions $B$ and $B^{\prime}$ along with two local reference states $\rho_{0}$ and $\rho_{0}^{\prime}$ might define the same space $\llbracket \mathrm{A}_{G} \rrbracket_{\rho_{0} Q \mathbb{R}}^{(B)} \equiv \llbracket \mathrm{A}_{G} \rrbracket_{\rho_{0}^{\prime} Q \mathbb{R}}^{\left(B^{\prime}\right)}$. This is the case if for every $\sigma \in \llbracket \mathrm{A}_{G} \rrbracket_{\rho_{0} Q \mathbb{R}}^{(B)}$ there exists a state $\sigma^{\prime} \in \llbracket \mathrm{A}_{G} \rrbracket_{\rho_{0}^{\prime} Q \mathbb{R}}^{\left(B^{\prime}\right)}$ such that, $\sigma=\sigma^{\prime}$, as functionals on $\llbracket \overline{\mathrm{A}}_{G} \rrbracket Q \mathbb{R}$. We then provide the following definition.

Definition 67. We say that the two reference local states $\left(B, \rho_{0}\right)$ and $\left(B^{\prime}, \rho_{0}^{\prime}\right)$ are compatible, and denote this relation by the symbol $\left(B, \rho_{0}\right) \sim$ $\left(B^{\prime}, \rho_{0}^{\prime}\right)$, if for all $\sigma \in \llbracket \mathrm{A}_{G} \rrbracket_{\rho_{0} Q \mathbb{R}}^{(B)}$ there exists a state $\sigma^{\prime} \in \llbracket \mathrm{A}_{G} \rrbracket_{\rho_{0}^{\prime} Q \mathbb{R}}^{\left(B^{\prime}\right)}$ such that, for every $a \in$ $\llbracket \overline{\mathrm{A}}_{G} \rrbracket_{Q \mathbb{R}}$, one has

$$
(a \mid \sigma)=\left(a \mid \sigma^{\prime}\right) .
$$


The equivalence classes of pairs $\left(B, \rho_{0}\right)$ under the above relation will be denoted by $\left[\left(B, \rho_{0}\right)\right]$.

The norm $\|\cdot\|_{B, \rho_{0}}$ can be extended to the space of finite linear combinations of generalised quasilocal states.

Definition 68. Let $\sigma_{i} \in \llbracket \mathrm{A}_{G} \rrbracket_{\rho_{0}^{(i)} Q \mathbb{R}}^{\left(B^{(i)}\right)}$, for $i=$ $1, \ldots, k$, with $\left[\left(B^{(i)}, \rho_{0}^{(i)}\right)\right] \neq\left[\left(B^{(j)}, \rho_{0}^{(j)}\right)\right]$. Then we define

$$
\left\|\sum_{i=1}^{k} a_{i} \sigma_{i}\right\|_{Q}:=\sum_{i=1}^{k}\left|a_{i}\right|\left\|\sigma_{i}\right\|_{B^{(i)}, \rho_{0}^{(i)}} .
$$

The space of finite sums as from definition 68 is a real vector subspace of $\llbracket \mathrm{A}_{G} \rrbracket_{\mathbb{R}}$. Moreover, as a real vector space, it is equipped with the norm $\|\cdot\|_{Q}$. We can define the space of Cauchy sequences in this space, and complete it by the usual procedure. This leads us to the following definition of the space of quasi-local states.

Definition 69. The space of generalised quasilocal states of $\mathrm{A}_{G}$, denoted as $\llbracket \mathrm{A}_{G} \rrbracket_{Q \mathbb{R}}$, is

$$
\llbracket \mathrm{A}_{G} \rrbracket_{Q \mathbb{R}}:=\bigoplus_{\left[B, \rho_{0}\right]} \llbracket \mathrm{A}_{G} \rrbracket_{\rho_{0} Q \mathbb{R}}^{(B)}
$$

where the direct sum denotes closure of the space of finite linear combinations in the norm $\|\cdot\|_{Q}$.

The norm introduced above allows us to prove that $\llbracket \mathrm{A}_{G} \rrbracket_{Q \mathbb{R}}$ is a space of bounded linear functionals on $\llbracket \overline{\mathrm{A}}_{G} \rrbracket_{Q \mathbb{R}}$, namely a subspace of $\llbracket \mathrm{A}_{G} \rrbracket_{\mathbb{R}}$.

Lemma 83. Every quasi-local state $\rho \in \llbracket \mathrm{A}_{G} \rrbracket_{Q \mathbb{R}}$ is a continuous functional on the space of quasilocal effects $a \in \llbracket \overline{\mathrm{A}}_{G} \rrbracket_{Q \mathbb{R}}$, and

$$
|(a \mid \rho)| \leq\|a\|_{\text {sup }}\|\rho\|_{Q}
$$

Proof. Let $\rho \in \llbracket \mathrm{A}_{G} \rrbracket_{\mathbb{R}}$ be a finite sum of the form

$$
\rho=\sum_{i=1}^{k} a_{i} \sigma_{i}, \quad \sigma_{i} \in \llbracket \mathrm{A}_{G} \rrbracket_{\rho_{0} Q \mathbb{R}}^{\left(B^{(i)}\right)} .
$$

Then

$$
(a \mid \rho):=\sum_{i=1}^{k} a_{i}\left(a \mid \sigma_{i}\right) .
$$

One can easily realise that $|(a \mid \rho)| \leq\|a\|_{\text {sup }}\|\rho\|_{Q}$. Finally, by the above inequality, also the limit of a Cauchy sequence in $\llbracket \mathrm{A}_{G} \rrbracket_{Q \mathbb{R}}$ is a continuous linear functional on $\llbracket \overline{\mathrm{A}}_{G} \rrbracket_{Q \mathbb{R}}$, and satisfies

$$
|(a \mid \rho)| \leq\|a\|_{\text {sup }}\|\rho\|_{Q} .
$$

We can now prove the following important result.

Lemma 84. The space $\llbracket \mathrm{A}_{G} \rrbracket_{Q \mathbb{R}}$ is a closed subspace of the extended space of states $\llbracket \mathrm{A}_{G} \rrbracket_{\mathbb{R}}$. On $\llbracket \mathrm{A}_{G} \rrbracket_{Q \mathbb{R}}$ it holds that $\|\cdot\|_{Q} \equiv\|\cdot\|_{*}$.

Proof. We only need to prove that $\|\cdot\|_{*} \equiv\|\cdot\|_{Q}$ on finite sums of the form of eq. (136), since we already proved that $\llbracket \mathrm{A}_{G} \rrbracket_{Q \mathbb{R}}$ is obtained closing a linear manifold in the space of bounded linear functionals on $\llbracket \overline{\mathrm{A}}_{G} \rrbracket_{Q \mathbb{R}}$. The proof follows exactly the same argument as for lemma 82, using eq. (135) instead of eq. (134).

Notice that all local states allow us to define states of arbitrary finite regions $S \subseteq G$, by Eq. (122). We will now extend the definition of local state for all quasi-local states in a fixed sector $\llbracket \mathrm{A} \rrbracket_{\rho_{0} Q}^{(B)}$, as follows. First, if $\rho$ is quasi local, then $\left\|\rho_{n}-\rho_{m}\right\|_{B, \rho_{0}} \leq \varepsilon$, and $\left\|\left.\left(\rho_{n}-\rho_{m}\right)\right|_{S}\right\|_{\text {op }} \leq$ $\left\|\rho_{n}-\rho_{m}\right\|_{B, \rho_{0}} \leq \varepsilon$. Thus, being the set of states $\llbracket \mathrm{A}_{S} \rrbracket$ compact, $\rho_{S}:=\left.\lim _{n \rightarrow \infty} \rho_{n}\right|_{S} \in \llbracket \mathrm{A}_{S} \rrbracket$.

The same is true for finte linear combinations, as well as limits of Cauchy sequences thereof. Indeed, given a finite combination of states $\rho=$ $\sum_{i} a_{i} \rho_{i}$, one has

$$
\rho_{\mid S}:=\sum_{i} a_{i} \rho_{i \mid S} \in \llbracket \mathrm{A}_{S} \rrbracket \mathbb{R} .
$$

Finally, if $\tau: \mathbb{N} \rightarrow \llbracket \mathrm{A} \rrbracket_{Q \mathbb{R}}$, and $\left\|\tau_{n}-\tau_{m}\right\|_{Q} \leq \varepsilon$, then clearly $\left\|\left(\tau_{n}-\tau_{m}\right)_{\left.\right|_{S}}\right\|_{\mathrm{op}} \leq \varepsilon$, and thus

$$
\tau_{\mid S}:=\lim _{n \rightarrow \infty}\left(\tau_{n}\right)_{\mid S} \in \llbracket \mathrm{A}_{S} \rrbracket_{\mathbb{R}} .
$$

In view of the above considerations, we can now define the set of states in $\llbracket \mathrm{A}_{G} \rrbracket_{Q \mathbb{R}}$.

Definition 70. The set $\llbracket \mathrm{A}_{G} \rrbracket_{Q}$ of quasi-local states is the (convex) set of generalised states $\rho$ for which, for every finite region $S \in \mathrm{R}^{(G)}$, the restriction $\rho_{\mid S}$ of $\rho$ to $S$ is a state in $\llbracket \mathrm{A}_{S} \rrbracket$. The positive cone $\llbracket \mathrm{A}_{G} \rrbracket_{Q+}$ of $\mathrm{A}_{G}$ is

$$
\begin{aligned}
& \llbracket \mathrm{A}_{G} \rrbracket_{Q+} \\
& :=\left\{\sigma \in \llbracket \mathrm{A}_{G} \rrbracket_{Q \mathbb{R}} \mid \exists \lambda \geq 0, \rho \in \llbracket \mathrm{A}_{G} \rrbracket_{Q}: \sigma=\lambda \rho\right\} .
\end{aligned}
$$

The set of deterministic quasi-local states of $\mathrm{A}_{G}$, denoted as $\llbracket \mathrm{A}_{G} \rrbracket_{Q 1}$, is

$$
\llbracket \mathrm{A}_{G} \rrbracket_{Q 1}:=\left\{\rho \in \llbracket \mathrm{A}_{G} \rrbracket_{Q} \mid\left(e_{G} \mid \rho\right)=1\right\} .
$$

Lemma 85. Definition 70 is compatible with definition 16 
Proof. We need to prove that the definition of restriction to the finite region $S$ provided in Eq. (33) is equivalent to that of Eq. (139). We start from the case of local states. In this case, since effects are separating for states of finite dimensional systems, one has $\left(a_{S} \mid \rho_{T}\right)=\left(a_{S} \otimes\right.$ $e_{T \backslash S} \mid \rho_{\mid T} \otimes \rho_{0 \mid S \backslash T}$, and thus $\rho_{\mid S}=\rho_{\mid(T \cap S)} \otimes \rho_{0 \mid S \backslash T}$, which is precisely the same as in Eq. (122). The statement in the case of a general state $\rho \in$ $\llbracket \mathrm{A}_{G} \rrbracket_{Q}$ is then straightforwardly proved, reminding that $\left|\left(a_{S} \mid \rho-\rho_{n}\right)\right| \leq\left\|a_{S}\right\|_{\text {sup }}\left\|\rho-\rho_{n}\right\|_{Q}$.

Corollary 19. One has the following inclusions

$$
\llbracket \mathrm{A}_{G} \rrbracket_{Q} \subseteq \llbracket \mathrm{A}_{G} \rrbracket, \quad \llbracket \mathrm{A}_{G} \rrbracket_{Q 1} \subseteq \llbracket \mathrm{A}_{G} \rrbracket_{1} .
$$

\section{Proof of identity 94}

In order to keep the notation lighter, we do the calculation for the case of $\mathrm{C} \cong \mathrm{I}$. The proof can then be adapted to the general case by suitably padding all transformations with $\mathscr{I}_{\mathrm{C}}$ and extending the region $\tilde{S}$ to $\tilde{S} \mathrm{C}$.

$$
\begin{aligned}
& \left(\mathscr{V} \mathscr{A}^{-1} \otimes \mathscr{I}_{H_{1}}\right)^{\dagger} a_{\tilde{S}}=\mathscr{W}^{-1 \dagger}\left(\mathscr{A} \otimes \mathscr{I}_{H_{1}}\right)^{\dagger} \mathscr{W}^{\dagger} a_{\tilde{S}} \\
& =\mathscr{W}^{-1 \dagger}\left(\mathscr{A} \otimes \mathscr{I}_{H_{1}}\right)^{\dagger} \mathscr{S}_{N_{S}^{+*} \cup N_{S}^{+}}^{+} \mathscr{S}_{N_{S}^{+*} \cup S}^{\prime \dagger} a_{\tilde{S}} \\
& =\mathscr{S}_{P_{S}^{+*} \cup N_{S}^{+*} \cup R^{\prime}}^{\mathscr{S}_{N_{S}^{+}}^{\dagger} \cup N_{S}^{+*} \cup R}\left(\mathscr{A} \otimes \mathscr{I}_{H_{1}}\right)^{\dagger} \\
& \times \mathscr{S}_{N_{S}^{+*} \cup N_{S}^{+}}^{\dagger} \mathscr{S}_{N_{S}^{+*} \cup S}^{\prime \dagger} a_{\tilde{S}} \\
& =\mathscr{S}_{P_{S}^{\prime \dagger} \cup N_{S}^{+*} \cup R^{S_{N}} \mathscr{N}_{S}^{+} \cup N_{S}^{+*} \cup R}\left(\mathscr{A} \otimes \mathscr{I}_{H_{1}}\right)^{\dagger} \\
& \times \mathscr{S}_{N_{S}^{+} \cup N_{S}^{+*} \cup R}^{\dagger} \mathscr{S}_{P_{S}^{+*} \cup N_{S}^{+*} \cup R}^{a_{\tilde{S}}} \\
& =\mathscr{S}_{P_{S}^{+*} \cup N_{S}^{+*} \cup R} \mathscr{S}_{R}^{\dagger}\left(\mathscr{A} \otimes \mathscr{I}_{H_{1}}\right)^{\dagger} \mathscr{S}_{R}^{\dagger} \mathscr{S}_{P_{S}^{+*} \cup N_{S}^{+*} \cup R} a_{\tilde{S}} \\
& =\mathscr{S}_{P_{S}^{+*} \cup N_{S}^{+*} \cup R}^{\prime \dagger}\left(\mathscr{I}_{H_{0}} \otimes \mathscr{A}\right)^{\dagger} \mathscr{S}_{P_{S}^{+*} \cup N_{S}^{+*} \cup R}^{a_{\tilde{S}}} \\
& =\mathscr{S}_{R}^{\prime \dagger}\left(\mathscr{I}_{H_{0}} \otimes \mathscr{A}\right)^{\dagger} \mathscr{S}_{R}^{\prime \dagger}\left[a_{\tilde{S}}\right] \\
& =\mathscr{S}_{R}^{\prime \dagger} \mathscr{S}_{R}^{\dagger}\left(\mathscr{A} \otimes \mathscr{I}_{H_{0}}\right)^{\dagger} \mathscr{S}_{R}^{\dagger} \mathscr{S}_{R}^{\prime \dagger}\left[a_{\tilde{S}}\right] \text {, }
\end{aligned}
$$

where in the third identity we used Eq. (91), observing that $\left(\mathscr{A} \otimes \mathscr{I}_{H_{1}}\right)^{\dagger} \mathscr{S}_{N_{S}^{+*} \cup N_{S}^{+}}^{\dagger} \mathscr{S}_{N_{S}^{+*} \cup S}^{\prime \dagger}\left[a_{\tilde{S}}\right] \in$ $\llbracket \overline{\mathrm{A}}_{\left(N_{S}^{+*} \cup R, 0\right.}^{(G)} \cup\left(N_{S}^{+}, 1\right) \rrbracket_{Q \mathbb{R}}$. In the fourth identity we used the fact that $a_{\tilde{S}}$ can be padded with $e_{X}$ to obtain an effect $\tilde{a}_{X}$ on the region $\tilde{S} \cup X$, and then $\mathscr{W}^{\dagger} \tilde{a}_{X}$ is an effect equivalent to $\mathscr{W}^{\dagger} a_{S}$. In the fifth identity we used the fact that $\mathscr{S}_{X}$ commutes with $\mathscr{Y}_{Y} \otimes \mathscr{I}_{H_{1}}$ for $Y \cap X=\emptyset$, and in the seventh identity we used the fact that $\mathscr{S}_{X}^{\prime}$ commutes with $\mathscr{I}_{H_{0}} \otimes \mathscr{Y}_{Y}$ for $Y \cap X=\emptyset$.

\section{Homogeneity and causal influence}

This section is devoted to the proof that, for $\pi \in$ $\Pi_{\mathscr{V}}$

$$
\pi(g) \longmapsto \pi\left(g^{\prime}\right) \Leftrightarrow g \longmapsto g^{\prime}
$$

By definition, $f \longmapsto f^{\prime}$ if there exists $R \ni f$ and $\exists \mathscr{F} \in \llbracket \mathrm{A}_{f}^{(G)} \mathrm{C} \rightarrow \mathrm{A}_{f}^{(G)} \mathrm{C} \rrbracket_{Q \mathbb{R}}$,

$\left(\mathscr{V} \otimes \mathscr{I}_{\mathrm{C}}\right) \mathscr{F}\left(\mathscr{V}^{-1} \otimes \mathscr{I}_{\mathrm{C}}\right) \notin \llbracket \mathrm{A}_{\bar{f}^{\prime}}^{(G)} \mathrm{C} \rightarrow \mathrm{A}_{\bar{f}^{\prime}}^{(G)} \mathrm{C} \rrbracket_{Q \mathbb{R}}$.

Let now $f=\pi(g)$ for some $\pi \in \Pi_{\mathscr{V}}$. Now, by homogeneity, and in particular by Eq. (104), one has that $\mathscr{F}^{\prime} \in \llbracket \mathrm{A}_{\pi(g)}^{(G)} \mathrm{C} \rightarrow \mathrm{A}_{\pi(g)}^{(G)} \mathrm{C} \rrbracket$ if and only if there exists $\mathscr{F} \in \llbracket \mathrm{A}_{g}^{(G)} \mathrm{C} \rightarrow \mathrm{A}_{g}^{(G)} \mathrm{C} \rrbracket$ such that $\mathscr{F}^{\prime}=\left(\mathscr{T}_{\pi} \otimes \mathscr{I}_{\mathrm{C}}\right) \mathscr{F}\left(\mathscr{T}_{\pi}^{-1} \otimes \mathscr{I}_{\mathrm{C}}\right)$. Then one has

$$
\begin{aligned}
\left(\mathscr{V} \otimes \mathscr{I}_{\mathrm{C}}\right) & \mathscr{F}^{\prime}\left(\mathscr{V}^{-1} \otimes \mathscr{I}_{\mathrm{C}}\right) \\
& =\left(\mathscr{V} \mathscr{T}_{\pi} \otimes \mathscr{I}_{\mathrm{C}}\right) \mathscr{F}\left(\mathscr{T}_{\pi}^{-1} \mathscr{V}^{-1} \otimes \mathscr{I}_{\mathrm{C}}\right) \\
& =\left(\mathscr{T}_{\pi} \mathscr{V} \otimes \mathscr{I}_{\mathrm{C}}\right) \mathscr{F}\left(\mathscr{V}^{-1} \mathscr{T}_{\pi}^{-1} \otimes \mathscr{I}_{\mathrm{C}}\right) .
\end{aligned}
$$

As a consequence, again by eq. (104), we have that $\left(\mathscr{V} \otimes \mathscr{I}_{\mathrm{C}}\right) \mathscr{F}^{\prime}\left(\mathscr{V}^{-1} \otimes \mathscr{I}_{\mathrm{C}}\right) \in \llbracket \mathrm{A}_{\bar{f}^{\prime}}^{(G)} \mathrm{C} \rightarrow$ $\mathrm{A}_{\bar{f}^{\prime}}^{(G)} \mathrm{C} \rrbracket$, for $f^{\prime}=\pi\left(g^{\prime}\right)$, if and only if $(\mathscr{V} \otimes$

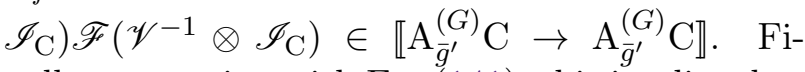
nally, comparing with Eq. (141), this implies that $\pi(g) \longmapsto \pi\left(g^{\prime}\right)$ if and only if $g \longmapsto g^{\prime}$.

\section{E Proof of right and left invertibility of a locally defined GUR}

Here we prove right and left invertibility of $\mathscr{V}$ defined starting from Eq. (109). First, notice that
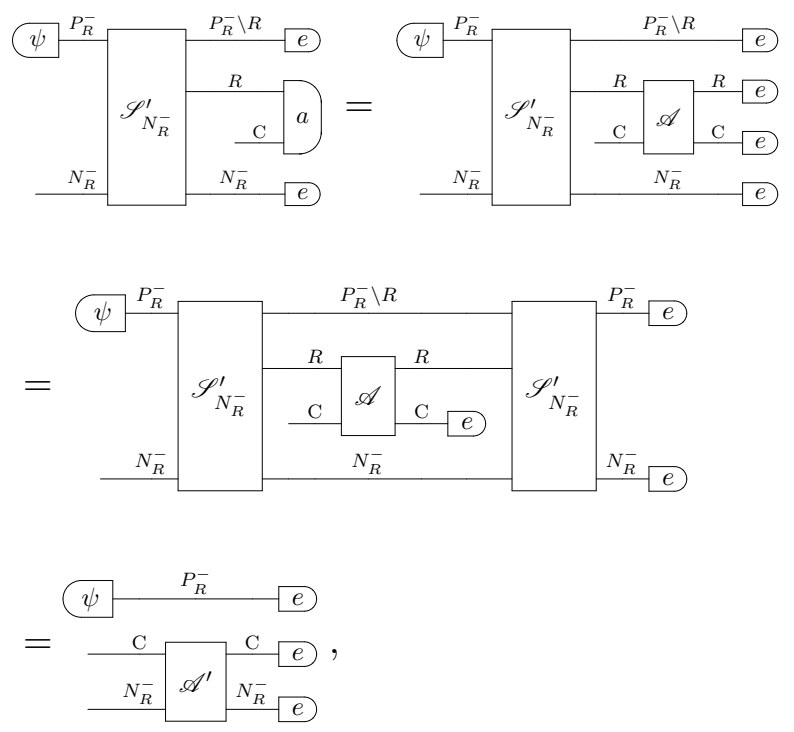
where we used Eq. (94).

Let us now define $\mathscr{W}$ by $\mathscr{W}^{\dagger} b_{S \mathrm{C}}:=\left\{\left(\mathscr{W}_{S}^{\dagger} \otimes\right.\right.$ $\left.\left.\mathscr{I}_{\mathrm{C}}^{\dagger}\right) b\right\}_{N_{S}^{+} \mathrm{C}}$, where

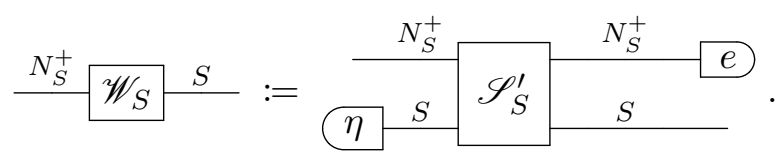

Then one can calculate $\mathscr{W}^{\dagger} \mathscr{V}^{\dagger} a_{R \mathrm{C}}$ by the following diagram

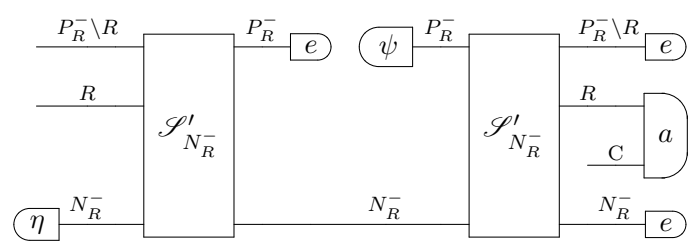

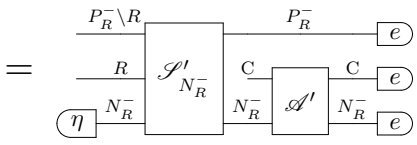

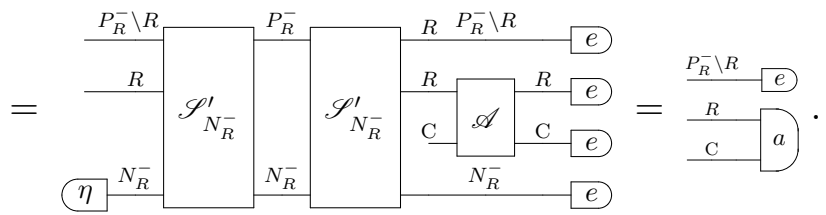

Finally, we observe that $\left[\left(a \otimes e_{P_{R}^{-} \backslash R}\right)_{P_{R}^{-} \mathrm{C}}\right]=$ $\left[a_{R \mathrm{C}}\right]$, then $\mathscr{W}^{\dagger} \mathscr{V}^{\dagger}=\mathscr{I}_{G}^{\dagger}$. Similarly, one can calculate $\mathscr{V}^{\dagger} \mathscr{W}^{\dagger} b_{S \mathrm{C}}$. First we observe that $\left[b_{S \mathrm{C}}\right]=$ $\left[\left(b \otimes e_{P_{S}^{+} \backslash S}\right)_{P_{S}^{+} \mathrm{C}}\right]$. Then $\mathscr{W}^{\dagger} b_{S \mathrm{C}}=\left\{\left(\mathscr{W}_{P_{S}^{+}}^{\dagger}(b \otimes\right.\right.$ $\left.\left.e_{N_{P_{S}^{+}}^{+} \backslash N_{S}^{+}}\right)\right\}_{N_{P_{S}^{+} \mathrm{C}}^{+}}$, and
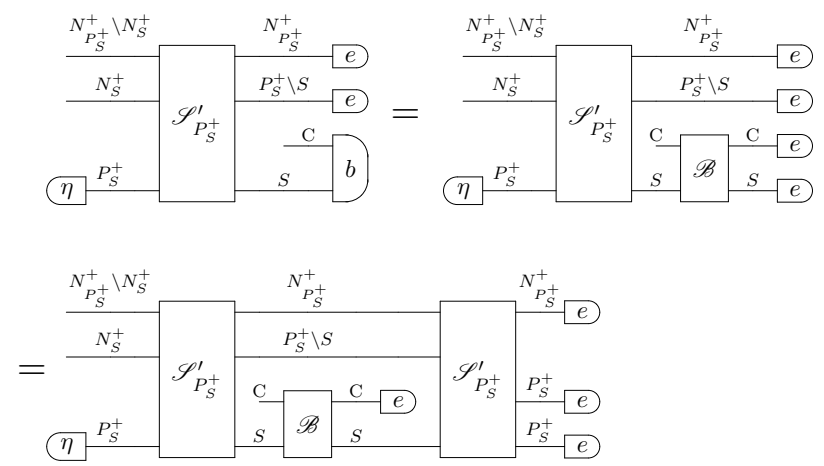

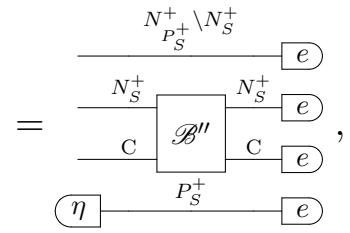

where we used Eq. (97). Now, for $\mathscr{V}^{\dagger} \mathscr{W}^{\dagger}\left[b_{S \mathrm{C}}\right]$ we have
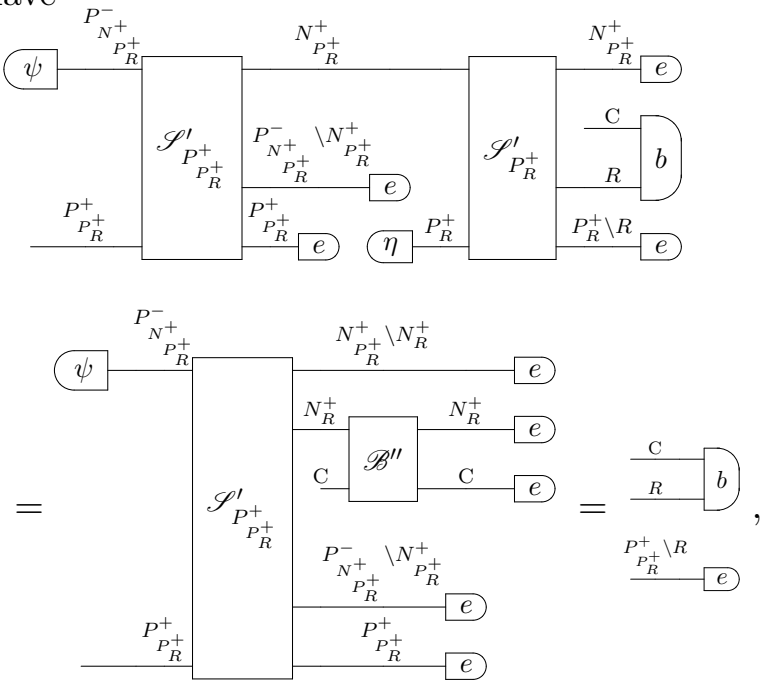

where Eq. (97) was used in the last step. The arguments are straightforwardly generalised to $b \in \llbracket \overline{\mathrm{A}}_{S}^{(G)} \overline{\mathrm{C}} \rrbracket_{Q \mathbb{R}}$.

\section{F Proof of theorem 10}

Proof. In the following, $G_{i}:=(G, i)$ for $i=0,1$. The argument in appendix E shows that

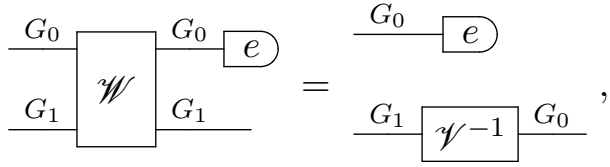

where $\left(G, \mathrm{~A}, \mathscr{V}^{-1 \dagger}\right)$ is a GUR. Moreover, since

$$
\begin{array}{l|l}
G_{0} \\
G_{1}
\end{array} \mathscr{S}_{G}^{G_{1}}=\begin{aligned}
& G_{0} \\
& e
\end{aligned},
$$

one has
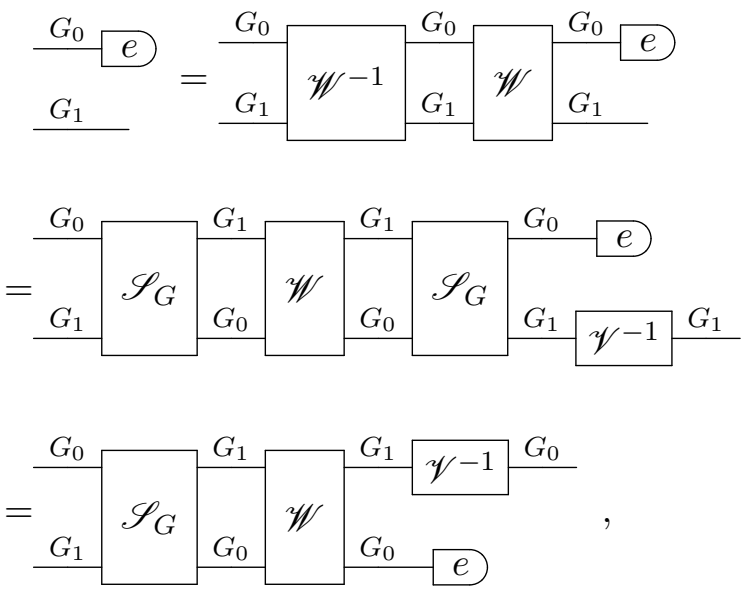

namely

$$
\begin{aligned}
& G_{1} \\
& G_{0} e
\end{aligned}=\begin{aligned}
& G_{1} \\
& G_{0}
\end{aligned} \stackrel{G_{1} \mathscr{V}^{-1} G_{0}}{G_{0}} e
$$


On the other hand, one can write the latter as

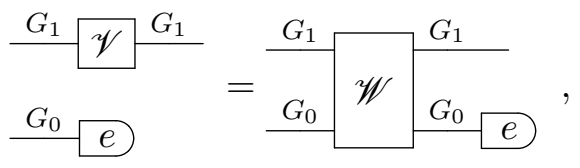

or equivalently

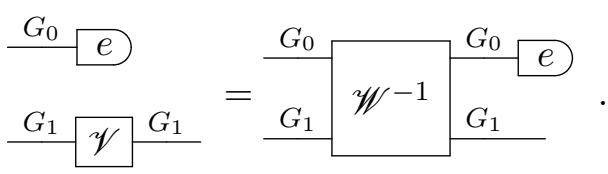

Now, let us consider
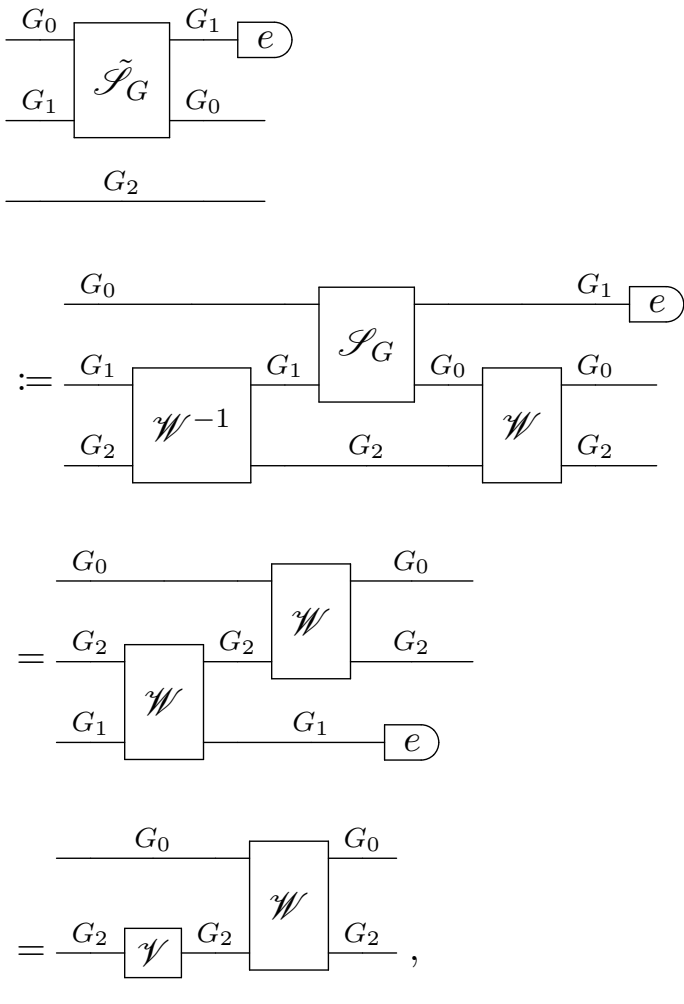

$$
G_{1} e
$$

from which one concludes that

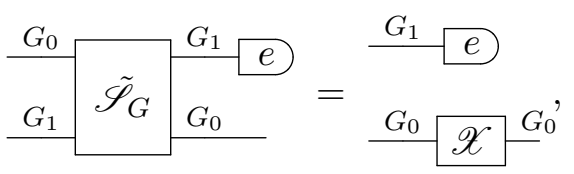

for some $\mathscr{X}$. Thus

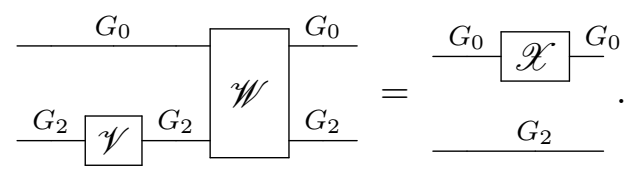

Finally, this implies that $\mathscr{W}=\mathscr{X} \otimes \mathscr{V}^{-1}$, and in particular, by Eq. (144), it must be $\mathscr{X}=\mathscr{V}$, namely $\mathscr{W}=\mathscr{V} \otimes \mathscr{V}^{-1}$. Consequently, we have that
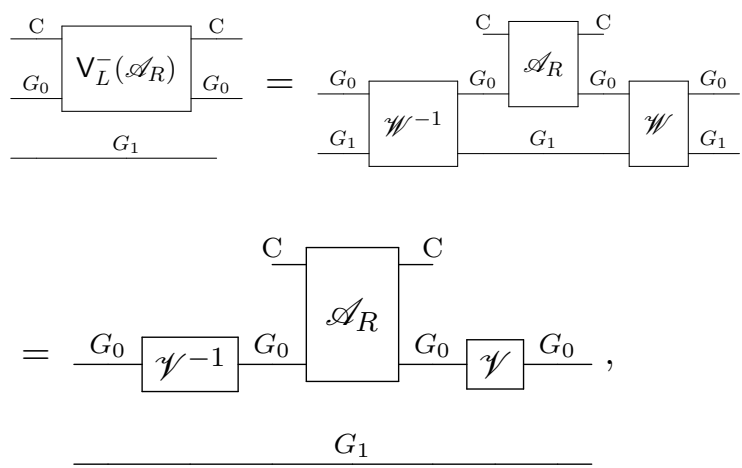

and

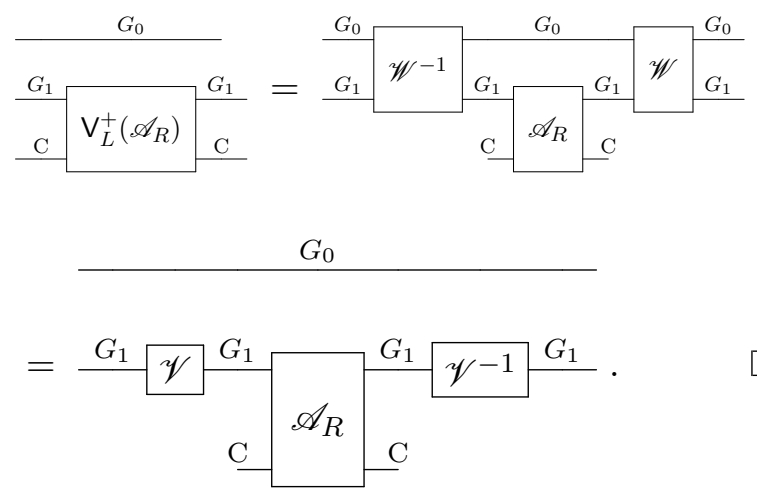

\section{G Proof of lemmas 66 and 67}

We start with the proof of lemma 66.

Proof. (1) Notice that $N_{e_{H}}^{\prime \pm}=\phi\left(S_{ \pm}\right)=\phi\left(N_{e_{G}}^{ \pm}\right)$. Then $\left|N_{e_{H}}^{\prime \pm}\right| \leq\left|N_{e_{G}}^{ \pm}\right|$, with strict inequality if and only if $\phi\left(h_{a}\right)=\phi\left(h_{b}\right)$ for some $h_{a} \neq h_{b} \in N_{e_{G}}^{ \pm}$. However, this is impossible because it would be equivalent to $\phi\left(h_{a} h_{b}^{-1}\right) \in R^{\prime}$, namely $h_{a}=h_{b}$, contrarily to the hypothesis. Thus, $\left|N_{e_{H}}^{ \pm}\right|=$ $\left|N_{e_{G}}^{ \pm}\right|$. Invariance of neighbourhoods under leftmultiplication finally gives the thesis.

(2) Let now $f \in P_{e_{H}}^{\prime \pm}=N_{N_{e_{H}}^{\prime \pm}}^{\prime \mp}$, namely there exists $h \in N_{e_{H}}^{\prime \pm}$ such that $f \in N_{h}^{\prime \mp}$, or equivalently $h \in N_{f}^{\prime \pm}$. Then, $f \in P_{e_{H}}^{\prime \pm}$ iff there exists $h \in N_{e_{H}}^{\prime \pm} \cap N_{f}^{\prime \pm}$. Equivalently, there exists $h \in H$ such that $h=\phi\left(h_{a}\right)$ and $h=f \phi\left(h_{b}\right)$, namely $f=\phi\left(h_{a} h_{b}^{-1}\right)$. Then $P_{e_{H}}^{\prime \pm}=\left\{\phi\left(h_{a} h_{b}^{-1}\right) \mid h_{a}, h_{b} \in\right.$ $\left.N_{e_{G}}^{ \pm}\right\}$. By a similar argument, we have that $P_{e_{G}}^{ \pm}=\left\{h_{a} h_{b}^{-1} \mid h_{a}, h_{b} \in N_{e_{G}}^{ \pm}\right\}$. Consequently, $\left|P_{e_{H}}^{\prime \pm}\right| \leq\left|\left\{h_{a} h_{b}^{-1} \mid h_{a}, h_{b} \in N_{e_{G}^{+}}^{+}\right\}\right|=\left|P_{e_{G}}^{ \pm}\right|$. Now, strict inequality in the last relation would require that $\phi\left(h_{a} h_{b}^{-1}\right)=\phi\left(h_{d} h_{c}^{-1}\right)$, contrarily to the hypothesis. We conclude that $\left|P_{e_{H}}^{\prime \pm}\right|=\left|P_{e_{G}}^{ \pm}\right|$. Also in this case the thesis can be obtained by invariance of neighbourhoods under left-multiplication. 
(3) Let $N_{f_{1}}^{\prime \pm} \cap N_{f_{2}}^{\prime \pm}=\emptyset$. Then clearly for every $g_{i} \in \phi^{-1}\left(f_{i}\right)$ it must be $N_{g_{1}}^{ \pm} \cap N_{g_{2}}^{ \pm}=\emptyset$. Let then now $h \in N_{f_{1}}^{ \pm} \cap N_{f_{2}}^{\prime \pm}$. This implies that $h=f_{1} \phi\left(h_{a}\right)=f_{2} \phi\left(h_{b}\right)$ for $h_{a}, h_{b} \in N_{e_{G}}^{ \pm}$. Consequently, $f_{2}=f_{1} \phi\left(h_{a} h_{b}^{-1}\right)$. Now, either $f_{1}=f_{2}$, and then choosing $g_{1}=g_{2}$ the thesis trivially follows, or $\phi\left(h_{a} h_{b}^{-1}\right) \notin R^{\prime}$, and then $h_{a} h_{b}^{-1} \notin R$. One can then set $g_{2}=g_{1} h_{a} h_{b}^{-1}$, and verify that $g_{2} \in \phi^{-1}\left(f_{2}\right)$, as well as $k=g_{1} h_{a}=g_{2} h_{b} \in$ $N_{g_{1}}^{ \pm} \cap N_{g_{2}}^{ \pm}$. Thus, $h=\phi(k)$, and consequently $N_{f_{1}}^{\prime \pm} \cap N_{f_{2}}^{\prime \pm} \subseteq \phi\left(N_{g_{1}}^{ \pm} \cap N_{g_{2}}^{ \pm}\right)$. This implies that $\left|N_{f_{1}}^{\prime \pm} \cap N_{f_{2}}^{\prime \pm}\right| \leq\left|N_{g_{1}}^{ \pm} \cap N_{g_{2}}^{ \pm}\right|$, with a strict inequality iff $\phi\left(k_{1}\right)=\phi\left(k_{2}\right)$ for $k_{1} \neq k_{2} \in N_{g_{1}}^{ \pm} \cap N_{g_{2}}^{ \pm}$. However, form the proof of item (1) we know that if $k_{1} \neq k_{2} \in N_{g}^{ \pm}$it must be $\phi\left(k_{1}\right) \neq \phi\left(k_{2}\right)$. Thus, $\left|N_{f_{1}}^{\prime \pm} \cap N_{f_{2}}^{\prime \pm}\right|=\left|\phi\left(N_{g_{1}}^{ \pm} \cap N_{g_{2}}^{ \pm}\right)\right|$, and $N_{f_{1}}^{\prime \pm} \cap N_{f_{2}}^{\prime \pm}=$ $\phi\left(N_{g_{1}}^{ \pm} \cap N_{g_{2}}^{ \pm}\right)$.

(4) Let $h \in P_{f_{2}}^{\prime \mp} \cap N_{f_{1}}^{\prime \pm}$. Then $h=f_{2} \phi\left(h_{c}^{-1} h_{b}\right)=$ $f_{1} \phi\left(h_{a}\right)$, with $h_{a}, h_{b}, h_{c} \in N_{e_{G}}^{ \pm}$. This implies that $f_{2}=f_{1} \phi\left(h_{a} h_{b}^{-1} h_{c}\right)$. We can now set $g_{2}:=$ $g_{1} h_{a} h_{b}^{-1} h_{c}$ with $g_{1} \in \phi^{-1}\left(f_{1}\right)$. In this way, one has $f_{2}=\phi\left(g_{2}\right)$. Let us set $k:=g_{1} h_{a}=$ $g_{2} h_{c}^{-1} h_{b} \in P_{g_{2}}^{\mp} \cap N_{g_{1}}^{ \pm}$. Then, $h=\phi(k)$, and $P_{f_{1}}^{\prime \mp} \cap N_{f_{1}}^{\prime \pm} \subseteq \phi\left(P_{g_{2}}^{\mp} \cap N_{g_{1}}^{ \pm}\right)$. By the same argument as for item (3), the thesis follows.

(5) Let $h \in P_{g_{1}}^{ \pm} \cap N_{g_{2}}^{\mp}$. Then $h=g_{1} h_{a} h_{b}^{-1}, \quad h=$ $g_{2} h_{c}^{-1}$, for some $h_{a}, h_{b}, h_{c} \in N_{e_{G}}^{ \pm}$, and thus $g_{2}=$ $g_{1} h_{a} h_{b}^{-1} h_{c}$. On the other hand, by hypothesis, for every $h_{x} \in N_{e_{G}}^{ \pm}$one has $g_{2} \neq g_{1} h_{x}$. Collecting all the informations, we have $h_{a} h_{b}^{-1} h_{c} h_{x}^{-1} \notin R$, i.e. $\phi\left(h_{a} h_{b}^{-1} h_{c} h_{x}^{-1}\right) \notin R^{\prime}$, for every $h_{x} \in N_{e_{G}}^{ \pm}$. Then one has $\phi\left(g_{2}\right)=\phi\left(g_{1}\right) \phi\left(h_{a} h_{b}^{-1} h_{c}\right)$. By hypothesis, it is also $\phi\left(g_{2}\right)=\phi\left(g_{1}\right) \phi\left(h_{d}\right)$ for some $h_{d} \in N_{e_{G}}^{ \pm}$. This is clearly absurd, thus it must be $P_{g_{1}}^{ \pm} \cap N_{g_{2}}^{+}=\emptyset$.

Now, we provide the proof of lemma 67

Proof. (1) Let $f_{2} \in P_{f_{1}}^{\prime \pm}$, namely $f_{2}=$ $f_{1} \phi\left(h_{a} h_{b}^{-1}\right)$ for $h_{a}, h_{b} \in N_{e_{G}}^{ \pm}$. If $f_{1}=f_{2}$, it is sufficient to choose $g_{1}=g_{2} \in \phi^{-1}\left(f_{1}\right)$. Let now $f_{1} \neq f_{2}$. Then, let $g_{1} \in \phi^{-1}\left(f_{1}\right)$, and $g_{2}:=g_{1} h_{a} h_{b}^{-1}$. By construction, $\phi\left(g_{2}\right)=f_{2}$ and $g_{2} \in P_{g_{1}}^{ \pm}$. Now, let $h \in P_{f_{1}}^{\prime \pm} \cap P_{f_{2}}^{\prime \pm}$, namely $h=$ $f_{1} \phi\left(h_{f} h_{e}^{-1}\right)=f_{2} \phi\left(h_{c} h_{d}^{-1}\right)=f_{1} \phi\left(h_{a} h_{b}^{-1} h_{c} h_{d}^{-1}\right)$. Then we have $\phi\left(h_{a} h_{b}^{-1} h_{c} h_{d}^{-1} h_{e} h_{f}^{-1}\right) \in R^{\prime}$, and necessarily $h_{a} h_{b}^{-1} h_{c} h_{d}^{-1} h_{e} h_{f}^{-1} \in R$. This implies that $g_{1} h_{f} h_{e}^{-1}=g_{1} h_{a} h_{b}^{-1} h_{c} h_{d}^{-1}=g_{2} h_{c} h_{d}^{-1}$. Now, setting $k:=g_{1} h_{f} h_{e}^{-1}$, we have $k=g_{2} h_{c} h_{d}^{-1} \in$ $P_{g_{1}}^{ \pm} \cap P_{g_{2}}^{ \pm}$, and $h=\phi(k)$. Thus, $P_{f_{1}}^{\prime \pm} \cap P_{f_{2}}^{\prime \pm} \subseteq$
$\phi\left(P_{g_{1}}^{ \pm} \cap P_{g_{2}}^{ \pm}\right)$. Finally, by the same argument as for the proof of item (2) of lemma 66, we get the thesis.

(2) The hypothesis clearly implies that $N_{g_{1}}^{ \pm} \cap$ $N_{g_{2}}^{ \pm}=\emptyset$. Suppose now that $h \in P_{g_{1}}^{ \pm} \cap P_{g_{2}}^{ \pm}$. Then $h=g_{1} h_{a} h_{b}^{-1}=g_{2} h_{d} h_{c}^{-1}$ for $h_{a}, h_{b}, h_{c}, h_{d} \in N_{e_{G}}^{ \pm}$. This implies that $g_{2}=g_{1} h_{a} h_{b}^{-1} h_{c} h_{d}^{-1}$, and consequently $\phi\left(g_{2}\right)=\phi\left(g_{1}\right) \phi\left(h_{a} h_{b}^{-1} h_{c} h_{d}^{-1}\right)$. On the other hand, we proved that for every $h_{e}, h_{f} \in$ $N_{e_{G}}^{ \pm}$one has $g_{2} \neq g_{1} h_{f} h_{e}^{-1}$, while by hypothesis $\phi\left(g_{2}\right)=\phi\left(g_{1}\right) \phi\left(h_{f} h_{e}^{-1}\right)$ for some $h_{e}, h_{f} \in N_{e_{G}}^{ \pm}$. Thus, while $h_{a} h_{b}^{-1} h_{c} h_{d}^{-1} h_{e} h_{f}^{-1} \notin R$, it must be $\phi\left(h_{a} h_{b}^{-1} h_{c} h_{d}^{-1} h_{e} h_{f}^{-1}\right) \notin R^{\prime}$. We reached a contradiction, and thus it must be $P_{g_{1}}^{ \pm} \cap P_{g_{2}}^{ \pm}=\emptyset$.

\section{References}

[1] Lucien Hardy. Disentangling nonlocality and teleportation, 1999. arXiv:quant-ph/ 9906123.

[2] Lucien Hardy. Quantum theory from five reasonable axioms, 2001. arXiv:quant-ph/ 0101012.

[3] Christopher A. Fuchs. Quantum mechanics as quantum information (and only a little more), 2002. arXiv:quant-ph/0205039.

[4] Gilles Brassard. Is information the key? $\mathrm{Na}$ ture Physics, 1(1):2-4, 2005. URL: https: //doi.org/10.1038/nphys134.

[5] Jonathan Barrett. Information processing in generalized probabilistic theories. Phys. Rev. A, 75:032304, Mar 2007. URL: https:// doi.org/10.1103/PhysRevA.75.032304.

[6] Giacomo M. D'Ariano. Probabilistic theories: What is special about quantum mechanics? In Alisa Bokulich and Gregg Jaeger, editors, Philosophy of Quantum Information and Entanglement, chapter 5, pages 85-126. Cambridge University Press., 2010. URL: https://doi.org/10.1017/ CB09780511676550.007.

[7] Giacomo Mauro D'Ariano and Alessandro Tosini. Testing axioms for quantum theory on probabilistic toy-theories. Quantum Information Processing, 9(2):95-141, 2010. URL: https://doi.org/10.1007/ s11128-010-0172-3.

[8] Giulio Chiribella, Giacomo Mauro D'Ariano, and Paolo Perinotti. Probabilistic theories 
with purification. Phys. Rev. A, 81:062348, Jun 2010. URL: https://doi.org/10. 1103/PhysRevA.81.062348.

[9] Giulio Chiribella, Giacomo Mauro D'Ariano, and Paolo Perinotti. Informational derivation of quantum theory. Phys. Rev. A, 84:012311, Jul 2011. URL: https://doi. org/10.1103/PhysRevA . 84.012311.

[10] Lluís Masanes and Markus P Müller. A derivation of quantum theory from physical requirements. New Journal of Physics, 13(6):063001, jun 2011. URL: https://doi. org/10.1088/1367-2630/13/6/063001.

[11] Borivoje Dakić and Časlav Brukner. Quantum theory and beyond: is entanglement special? In H. Halvorson, editor, Deep Beauty: Understanding the Quantum World through Mathematical Innovation, pages 365-392. Cambridge University Press, 2011. URL: https://doi.org/10. 1017/CB09780511976971.011.

[12] Peter Rastall. Locality, Bell's theorem, and quantum mechanics. Foundations of Physics, 15(9):963-972, Sep 1985. URL: https://doi.org/10.1007/BF00739036.

[13] Sandu Popescu and Daniel Rohrlich. Quantum nonlocality as an axiom. Foundations of Physics, 24(3):379-385, Mar 1994. URL: https://doi.org/10.1007/BF02058098.

[14] Robert W. Spekkens. Evidence for the epistemic view of quantum states: A toy theory. Phys. Rev. A, 75:032110, Mar 2007. URL: https://doi.org/10.1103/ PhysRevA.75.032110.

[15] Giacomo Mauro D'Ariano, Giulio Chiribella, and Paolo Perinotti. Quantum theory from first principles: an informational approach. Cambridge University Press, 2017. URL: https://doi.org/10.1017/ 9781107338340.

[16] Howard Barnum, Jonathan Barrett, Matthew Leifer, and Alexander Wilce. Teleportation in general probabilistic theories, 2008. arXiv:0805.3553.

[17] Howard Barnum and Alexander Wilce. Information processing in convex operational theories. Electronic Notes in Theoretical Computer Science, 270(1):3 15, 2011. Proceedings of the Joint 5th International Workshop on Quantum Physics and Logic and 4th Workshop on Developments in Computational Models (QPL/DCM 2008). URL: https://doi. org $/ 10.1016 / j$. entcs . 2011.01.002.

[18] Bob Coecke. Kindergarten quantum mechanics: Lecture notes. AIP Conference Proceedings, 810(1):81-98, 2006. URL: https: //doi.org/10.1063/1.2158713.

[19] Bob Coecke and Aleks Kissinger. Picturing Quantum Processes: A First Course in Quantum Theory and Diagrammatic Reasoning. Cambridge University Press, 2017. URL: https://doi.org/10.1017/ 9781316219317.

[20] Günther Ludwig. Foundations of quantum mechanics II. New York, NY (United States); Springer-Verlag, 1985. URL: https : //doi.org/10.1007/978-3-662-28726-2.

[21] Alexander Wilce. Test spaces and orthoalgebras. In Bob Coecke, David Moore, and Alexander Wilce, editors, Current Research in Operational Quantum Logic: Algebras, Categories, Languages, pages 81-114. Springer Netherlands, Dordrecht, 2000. URL: https://doi.org/10.1007/ 978-94-017-1201-9_4.

[22] Michael A. Nielsen and Isaac L. Chuang. Quantum Computation and Quantum Information: 10th Anniversary Edition. Cambridge University Press, 2010. URL: https: //doi.org/10.1017/CB09780511976667.

[23] Alessandro Bisio, Giacomo Mauro D'Ariano, and Alessandro Tosini. Dirac quantum cellular automaton in one dimension: Zitterbewegung and scattering from potential. Phys. Rev. A, 88:032301, Sep 2013. URL: https://doi.org/10.1103/ PhysRevA. 88.032301.

[24] Alessandro Bisio, Giacomo Mauro D'Ariano, and Alessandro Tosini. Quantum field as a quantum cellular automaton: The dirac free evolution in one dimension. Annals of Physics, 354:244 - 264, 2015. URL: https: //doi.org/10.1016/j . aop.2014.12.016.

[25] Giacomo Mauro D'Ariano and Paolo Perinotti. Derivation of the dirac equation from principles of information processing. Phys. Rev. A, 90:062106, Dec 2014. URL: 
https://doi.org/10.1103/PhysRevA.90. 062106.

[26] Alessandro Bisio, Giacomo Mauro D'Ariano, Paolo Perinotti, and Alessandro Tosini. Weyl, Dirac and Maxwell quantum cellular automata. Foundations of Physics, 45(10):1203-1221, Oct 2015. URL: https: //doi.org/10.1007/s10701-015-9927-0.

[27] Richard P. Feynman. Simulating physics with computers. International Journal of Theoretical Physics, 21(6):467-488, 1982. URL: https://doi.org/10.1007/ BF02650179.

[28] Wim van Dam. Quantum Cellular Automata. PhD thesis, University of Nijmegen, 1996. URL: https://sites.cs.ucsb.edu/ $\sim$ vandam/qca.pdf.

[29] J. Watrous. On one-dimensional quantum cellular automata. In Proceedings of IEEE 36th Annual Foundations of Computer Science, pages 528-537, Oct 1995. URL: https: //doi.org/10.1109/SFCS.1995.492583.

[30] J Gruska. Quantum Computing. McGrawHill, 1999.

[31] B. Schumacher and R. F. Werner. Reversible quantum cellular automata, 2004. arXiv: quant-ph/0405174.

[32] Pablo Arrighi, Vincent Nesme, and Reinhard Werner. Unitarity plus causality implies localizability. Journal of Computer and System Sciences, 77(2):372 - 378, 2011. Adaptivity in Heterogeneous Environments. URL: https://doi.org/10.1016/j.jcss. 2010.05 .004 .

[33] D. Gross, V. Nesme, H. Vogts, and R. F. Werner. Index theory of one dimensional quantum walks and cellular automata. Communications in Mathematical Physics, 310(2):419-454, Mar 2012. URL: https: //doi.org/10.1007/s00220-012-1423-1.

[34] Pablo Arrighi, Renan Fargetton, Vincent Nesme, and Eric Thierry. Applying causality principles to the axiomatization of probabilistic cellular automata. In Benedikt Löwe, Dag Normann, Ivan Soskov, and Alexandra Soskova, editors, Models of Computation in Context, pages 1-10, Berlin,
Heidelberg, 2011. Springer Berlin Heidelberg. URL: https://doi.org/10.1007/ 978-3-642-21875-0_1.

[35] Sergey B. Bravyi and Alexei Yu. Kitaev. Fermionic quantum computation. Annals of Physics, 298(1):210 - 226, 2002. URL: https://doi.org/10.1006/ aphy. 2002.6254 .

[36] G. M. D'Ariano, F. Manessi, P. Perinotti, and A. Tosini. Fermionic computation is non-local tomographic and violates monogamy of entanglement. EPL (Europhysics Letters), 107(2):20009, jul 2014. URL: https://doi.org/10.1209/ 0295-5075/107/20009.

[37] Giacomo Mauro D'Ariano, Franco Manessi, Paolo Perinotti, and Alessandro Tosini. The feynman problem and fermionic entanglement: Fermionic theory versus qubit theory. International Journal of Modern Physics A, 29(17):1430025, 2014. URL: https://doi. org/10.1142/S0217751X14300257.

[38] Y. Aharonov, L. Davidovich, and N. Zagury. Quantum random walks. Phys. Rev. A, 48:1687-1690, Aug 1993. URL: https: //doi.org/10.1103/PhysRevA.48.1687.

[39] Dorit Aharonov, Andris Ambainis, Julia Kempe, and Umesh Vazirani. Quantum walks on graphs. In Proceedings of the Thirty-Third Annual ACM Symposium on Theory of Computing, STOC '01, pages 5059, New York, NY, USA, 2001. Association for Computing Machinery. URL: https: //doi.org/10.1145/380752.380758.

[40] Peter L. Knight, Eugenio Roldán, and J. E. Sipe. Propagating quantum walks: The origin of interference structures. Journal of Modern Optics, 51(12):1761-1777, 2004. URL: https://doi.org/10.1080/ 09500340408232489.

[41] Norio Konno. A new type of limit theorems for the one-dimensional quantum random walk. J. Math. Soc. Japan, 57(4):11791195, 10 2005. URL: https://doi.org/10. 2969/jmsj/1150287309.

[42] Hilary A Carteret, Mourad E H Ismail, and Bruce Richmond. Three routes to the exact asymptotics for the one-dimensional quantum walk. Journal of Physics A: Mathe- 
matical and General, 36(33):8775-8795, aug 2003. URL: https://doi.org/10.1088/ 0305-4470/36/33/305.

[43] Andris Ambainis, Eric Bach, Ashwin Nayak, Ashvin Vishwanath, and John Watrous. One-dimensional quantum walks. In Proceedings of the Thirty-Third Annual ACM Symposium on Theory of Computing, STOC '01, pages 37-49, New York, NY, USA, 2001. Association for Computing Machinery. URL: https://doi .org/10.1145/380752. 380757.

[44] Alessandro Bisio, Giacomo Mauro D'Ariano, Paolo Perinotti, and Alessandro Tosini. Thirring quantum cellular automaton. Phys. Rev. A, 97:032132, Mar 2018. URL: https://doi.org/10.1103/PhysRevA.97. 032132.

[45] Paolo Perinotti and Leopoldo Poggiali. Scalar fermionic cellular automata on finite cayley graphs. Phys. Rev. A, 98:052337, Nov 2018. URL: https://doi.org/10.1103/ PhysRevA.98.052337.

[46] Giacomo Mauro D'Ariano and Paolo Perinotti. Quantum cellular automata and free quantum field theory. Frontiers of Physics, 12(1):120301, Sep 2016. URL: https://doi.org/10.1007/ s11467-016-0616-z.

[47] P. de la Harpe. Topics in Geometric Group Theory. Chicago Lectures in Mathematics. University of Chicago Press, 2000. URL: https://press.uchicago.edu/ucp/ books/book/chicago/T/bo3641370.html.

[48] Shuichi Inokuchi and Yoshihiro Mizoguchi. Generalized partitioned quantum cellular automata and quantization of classical ca. Int. Journ. of Unconventional Computing, 1:149-160, 2005. arXiv:quant-ph/ 0312102.

[49] J. von Neumann. On infinite direct products. Compositio Mathematica, 6:177, 1939. URL: http://www. numdam.org/ item/CM_1939__6_-1_0.

[50] F. J. Murray and J. v. Neumann. On rings of operators. Annals of Mathematics, 37(1):116-229, 1936. URL: https://doi. org/10.2307/1968693.
[51] Rainer J. Nagel. Order unit and base norm spaces. In A. Hartkämper and H. Neumann, editors, Foundations of Quantum Mechanics and Ordered Linear Spaces: Advanced Study Institute Marburg 1973, pages 23-29. Springer Berlin Heidelberg, Berlin, Heidelberg, 1974. URL: https://doi.org/10. 1007/3-540-06725-6_4.

[52] David Beckman, Daniel Gottesman, M. A. Nielsen, and John Preskill. Causal and localizable quantum operations. Phys. Rev. A, 64:052309, Oct 2001. URL: https://doi. org/10.1103/PhysRevA .64.052309.

[53] T Eggeling, D Schlingemann, and R. F Werner. Semicausal operations are semilocalizable. Europhysics Letters (EPL), 57(6):782-788, mar 2002. URL: https:// doi.org/10.1209/epl/i2002-00579-4.

[54] Benjamin Schumacher and Michael D. Westmoreland. Locality and information transfer in quantum operations. Quantum Information Processing, 4(1):13-34, February 2005. URL: https://doi.org/10.1007/ s11128-004-3193-y.

[55] Jonathan Barrett, Robin Lorenz, and Ognyan Oreshkov. Quantum causal models. 2019. arXiv: 1906.10726.

[56] Pablo Arrighi, Nicolas Schabanel, and Guillaume Theyssier. Stochastic cellular automata: Correlations, decidability and simulations. Fundamenta Informaticae, 126:121156, 2013. URL: https://doi.org/10. 3233/FI-2013-875.

[57] Giacomo Mauro D'Ariano, Marco Erba, and Paolo Perinotti. Isotropic quantum walks on lattices and the weyl equation. Phys. Rev. A, 96:062101, Dec 2017. URL: https://doi. org/10.1103/PhysRevA.96.062101.

[58] Lucien Hardy and William K. Wootters. Limited holism and real-vector-space quantum theory. Foundations of Physics, 42(3):454-473, 2012. URL: https://doi . org/10.1007/s10701-011-9616-6.

[59] Karl-Peter Hadeler and Johannes Müller. Introduction. In Cellular Automata: Analysis and Applications, pages 1-17. Springer International Publishing, Cham, 2017. URL: https://doi.org/10.1007/ 978-3-319-53043-7_1. 
[60] Pablo Arrighi and Vincent Nesme. The block neighborhood. In Jarkko Kari, editor, Second Symposium on Cellular Automata "Journeés Automates Cellulaires", JAC 2010, Turku, Finland, December 15-17, 2010. Proceedings, pages 43-53. Turku Center for Computer Science, 2010. URL: https://hal archives-ouvertes. fr/hal-00542488.

[61] Pablo Arrighi, Vincent Nesme, and Reinhard F. Werner. Bounds on the speedup in quantum signaling. Phys. Rev. A, 95:012331, Jan 2017. URL: https://doi.org/10. 1103/PhysRevA. 95.012331.

[62] Michael Freedman and Matthew B. Hastings. Classification of quantum cellular automata. Communications in Mathematical Physics, 376(2):1171-1222, 2020. URL: https://doi.org/10.1007/ s00220-020-03735-y.

[63] Michael Freedman, Jeongwan Haah, and Matthew B Hastings. The group structure of quantum cellular automata. 2019. arXiv: 1910.07998.

[64] Terry Farrelly. A review of quantum cellular automata, 2019. arXiv:1904.13318.

[65] P. Arrighi. An overview of quantum cellular automata. Natural Computing, 18(4):885899, 2019. URL: https://doi.org/10. 1007/s11047-019-09762-6.

[66] Alessandro Bisio, Giacomo D'Ariano, Nicola Mosco, Paolo Perinotti, and Alessandro Tosini. Solutions of a two-particle interacting quantum walk. Entropy, 20(6):435, Jun 2018. URL: https://doi.org/10.3390/ e20060435. 